\title{
Dissecting and Modeling Oncogene Dependent Molecular Mechanisms in Lymphoma Genesis and Progression
}

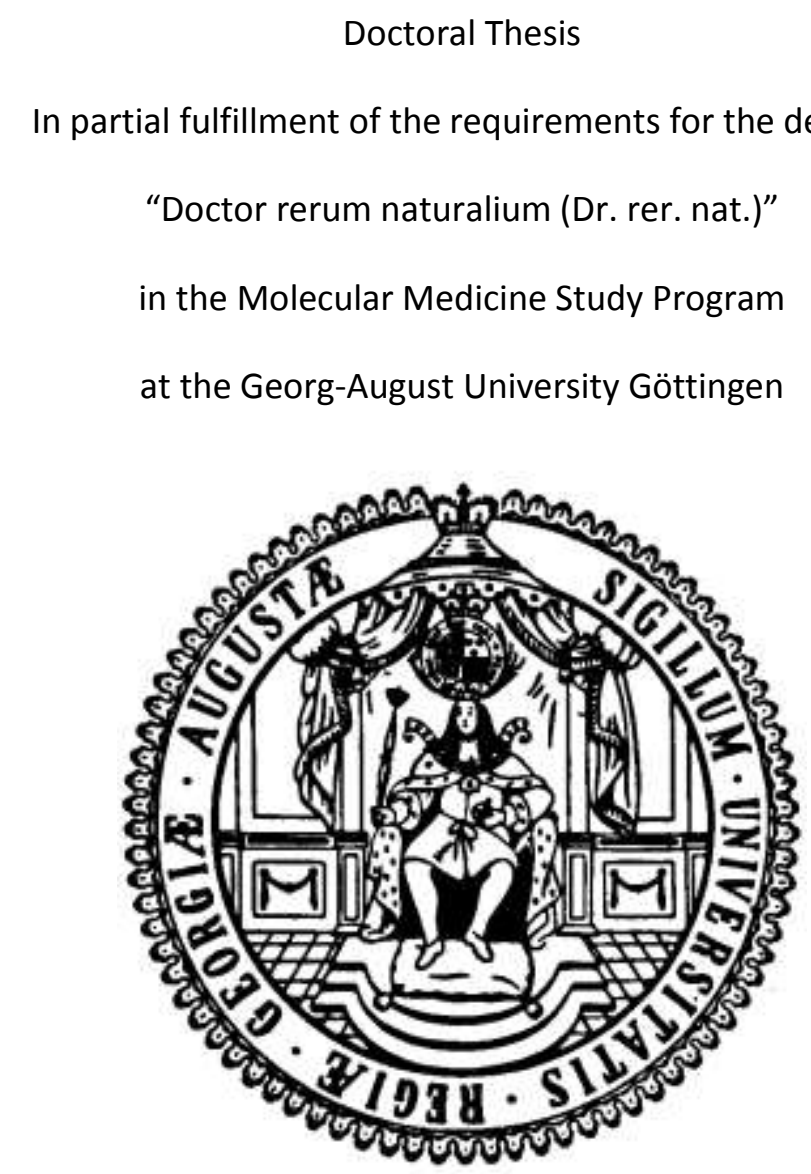

submitted by Elisabeth Hand

born in Berlin 



\section{Thesis Committee}

Prof Dr. Dieter Kube (Supervisor)

E-Mail

dkube@med.uni-goettingen.de

Phone

0049-551-391537

Postal Address

Universitätsmedizin Göttingen

Zentrum Innere Medizin

Abteilung Hämatologie und Onkologie

Robert-Koch-Straße 40

37075 Göttingen

Prof Dr. Mikael Simons

E-Mail

msimons@gwdg.de

Phone

0049-551-3899533

Postal Address

Max-Planck-Institut für experimentelle Medizin

Hermann-Rein-Str. 3

37075 Göttingen

Prof Dr. Peter Burfeind

E-Mail

pburfei@gwdg.de

Phone

0049-551-397595

Postal Address

Universitätsmedizin Göttingen

Zentrum Hygiene und Humangenetik

Institut für Humangenetik

Heinrich-Düker-Weg 12

37073 Göttingen

Date of Disputation: 


\section{Affidavit}

By this I declare that I independently authored the presented thesis:

"Dissecting and modeling oncogene dependent molecular mechanisms in lymphoma genesis and progression"

and that I did not use other auxiliary means than indicated. Paragraphs that are taken from other publications, by wording or by sense, are marked in every case with a specification of the literary source.

Furthermore I declare that I carried out the scientific experiments following the principles of Good Scientific Practice according to the valid "Richtlinien der Georg-August-Universität Göttingen zur Sicherung guter wissenschaftlicher Praxis".

Elisabeth Hand

Göttingen, August 2013 


\section{Table of Contents}

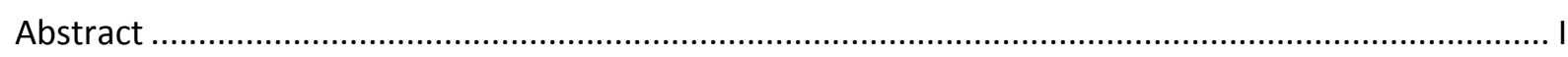

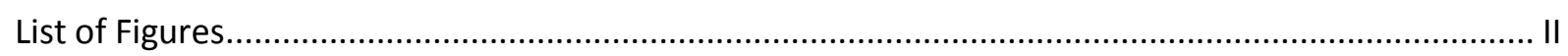

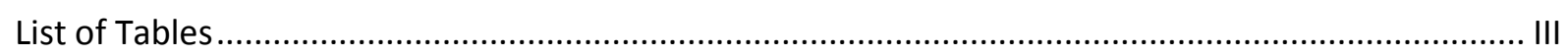

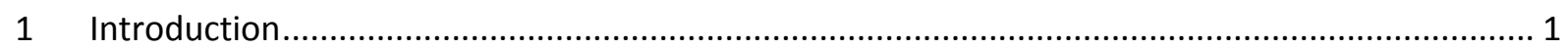

1.1 Development and transformation of germinal center B cells in lymph nodes ................... 1

1.2 Paracrine and autocrine signaling influence B cell development and transformation .......... 4

1.2.1 B cell receptor activated signaling and its implications in lymphomagenesis ..............4

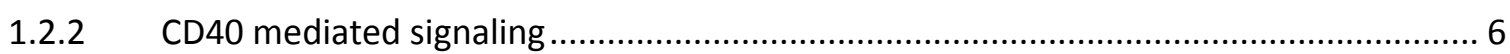

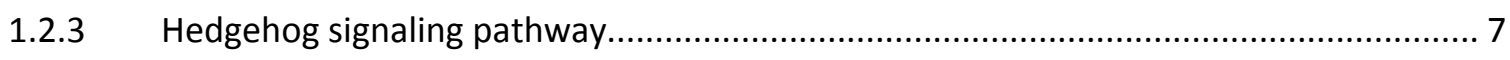

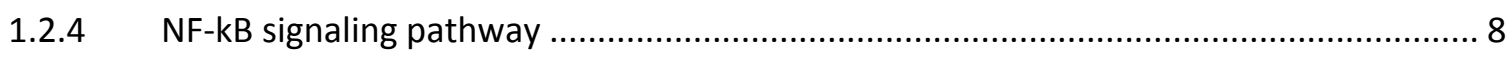

1.2.5 Mitogen activated protein kinase (MAPK) signaling pathways................................ 9

1.3 Strategies to describe and stratify lymphoma cases based on gene expression profiling.... 11

1.3.1 Diffuse large B cell lymphoma (DLBCL) ........................................................ 11

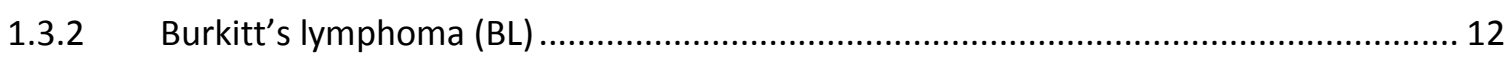

1.3.3 Surrogate markers for oncogenic pathway activation ......................................... 14

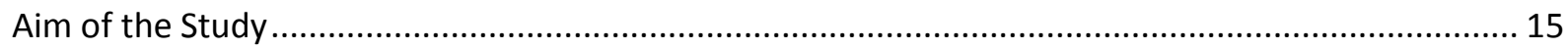

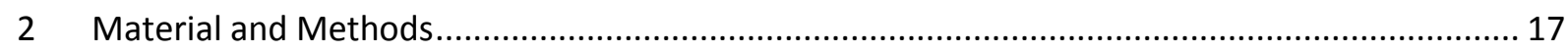

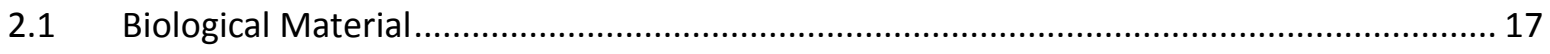

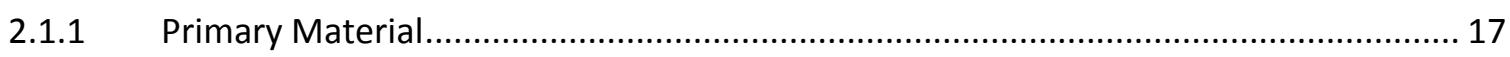

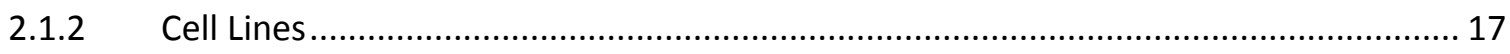

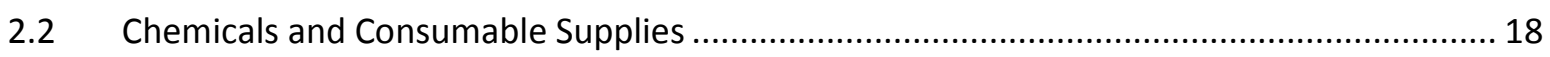

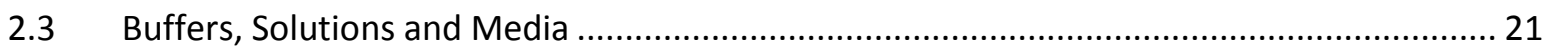

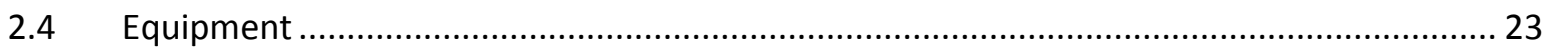

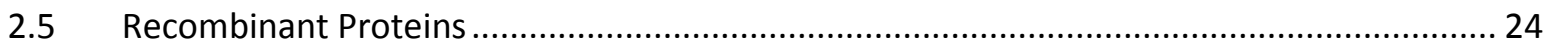

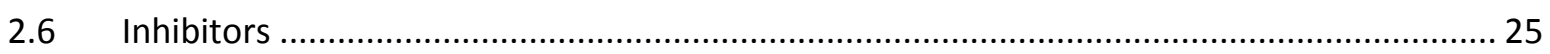

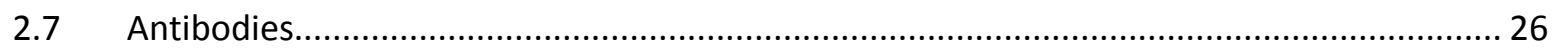

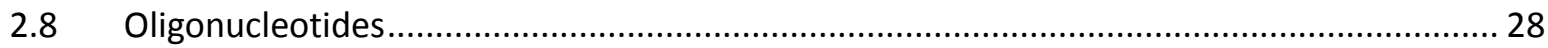




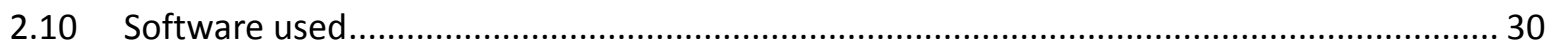

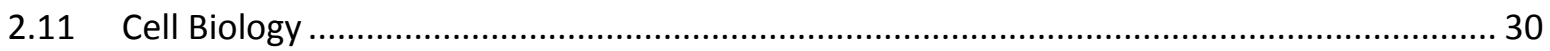

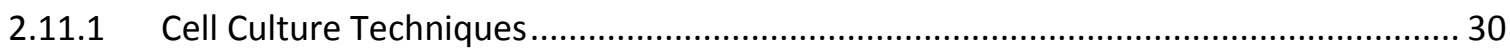

2.11.2 Activation of B cells with Soluble Stimulating Factors ........................................ 30

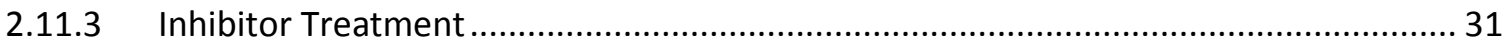

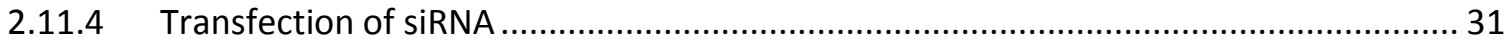

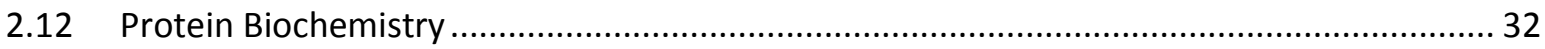

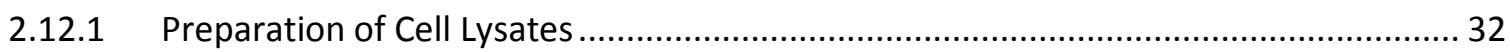

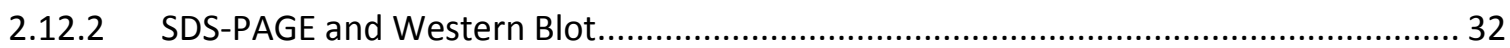

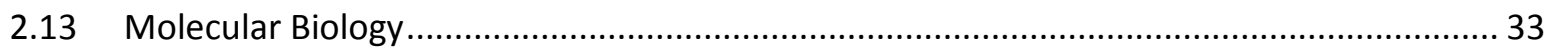

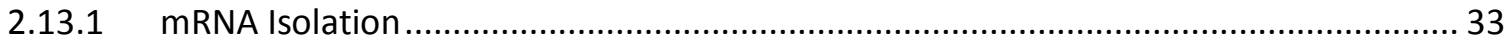

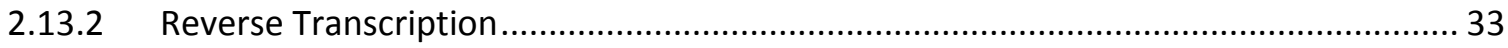

2.13.3 qRT-PCR (quantitative Real Time - Polymerase Chain Reaction).............................. 33

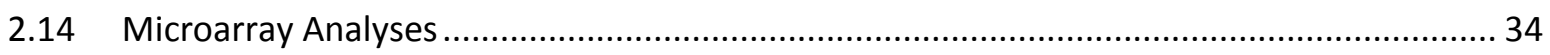

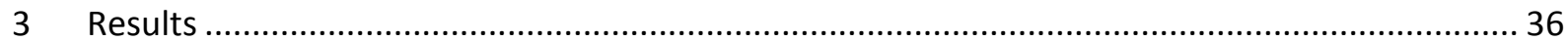

3.1 Temporal pattern of whole genome gene expression changes upon signaling pathway activation 36

3.1.1 $\alpha$-IgM stimulation of BL cells changes the $\mathrm{mBL}$ index towards non-mBLs................38

3.1.2 Causality Network of BCR induced gene expression correlations........................... 39

3.1.3 A mathematically new method to analyze time courses identifies differentially expressed clusters of genes.

3.2 Ptch1 and Hedgehog signaling in lymphoma ..................................................... 49

3.2.1 Expression profile of Hedgehog signaling components ..........................................50

3.2.2 Lymphoma cell lines neither secrete nor respond to Sonic hedgehog .....................53

3.2.3 PI3K-mediated regulation of PTCH1, c-MYC and LEF1 mRNA expression.................. 55

3.3 Analyzing intersections in signaling pathways via use of specific chemical inhibitors ........ 58

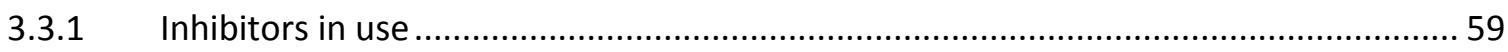

3.3.2 Differential gene expression on the whole genome level upon inhibitor use .............63 
3.3.3 Nested Effects Models propose a controversial role of Tak1 in CD40 and BCR mediated signaling. 66

3.3.4 NF-kB signaling upon BCR crosslink in Burkitt's lymphoma ....................................... 73

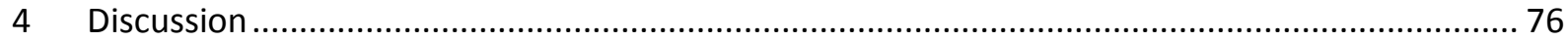

4.1 Analyzing the temporal pattern of gene expression changes ........................................... 76

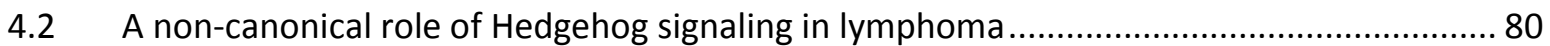

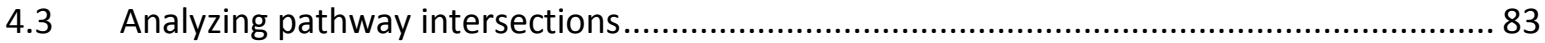

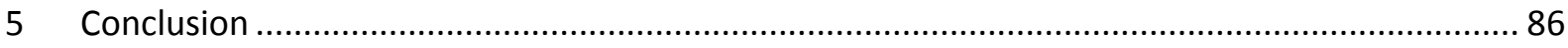

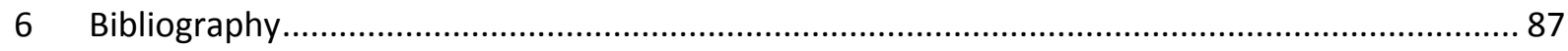

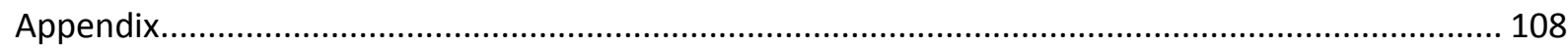

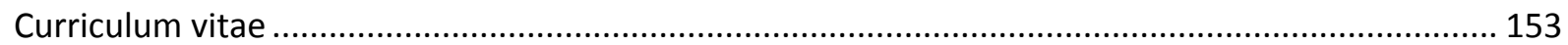

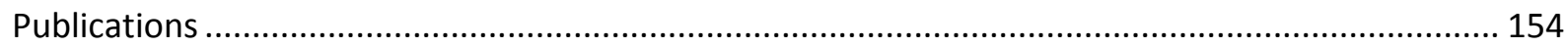

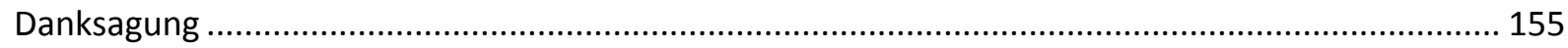




\begin{abstract}
Aggressive Non-Hodgkin lymphomas (aNHL) are a heterogeneous group of lymphomas. The constellation of oncogenic pathway activities can vary in patients with the same malignant disease. The pathological mechanisms behind the current criteria to distinguish individual aNHL subtypes are still poorly understood. Molecular models depicting the dynamics of oncogenic signaling and cross talk of the pathways can help to obtain a better understanding of lymphoma pathogenesis. Thereby the development of future targeted therapies, that will have to intervene with specific signaling modules to reverse pathway deregulation, will be enabled. We postulate that the description of a time-resolved global gene expression change provides new insights into the dynamics of oncogenic signaling and that the intervention into pathway intersections allows identifying new essential pathway nodes and cross talks for targeted therapies.
\end{abstract}

To gain closer insight into distinct cell signaling networks and their relevance for NHL subtypes, firstly, high-dimensional data of time-resolved gene expression changes in BL2 cells induced by BCR or CD40 activation were generated. By using a newly established computational method we were able to describe 20 disjoint sets of genes to group their time courses. 15 significant sets of genes were identified for BCR stimulated samples and 5 significant sets of genes for CD40 activated samples, all displaying distinct time courses. We discovered a co-repression of LEF1, PTCH1 and NOTCH1 by activation of the B cell receptor. Functional assays revealed that BL cells are not responsive to Sonic hedgehog stimulation, but evidence is provided for a c-Myc-driven non-canonical regulation of this pathway. Furthermore, a working model for a causality network of BCR induced gene expression correlations was compiled. In addition, sustained activated BCR signaling induced a change of the gene expression profile towards a certain 'DLBCL-likeness'. Therefore, a first step to develop a model displaying the causative factors for the non-mBL signature is provided. Secondly, the gene expression changes of BCR or CD4OL stimulated BL2 cells after chemical inhibition of Tak1, JNK, p38 and IKK2 were monitored on the whole genome level. In BCR stimulated cells the activity of PI3K and Mek1/2 was as well inhibited. Additional biochemical analyses supported the view that Tak1 is a pivotal modulator of both CD40 and BCR mediated p38 and NF-KB signaling. Moreover, using (5Z)-7-Oxozeanol as well as TAK1-directed siRNA to inhibit Tak1 activity, a corresponding Tak1Erk1/2 subnetwork was described.

Thus, the present study and the herein allocated data provide deep insight into oncogenic pathway activities and enable a large variety of continuing studies, which may help to obtain a better understanding of the biology of lymphoma. 


\section{List of Figures}

Figure 1-1 Simplified scheme of B cell differentiation in the germinal center. ..................................... 2

Figure 1-2 Simplified scheme of the basics of B cell receptor signaling. …........................................... 5

Figure 3-1 Time course analyses of gene expression changes on the whole genome level upon stimulation of human transformed germinal center B cells.

Figure 3-2 Sustained BCR activation in BL cells shifts $\mathrm{mBL}$ index towards the index of non-mBLs. ..... 39

Figure 3-3 Causality network displaying anti-IgM stimulated gene expression correlations. 40

Figure 3-4 Comparison of time courses of genes commonly regulated through BCR and CD40......

Figure 3-5 Distinct time courses for anti-IgM and CD40 stimulated BL2 cells can be determined by testing for stationarity

Figure 3-6 MYC, PTCH1 and LEF1 mRNA expression is suppressed in BL2 cells upon anti-IgM stimulation

Figure 3-7 Differential expression and induction of Hh signaling components in Burkitt's and diffuse large B cell lymphoma cell lines through anti-IgM stimulation....

Figure 3-8 Lymphoma cell lines do not produce Shh, neither do they respond to Shh nor to cyclopamine.

Figure 3-9 Regulation of PTCH1, MYC and LEF1 is mediated through PI3K-PLCY-PKC signaling.......... 56

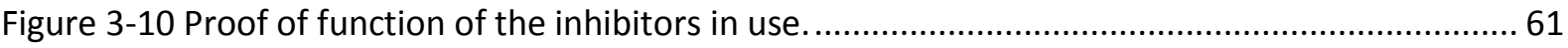

Figure 3-11 Effects of inhibitors used in microarray analyses.

Figure 3-12 Role of Tak1 in CD40 and BCR mediated signaling proposed by a first generation Nested Effects Model.

Figure 3-13 Tak1 presents as major signal modulator in CD40 and BCR mediated signaling.

Figure 3-14 Comparison of two Burkitt's lymphoma cell lines with regard to signaling interruption upon Tak1 inhibition

Figure 3-15 Differences in NF-kB activatability in Burkitt's lymphoma and Diffuse large B cell lymphoma.

Figure A-1 Bayesian Hierarchical Clustering reveals 27 clusters of genes bearing differential expression over time. 


\section{List of Tables}

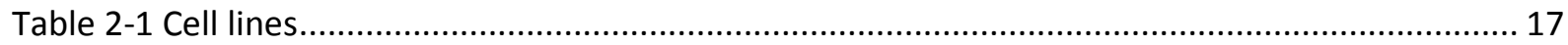

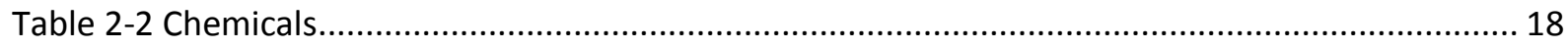

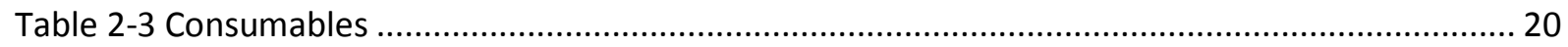

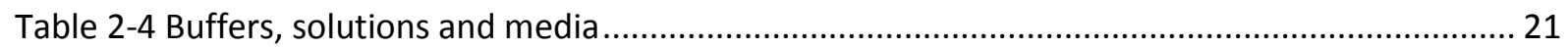

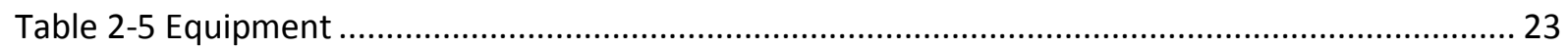

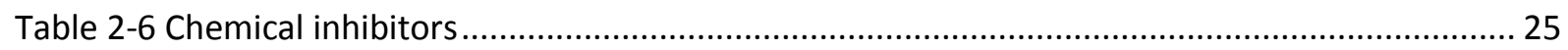

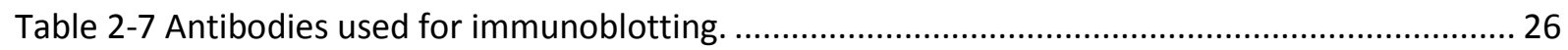

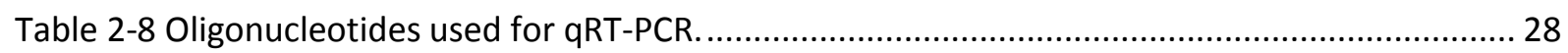

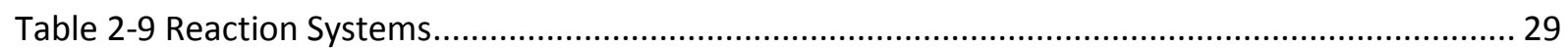

Table 2-10 Master Mix and thermocycler program for reverse transcription of mRNA ..................... 33

Table 3-1 Differential expression in human transformed germinal center B cells in response to

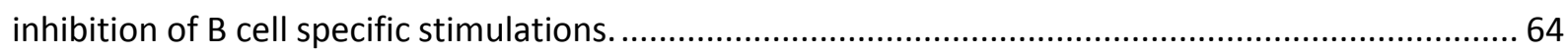

Table A-1 Clusters of genes identified upon BCR crosslinked BL2 cells (BHC, SIMoNe). ................... 108

Table A-2 Gene set enrichment analysis for manually picked clusters ( $\mathrm{cl} 6, \mathrm{cl} 8$ and $\mathrm{cl} 11)$............... 129

Table A-3 List of genes, taken for comparison of CD40 and BCR stimulated BL cells by testing for stationarity.

Table A-4 Testing for stationarity reveals 5 distinct and disjoint groups of genes, changing their expression after SCD40L stimulation.

Table A-5 Testing for stationarity reveals 15 distinct and disjoint groups of genes in BCR-resolved time series analysis. 
Introduction

\section{Introduction}

Approved living circumstances in the Western World as well as the demographic change and prolonged life expectancies have favored cancer becoming a leading cause of mortality worldwide. According to the World Health Organization (WHO), cancer accounted for $13 \%$ of all deaths in Europe in 2008 (Ferlay et al., 2010). Non-Hodgkin lymphoma (NHL) is estimated to be the tenth most common cancer worldwide, with an increasing incidence over the last decades and highest rates being reported in the most economically developed countries (Cancer Research UK, 2010). NHLs are a subgroup of lymphoma, which comprise a heterogeneous group of hematological malignancies that originate mainly from B cells (95\%) and usually form solid tumors. NHL can be divided into indolent (slow-growing) and aggressive (fast-growing) subtypes (Jaffe et al., 1998). B cell aggressive NHL $(\mathrm{aNHL})$ is a very heterogeneous group of malignancies. In the last decade, clinical and experimental investigations complemented by novel molecular genome-wide investigations like gene expression profiling and whole-genome sequencing have helped to expand our understanding of the biology and diversity of different types of aNHL.

\subsection{Development and transformation of germinal center B cells in lymph nodes}

In the past 20 years, exciting progress has been made to elucidate the cellular origin of $\mathrm{B}$ cell lymphomas as well as the key events leading to transformation. Furthermore, large efforts were made to characterize the pathogenesis of lymphoma (reviewed in Klein and Dalla-Favera, 2008; Küppers, 2005). The development of B cells as part of the adaptive immune response is strictly dependent on their surrounding microenvironment. To achieve optimal antigen recognition by their surface antigen receptor, B cells undergo several maturation, differentiation and proliferation steps in different compartments of the body. Early B cell development occurs in the bone marrow, consists of rearrangement of the heavy and light chain immunoglobulin (Ig) genes and concludes with a B cell carrying a functional, non-autoreactive $B$ cell receptor (BCR). Those cells differentiate into mature naïve B cells, leave the bone marrow and circulate in the blood and lymphatic system to performe the role of immune surveillance. In contrast, precursor B cells, which were unable to maturate a functionally active BCR, undergo apoptosis.

Upon antigen binding to the $B C R$, mature naïve $B$ cells can be activated. In the T-cell-dependent response to exogenous antigens, proliferating B cells form so called 'germinal centers' (GCs) in the follicles of peripheral lymphoid tissues including lymph nodes. In the early 1990 it was shown that GCs are the main site where the immunoglobulins are modified and finalized through somatic hypermutation (SHM) and class-switch recombination (CSR) (Berek et al., 1991; Jacob et al., 1991). 
Today, it is broadly accepted, that the GC microenvironment is the general source of memory B cells and plasma cells (Figure 1-1), producing high-affinity antigen receptors and therefore being essential for an effective protection against invading microorganisms (MacLennan, 1994). In addition, the GC micromilieu seems to play an important role in lymphoma pathogenesis, since several studies observed that most types of malignant B cells forming B cell lymphomas reflect their origin by being 'frozen' at that particular differentiation status. In fact, nearly all B cell aNHLs display somatically mutated IgV genes, indicating that they are derived from GC or post-GC B cells (Küppers et al., 1999; Stevenson et al., 2001).

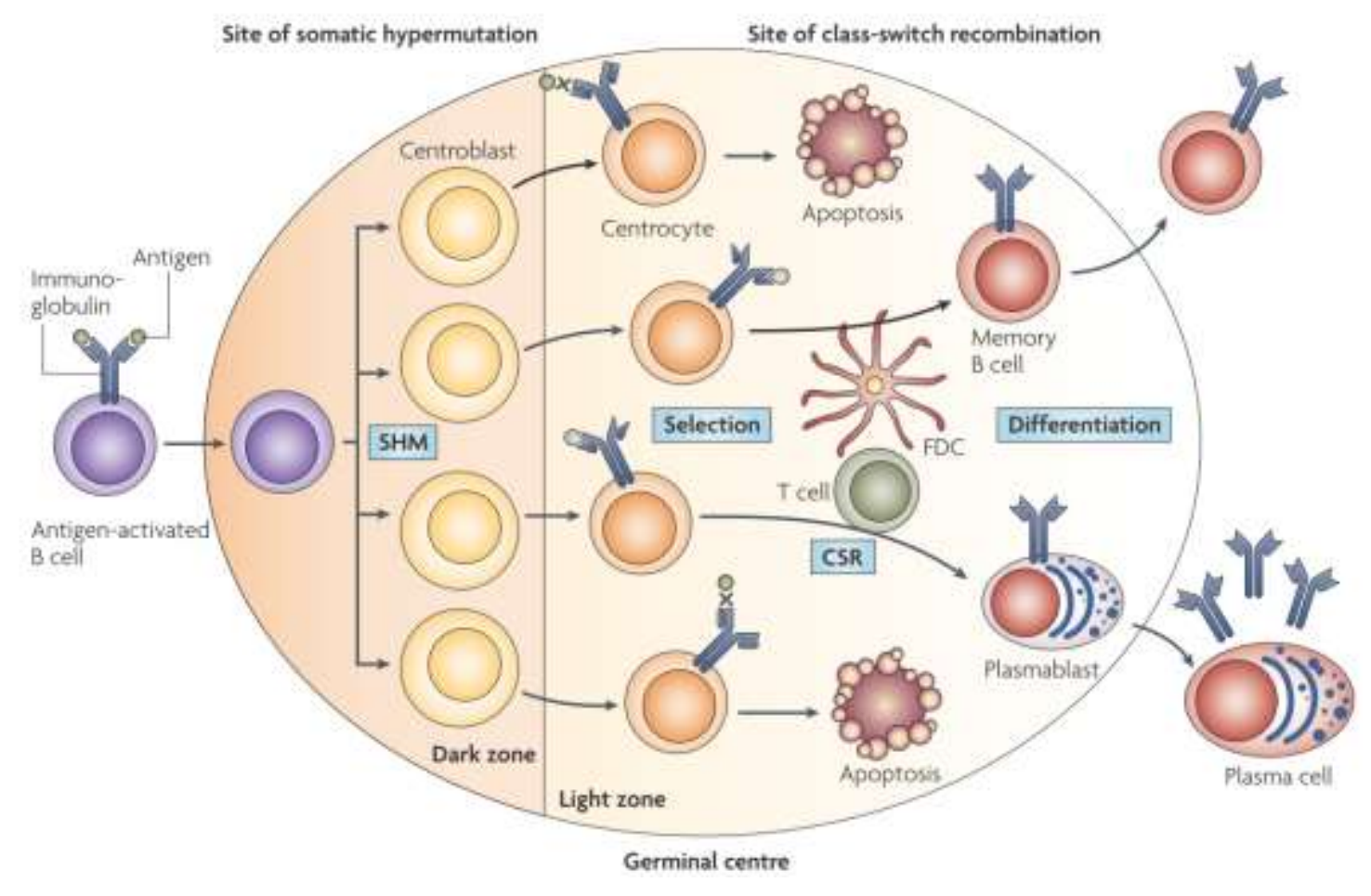

Figure 1-1 Simplified scheme of B cell differentiation in the germinal center. Antigen-activated B cells enter peripheral lymphoid organs and form a germinal center (GC). GCs are polarized into dark and light zones. The dark zone mainly consists of proliferating GC B cells, in which somatic hypermutation (SHM) occurs. By this a high rate of mutations in the variable (V)-region of Ig genes are introduced. GC B cells with mutated BCRs can then enter the light zone (now mainly consisting of resting cells) and are positively selected for high affinity antigen receptors. GC B cells exhibiting a BCR with lower affinity for the antigen undergo apoptosis. The selection process is presumably controlled through the close contact to $T$ cells and follicular dendritic cells (FDCs). A fraction of the positively selected cells encounter class-switch recombination (CSR). Finally, GC B cells differentiate into plasma cells or memory $B$ cells and leave the germinal center microenvironment into the periphery. Figure taken from (Klein and Dalla-Favera, 2008).

Indeed, both of the germinal center-intrinsic processes somatic hypermutation and class-switch recombination may lead to genetic aberrations promoting lymphomagenesis (reviewed in Küppers et al., 1999). Thus, mistakes or malfunctions in the immunoglobulin gene remodeling mechanisms chromosomal translocations and aberrant SHM - can be found as the main causes for genetic lesions in B cell NHLs (reviewed by Küppers and Dalla-Favera, 2001). A typical functional consequence is the 
deregulated expression of proto-oncogenes, because regulatory sequences have been juxtaposed in close proximity to the intact coding domain of the target gene from heterologous chromosomes by translocations.

Thus, chromosomal translocations, which involve for example $B C L-6$ (B cell lymphoma 6 ), result in prevention of gene silencing at the end of the GC reaction and can be frequently found in diffuse large B cell lymphoma (DLBCL) (see chapter 1.3.1 for more detail). Enhanced expression of the protooncogene $c-M Y C$ results from the translocation into the immunoglobulin heavy chain or light chain loci and is associated with $100 \%$ of Burkitt's lymphoma cases (see chapter 1.3.2 for more details on Burkitt's lymphoma) and up to $10 \%$ of DLBCL cases (Klein and Dalla-Favera, 2008). Moreover, additional genetic alterations occur in the different subtypes of aNHL, altogether leading to deregulated signaling events promoting oncogenic transformation. 
Introduction

\subsection{Paracrine and autocrine signaling influence B cell development and} transformation

Proliferation, differentiation and survival of activated B cells in secondary lymphatic organs rely tremendously on paracrine signals mediated by the microenvironment. This is provided in the GC, for example by $T$ cells and follicular dendritic cells (FDCs). These bystander cells supply the maturating $B$ cells with important survival signals, as for example CD40L expressed by CD4 ${ }^{+} \mathrm{T}$ cells (see chapter 1.2.2 for further details) or Sonic hedgehog (Shh) expressed by FDCs in the GC (see chapter 1.2.3 for more information). On the other hand, autocrine signaling that is not dependent on surrounding microenvironmental cells determines the $B$ cell fate in a similar manner. One central player includes the $B$ cell receptor. Normal B cells depend on BCR expression for survival and undergo stringent selection for expression of the appropriate BCR throughout their lives. Moreover, the selection for expression of a functional BCR also seems to occur in most aNHL subtypes (Gunvén et al., 1980; Segal et al., 1991; Yano et al., 1992). Additionally, deregulations of BCR signaling or signaling mediated through BCR or CD40 activation seem to operate in BL and DLBCL (chapter 1.2.1 and 1.2.2). In the following paragraphs a brief overview will be given of BCR, CD40 and Hedgehog activated signaling pathways and the distinct mechanism involved in the respective pathway activations. As NF-KB (nuclear factor of kappa light polypeptide enhancer in B cells) and MAPK (mitogen-activated protein kinase) signaling are central to most of the here described signaling pathways, they will be described in more detail.

\subsubsection{B cell receptor activated signaling and its implications in lymphomagenesis}

Every normal $B$ cell and consequently every lymphoma has a unique $B C R$, whose signaling results in activation of the NF-KB, phosphoinositol 3-kinase (PI3K), MAPK, nuclear factor of activated T cells (NFAT) and RAS pathways, which foster proliferation and survival of normal and malignant B cells (Dal Porto et al., 2004). The BCR is a multimeric complex, consisting of an antigen-recognition structure and a membrane-bound immunoglobulin ( $(\mathrm{g})$, which itself lacks signaling capacity. Therefore, the BCR is non-covalently associated with CD79A $(\operatorname{Ig} \alpha)$ and $C D 79 B(\operatorname{Ig} \beta)$, each bearing an immunoreceptor tyrosine-based activation motif (ITAM) (Cambier, 1995; Reth, 1989), which transduces signals intracellularly (Figure 1-2). Antigen induced aggregation of the BCR induces ITAM phosphorylation by SRC-family kinases, which in turn leads to recruitment and activation of the tyrosine kinase SYK (Rowley et al., 1995; Saijo et al., 2003). The hereupon nucleated multiprotein complex consisting of various other kinases and adaptor proteins activates downstream kinases like Bruton's tyrosin kinase (Btk) and phospholipase C $\gamma 2$ (PLCY2) (Takata and Kurosaki, 1996). As consequence numerous signal transducers and second messengers are activated. Thus, for example PLCY2 triggers the release of calcium from the endoplasmatic reticulum thereby activating 
calcineurin and the transcription factor NFAT (Antony et al., 2004). Additionally, the increased calcium levels activate protein kinase $C \beta$ (PKC $\beta$ ), which in turn phosphorylates many substrates, including caspase recruitment domain-containing protein 11 (CARD11), a key signaling adaptor mediating NF-KB activation (Shinohara et al., 2005).

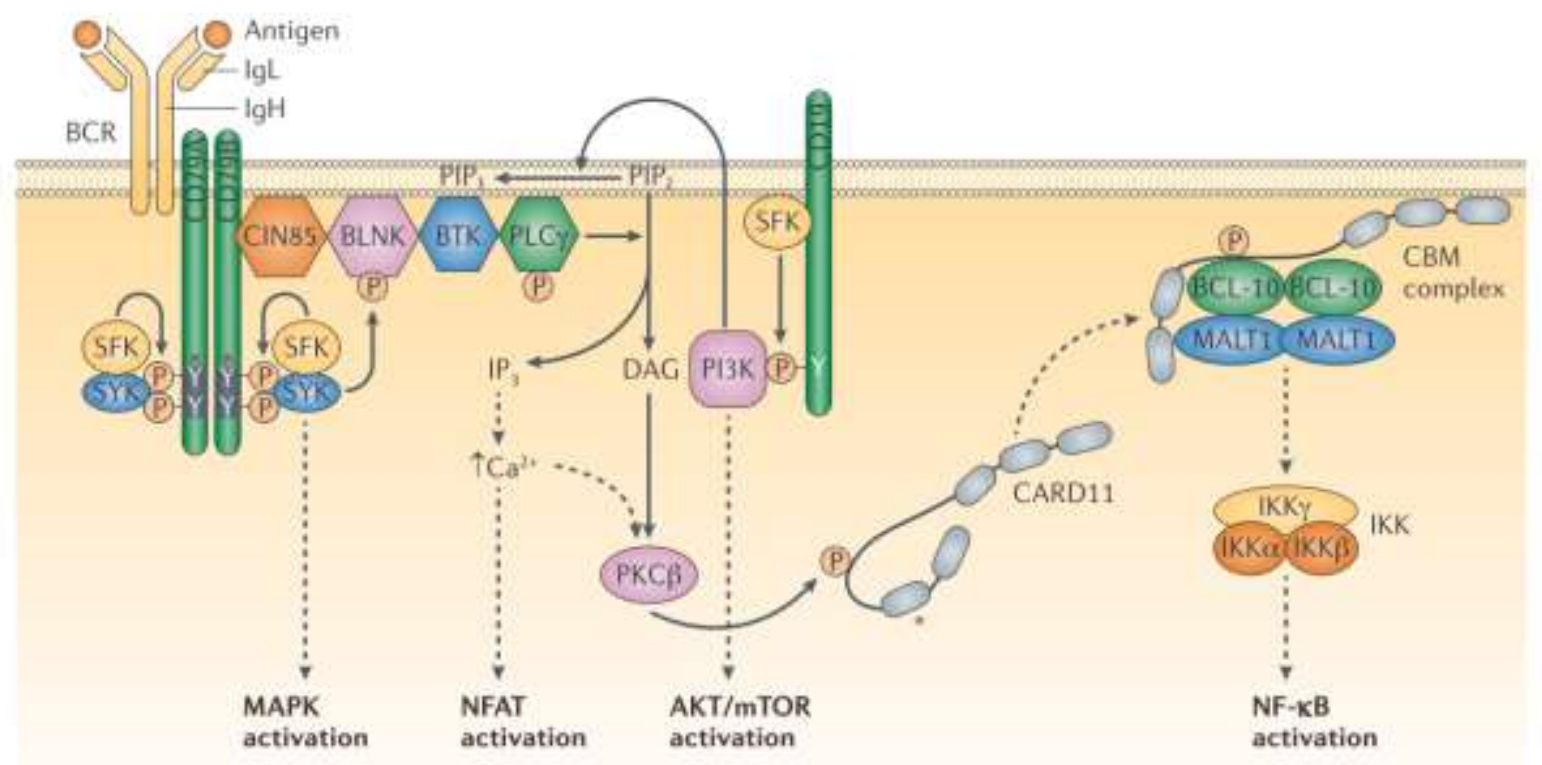

Figure 1-2 Simplified scheme of the basics of B cell receptor signaling. The scheme depicts the BCR, consisting of pairs of immunoglobulin heavy $(\mathrm{IgH})$ and light $(\mathrm{IgL})$ chains, whose variable region allows the BCR to bind to diverse antigens. The antibody portion of the BCR is coupled non-covalently with CD79A and CD79B, which mediate plasma membrane expression, signal transduction and receptor internalization. The ITAM region, containing two tyrosine residues $(\mathrm{Y})$ is phosphorylated by SRC-family kinases (SFK) upon antigen-induced aggregation of the BCR following SYK recruitment. Several signaling mediators are engaged and lead to the activation of various downstream signaling pathways, as indicated (refer to main text for details). CIN85, Cblinteracting protein of 85 kDa; BLNK, B cell linker protein; BTK, Bruton tyrosine kinase; PLC $\gamma$, phospholipase C $\gamma$; PIP2, phosphatidylinositol-4,5-biphosphate; PIP3, phosphatidylinositol-3,4,5-triphosphate; IP, inositol triphosphate; DAG, diacylglycerol; $P K C \beta$, protein kinase $C \beta$; PI3K, phosphoinositol 3-kinase; CARD11, caspase recruitment domain-containing protein 11; CBM, CARD11-BCL-10-MALT1; IKK, inhibitor of NF-KB. The asterisks indicate recurrently mutated protein regions in human lymphoma. Figure taken from (Young and Staudt, 2013).

Furthermore, PI3K is activated through BCR crosslink and catalyzes the phosphorylation of phosphatidylinositol-4,5-biphosphate (PIP2) to phosphatidylinositol-3,4,5-triphosphate (PIP3), which acts as docking site for Akt (also known as protein kinase B, PKB) and phosphoinositide-dependent kinase 1 (PDK1). PDK1 phosphorylates Akt at threonine-308 in its kinase domain and subsequent phosphorylation of serine-473 by the mTor-rictor complex (mTORC2) leads to full activation of Akt (Sarbassov et al., 2005). Akt is the main mediator of the PI3K pathway. Through subsequent activation of mTor and resulting phosphorylation of ribosomal S6 kinases as well as the eukaryotic initiation factor 4E (elF4E)-binding protein 1, components of ribosomal biogenesis and mRNA translation, protein synthesis and cell growth are promoted (reviewed in Markman et al., 2010). In 
addition, Akt influences the transcription factor FOXO (forkhead box transcription factor), thus inactivating cell cycle inhibitors and promoting cellular proliferation (reviewed in Burgering and Medema, 2003). PI3K activity is counteracted by phosphatase and tensin homologue (PTEN), which is a PIP 3 3-phosphatase (Di Cristofano et al., 1998; Salmena et al., 2008). Aberrant activation of the PI3K/Akt-pathway can be found in a wide array of cancers, including hematological malignancies (reviewed in Chalhoub and Baker, 2009; Markman et al., 2010).

In contrast to the 'activated', antigen-induced B cell receptor signaling, a second form of BCR signaling mainly utilized by mature B cells to ensure B cell survival exists: so-called 'tonic' BCR signaling (Lam et al., 1997). Although several investigations implicate the PI3K pathway as a key component of tonic BCR signaling, the biochemical and biophysical basis of tonic BCR signaling and PI3K involvement is still debated (Cambier and Johnson, 1995; Delgado et al., 2009; Kraus et al., 2004; Lam et al., 1997; Monroe, 2006; Wienands et al., 1996).

BCR expression is maintained on the cell surface of most B cell lymphomas (reviewed in Küppers, 2005), although immunoglobulin loci are disrupted in favor of placing oncogenes under the control of the immunoglobulin enhancers (reviewed in Nussenzweig and Nussenzweig, 2010). Many B cell lymphomas utilize IgM constant regions to form their BCR, because IgM-BCR signaling seems to promote the survival and proliferation of B cells, instead of IgG-BCR signaling, which favors differentiation into plasma B cells (Dogan et al., 2009; Horikawa et al., 2007; Martin and Goodnow, 2002).

\subsubsection{CD40 mediated signaling}

CD40, a member of the TNFR (tumor necrosis factor receptor) superfamily, is expressed on the cellular surface of virtually all mature B lymphocytes. Upon binding to its ligand CD40L/CD154, CD40 activates multiple signaling pathways promoting B cell activation, proliferation and survival (reviewed in Van Kooten and Banchereau, 2000). CD40L is primarily expressed by activated (CD4 $\left.{ }^{+}\right) \mathrm{T}$ cells. The CD40-CD40L interaction is regarded as the 'classical' co-stimulatory signal, contributing to GC formation, memory B cell formation, Ig isotype switching and affinity maturation (DiSanto et al., 1993; Kawabe et al., 1994; Xu et al., 1994). A soluble form of CD40L exhibiting comparable activating features as the membrane-bound ligand has been reported as well (Graf et al., 1995; Mazzei et al., 1995).

Upon CD40-CD40L binding a multi-component protein complex is assembled, containing the adaptor molecules TNF receptor-associated factor 2 and 3 (TRAF2, TRAF3), ubiquitin conjugating enzyme Ubc13, cellular inhibitor of apoptosis proteins 1 and 2 (c-IAP1/2), IKKY/NEMO and MAPK kinase kinase 1 (MEKK1) (Matsuzawa et al., 2008). c-IAP1/2 mediated degradation of TRAF3 leads to 
translocation of the 'cytokine receptor-assembled signaling complex' to the cytosol and thus to the activation of MEKK1-mediated MAPK signaling via p38 and JNK (Gallagher et al., 2007). In addition IKK-promoted canonical and non-canonical NF-KB activation can occur. Moreover, recruitment of TRAF6 and Tak1 to CD40 upon its engagement was also suggested, providing a second mechanism to activate MAPK and NF-KB signaling in response to CD40 activation (Matsuzawa et al., 2008).

As in normal B cells, CD40 activation induces in certain B cell malignancies an increase in antiapoptotic factors such as Bcl-XL (B cell lymphoma-extra large), A20 (tumor necrosis factor, alphainduced protein 3 - TNFAIP3), survivin and CFLAR (CASP8- and FADD-like apoptosis regulator) (reviewed in Elgueta et al., 2009). Some in vitro investigations suggested that low-level constitutive engagement of CD40 may facilitate malignant cell growth. Studies with non-Hodgkin's lymphoma cells including Burkitt's lymphoma cells have shown that these cells express low levels of CD40L, which in turn leads to sustained cell proliferation through autocrine pathway activation and protection from apoptosis (Challa et al., 2002; Pham et al., 2002). Conversely, transient in vitro and in vivo activation of CD40 in Burkitt's lymphoma, multiple myeloma and primary high-grade B cell lymphoma resulted in reduction of tumor cell proliferation (Funakoshi et al., 1994; PellatDeceunynck et al., 1996). Thus, not only the activation status but also the quantitative level of CD40 activation might play a role in cell fate determination.

\subsubsection{Hedgehog signaling pathway}

The Hedgehog $(\mathrm{Hh})$ signaling pathway was first identified in 1980 in Drosophila melanogaster as essential developmental signaling pathway regulating proliferation, differentiation and migration of embryonic cells (Nüsslein-Volhard and Wieschaus, 1980). In humans, the Hh family of proteins comprises three distinct ligands which are processed from the same precursor protein: Sonic hedgehog (Shh), Indian hedgehog (Ihh) and Desert hedgehog (Dhh). In the absence of Hh ligands, the transmembrane receptor Patched1 (Ptch1) inhibits the activation of Smoothened (Smo), a transmembrane G-like protein coupled receptor and thereby prevents active Hh signaling. Once Hh binds to Ptch, the inhibition of Smo is abated, thus allowing Smo to translocate to the cytoplasm. Smo activation triggers a cascade of signaling events, leading to the activation of members of the glioma-associated oncoproteins (Gli) family of zinc-finger transcription factors (Gli1 and Gli2, which function mostly as activators while Gli3 as repressor). Gli then translocates to the nucleus and initiates transcription of $\mathrm{Hh}$ target genes, including GLI1, GLI2 and PTCH1 as well as regulators of cell proliferation and survival (Ikram et al., 2004; Lee et al., 1997). The exact details of these processes still have to be further investigated for mammalian cells. Since all Hh signaling via the canonical pathway require Smo, small molecule inhibitors as cyclopamine, which block Smo function, 
completely abrogate all Hh signaling regardless of the ligand (Incardona et al., 1998; Taipale et al., 2000).

The identification of point mutations in PTCH1 and SMO in patients with medulloblastomas, basal cell carcinomas and rhabdomyosarcomas (Aszterbaum et al., 1999; Goodrich and Scott, 1998; Marino, 2005; Xie et al., 1998) has linked the Hh signaling pathway to cancer. Recent investigations suggest that several cancers including B cell lymphoma abnormally express Hh ligands and activate the Hh signaling pathway in an autocrine manner (Singh et al., 2010). In addition to Shh production by follicular dendritic cells in the lymph node and its necessity for proliferation, survival and antibody production of germinal center B cells (Sacedón et al., 2005), another study demonstrated in vitro and in vivo secretion of Hh ligands by stromal cells in non-Hodgkin lymphoma, which promoted survival of the malignant cells in an inverse paracrine manner (Dierks et al., 2007).

\subsubsection{NF-kB signaling pathway}

The Rel/NF-KB-family of transcription factors plays a central role in the immune system by regulating various processes, including development and survival of $B$ and $T$ cells, as well as the control of immune responses and malignant transformation (reviewed in Vallabhapurapu and Karin, 2009). The NF-KB family comprises five subunits: NF-KB1 (p50 and its precursor p105), NF-KB2 (p52 and its precursor p100), p65 (RelA), c-Rel and RelB. These subunits form homo- and heterodimeric complexes that can bind to DNA and regulate NF-KB specific target genes. Inhibitors of NF-KB (IKB $\alpha$, IKB $\beta$ and $І \mathrm{~KB} \varepsilon$ ) hold NF-KB complexes in an inactive state in the cytoplasm (reviewed in Yamamoto and Gaynor, 2004). The release of NF-KB subunits from the IKBs is controlled by ІкB kinases (IKK $\alpha /$ IKK1, IKK $\beta /$ IKK2 and IKK $/$ NEMO) in a signal dependent manner. Many different stimuli activate the NF-KB transcription factors to induce their nuclear translocation. Here, two different pathways exist: the classical (canonical) and the alternative (non-canonical) pathway. The major and most-well studied pathway used by most stimuli (including antigen receptors (see chapter 1.2.1 for more detailed description), tumor necrosis factor receptors as CD40 (chapter 1.2.2), toll-like receptors (TLRs), IL-1R and others) is the canonical NF-KB pathway, which mainly employs the p65:p50 and cRel:p50 heterodimers. This pathway mainly centers on activation through a trimeric IKK-complex, where IKK1 and IKK2 function as catalytic subunits that phosphorylate the IKBs leading to their subsequent degradation, while IKK $\gamma /$ NEMO comprises the regulatory subunit of the IKK complex. The IKK2 subunit is referred to exhibit the majority of IKB kinase activity in most cell lines. In the absence of IKK2, IKK1 can adopt residual IKB kinase activity (Li et al., 1999), whereas deletion of IKK1 in IKK2expressing cells has nearly no effect on classical IKK activity (Hu et al., 1999). An important and unique function of IKK1 is the activation of the alternative NF-KB signaling pathway by activation through NIK (NF-KB-inducing kinase) (Senftleben et al., 2001). The non-canonical pathway is based on 
processing of the p100 precursor and subsequent activation of RelB:p52 dimers (Yilmaz et al., 2003). This alternative pathway is activated in response to a small subset of tumor necrosis factor (TNF) family members, including CD40L, but also regulated through the canonical NF-KB pathway, which upregulates NF-kB2 expression (Senftleben et al., 2001).

As mentioned above, NF-KB signaling is induced through a variety of different stimuli and positively as well as negatively regulated. Thus, it is not surprising that deregulated NF-KB signaling leads to aberrant expression of its target genes, which predominantly regulate cell survival, proliferation or growth and occupies an important role in many types of cancer. Hence, constitutively active NF-KB signaling can be found in several lymphoma subtypes including ABC (activated B celllike) DLBCL (see also chapter 1.3.1) or Epstein-Barr virus positive Burkitt's lymphoma (chapter 1.3.2).

\subsubsection{Mitogen activated protein kinase (MAPK) signaling pathways}

Mitogen activated protein kinases (MAPKs) are serine/threonine-specific protein kinases directing the cellular response to a diverse array of stimuli, such as mitogens, pro-inflammatory cytokines or stress signals. Amongst others they regulate proliferation, gene expression, differentiation, mitosis, cell survival and apoptosis (reviewed by Pearson, 2001). The best studied MAPKs are extra-cellular signal related kinases 1 and 2 (Erk1/2), the Jun-N-terminal kinases (JNKs) and p38 kinases. MAPKs are activated by phosphorylation cascades. The two upstream protein kinases MAPK kinase kinases (MKKs) and MKK kinases (MAP3Ks) are activated in series and regulate MAPKs. Furthermore, additional kinases may be required upstream of this three-kinase module. Erk1/2, JNK and p38 are predominantly regulated by different MKKs (Raman et al., 2007), thus resulting in distinct responses to a specific stimulus. Another characteristic of the phosphorylation cascade is signal amplification provided if the successive protein is more abundant than its regulator. In case of the Erk1/2 pathway, Raf and MEK1/2 are the relevant upstream protein kinases. MEK1/2 inhibitors have been used extensively to elucidate the biological relevance of Erk1/2 in a wide array of events. One of the most often used inhibitors is U0126 (Favata et al., 1998), which interacts preferentially with the inactive, unphosphorylated kinase, thus preventing activation of MEK1/2. Upon stimulation Erk1/2 phosphorylates a large number of substrates (reviewed in Yoon and Seger, 2006), among others the transcription factor Elk-1, which is thereby activated and is known to induce expression of immediate early response genes such as c-Fos (Gille et al., 1995).

In contrast to the relatively well-insulated Erk1/2 pathway, p38 and JNK share most of their activators at the MAP3K level (MEKK1, MEKK4, Tak1 and others). In addition, some of the MKKs directly activate both p38 and JNK (MKK4), whereas others are specific for either p38 (MKK3 and MKK6) or JNK (MKK7). Due to this interlacing there are few stimuli that can induce JNK activation without simultaneously regulating p38 or vice versa (Cargnello and Roux, 2011). Identification of the 
p38 $\alpha$-specific inhibitor SB203580 (Lee et al., 1994), which was exploited in a huge number of studies, revealed the pivotal functions of p38 in immune and inflammatory responses. P38 excerts its function through phosphorylation of many substrates in the cytoplasm and the nucleus, thereby modulating the activity of kinases as well as transcription factors (reviewed in Cuadrado and Nebreda, 2010).

To elucidate the role of JNK in inflammatory diseases, apoptotic cell death and cancer, the reversible ATP-competitive inhibitor SP600125 has been invented (Bennett et al., 2001). The transcription factor c-Jun is a well-known target of JNK, as well as other transcription factors including p53, Elk-1, JunB, Stat3 and c-Myc (reviewed in Weston and Davis, 2002).

Both MAPKs, p38 and JNK, are activated through the MAP3K Tak1 (transforming growth factor- $\beta$ activated kinase 1). Tak1 was originally identified as a protein kinase involved in TGF- $\beta$ (transforming growth factor $-\beta$ ) signaling (Yamaguchi et al., 1995), but has emerged in several studies for being activated through several external stimuli and thus being a key modulator of the cellular response to a variety of signals. These stimuli include IL-1R, TNF, bacterial lipopolysaccharide (LPS), CD40 and its viral counterpart LMP1, as well as BCR signaling in various types of cells (Arcipowski and Bishop, 2012; Irie et al., 2000; Ninomiya-Tsuji et al., 1999; Schuman et al., 2009; Shkoda et al., 2012; Sorrentino et al., 2008; Takaesu et al., 2012; Wan et al., 2004; Yamaguchi et al., 1995). 
Introduction

\subsection{Strategies to describe and stratify lymphoma cases based on gene expression profiling}

As described before, aNHL comprise a very heterogeneous group of malignancies. This is reflected in an increased number of distinct entities specified in the recent WHO classification (Campo et al., 2011; Jaffe, 2009; Swerdlow et al., 2008). The WHO classifications attempts to define disease entities according to their histology, morphology, clinical behavior and molecular characteristics to help clinicians and pathologists to recognize the specific entity. In the 1950s, all NHL diversities were pooled together and treated - clinically and experimentally - as one disease. With the help of the WHO classification and the rise of new molecular methods, multiple novel therapeutic options have emerged in the treatment of aNHL and the distinct subtypes of aNHL are treated differentially nowadays.

\subsubsection{Diffuse large B cell lymphoma (DLBCL)}

The most common subtype of B cell NHL in Western countries is the diffuse large B cell lymphoma, which comprises $25 \%$ to $35 \%$ of new cases annually (Cancer Research UK, 2010). Although historically assembled into one category both pathologically and clinically, patients with DLBCL vary tremendously in clinical presentation, prognosis and response to current therapies. Patients often present with a rapidly progressing lymphadenopathy. Standard chemotherapy regimen includes cyclophosphamide, doxorubicin, vincristine and prednisone and the monoclonal antibody rituximab (R-CHOP). Response rates in patients with low-risk disease range from $80 \%$ to $90 \%$ (Feugier et al., 2005), however the 5-year overall survival rate ranges between $30 \%$ and $50 \%$ in all DLBCL patients, indicating a large clinical spectrum of sensitivity to the standard treatment (reviewed in Cultrera and Dalia, 2012).

Advances in molecular diagnostics went along with the definition of prognostic factors for DLBCL. DLBCL tumors present with large B cells with a very high percentage of tumor infiltrate. Most of the tumor cells resemble germinal center centroblasts, carrying complex chromosomal aberrations (Dave et al., 2002). Chromosomal alterations leading to aberrant expression of proto-oncogenes as BCL-6, c-MYC, BCL-2 and P53 are associated with poor outcome (Friedberg, 2011). The most common chromosomal alteration, leading to constitutive activation, is the translocation of BCL-6, a critical transcriptional repressor, whose down regulation is essential for the exit from the germinal center reaction (Ci et al., 2008, 2009; Fukuda et al., 1997; Iqbal et al., 2007; Kusam et al., 2009; Parekh et al., 2007; Polo et al., 2004, 2007; Saito et al., 2009). 
Introduction

\section{Identification of several subgroups of DLBCLs by global gene expression profiling}

The availability of an ever-growing panel of new genetic technologies, including gene expression profiling, array comparative genomic hybridization, methylation profiling, miRNA profiling as well as deep sequencing has led to an exponential growth in the discovery of the genetic basics of DLBCL's heterogeneity. Based on gene expression profiling, Alizadeh and colleagues identified a stratification for DLBCLs subgroups established by their comparison of healthy B cells from distinct differentiation and activation statuses. Thereby two major molecular subtypes have emerged: germinal center B cell-like (GCB) DLBCL and activated B cell-like (ABC) DLBCL (Alizadeh et al., 2000). This classification helped to underline several diverse signaling properties of DLBCLs in further studies. Based on gene expression profiling, $A B C$ DLBCL were identified to be hallmarked by a constitutive active NF-KB signal (Lam et al., 2005). Subsequent studies confirmed the activity of NF-KB to be required for ABC DLBCL survival in contrast to GCB DLBCLs (Davis et al., 2001). Further studies revealed dependency of $A B C$ DLBCL cells on constitutive active BCR signaling, either in form of mutations in CARD11 (Ngo et al., 2006) or mutations affecting the ITAM motifs of CD79A and CD79B (Davis et al., 2010). Both mutations lead to constitutive activation of NF-KB through the CARD11-BCL10-MALT1 (CBM) complex (Bidère et al., 2009). Due to these very recent findings, $A B C D L B C L$ are now considered to be hallmarked by 'chronic active BCR signaling' (Davis et al., 2010) in contrast to other lymphoma entities which harbor tonic BCR signaling (compare chapter 1.2.1). Further on, the large number of downstream targets of NF-KB contribute to the poor prognosis of $\mathrm{ABC}$ DLBCL by collectively preventing apoptosis and rendering cells insensitive to chemotherapy (Baldwin, 2001; Rosenwald et al., 2002). By blocking the JAK signaling pathway in ABC DLBCL cells in vitro, resulting in apoptosis, it was shown that NF-KB signaling also induces cytokines, which act in an autocrine fashion resulting in activation of JAK/STAT signaling (Lam et al., 2008).

\subsubsection{Burkitt's lymphoma (BL)}

In the WHO's classification of lymphoid tumors, Burkitt's lymphoma (BL) is listed as an aggressive non-Hodgkin B cell lymphoma, characterized by a high proliferation rate of the malignant cells and deregulation of the $c-M Y C$ gene (Swerdlow et al., 2008). BL was first characterized by Denis Burkitt in 1958 as the most prevalent childhood lymphoma in Africa mostly localized at the jaw (Burkitt, 1958). Nowadays BL is classified in 3 clinical variants, differing in geographical distribution and Epstein-Barr virus (EBV) association. The endemic form of BL is found in equatorial Africa and is in $98 \%$ of cases associated with an EBV infection (Blum et al., 2004; van den Bosch, 2004; Brady et al., 2008; Ferry, 2006; Pattle and Farrell, 2006). The immunodeficiency-associated BL is related to an EBV infection in $30 \%$ of the cases and mainly presents in HIV carriers, where tumors can arise due to the severe immunosuppression coincident with the onset of AIDS (Powles et al., 2000). The sporadic form of BL, predominantly presented in Western countries, accounts for $2 \%$ of all lymphomas in European 
countries and the United States and is rarely associated with EBV infection (Blum et al., 2004; Brady et al., 2008; Ferry, 2006; Pattle and Farrell, 2006; Perkins and Friedberg, 2008). However, as the central leading event of pathogenesis all three forms share the activation of the $c-M Y C$ gene at $8 q 24$, usually through translocation with an immunoglobulin locus, which introduces a transcriptional enhancer element (reviewed in Hecht and Aster, 2000).

So far, for management of sporadic BL more intensive chemotherapy regimens are required than for treatment of DLBCLs (Blum et al., 2004; Magrath et al., 1996; Mead et al., 2002). Furthermore, because of the high risk of involvement of the central nervous system systemic chemotherapy crossing the blood-brain barrier is often essential (Bishop et al., 2000). The diagnosis of BL mainly relies on morphologic findings, cytogenetic features and immunophenotyping results (Jaffe, 2009; Swerdlow et al., 2008). However, the main diagnostic challenge in $B L$ is to distinguish it from DLBCL, which may present with similar histological features. Additionally, the characteristic $t(8 ; 14)$ translocation of BLs, juxtaposing the $c-M Y C$ gene with an immunoglobulin enhancer also occurs in 5 to 10 percent of DLBCL cases (Kramer et al., 1998). Since the prevalence for DLBCLs is 20 times higher than for $\mathrm{BL}$, a lymphoma presenting with a $\mathrm{t}(8 ; 14)$ translocation may raise diagnostic challenge.

\section{Molecular Diagnosis of BL with the index of 'Burkitt-likeness'}

In 2006, two major DNA microarray studies were published using whole genome gene expression profiling to distinguish BL from DLBCL on the molecular level (Dave et al., 2006; Hummel et al., 2006). Using a new statistical approach, Hummel and colleagues developed a molecular classifier to achieve a clear discrimination of $\mathrm{BL}$ from other mature aggressive B-NHL. Based on the gene expression profile of $220 \mathrm{NHL}$ tumors, they identified 58 genes that constituted a molecular Burkitt's lymphoma $(\mathrm{mBL})$ signature. This $\mathrm{mBL}$ signature was used to establish a continuous index score (I) of 'Burkittlikeness' that allows the stratification of aNHL cases either into $\mathrm{mBL}(\mathrm{I}>0.95)$, non-mBL $(I<0.05)$ or intermediate lymphomas $(0.95<1>0.05$ ) (Hummel et al., 2006). Array-based comparative genomic hybridization (CGH) further revealed that $\mathrm{mBLs}$ had very few genetic alterations in addition to an IGMYC translocation (simple karyotype), whereas intermediate and non-mBL cases displayed a higher genetic complexity (non-IG-MYC translocations and other genetic aberrations on the background of a complex karyotype). At the same time, Dave and colleagues established a classifier for Burkitt's lymphoma based on gene expression profiling with the help of c-Myc target genes identified by siRNA-mediated knock down of c-MYC in an ABC DLBCL cell line (OCI-Ly10). Tumor biopsies from Burkitt's lymphomas could be distinguished to a certain extend from DLBCLs by high expression of cMyc target genes, the expression of a subgroup of germinal-center B cell genes and decreased expression of NF-KB target genes and major-histocompatibility-complex (MHC) class I genes. In summary, four different gene expression profiles were used to distinguish BL from DLBCL, but the 
molecular mechanisms underlying those differences in gene expression are still unclear (Dave et al., 2006).

\subsubsection{Surrogate markers for oncogenic pathway activation}

Multiple studies demonstrated that DNA-microarray based gene expression profiling (GEP) provides the ability to define cancer subtypes, recurrence of disease and response to specific therapies. Various studies have also shown the potential of gene expression profiles for the analysis of oncogenic pathways. Hence, its stands to reason to use gene expression signatures, obtained through specified manipulation of target cells in vitro, to identify and model patterns of oncogene activity, which underlie the development of the oncogenic phenotype and reflect the biology and outcome of specific cancers (Bentink et al., 2008; Bild et al., 2006). Thus, the gene expression data established from ectopic expression of several oncogenes (including RAS, SRC, 6-catenin, E2F3 and MYC) in primary human epithelia cells (Bild et al., 2006) has been used to model gene modules, representing the activity of a defined proto-oncogene (Bentink et al., 2008). These data were then combined to so-called 'pathway activation patterns' (PAPs), which stratified mature aggressive B cell lymphomas into distinct groups. One of the identified PAPs comprised mainly aNHL cases, which were characterized as 'intermediate' by the $\mathrm{mBL}$ index and present mainly cases carrying a complex karyotype and being situated in the transitional zone between $\mathrm{mBL}$ and non-mBL (Hummel et al., 2006). The underlying pathway activities were largely undefined. In the meantime, GEP was used to demonstrate that outcome in DLBCL patients seems to be influenced by differences in stromal factors supplied by the microenvironment of the tumor, such as differences in immune cells, fibrosis and angiogenesis (Lenz et al., 2008).

Driven by these investigations, the group of Dr. Kube started to identify and characterize new gene expression patterns that harbor information about relevant oncogenic pathway activities. In order to better understand the pathogenesis, implicated diagnostic and therapeutic possibilities of lymphomas, gene expression profiling of lymphomas was used to reveal oncogenic pathway activities. Therefore, one study focused on the effect of aberrant MYC activity in lymphoma precursor cells and its role in aNHL. Schrader and colleagues could later on demonstrate that high cMyc activity constitutes a negative prognostic marker in DLBCLs independent of established risk factors and of the presence of a MYC translocation (Schrader et al., 2012a). Additionally, A. Schrader also investigated the possibility to identify novel pathway activities in malignant lymphoma by transferring gene expression data from in vitro perturbation assays performed in lymphoma cell lines to primary lymphoma gene expression profiles (Schrader, 2011) and thus was instrumental in preparing the present study. 


\section{Aim of the Study}

A major step fostering the understanding of the clinical heterogeneity of aNHL was the recent molecular classification of aNHL based on global gene expression analyses of individual lymphomas. These transcriptome based analyses aimed to describe oncogenic events to identify new biomarkers and potential targets for individualized therapies of aNHL. However, the mechanisms behind the current criteria to distinguish individual NHL subtypes are still poorly understood. A more functional characterization of deregulated pathways in aNHL needs to be elucidated in order to improve our knowledge of the molecular mechanisms of malignant transformation of B cells.

We postulate that the description of time-resolved global gene expression changes provides new insights into the dynamics of oncogenic signaling. Furthermore, the intervention into pathway intersections by chemical inhibitors will allow us to describe the pathways interplay and cross talk.

Based on in vitro experiments, it was concluded that subsets of aNHLs are characterized by aberrantly activated NF-KB and JAK-STAT signals. Using stimulation-mediated gene expression changes, specific pathway activities in individual NHL were predicted. Regardless these achievements, a number of open questions still exist and need to be answered.

1) How is the activation of distinct pathway networks by BCR and CD40 activation reflected in the gene expression changes occurring over time?

a. Identify groups of genes which are regulated in a comparable way in response to $B C R$ and CD40 activation.

b. Identify gene expression changes which reflect the immediate early and the late pathway activation triggered by BCR / CD40 activation.

c. Identify networks of genes which reflect a dependency of gene expression regulations and could thereby represent regulatory circuits or feedback loops that stand in causal relationships within oncogenic pathways.

2) Is the gene expression profile of DLBCL cells, in contrast to that of BL cells, dominantly shaped by constitutive active micromilieu signals?

a. Evaluate how sustained activation of micromilieu derived signals influences the Burkitt-likeness of human transformed germinal center B cells.

b. Interrogate whether it is possible to resemble the activated gene expression profile of DLBCL by stimulating BL cells in vitro.

3) What is the functional background of the observed pathway activities?

a. Interrogate which differentially regulated genes could be involved in the oncogenic function of the identified gene groups / signatures. 
b. Identify new functions of signaling molecules that are involved in mediating these pathway activities.

In order to answer these questions, a prototypical cell line characteristic for human transformed germinal center B cells was used to analyze the temporal pattern of global gene expression changes upon signaling pathway activation by factors of the microenvironment. Secondly, several potential oncogenic signaling pathways were inactivated at different levels in the signaling cascade in order to dissect intersections in the complex signaling network evolving in a lymphoma cell. 


\section{Material and Methods}

\subsection{Biological Material}

\subsubsection{Primary Material}

Primary material of human pediatric patients was obtained with consent and ethical approval from tonsillectomies performed in the University Medical Center Göttingen, Germany.

\subsubsection{Cell Lines}

Cell lines used in this work are listed in Table 2-1.

Table 2-1 Cell lines

\begin{tabular}{|c|c|c|c|}
\hline Cell line & Source & Distributor & Reference \\
\hline BL2 & $\begin{array}{l}\text { B cell, Burkitt Lymphoma } \\
\text { (EBV negative) }\end{array}$ & DSMZ & $\begin{array}{l}\text { (Kube et al., 1995; Nilsson and } \\
\text { Pontén, 1975) }\end{array}$ \\
\hline BL41 & $\begin{array}{l}\text { B cell, Burkitt Lymphoma } \\
\text { (EBV negative) }\end{array}$ & DSMZ & (Lenoir et al., 1985) \\
\hline BL70 & $\begin{array}{l}\text { B cell, Burkitt Lymphoma } \\
\text { (EBV negative) }\end{array}$ & DSMZ & (Lenoir et al., 1985) \\
\hline DG-75 & $\begin{array}{l}\text { B cell, Burkitt Lymphoma } \\
\text { (EBV negative) }\end{array}$ & DSMZ & (Ben-Bassat et al., 1977) \\
\hline HT & $\begin{array}{l}\text { B cell, Diffuse Large B cell } \\
\text { Lymphoma (GCB) }\end{array}$ & DSMZ & (Beckwith et al., 1990) \\
\hline Ramos & $\begin{array}{l}\text { B cell, Burkitt Lymphoma } \\
\text { (EBV negative) }\end{array}$ & DSMZ & (Klein et al., 1975) \\
\hline SuDHL4 & $\begin{array}{l}\text { B cell, Diffuse Large B cell } \\
\text { Lymphoma (GCB) }\end{array}$ & DSMZ & (Epstein et al., 1976) \\
\hline SuDHL5 & $\begin{array}{l}\text { B cell, Diffuse Large B cell } \\
\text { Lymphoma (GCB) }\end{array}$ & DSMZ & (Epstein et al., 1978) \\
\hline SuDHL6 & $\begin{array}{l}\text { B cell, Diffuse Large B cell } \\
\text { Lymphoma (GCB) }\end{array}$ & DSMZ & (Epstein et al., 1978) \\
\hline OCl-Ly1 & $\begin{array}{l}\text { B cell, Diffuse Large B cell } \\
\text { Lymphoma (GCB) }\end{array}$ & $\begin{array}{l}\text { Ontario Cancer } \\
\text { Institute, Toronto }\end{array}$ & (Chang et al., 1995) \\
\hline OCl-Ly3 & $\begin{array}{l}\text { B cell, Diffuse Large B cell } \\
\text { Lymphoma }(A B C)\end{array}$ & $\begin{array}{l}\text { Ontario Cancer } \\
\text { Institute, Toronto }\end{array}$ & (Tweeddale et al., 1987) \\
\hline
\end{tabular}




\begin{tabular}{|l|l|l|l|}
\hline OCI-Ly7 & $\begin{array}{l}\text { B cell, Diffuse Large B cell } \\
\text { Lymphoma (GCB) }\end{array}$ & $\begin{array}{l}\text { Ontario Cancer } \\
\text { Institute, Toronto }\end{array}$ & (Chang et al., 1995) \\
\hline U2932 & $\begin{array}{l}\text { B cell, Diffuse Large B cell } \\
\text { Lymphoma (ABC) }\end{array}$ & DSMZ & (Amini et al., 2002) \\
\hline
\end{tabular}

\subsection{Chemicals and Consumable Supplies}

Chemicals and consumables used in the present study are listed in Table 2-2 and Table 2-3.

Table 2-2 Chemicals

\begin{tabular}{|c|c|}
\hline Chemical & Manufacturer \\
\hline$\alpha$-Thiolglycerol & Sigma-Aldrich, Munich GER \\
\hline Acrylamid/Bisacrylamid $40 \%$ & BioRad, Munich GER \\
\hline Ammonium persulphate & Sigma-Aldrich, Munich GER \\
\hline Bradford solution & RotiQuant-Roth, Karlsruhe GER \\
\hline Bromphenol Blue & Sigma-Aldrich, Munich GER \\
\hline Bovine serum albumin (BSA) & Serva, Heidelberg GER \\
\hline Diethylpyrucarbonate (DEPC) & Roth, Karlsruhe GER \\
\hline Dimethyl sulfoxide (DMSO) & Sigma-Aldrich, Munich GER \\
\hline $\begin{array}{l}\text { deoxyribonucleoside triphosphates (dNTPs: } 100 \mathrm{mM} \\
\text { dATP, dCTP, dGTP, dTTP) }\end{array}$ & PrimeTech LTD, Minsk BY \\
\hline Ethanol (100 \%) & J.T. Baker, Deventer NL \\
\hline Ethylenediaminetetraacetic acid (EDTA) & Riedel-de Haën, Seelze GER \\
\hline $\begin{array}{l}\text { Full Range Rainbow Molecular Weight Marker } \\
\text { RPN800 }\end{array}$ & GE Healthcare, Munich GER \\
\hline Formaldehyde & Sigma-Aldrich, Munich GER \\
\hline Glycerol & Roth, Karlsruhe GER \\
\hline Glycine & Roth, Karlsruhe GER \\
\hline HEPES & Sigma-Aldrich, Munich GER \\
\hline Hot FIREpol DNA polymerase $(5 \mathrm{U} / \mu \mathrm{l})$ & PrimeTech LTD, Minsk BY \\
\hline Hydrogen Peroxide & Sigma-Aldrich, Munich GER \\
\hline
\end{tabular}




\begin{tabular}{|c|c|}
\hline Isopropanol & Sigma-Aldrich, Munich GER \\
\hline Luminol & Sigma-Aldrich, Munich GER \\
\hline Magnesium chloride (25 mM) & PrimeTech LTD, Minsk BY \\
\hline Methanol $100 \%$ (р.а.) & J.T. Baker, Deventer NL \\
\hline Milk powder & Roth, Karlsruhe GER \\
\hline Nonidet P-40 & Sigma-Aldrich, Munich GER \\
\hline Phosphatase inhibitor Phospho- STOP & Roche, Mannheim GER \\
\hline Phenylmethylsulfonyl fluoride (PMSF) & Sigma-Aldrich, Munich GER \\
\hline Proteaseinhibitor-Mix Complete ${ }^{\mathrm{TM}}$ & Santa Cruz biotechnology, Heidelberg GER \\
\hline RPMI-1640 & Lonza, Basel CH \\
\hline Sodium dodecyl sulfate (SDS) & Merck, Darmstadt, GER \\
\hline Sodium chloride & Merck, Darmstadt, GER \\
\hline Sodium deoxycholate & Merck, Darmstadt, GER \\
\hline Sodiumvanadat & Sigma-Aldrich, Munich GER \\
\hline Spectra Multicolor Broad Range Protein Ladder & Fermentas, Frankfurt GER \\
\hline SYBR Green I Nucleic Acid Gel Stain & Roche, Grenzach GER \\
\hline Tetramethylethylenediamine (TEMED) & Sigma-Aldrich, Munich GER \\
\hline Trehalose & Roth, Karlsruhe GER \\
\hline Trisbase & Sigma-Aldrich, Munich GER \\
\hline TritonX-100 & Roth, Karlsruhe GER \\
\hline Trypanblue $0.4 \%$ in PBS & GIBCO BRL, Life Technologies, Eggenstein GER \\
\hline Tween-20 & Merck, Darmstadt GER \\
\hline Water HPLC grade & Merck, Darmstadt GER \\
\hline
\end{tabular}


Table 2-3 Consumables

\begin{tabular}{|c|c|}
\hline Consumables & Manufacturer \\
\hline ABI PRISM ${ }^{\circledR}$ 384-Well Clear Optical Reaction Plate & Applied Biosystems, Foster City USA \\
\hline ABI PRISM ${ }^{\circledR}$ Optical Adhesive Covers & Applied Biosystems, Foster City USA \\
\hline Cell culture flasks & Sarstedt, Nümbrecht GER \\
\hline Cryo Box & Nunc, Wiesbaden GER \\
\hline Cryotubes & Nunc, Wiesbaden GER \\
\hline Diethylaminoethyl-Cellulose & Whatman $^{\circledR}$, International Ltd UK \\
\hline Electroporation Cuvettes & BioRad, Munich GER \\
\hline Falcon Tubes $15 \mathrm{ml}$ & Sarstedt, Nümbrecht GER \\
\hline Falcon Tubes $50 \mathrm{ml}$ & Sarstedt, Nümbrecht GER \\
\hline Filter Tips, $10 \mu \mathrm{l}, 100 \mu \mathrm{l}, 200 \mu \mathrm{l}, 1000 \mu \mathrm{l}$ & Starlab, Ahrensburg GER \\
\hline Micro Touch Examination Gloves & Ansell, München GER \\
\hline Pasteurpipettes & Sarstedt, Nümbrecht GER \\
\hline Pipette Tips w/o filters & Sarstedt, Nümbrecht GER \\
\hline Reaction tubes $0.5 \mathrm{ml}, 1.5 \mathrm{ml}, 2.0 \mathrm{ml}$ & Sarstedt, Nümbrecht GER \\
\hline Serological pipettes $5 \mathrm{ml}, 10 \mathrm{ml}, 25 \mathrm{ml}$ & Sarstedt, Nümbrecht GER \\
\hline Tissue culture dish & Sarstedt, Nümbrecht GER \\
\hline Tissue culture plates, 6 well, 12 well, 24 well & Nunc, Wiesbaden GER \\
\hline 96 well plate, Round/Flat bottom & Nunc, Wiesbaden GER \\
\hline
\end{tabular}




\subsection{Buffers, Solutions and Media}

Buffers, solutions and media used for this study are listed in Table 2-4.

Table 2-4 Buffers, solutions and media

\begin{tabular}{|c|c|}
\hline Liquid & Receipt / Manufacturer \\
\hline $4 \times$ loading buffer Roti ${ }^{\circledR}$-Load & Roth, Karlsruhe GER \\
\hline $\begin{array}{l}\text { Cell culture medium I } \\
\text { (cell lines - BL) }\end{array}$ & $\begin{array}{l}\text { RPMI-1640 } \\
10 \% \text { (v/v) FBS (Sigma-Aldrich, Munich GER) } \\
200 \mathrm{U} / \mathrm{ml} \text { Penicilin + } 200 \mu \mathrm{g} / \mathrm{ml} \text { Streptomycin + } \\
4 \mathrm{mM} \text { L-Glutamine (PSG) (Sigma-Aldrich, Munich GER) } \\
50 \mu \mathrm{M} \alpha \text {-Thiolglycerol, } 20 \mathrm{nM} \mathrm{BCS,} 1 \mathrm{mM} \text { Sodium pyruvat }\end{array}$ \\
\hline $\begin{array}{l}\text { Cell culture medium II } \\
\text { (cell lines - DLBCL) }\end{array}$ & $\begin{array}{l}\text { RPMI-1640 } \\
10 \%(\mathrm{v} / \mathrm{v}) \text { FBS } \\
200 \mathrm{U} / \mathrm{ml} \text { Penicilin }+200 \mu \mathrm{g} / \mathrm{ml} \text { Streptomycin + } \\
4 \mathrm{mM} \text { L-Glutamine (PSG) }\end{array}$ \\
\hline Cell culture medium III & $\begin{array}{l}\text { IMDM (PAA) } \\
10 \%(\mathrm{v} / \mathrm{v}) \text { FBS } \\
200 \mathrm{U} / \mathrm{ml} \text { Penicilin }+200 \mu \mathrm{g} / \mathrm{ml} \text { Streptomycin + } \\
4 \mathrm{mM} \text { L-Glutamine (PSG) } \\
\text { OCI-Ly3 cells were cultivated with } 20 \% \text { FBS }\end{array}$ \\
\hline EDTA cell culture grade & $0.5 \mathrm{M}$ (Sigma Aldrich, Munich GER) \\
\hline Freezing medium & $\begin{array}{l}90 \%(v / v) \text { FCS } \\
10 \%(v / v) \text { DMSO }\end{array}$ \\
\hline Nuclear Extract Buffer A & $\begin{array}{l}10 \text { mM HEPES pH } 7.9 \\
10 \mathrm{mM} \mathrm{KCl} \\
10 \mu \mathrm{M} \text { EDTA } \\
10 \mu \mathrm{M} \text { EGTA } \\
1 \mathrm{mM} \text { DTT } \\
1 \times \text { Complete Mini Protease Inhibitor } \\
1 \text { x PhosStop }\end{array}$ \\
\hline
\end{tabular}




\begin{tabular}{|c|c|}
\hline Nuclear Extract Buffer B & $\begin{array}{l}20 \text { mM HEPES pH } 7.9 \\
400 \mathrm{mM} \mathrm{KCl} \\
1 \mathrm{mM} \text { EDTA } \\
1 \mathrm{mM} \text { EGTA } \\
1 \mathrm{mM} \text { DTT } \\
1 \text { x Complete Mini Protease Inhibitor } \\
1 \text { x PhosStop }\end{array}$ \\
\hline PCR buffer (10x) for qRT & $\begin{array}{l}750 \mathrm{mM} \text { Tris-HCl pH } 8.8 \\
200 \mathrm{mM} \text { Ammonium sulfate } \\
0.1 \% \text { Tween-20 } \\
\text { in depc water }\end{array}$ \\
\hline $\begin{array}{l}\text { PBS } \\
\text { (Phosphate Buffered Saline) }\end{array}$ & $\begin{array}{l}137 \mathrm{mM} \mathrm{NaCl} \\
10 \mathrm{mM} \text { Phosphate } \\
2.7 \mathrm{mM} \mathrm{KCl}, \mathrm{pH} 7.4 .\end{array}$ \\
\hline PBS pH 7.4 (cell culture grade) & Lonza, Verviers BEL \\
\hline Ponceau-S & $\begin{array}{l}5 \%(\mathrm{v} / \mathrm{v}) \text { glacial acetic acid } \\
0.5 \%(\mathrm{w} / \mathrm{v}) \text { Ponceau-S }\end{array}$ \\
\hline ReBlot plus mild & Millipore, Schwalbach/Ts. GER \\
\hline RIPA buffer & $\begin{array}{l}150 \mathrm{mM} \mathrm{NaCl} \\
1 \% \mathrm{NP}-40 \\
0.1 \% \mathrm{SDS} \\
50 \mathrm{mM} \text { TrisHCl pH 7,4 } \\
0.25 \% \text { Sodium-Deoxycholat }\end{array}$ \\
\hline Running buffer (1x): & $\begin{array}{l}25 \mathrm{mM} \text { Tris-Base } \\
192 \mathrm{mM} \text { Glycin } \\
34.67 \mathrm{mM} \text { SDS }\end{array}$ \\
\hline Separation Gel Mix & $\begin{array}{l}31.3 \% \text { (v/v) Acrylamid/Bis Solution (40 \%) } \\
332 \mathrm{mM} \text { Tris Base, } \mathrm{pH} 8.9 \\
3.33 \mathrm{mM} \text { EDTA }\end{array}$ \\
\hline Stacking Gel Mix & $\begin{array}{l}15 \% \text { (v/v) Acrylamid/Bis Solution ( } 40 \% \text { ) } \\
125 \mathrm{mM} \text { Tris Base } \mathrm{pH} 6.8 \\
0.1 \%(\mathrm{w} / \mathrm{v}) \text { SDS } \\
5 \mathrm{mM} \text { EDTA }\end{array}$ \\
\hline
\end{tabular}




\begin{tabular}{|c|c|}
\hline $\begin{array}{l}\text { Solution } 1 \text { Chemiluminescence - } \\
\text { Luminol }\end{array}$ & $\begin{array}{l}100 \mathrm{mM} \text { Tris/HCl pH } 8.8 \\
2.5 \mathrm{mM} \text { Luminol (Sigma-Aldrich, Munich GER) } \\
4 \mathrm{mM} \text { 4-IPBA (Sigma-Aldrich, Munich GER) }\end{array}$ \\
\hline $\begin{array}{l}\text { Solution } 2 \text { Chemiluminescence - } \\
\text { Peroxide }\end{array}$ & $\begin{array}{l}100 \mathrm{mM} \text { Tris/HCl pH } 8.8 \\
10.6 \mathrm{mM} \mathrm{H} 2 \mathrm{O} 2\end{array}$ \\
\hline SybrGreenMix & $\begin{array}{l}1 \times \text { PCR buffer } \\
3 \mathrm{mM} \mathrm{MgCl} 2 \\
\text { 1:80000 SybrGreen } \\
0.2 \mathrm{mM} \text { dNTP each } \\
20 \mathrm{U} / \mathrm{ml} \text { Hot FIREpol DNA polymerase } \\
0.25 \% \text { TritonX-100 } \\
0.5 \mathrm{mM} \text { Trehalose in depc water }\end{array}$ \\
\hline TBS (1x) & $\begin{array}{l}20 \text { mM Tris-Base, } \\
137 \text { mM Sodium Chloride, pH } 7.6\end{array}$ \\
\hline TBS-T & $\begin{array}{l}1 \times \text { TBS } \\
0.1 \%(v / v) \text { Tween-20 }\end{array}$ \\
\hline Transferbuffer (1x): & $\begin{array}{l}25 \mathrm{mM} \text { Tris-Base } \\
192 \mathrm{mM} \text { Glycin } \\
15 \%(\mathrm{v} / \mathrm{v}) \mathrm{MeOH}\end{array}$ \\
\hline
\end{tabular}

\subsection{Equipment}

Table 2-5 lists the equipment used in the present study.

Table 2-5 Equipment

\begin{tabular}{|l|l|}
\hline Instrument & Manufacturer \\
\hline ABI PRISM 7900HT & Applied Biosystems, Foster City USA \\
\hline Accu-jet & Brand, Hamburg GER \\
\hline Biofuge Pico & Heraeus Instruments, Hanau GER \\
\hline Biofuge Primo R & Heraeus Instruments, Hanau GER \\
\hline Consort E734 Power Supply & Schütt Labortechnik, Göttingen GER \\
\hline CAT RM 5 horizontal roller & CAT M Zipperer, Staufen GER \\
\hline
\end{tabular}




\begin{tabular}{|c|c|}
\hline Centrifuge 5451D & Eppendorf, Hamburg GER \\
\hline GenePulser Xcell & Bio-Rad, Munich GER \\
\hline Hera freeze $-80^{\circ} \mathrm{C}$ freezer & Heraeus Instruments, Hanau GER \\
\hline IKA KS 260 shaker & IKA, Staufen GER \\
\hline IKAMAG RCT magnetic stirrer & IKA, Staufen GER \\
\hline Incubator Cytoperm & Heraeus Instruments, Hanau GER \\
\hline Incudrive incubator & Schütt Labortechnik, Göttingen GER \\
\hline LAS-4000 Image Reader & Fujifilm, Düsseldorf GER \\
\hline Microflow Laminar Downflow Workstation & Bioquell, UK \\
\hline Microcoolcentrifuge 1-15k & Sigma, Munich GER \\
\hline Multifuge $3 \mathrm{~L}-\mathrm{R}$ & Heraeus Instruments, Hanau GER \\
\hline ND-1000 UV/Vis-Spectrophotometer & NanoDrop ${ }^{\mathrm{TM}}$, Wilmington USA \\
\hline Neubauer Counting Chamber Improved & Lo Labor Optik, Friedrichsdorf GER \\
\hline Power Pac 300 Power Supply & Bio-Rad, München GER \\
\hline Reax2 shaker & Heidolph, Schwabach GER \\
\hline SunriseTM Microplate Reader & Tecan, Crailsheim GER \\
\hline Thermocycler Mastercycler & Eppendorf, Hamburg GER \\
\hline Thermocycler 60 & Biomed, Theres GER \\
\hline Thermocycler T3000 & Biometra, Göttingen GER \\
\hline Thermomixer Compact & Eppendorf, Hamburg GER \\
\hline Vortex Genie 2 & Schütt Labortechnik, Göttingen GER \\
\hline Water bath & Köttermann Labortechnik, Hänigsen GER \\
\hline
\end{tabular}

\subsection{Recombinant Proteins}

BL and DLBCL cell lines as well as primary B cells were stimulated with either SCD40L (\#ABC159; AutogenBioclear, Calne, Wiltshire UK) or goat anti human IgM F(ab) 2 fragment (\#109006129; Jackson ImmunoResearch / Dianova, Hamburg GER). 


\subsection{Inhibitors}

The chemical inhibitors used in this study are listed in Table 2-6. The final concentration listed for each inhibitor was used, if not stated differentially in the text.

Table 2-6 Chemical inhibitors

\begin{tabular}{|l|l|l|}
\hline Description (target) & Manufacturer & Final concentration \\
\hline 10058-F4 (c-Myc) & Sigma-Aldrich, Munich GER & $60 \mu \mathrm{M}$ \\
\hline 5Z-7-oxozeaenol (TAK1/MAP3K7) & Calbiochem/Merck, Darmstadt GER & $100 \mathrm{nM}$ \\
\hline Akti-1/2 (Akt) & Calbiochem/Merck, Darmstadt GER & $2.5 \mu \mathrm{M}$ \\
\hline AZ-Tak1 (TAK1) & Tocris Biosciences, Toronto, CA & $500 \mathrm{nM}$ \\
\hline Bisindolylmaleimide I (PKC) & Calbiochem/Merck, Darmstadt GER & $1 \mu \mathrm{M}$ \\
\hline BKM-120 (PI3K) & Selleckchem, Munich GER & $1 \mu \mathrm{M}$ \\
\hline BX-912 (PDK1) & Selleckchem, Munich GER & $250 \mathrm{nM}$ \\
\hline Compound 15e (PI3K) & Enzo Life Sciences, Lörrach GER & $400 \mathrm{nM}$ \\
\hline Cyclopamine (SMO) & Calbiochem/Merck, Darmstadt GER & $5 \mu \mathrm{M}$ \\
\hline Everolimus (mTOR) & Sigma-Aldrich, Munich GER & $1 \mu \mathrm{M}$ \\
\hline IKK2 inhibitor VIII (IKK2) & Calbiochem/Merck, Darmstadt GER & $7 \mu \mathrm{M}$ \\
\hline Ly294002 (PI3K) & Calbiochem/Merck, Darmstadt GER & $10 \mu \mathrm{M}$ \\
\hline SB203580 (p38/MAPK14) & Sigma-Aldrich, Munich GER & $2 \mu \mathrm{M}$ \\
\hline SP600125 (JNK) & Calbiochem/Merck, Darmstadt GER & $10 \mu \mathrm{M}$ \\
\hline U0126 (MEK1/2) & Sigma-Aldrich, Munich GER & $10 \mu \mathrm{M}$ \\
\hline U73122 (PLCY) & Tocris Biosciences, Toronto CA & $1 \mu \mathrm{M}$ \\
\hline
\end{tabular}




\subsection{Antibodies}

Antibodies used for immunoblotting are summarized in Table 2-7.

Table 2-7 Antibodies used for immunoblotting.

\begin{tabular}{|c|c|c|}
\hline Antibody & Source & Working dilution \\
\hline $\begin{array}{l}\text { mouse monoclonal anti } \alpha- \\
\text { tubulin (\#05-829) }\end{array}$ & $\begin{array}{l}\text { Upstate/Millipore, Schwalbach } \\
\text { GER }\end{array}$ & $\begin{array}{l}\text { 1:5000 in } 3 \% \text { milkpowder in } \\
\text { TBS-T }\end{array}$ \\
\hline rabbit anti p-AKT (S473) (\#9271) & $\begin{array}{l}\text { Cell Signalling/ New England } \\
\text { Biolabs, Frankfurt am Main GER }\end{array}$ & $1: 1000$ in $5 \%$ BSA in TBS-T \\
\hline $\begin{array}{l}\text { rabbit anti p-AKT (S473) (D9E) } \\
(\# 4060)\end{array}$ & $\begin{array}{l}\text { Cell Signalling/ New England } \\
\text { Biolabs, Frankfurt am Main GER }\end{array}$ & 1:1000 in $5 \%$ BSA in TBS-T \\
\hline rabbit p-Akt (T308) (C31E5E) & $\begin{array}{l}\text { Cell Signalling/ New England } \\
\text { Biolabs, Frankfurt am Main GER }\end{array}$ & 1:1000 in $5 \%$ BSA in TBS-T \\
\hline $\begin{array}{l}\text { rabbit anti pan AKT (C67E7) } \\
(\# 4691)\end{array}$ & $\begin{array}{l}\text { Cell Signalling/ New England } \\
\text { Biolabs, Frankfurt am Main GER }\end{array}$ & 1:1000 in $5 \%$ BSA in TBS-T \\
\hline rabbit anti c-myc (\#9402) & $\begin{array}{l}\text { Cell Signalling/ New England } \\
\text { Biolabs, Frankfurt am Main GER }\end{array}$ & $1: 1000$ in $5 \%$ BSA in TBS-T \\
\hline rabbit anti c-Rel (G57) (\#4774) & $\begin{array}{l}\text { Cell Signalling/ New England } \\
\text { Biolabs, Frankfurt am Main GER }\end{array}$ & $1: 1000$ in $5 \%$ BSA in TBS-T \\
\hline rabbit anti HDAC1 (\#2062) & $\begin{array}{l}\text { Cell Signalling/ New England } \\
\text { Biolabs, Frankfurt am Main GER }\end{array}$ & 1:1000 in $5 \%$ BSA in TBS-T \\
\hline $\begin{array}{l}\text { rabbit anti } p-p 38 \\
(\mathrm{~T} 180 / \mathrm{Y} 182)(\# 9211)\end{array}$ & $\begin{array}{l}\text { Cell Signalling/ New England } \\
\text { Biolabs, Frankfurt am Main GER }\end{array}$ & $1: 1000$ in $5 \%$ BSA in TBS-T \\
\hline rabbit anti p38 (\#9212) & $\begin{array}{l}\text { Cell Signalling/ New England } \\
\text { Biolabs, Frankfurt am Main GER }\end{array}$ & $1: 1000$ in $5 \%$ BSA in TBS-T \\
\hline $\begin{array}{l}\text { rabbit anti } p-p 44 / p 42(p- \\
\text { ERK1/2) (\#4372) }\end{array}$ & $\begin{array}{l}\text { Cell Signalling/ New England } \\
\text { Biolabs, Frankfurt am Main GER }\end{array}$ & $1: 1000$ in $5 \%$ BSA in TBS-T \\
\hline $\begin{array}{l}\text { rabbit anti p44/p42 (ERK1/2) } \\
\text { (\#4695) }\end{array}$ & $\begin{array}{l}\text { Cell Signalling/ New England } \\
\text { Biolabs, Frankfurt am Main GER }\end{array}$ & 1:1000 in $5 \%$ BSA in TBS-T \\
\hline rabbit anti p65 (C22B4) (\#4764) & $\begin{array}{l}\text { Cell Signalling/ New England } \\
\text { Biolabs, Frankfurt am Main GER }\end{array}$ & 1:1000 in $5 \%$ BSA in TBS-T \\
\hline $\begin{array}{l}\text { rabbit anti p-p70 S6 Kinase } \\
\text { (T389) (108D2) (\#9234) }\end{array}$ & $\begin{array}{l}\text { Cell Signalling/ New England } \\
\text { Biolabs, Frankfurt am Main GER }\end{array}$ & $1: 1000$ in $5 \%$ BSA in TBS-T \\
\hline $\begin{array}{l}\text { rabbit anti p70 S6 Kinase (49D7) } \\
(\# 2708)\end{array}$ & $\begin{array}{l}\text { Cell Signalling/ New England } \\
\text { Biolabs, Frankfurt am Main GER }\end{array}$ & 1:1000 in $5 \%$ BSA in TBS-T \\
\hline
\end{tabular}




\begin{tabular}{|c|c|c|}
\hline $\begin{array}{l}\text { rabbit anti p100/p52 (NF-KB2) } \\
\text { (\#4882) }\end{array}$ & $\begin{array}{l}\text { Cell Signalling/ New England } \\
\text { Biolabs, Frankfurt am Main GER }\end{array}$ & $1: 1000$ in $5 \%$ BSA in TBS-T \\
\hline $\begin{array}{l}\text { rabbit anti } \mathrm{p}-\mathrm{IKB} \alpha(\mathrm{S} 32)(14 \mathrm{D} 4) \\
(\# 2859)\end{array}$ & $\begin{array}{l}\text { Cell Signalling/ New England } \\
\text { Biolabs, Frankfurt am Main GER }\end{array}$ & $1: 1000$ in $5 \%$ BSA in TBS-T \\
\hline rabbit anti IkBa (44D4) (\#4812) & $\begin{array}{l}\text { Cell Signalling/ New England } \\
\text { Biolabs, Frankfurt am Main GER }\end{array}$ & $1: 1000$ in $5 \%$ BSA in TBS-T \\
\hline $\begin{array}{l}\text { rabbit anti p-SAPK/JNK } \\
\text { (T183/Y185) (81E11) (\#4668) }\end{array}$ & $\begin{array}{l}\text { Cell Signalling/ New England } \\
\text { Biolabs, Frankfurt am Main GER }\end{array}$ & 1:1000 in $5 \%$ BSA in TBS-T \\
\hline $\begin{array}{l}\text { rabbit anti JNK1/3 (C-17) } \\
\text { (\#sc-474) }\end{array}$ & Santa Cruz, Heidelberg GER & $1: 1000$ in $5 \%$ BSA in TBS-T \\
\hline rabbit anti RelB (C1E4) (\#4922) & $\begin{array}{l}\text { Cell Signalling/ New England } \\
\text { Biolabs, Frankfurt am Main GER }\end{array}$ & $1: 1000$ in $5 \%$ BSA in TBS-T \\
\hline $\begin{array}{l}\text { rabbit anti p-S6 ribosomal } \\
\text { protein }(\mathrm{S} 240 / 244)(\# 2215)\end{array}$ & $\begin{array}{l}\text { Cell Signalling/ New England } \\
\text { Biolabs, Frankfurt am Main GER }\end{array}$ & 1:1000 in $5 \%$ BSA in TBS-T \\
\hline $\begin{array}{l}\text { rabbit anti } 56 \text { ribosomal protein } \\
\text { (54D2) (\#2317) }\end{array}$ & $\begin{array}{l}\text { Cell Signalling/ New England } \\
\text { Biolabs, Frankfurt am Main GER }\end{array}$ & $1: 1000$ in $5 \%$ BSA in TBS-T \\
\hline $\begin{array}{l}\text { rabbit anti Tak1 (D94D7) } \\
(\# 5206)\end{array}$ & $\begin{array}{l}\text { Cell Signalling/ New England } \\
\text { Biolabs, Frankfurt am Main GER }\end{array}$ & $1: 1000$ in $5 \%$ BSA in TBS-T \\
\hline $\begin{array}{l}\text { goat anti mouse HRP polyclonal } \\
\text { (D1609) }\end{array}$ & Santa Cruz, Heidelberg GER & $1: 5000$ in $5 \%$ BSA in TBS-T \\
\hline $\begin{array}{l}\text { goat anti rabbit HRP polyclonal } \\
\text { (E1710) }\end{array}$ & Santa Cruz, Heidelberg GER & 1:5000 in $5 \%$ BSA in TBS-T \\
\hline
\end{tabular}




\subsection{Oligonucleotides}

Primers used for qRT-PCR are summarized in Table 2-8.

Table 2-8 Oligonucleotides used for qRT-PCR.

\begin{tabular}{|c|c|}
\hline Oligo & Sequence \\
\hline$A B L$ & $\begin{array}{l}\text { fwd: 5'-AGCCTGGCCTACAACAAGTTCTC-3' } \\
\text { rev: 5'-GACATGCCATAGGTAGCAATTTCC-3' }\end{array}$ \\
\hline B2M & $\begin{array}{l}\text { fwd: 5'-CTATCCAGCGTACTCCAAAGATTCA-3' } \\
\text { rev: 5'-TCTCTGCTGGATGACGTGAGTAAA-3' }\end{array}$ \\
\hline CCND2 & $\begin{array}{l}\text { fwd: 5'-CTGCGCAGGCAAGCACTAT-3' } \\
\text { rev: 5'-TCTTATCCTGCCAATTCAGTGTGA-3' }\end{array}$ \\
\hline CDK4 & $\begin{array}{l}\text { fwd: 5'-AGGCGACTGGAGGCTTTTG-3' } \\
\text { rev: 5'-GTGGCACAGACGTCCATCAG -3' }\end{array}$ \\
\hline CDKN1_P21 & $\begin{array}{l}\text { fwd: 5'-TGGAGACTCTCAGGGTCGAAA-3' } \\
\text { rev: 5'-GCGTTTGGAGTGGTAGAAATCTG-3' }\end{array}$ \\
\hline c-MYC & $\begin{array}{l}\text { fwd: 5'-AAGAGGGTCAAGTTGGACAGTTGC-3' } \\
\text { rev: 5'-TTTCGGTTGTTGCTGATCTGTCT-3' }\end{array}$ \\
\hline GAPDH & $\begin{array}{l}\text { fwd: 5'-ACCACTTTGTCAAGCTCATTTCCT-3' } \\
\text { rev: 5'-GTTGCTGTAGCCAAATTCGTTGT-3' }\end{array}$ \\
\hline hGLI1 & $\begin{array}{l}\text { fwd: 5'-CTGTGGGCAACATGAGTGTCTTAC-3' } \\
\text { rev: 5'-AATTCTGTTTCCCCAGGTAGGG-3' }\end{array}$ \\
\hline $\mathrm{hSHH}$ & $\begin{array}{l}\text { fwd: 5'-TTAAGGATGAAGAAAACACCGGAG-3' } \\
\text { rev: 5'-AGCGTTCAACTTGTCCTTACACCT-3' }\end{array}$ \\
\hline hSMO & $\begin{array}{l}\text { fwd: 5'-GTGACAAAGCCTCTGAAAGATGC-3' } \\
\text { rev: 5'-CCCACTAAATGGGTGTGAGGAA-3' }\end{array}$ \\
\hline PTCH1 & $\begin{array}{l}\text { fwd: 5'-TCGACATCAGCCAGTTGACTAAAC-3' } \\
\text { rev: 5'-TTAATGATGCCATCTGCATCCA-3' }\end{array}$ \\
\hline RNAPOLII & $\begin{array}{l}\text { fwd: 5'-CACTATTAAGAAGGCCAAGCAGGA-3' } \\
\text { rev: 5'-TGTTGTGTGCCTTCTCGATGAC-3' }\end{array}$ \\
\hline TAK1 & $\begin{array}{l}\text { fwd: 5'-ACAGCCTATTCCAAGCCTAAACG-3' } \\
\text { rev: 5'-CAGAATGTTGCCAAATGAAGCA-3' }\end{array}$ \\
\hline
\end{tabular}




\begin{tabular}{|l|l|}
\hline ICAM1 & $\begin{array}{l}\text { fwd: 5'-TTCACAATGACACTCAGCGGTC-3' } \\
\text { rev: 5'-AGTGCAAGCTCCCAGTGAAATG-3' }\end{array}$ \\
\hline ANK3 & $\begin{array}{l}\text { fwd: 5'-CGTAACAGCGAACGGTCAGTC-3' } \\
\text { rev: 5'-TACTTCCTGCTGACATTTCTTCCAC-3' }\end{array}$ \\
\hline SIRPA & $\begin{array}{l}\text { fwd: 5'-CTTCTTCTACAAGGTTGCATGAGCC-3' } \\
\text { rev:5'-GTCTGAATGCTGGCATACTCCG-3' }\end{array}$ \\
\hline RFTN1 & $\begin{array}{l}\text { fwd: 5'-GAGTGTATCCACCAAGCAGATTGT C-3' } \\
\text { rev: 5'-CCTCATCTGCCTGTTGTGCAT-3' }\end{array}$ \\
\hline LINC00158 & $\begin{array}{l}\text { fwd: 5'-GAACCCTGAGCAGAACACATGC-3' } \\
\text { rev: 5'-GACGACCGCTTTCTTAAACTTTCTC-3' }\end{array}$ \\
\hline CTDSPL & $\begin{array}{l}\text { fwd: 5'-TATTGTTCCGGTTGAAATCGATG-3' } \\
\text { rev: 5'-GGCCAAGCTGGCAGTAAAGAG-3' }\end{array}$ \\
\hline
\end{tabular}

\subsection{Ready to Use Reaction Systems}

In Table 2-9 the Ready to use reaction systems applied during this study are listed.

Table 2-9 Reaction Systems

\begin{tabular}{|l|l|}
\hline Description & Manufacturer \\
\hline RNA Isolation Kit & Machery+Nagel, Düren GER \\
\hline Rneasy ${ }^{\circledast}$ Mini Kit & Qiagen, Hilden GER \\
\hline SuperscriptIITM RT Kit & Invitrogen, Karlsruhe GER \\
\hline SignalBoostTM Immunoreaction Enhancer Kit & Calbiochem via Merck, Darmstadt GER \\
\hline
\end{tabular}




\subsection{Software used}

If not stated elsewhere the following software products were used: the present thesis was written in Microsoft ${ }^{\circledR}$ Office Word 2013 and 2007. Literature-management and bibliography was generated using Mendeley Desktop Version 1.8.4. Graphs were created using GraphPad PRISM 5.04 and figures were assembled using Adobe ${ }^{\circledR}$ Photoshop $^{\circledR}$ CS2 and Microsoft ${ }^{\circledR}$ Office PowerPoint 2013 and 2007. Gene expression data were manually compared and analyzed using VENNY (Oliveros, 2007).

\subsection{Cell Biology}

\subsubsection{Cell Culture Techniques}

Burkitt Lymphoma cell lines were cultured in RPMI-1640, $10 \%$ (v/v) FCS, $200 \mathrm{U} / \mathrm{ml}$ Penicilin, $200 \mu \mathrm{g} / \mathrm{ml}$ Streptomycin, $20 \mathrm{nM}$ BCS, $50 \mu \mathrm{M} \alpha$-Thioglycerol, $1 \mathrm{mM}$ Sodium pyruvate. Cells were cultured at $37^{\circ} \mathrm{C}, 5 \% \mathrm{CO}_{2}$ and splitted daily depending on the observed cell proliferation (cell density: $0.3 \times 10^{6}$ cells $/ \mathrm{ml}-1 \times 10^{6}$ cells $/ \mathrm{ml}$ ). The day before the experiment $0.3 \times 10^{6}$ cells $/ \mathrm{ml}$ were seeded.

DLBCL cell lines, except OCI-Ly1, OCl-Ly3 and OCI-Ly7, were cultured in RPMI-1640, $10 \%$ (v/v) FCS, $200 \mathrm{U} / \mathrm{ml}$ Penicilin, $200 \mathrm{\mu g} / \mathrm{ml}$ Streptomycin and kept in a cell density of $0.5 \times 10^{6}-1 \times 10^{6}$ cells $/ \mathrm{ml}$. OClLy1 and OCI-Ly7 were cultured in IMDM, 10 \% (v/v) FCS, $200 \mathrm{U} / \mathrm{ml}$ Penicilin, 200 mg/ml Streptomycin,

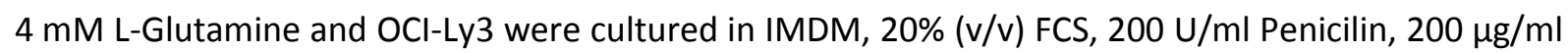
Streptomycin, 4 mM L-Glutamine.

Cells were counted in $0.4 \%$ Trypan blue using a Neubauer counting chamber. To cultivate cells from a frozen aliquot, thawed cells were washed with $10 \mathrm{ml}$ of the respective cell culture medium (centrifugation $250 \times \mathrm{g}, 7 \mathrm{~min}$ at RT). The cell pellet was resuspended in 5-10 ml medium and cells were incubated at $37^{\circ} \mathrm{C}, 5 \% \mathrm{CO}_{2}$. To freeze cells approximately $1 \times 10^{7}$ cells were sedimented as described above and resuspended in $1 \mathrm{ml}$ freezing medium (90 \% FCS, 10 \% DMSO). Cells were frozen in cryo boxes containing isopropanol to obtain a constant cooling of approximately $1^{\circ} \mathrm{C} / \mathrm{min}$ and stored immediately at $-80^{\circ} \mathrm{C}$ for 24 hours before permanent storage at $-150^{\circ} \mathrm{C}$.

\subsubsection{Activation of B cells with Soluble Stimulating Factors}

Burkitt lymphoma cell lines were seeded in fresh media in a density of $3 \times 10^{5}$ cells $/ \mathrm{ml}$ (DLBCLs: $5 \times 10^{5}$ cells $/ \mathrm{ml}$ ) the day prior to experimental handling. In order to stimulate the cells, they were counted, centrifuged at $250 \times \mathrm{g}$ for $7 \mathrm{~min}$, resuspended in fresh corresponding medium supplied with $10 \mathrm{mM}$ Hepes $\left(1 \times 10^{6}\right.$ cells $\left./ \mathrm{ml}\right)$ and supplemented with sCD40L $(200 \mathrm{ng} / \mathrm{ml})$ or anti human $\mathrm{IgM} \mathrm{F}(\mathrm{ab})_{2}$ fragment $(13 \mu \mathrm{g} / \mathrm{ml})$. Cells were incubated at $37^{\circ} \mathrm{C}, 5 \% \mathrm{CO}_{2}$ for $15 \mathrm{~min}, 30 \mathrm{~min}, 60 \mathrm{~min}, 90 \mathrm{~min}$, $120 \mathrm{~min}, 150 \mathrm{~min}, 180 \mathrm{~min}, 210 \mathrm{~min}, 240 \mathrm{~min}, 360 \mathrm{~min}, 480 \mathrm{~min}$ and 24 hours for microarray analyses. Cells were harvested $\left(10 \mathrm{~min}, 300 \times \mathrm{g}, 4^{\circ} \mathrm{C}\right)$ and washed with PBS containing $100 \mathrm{mM}$ sodium orthovanadate. Samples were frozen at $-80^{\circ} \mathrm{C}$ and RNA was isolated with the RNeasy Kit 
(Qiagen) for microarray analyses or with the NucleoSpin ${ }^{\circledast}$ RNA II Kit (Macherey-Nagel, Düren Germany).

\subsubsection{Inhibitor Treatment}

16 hours prior to inhibitor treatment cells were seeded in serum-reduced media $(0.5 \%$ FCS $)$ at $3 \times 10^{5}$ cells $/ \mathrm{ml}$ (BL cell lines) or at $5 \times 10^{5}$ cells $/ \mathrm{ml}$ (DLBCL cell lines). Before inhibitor treatment, cells were counted, centrifuged $\left(250 \times \mathrm{g}, 7 \mathrm{~min}\right.$ ) and seeded in a density of $1 \times 10^{6}$ cells $/ \mathrm{ml}$ in fresh serumreduced media containing $25 \mathrm{mM}$ Hepes. For microarray analyses, cells were treated with $100 \mathrm{nM}$ (5Z)-7-Oxozeaenol (TAK1/MAP3K7 inhibitor), $7 \mu \mathrm{M}$ IKK2-VIII inhibitor, $10 \mu \mathrm{M}$ Ly294002 (PI3K inhibitor), $2 \mu$ M SB203580 (p38/MAPK14 inhibitor), $10 \mu \mathrm{M}$ SP600125 (JNK inhibitor) or $10 \mu \mathrm{M}$ U0126 (MEK1/2 inhibitor) for $3 \mathrm{~h}$ prior to BCR or CD40 activation. The stimulation was performed for 3 hours to monitor gene expression changes, or for 30 minutes to validate pathway activation via WB respectively. Cells were harvested at $300 \times \mathrm{g}$ at $4^{\circ} \mathrm{C}$ for $10 \mathrm{~min}$ and resuspended in ice-cold PBS, supplemented with $1 \mathrm{mM}$ sodium orthovanadate. Dry pellets were frozen at $-20^{\circ} \mathrm{C}$ for protein analyses or $-80^{\circ} \mathrm{C}$ for RNA processing.

\subsubsection{Transfection of siRNA}

Small-interfering RNA (siRNA) is a short (20-25bp) double-stranded RNA molecule, which can be theoretically used to knock down any gene of interest when synthetically engineered. siRNA can be introduced in mammalian cells by transfection. Transfection can be carried out using calcium phosphate by mixing a cationic lipid with the siRNA to produce liposomes, which fuses with the cell membrane. Another transfection method is electroporation. For electroporation, the cells are exposed to short pulses of an intense electric field, which transiently increases the permeability of the cell membrane.

To achieve a knock down of Tak1, corresponding siRNAs (Silencer ${ }^{\circledR}$ Select, Invitrogen, Darmstadt GER) were transfected into Burkitt's and Diffuse large B cell lymphoma cell lines using the GenePulser Xcell (Bio-Rad, Munich GER). $2 \mu \mathrm{g}$ of corresponding siRNA were transfected by electroporation $(300 \mathrm{~V}, 150 \mu \mathrm{F}, 1540 \Omega)$ in $2.5 \times 10^{6}$ cells $/ \mathrm{ml}$ lymphoma cell lines in $200 \mu$ l Opti-MEM ${ }^{\circledR}$ (Invitrogen, Darmstadt GER) and transferred into $5 \mathrm{ml}$ pre-warmed RPMI 1640, supplemented with $10 \%(\mathrm{v} / \mathrm{v})$ FCS, $200 \mathrm{U} / \mathrm{ml}$ Penicilin, $200 \mu \mathrm{g} / \mathrm{ml}$ Streptomycin, $20 \mathrm{nM} \mathrm{BCS}, 50 \mu \mathrm{M} \alpha$-Thioglycerol, $1 \mathrm{mM}$ Sodium pyruvate and $25 \mathrm{mM}$ Hepes. Transfected cells were incubated for 24 hours at $37^{\circ} \mathrm{C}, 5 \% \mathrm{CO}_{2}$ and subsequently transfected a second time. A non-sense siRNA without a human target was used as control (scrbl). Upon two transfections and subsequent 24 hours of incubation, control-transfected cells were subjected to inhibitor treatment and stimulation with SCD40L $(200 \mathrm{ng} / \mathrm{ml})$ or $\alpha$-lgM $(13 \mu \mathrm{g} / \mathrm{ml})$ 


\subsection{Protein Biochemistry}

\subsubsection{Preparation of Cell Lysates}

Cells were harvested using sedimentation $\left(300 \times \mathrm{g}, 7 \mathrm{~min}, 4^{\circ} \mathrm{C}\right)$ and washed once with PBS containing $1 \mathrm{mM}$ Sodium orthovanadate. Dry pellets were stored at $-20^{\circ} \mathrm{C}$ until further preparation.

\section{Whole cell lysates}

Whole cell lysates were prepared by resuspending a cell pellet corresponding to $1 \times 10^{6}$ cells in $50 \mu \mathrm{l}$ RIPA buffer. The cell suspension was incubated for 30 min on ice, subsequently residual cell debris was sedimented at $13000 \mathrm{rpm}$ for $15 \mathrm{~min}$ at $4^{\circ} \mathrm{C}$. The supernatant, containing the whole cell lysate, was transferred into a fresh tube and protein concentration was determined using the RotiQuant ${ }^{\circledR}$ assay (Roth, Karslruhe GER). Protein concentration was measured with a microplate reader (Infinite ${ }^{\circledR}$ F50, Tecan, Männedorf $\mathrm{CH}$ ).

\section{Cell fractionation}

To demonstrate activation of the NF-kB pathway, nuclear translocation of NF-kB family members was monitored. Therefore, separation of cytosolic protein from nuclear protein was necessary. To obtain nuclear extracts from freshly harvested cell pellets, cell fractionation according to Schreiber et al. (Schreiber et al., 1989) was accomplished. Therefore $1 \times 10^{7}$ cells were harvested $\left(300 \times \mathrm{g}, 10 \mathrm{~min}, 4^{\circ} \mathrm{C}\right)$ and washed twice with PBS containing $1 \mathrm{mM}$ Sodium orthovanadate. Pellets were lysed in $400 \mu \mathrm{l}$ buffer A and incubated 15 min on ice. $25 \mu \mathrm{l} 10 \%$ NP-40 were added and vigorously vortexed for $10 \mathrm{~s}$. Samples were sedimented for $5 \mathrm{~min}, 4^{\circ} \mathrm{C}$ at $13000 \mathrm{rpm}$ and the supernatant containing cytosolic protein was transferred to a new tube. The pellet was covered with $50 \mu$ buffer B and shaked strongly for $25 \mathrm{~min}$ at $4^{\circ} \mathrm{C}$ in the cold room. Upon sedimentation for $5 \mathrm{~min}$ at $13000 \mathrm{rpm}, 4^{\circ} \mathrm{C}$, the supernatant containing the nuclear proteins was transferred into a new tube. Protein concentration was determined using the RotiQuant ${ }^{\circledR}$ assay (Roth, Karslruhe GER).

\subsubsection{SDS-PAGE and Western Blot}

For separation of proteins depending on their molecular weight sodium dodecyl sulphate polyacrylamide gelelectrophoresis (SDS-PAGE) was used (Ahn et al., 2001; Laemmli, 1970). Using the BioRad System (Munich, GER), polyacrylamide gels comprised of a $10 \%$ separation gel and a $5 \%$ stacking gel were prepared. $20-30 \mu \mathrm{g}$ of protein samples were loaded with $4 x$ RotiLoad $^{\circledR}$-loading dye, denaturized at $95^{\circ} \mathrm{C}$ for $5 \mathrm{~min}$ and loaded onto the gel. For protein size determination, RainbowFullRange $^{\mathrm{TM}}$ marker was loaded additionally. Gels were run with $20 \mathrm{~mA}$ per gel for 1 hour and subsequently proteins were transferred to a nitrocellulose membrane (Hybond ${ }^{\mathrm{TM}}-\mathrm{C}$ extra, GE Healthcare, Munich, Germany) using the Tank Blot System (BioRad, Munich, Germany). The separating gel was cut from the stacking gel and equilibrated for one minute in Transfer Buffer. For 
protein transfer a pile was carefully prepared as follows: cathode (-), foam pad, 2 Whatman ${ }^{\mathrm{TM}}$-papers, separating gel, nitrocellulose membrane, 2 Whatman ${ }^{\mathrm{TM}}$-papers, foam pad, anode $(+)$. Transfer was conducted at $100 \mathrm{~V}$ for 1 hour at $4^{\circ} \mathrm{C}$.

\subsection{Molecular Biology}

\subsection{1 mRNA Isolation}

mRNA was isolated from cell pellets using the RNeasy ${ }^{\circledR}$ Mini Kit (Qiagen, Hilden, Germany) or NucleoSpin ${ }^{\circledast}$ RNA Kit (Macherey\&Nagel, Düren, Germany) according to manufacturer instructions. mRNA was eluted using $40 \mu \mathrm{l}$ RNAse-free water. Concentrations were measured by NanoDrop ${ }^{\mathrm{TM}}$ measurement.

\subsubsection{Reverse Transcription}

In order to obtain CDNA, 1-2 $\mu$ g mRNA was reversely transcribed using the SuperScriptIIM RT Kit (Invitrogen, Darmstadt GER). Reverse transcription was initiated with random hexamer primer. $2 \mu$ l random hexamer primer $(100 \mu \mathrm{M})$ and 1 - $2 \mu \mathrm{g}$ mRNA in $10 \mu \mathrm{l} \mathrm{H}_{2} \mathrm{O}$ were added to each cup. The mix was denaturated for $10 \mathrm{~min}$ at $70^{\circ} \mathrm{C}$ in a thermocycler (Biometra). Subsequently $8 \mu \mathrm{l}$ of Master Mix (Table 2-10) was added and the complete mixture was incubated in a thermocycler with the following program (Table 2-10).

Table 2-10 Master Mix and thermocycler program for reverse transcription of mRNA

\begin{tabular}{|r|r|}
\hline Master Mix & thermocycler program \\
\hline $4 \mu \mathrm{l} 5 \mathrm{x}$ First Strand Buffer & \\
\hline $2 \mu \mathrm{l}$ DTT $(0.1 \mathrm{M})$ & $42^{\circ} \mathrm{C} 60 \mathrm{~min}$ \\
\hline $1 \mu \mathrm{l}$ dNTPs (10 mM each) & $65^{\circ} \mathrm{C} 10 \mathrm{~min}$ \\
\hline $1 \mu$ l SuperScript RT II & $4^{\circ} \mathrm{C} \infty$ \\
\hline
\end{tabular}

\subsection{3 qRT-PCR (quantitative Real Time - Polymerase Chain Reaction)}

For the quantification of transcripts, a SYBR ${ }^{\circledR}$ Green based system was used. The cyanine dye SYBR ${ }^{\circledR}$ Green intercalates in double-stranded DNA. The resulting DNA-dye-complex absorbs blue light $\left(\lambda_{\max }=488 \mathrm{~nm}\right)$ and emits green light $\left(\lambda_{\max }=522 \mathrm{~nm}\right)$ and can therefore be used to quantify doublestranded PCR products over time during a PCR reaction. The number of PCR cycles at which a certain threshold of fluorescence intensity is achieved ( $\mathrm{Ct}$ - value, cycle threshold), directly correlates to the number of DNA templates present in the reaction. 
Transcript quantification was performed with Fast SYBR ${ }^{\circledR}$ Green (Applied Biosystems, Foster City USA) for GC B samples and a self-made SYBR Green Mix (see section Materials) for all samples obtained from cell lines. To carry out the PCR reaction and fluorescence detection an ABI PRISM 7900HT sequence detection system (Applied Biosystems, Foster City USA) was used (SDS 2.4). For GC B samples, cDNA corresponding to $2.5 \mu \mathrm{g}$ RNA was used as template. For cDNA obtained from cell line experiments a corresponding amount of 10 - $20 \mu \mathrm{g}$ RNA was used. PCR reactions were performed in a 384-well clear optical plate. The relative mRNA expression levels were determined by normalizing the mean Ct-values of triplets to the $\mathrm{Ct}$ values of a housekeeping gene:

$$
\Delta \mathrm{Ct}=\mathrm{Ct}_{\text {gene of interest }}-\mathrm{Ct}_{\text {housekeeping gene }}
$$

The $\Delta \mathrm{Ct}$ values of the modified sample and the control sample can then be compared by calculating $\Delta \Delta \mathrm{Ct}$ :

$$
\Delta \Delta \mathrm{Ct}=\Delta \mathrm{Ct}_{\text {modified sample }}-\Delta \mathrm{Ct}_{\text {control }}
$$

$\Delta \Delta \mathrm{Ct}$ is a relative measurement, giving the values for the differences of cycles of the amplicon of the gene of interest after treatment in comparison to control. Assuming that the efficiency for both genes (household gene and gene of interest) is $100 \%$, one can calculate the $n$-fold change $=2^{-\Delta \Delta C t}$. As housekeeping gene, $A B L$ was chosen in this study. All primers for the qRT-PCR reactions were designed with the use of PrimerExpress ${ }^{\mathrm{TM}}$ software (Applied Biosystems, Foster City USA) on the basis of the published NCBI mRNA sequence (http://www.ncbi.nlm.nih.gov/sites/entrez/). Specificity was tested with the help of BLAST software (http://www.ncbi.nlm.nih.gov/blast/Blast.cgi). In addition, the efficiency of each pair of primers was assessed, running a qRT-PCR on a dilution series of template DNA and calculating the slope $m$ of the linear regression of the standard curve. The efficiency was calculated by

$$
E=10^{-1 / m}-1 .
$$

Thus, an efficiency of $100 \%$ results in a slope of -3.32 which corresponds to a doubling of the amount of amplicon with one cycle. Primer pairs with an efficiency lower than $93 \%$ were abolished. Analyses were accomplished using Microsoft ${ }^{\circledR}$ Office Excel 2007.

\subsection{Microarray Analyses}

Two different types of Affymetrix GeneChip ${ }^{\circledR}$ microarray platforms were used in the present study. For pathway perturbation analyses via inhibitor use (chapter 3.3), the Affymetrix GeneChip Human Genome U133 Plus 2.0 array was used to ensure comparability to former derived data sets. The time course analyses were hybridized on Affymetrix Human Gene 1.0 ST microarrays. In contrast to the classical 3' gene expression arrays, the Human Gene 1.0 ST microarray platform is a whole-transcript 
expression array, including approx. 26 probes per annotated gene, spread across the whole length of the gene.

For both microarray platforms, cDNA was synthesized using $1 \mu \mathrm{g}$ corresponding RNA and including Poly-A RNA controls with the help of the Affymetrix GeneChip ${ }^{\circledR}$ Poly-A RNA control Kit. Secondly, cRNA synthesis and cleanup was carried out using the Affymetrix GeneChip ${ }^{\circledR}$ IVT Labelling Kit. Subsequently, fragmentation and labeling for microarray hybridization was accomplished with the corresponding kits according to manufacturer's instructions. The whole process, including final hybridization, washing, staining and scanning was executed in the DNA Micorarray and DeepSequencing Facility of the University Medical Center Göttingen (UMG) with the help of Dr. G. SalinasRiester and S. Luthin. Raw data were transferred to the Institute of Functional Genomics, University Regensburg, and statistically analyzed by K. Meyer, M. Pirkl and Prof. Dr. R. Spang.

Microarray data preprocessing - Data analysis was performed using the statistical computing environment $\mathrm{R}$ and the life-science related extension Bioconductor (http://www.bioconductor.org). Gene expression profiles were background corrected and normalized on probe level using a variance stabilization method (Huber et al., 2002). Normalized probe intensities were summarized into gene expression levels using an additive model described in (Irizarry, 2003) and fitted by a median polish routine. Additionally, batch effects were corrected using ComBat (Johnson et al., 2007) by estimating additive and multiplicative effects for each batch and normalizing accordingly.

Differential gene expression - An additive linear model approach (Smyth, 2005) was used to calculate effects of experimental conditions on each probe set. The experiments with single inhibitors/stimulations are used to calculate the individual effect. Synergetic effects are then inferred from the combinatorial experiments.

Nested Effects Models - The effects of gene perturbation via inhibitor use and subsequent stimulation on the whole genome gene expression level were analyzed using a class of probabilistic models called 'Nested Effects Models' (NEMs) (Fröhlich et al., 2007; Markowetz et al., 2005).

GO KEGG and GSEA - Gene set enrichment analysis (GSEA) of ranked gene lists was performed using the Java implementation of GSEA obtained from http://www.broadinstitute.org/gsea/. GSEA was conducted in the mode for pre-ranked gene lists on the C2 set of curated gene signatures from the Molecular Signature Database (MSigDB). GO and KEGG pathway enrichment analyses were performed using the gokeggLister method implemented in the package compdiagTools. 


\section{Results}

\subsection{Temporal pattern of whole genome gene expression changes upon signaling pathway activation}

In the last decade, global gene expression analyses have been excessively used for developing strategies to describe and stratify lymphoma cases. Hence, a molecular definition of BL was identified, distinguishing mBL from non-mBL, including DLBCLs (Dave et al., 2006; Hummel et al., 2006). However, it remains unclear to which extent distinct signaling pathways of the immune response are in charge of the differences in gene expression that distinguish lymphoma. In former analyses in the group of D. Kube, BL cells were stimulated with factors known to be involved in B cell signaling, microenvironment and lymphoma pathogenesis and subjected to microarray analysis. Thereby, distinct in vitro stimuli could characterize individual NHL and signaling pathways dominantly involved in these global gene expression patterns have been identified (Schrader et al., 2012b). In the present study, two of the stimuli inducing the strongest gene expression changes (CD40 and B cell receptor activation) were used in a time-resolved analysis to provide data allowing the prediction of regulatory feedback loops of certain oncogenic pathways.

In order to obtain a defined time course of pathway perturbation at the global level, we used human transformed GC B cells as a model system in vitro (BL2). Stimulation of cells in vitro was done in triplicates by adding $\alpha-\operatorname{IgM~} F(a b)_{2}$-fragment to activate $B C R$ signaling or SCD40L to activate $C D 40$ mediated signaling, respectively. Samples were collected in defined time intervals until $480 \mathrm{~min}$ (Figure 3-1 A). The corresponding RNA samples were hybridized to Human Gene 1.0 ST arrays (Affymetrix, Santa Clara). Respective bioinformatical and high-dimensional statistical data analysis was performed in parallel by several statisticians belonging to the HaematoSys-consortium. Data preparation (background correction, normalization, estimation of gene expression levels by a median polish routine: Katharina Meyer, Regensburg) exhibited a unique gene expression pattern (Figure 3-1 $B+D)$. Upon $\alpha$-IgM stimulation 2509 genes with fold change $\geq 3$ were altered in their expression level, while CD40 activation led to change of expression level in 2447 genes (here fold change $\geq 1.5$ was chosen, since the overall variance of expression changes was smaller than in $\alpha$-IgM stimulated cells).

In parallel, CD40 and BCR mediated signaling was monitored on the protein level by detecting changes in protein phosphorylation and degradation (Figure 3-1 C +E). To describe the effects and their time courses, activation of the following pathways was monitored by Western Blot analyses: MAPK signaling (phosphorylation of Erk and p38), NF-KB signaling (via the degradation of IKB $\alpha$ ) and PI3K/Akt signaling (phosphorylation of Akt). 
A

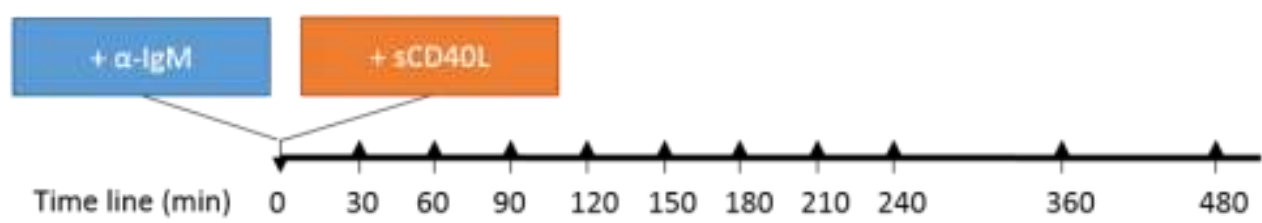

B

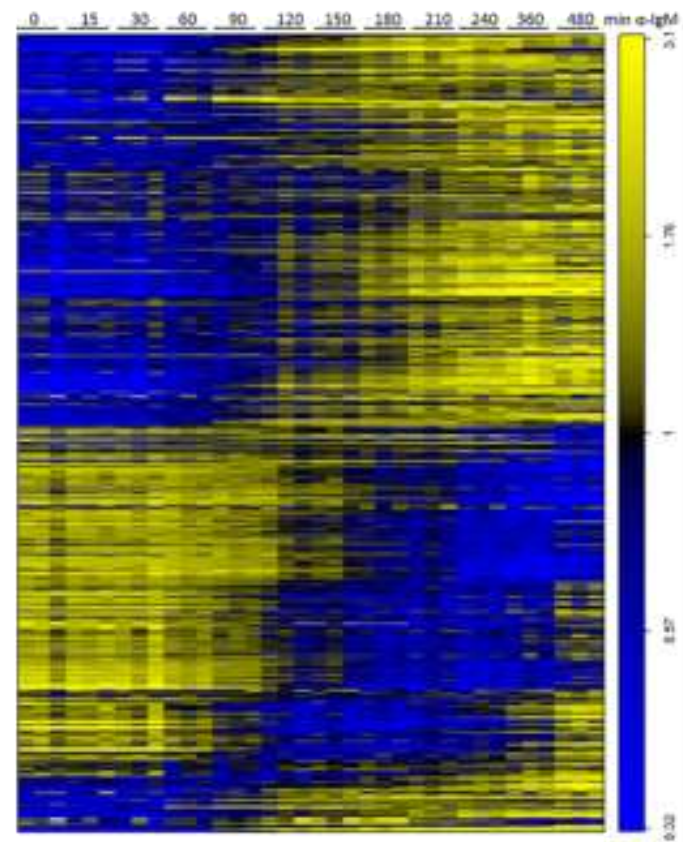

$\mathrm{C}$

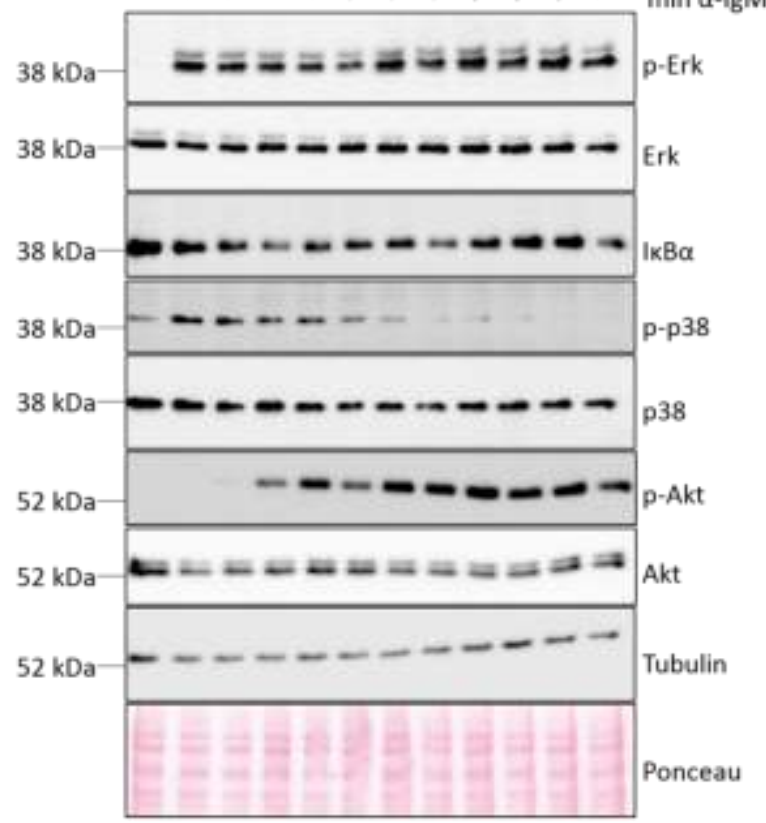

D

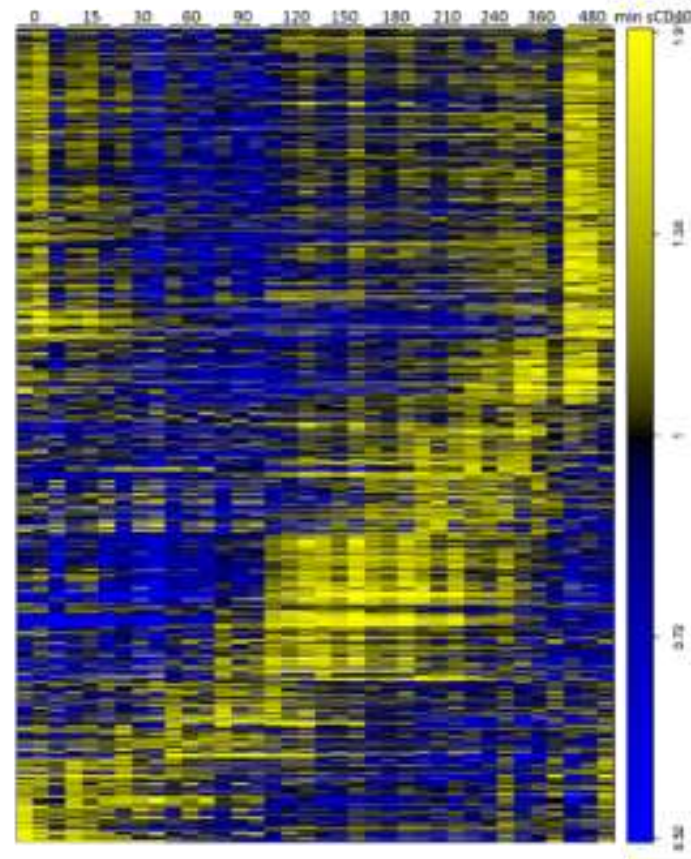

○

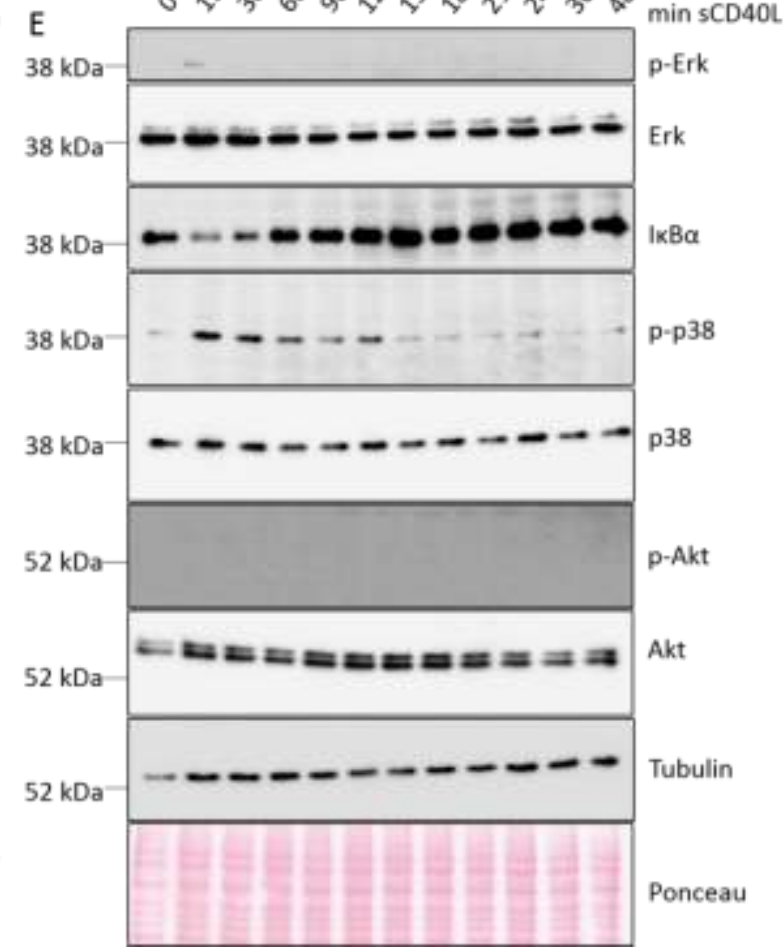

Figure 3-1 Time course analyses of gene expression changes on the whole genome level upon stimulation of human transformed germinal center B cells. A BL2 cells were stimulated with $200 \mathrm{ng} / \mathrm{ml} \mathrm{sCD} 40 \mathrm{~L} \mathrm{or} 13 \mu \mathrm{g} / \mathrm{ml}$ anti-IgM $\mathrm{F}(\mathrm{ab})_{2}$ fragment respectively and samples were harvested every 30 minutes for up to 4 hours, plus additional later time points at 6 and 8 hours, $\mathbf{B}+\mathbf{D}$ Heatmaps displaying gene expression changes on the whole genome level. Each row represents one of the TOP100 most highly responding genes. Each column represents 
one sample. Three biological replicates of each sample either with BCR (B) or CD40 activation (D) were hybridized to HU Gene 1.0 (Affymetrix). Additionally, changes in protein phosphorylation and degradation were documented by Western Blot analyses $(\mathbf{C}+\mathbf{E})$.

As shown in Figure 3-1 C, $\alpha$-IgM stimulation of $B L$ cells induced a rapid and sustained phosphorylation of Erk as well as an equally rapid activation of p38 (p-p38). However, the phosphorylation status of p38 declined 90 min after BCR crosslink and reached basal level upon 150 min after stimulation. CD40 activation of BL cells showed a similar phosphorylation course of p38, although an activation of MAPK signaling through phosphorylation of Erk was hardly detectable upon 15 min after CD40 stimulation, but not at any other time point after stimulation (Figure 3-1 E). The activation of NF-KB signaling was detected by monitoring the degradation of the NF-KB inhibitor IKB $\alpha$. CD40L strongly activated canonical NF-KB signaling rapidly after stimulation (15 $\mathrm{min}-30 \mathrm{~min}$ ), whereas $\alpha$-lgM stimulation of BL cells resulted only in a marginal degradation of IKB $\alpha$ at a later time point (60 min after BCR activation). In contrast, BCR crosslink led to a pronounced activation of the PI3K/Akt pathway (p-Akt, Figure 3-1 C), whereas upon CD40L stimulation a phosphorylation of Akt was not detectable. Thus, we confirm that $\alpha$-IgM and CD4OL treatment activate, although with distinct kinetics, partially overlapping pathways.

\subsection{1 $\alpha$-IgM stimulation of $B L$ cells changes the $m B L$ index towards non-mBLs}

To further prove the functional relevance of $\mathrm{CD} 40 \mathrm{~L}$ and $\alpha$-IgM stimulated $\mathrm{BL}$ cells in vitro, it was investigated how the activation of the $B L$ cells influences their $\mathrm{mBL}$ index. The $\mathrm{mBL}$ index value summarizes the expression of the $58 \mathrm{mBL}$ signature genes and represents a measure for 'Burkittlikeness' (Hummel et al., 2006). Designated mBL and non-mBL patient samples from the MMML study were hybridizied to HU Gene 1.0 (Affymetrix, Santa Clara, US) to achieve comparability to the time course data. Subsequently, the expression of the $58 \mathrm{mBL}$ signature genes in the experimental time course samples was determined and each sample was assigned an mBL-signature index score, with a higher score reflecting a higher similarity of gene expression in comparison to the $\mathrm{mBL}$ core samples (Hummel et al., 2006). One can observe, as expected, that untreated BL2 cells showed a similar mBL index as proven BL NHL cases (Figure 3-2). Sustained CD40L stimulation for up to 8 hours reduced the $\mathrm{mBL}$ index score slightly, but did not change the expression of the $\mathrm{mBL}$ signature genes in an intense manner. In contrast, anti-IgM stimulation of BL2 cells for up to 8 hours led to a more intense reduction in the $\mathrm{mBL}$ index score over time. One could conclude that the stimulation of BL2 cells with $\alpha$-IgM $F(a b)_{2}$ fragments shifts the gene expression profile of the former $\mathrm{mBL}$ cells towards a gene expression profile of DLBCLs. Thus, signaling pathways, which are activated in consequence of $B$ cell receptor cross linking, seem to induce gene expression changes, which in turn are relevant for a certain "DLBCL-likeness". To further illuminate the substantial differences, which characterize DLBCL 
and $\mathrm{BL}$, their clinical manifestations and inevitable therapies, a closer view into BCR mediated signaling events in transformed germinal center B cells will be very helpful.
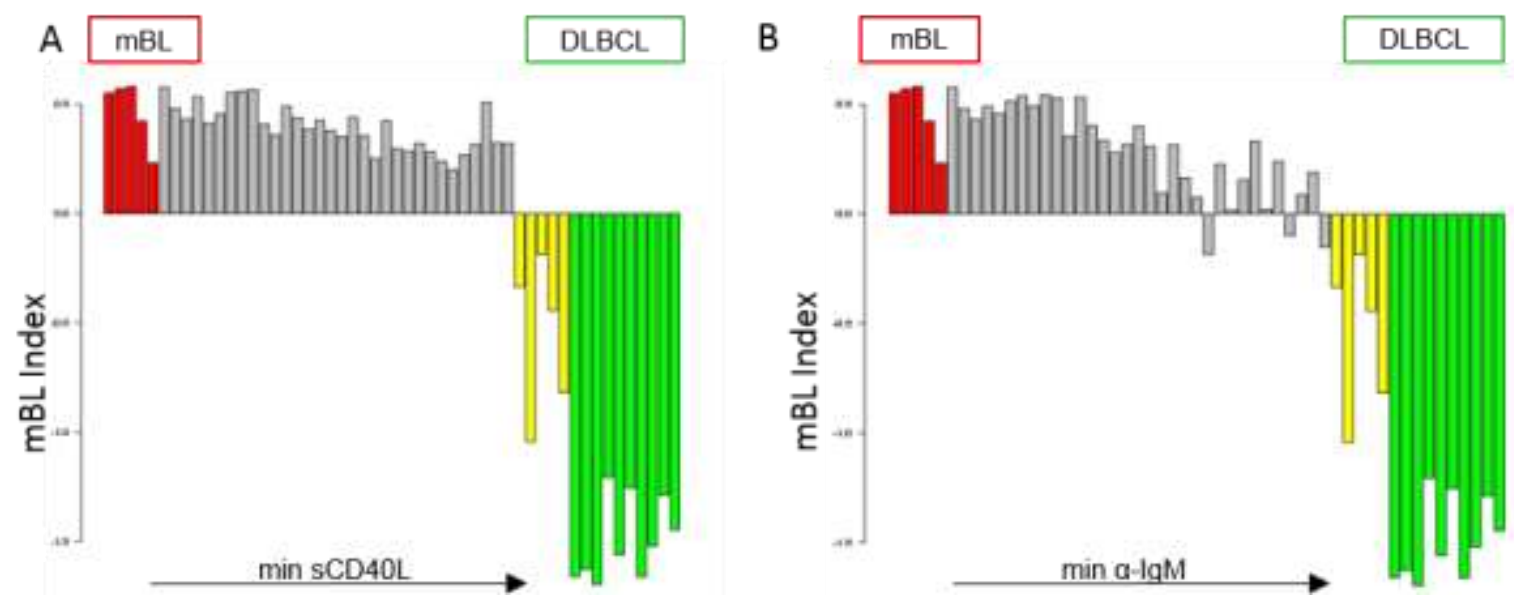

Figure 3-2 Sustained BCR activation in BL cells shifts $\mathrm{mBL}$ index towards the index of non-mBLs. $\mathrm{mBL}$ index (Hummel et al., 2006) of time course microarray data (grey bars) upon (A) CD40L stimulation and (B) BCR crosslink was calculated and compared to defined $\mathrm{mBL}$ (red bars), intermediate (yellow bars) and non-mBL cases (green bars, DLBCL). Each bar represents one sample hybridized to HU Gene 1.0 (Affymetrix).

\subsubsection{Causality Network of BCR induced gene expression correlations}

A time course of signaling events is especially interesting to analyze in order to find interdependent groups of genes. Data obtained in the present study were taken for network modeling to display correlations and causalities between subsets of genes. Normalized and calibrated raw data were taken and data were subsequently pre-filtered in two independent steps to exclude genes, which (step 1) can be assigned to noise and which (step 2) don't show any changes in expression over time. Noise-cancellation was accomplished assuming a Gaussian distribution of the expression values and modeling the overall signal through heuristic fitting of a two-component-mixture. Thereby, noise was cancelled and the signal remained. For anti-IgM stimulated data 8012 out of 21995 genes represented on the array remained. Secondly, genes which don't change their expression level with time elapsed, were excluded. Therefore, Fisher's g-test, testing on white noise of unknown frequency was used (Wichert et al., 2003). Using a False-Discovery-Rate of 0.1, 2832 genes remained, significantly changing their expression upon BCR crosslink. Using Bayesian Hierarchical Clustering (Cooke et al., 2011; Heller and Ghahramani, 2005; Savage et al., 2009) clustering of genes with a very similar time course was computed. This method reduced the dimensions to 22 clusters of genes. Subsequently, clusters with marginal changes over time were observed. All clusters of genes with an expression range less than 0.5 and less than $10 \%$ highly significant genes (FDR $<0.05)$ were excluded. Finally based on an algorithm calculating partial correlation between the clusters (SIMoNe (Charbonnier et al., 2010)) a network displaying causal relationships was modeled (Figure 3-3). 


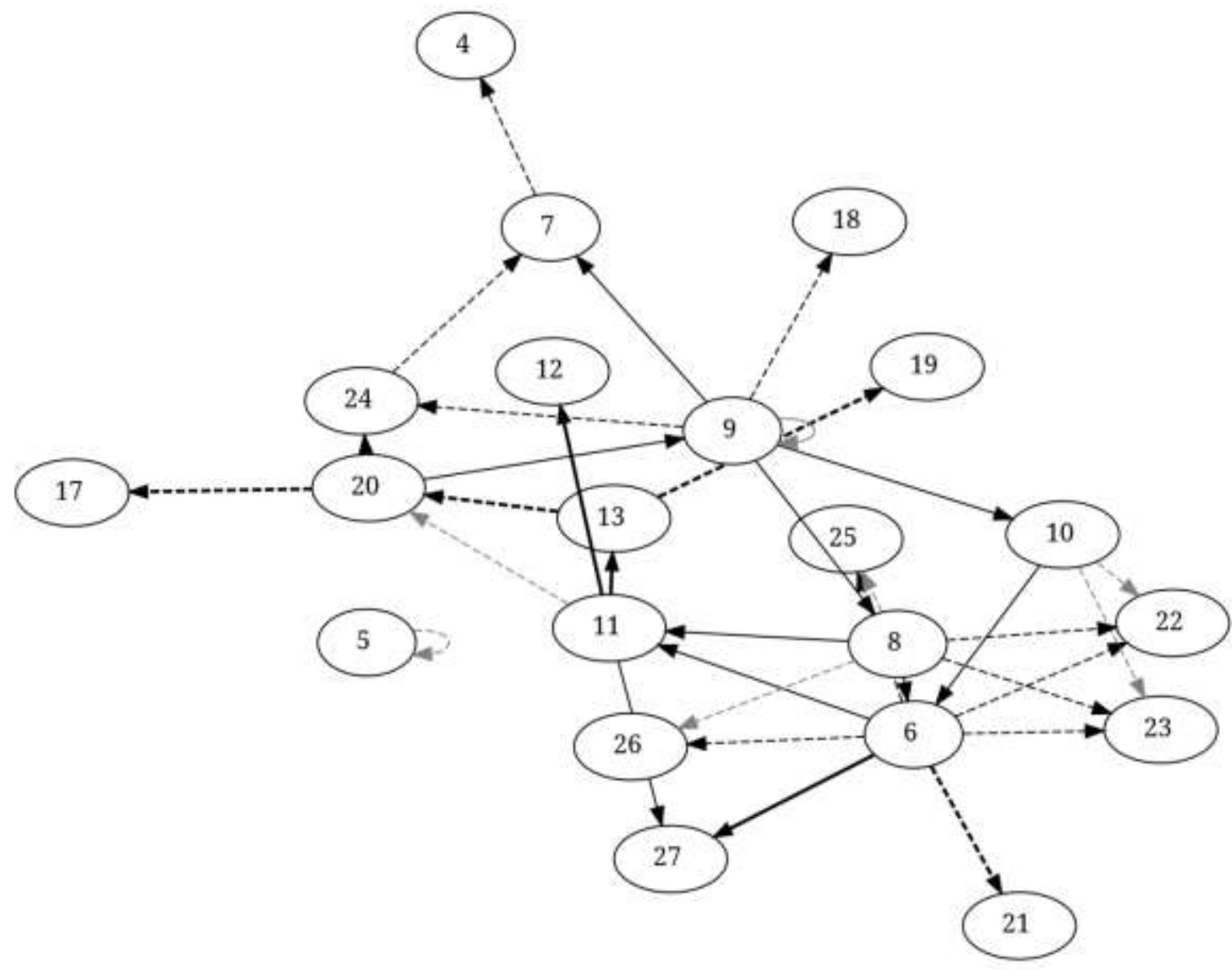

Figure 3-3 Causality network displaying anti-IgM stimulated gene expression correlations. Network modelled by B. Klaus (AG Strimmer, IMISE Leipzig), based on partial correlation using the ' $R$ ' package SIMoNe (Statistical Inference for Modular Networks (Chiquet et al., 2009)) displaying causal relationships between clusters of genes. Genes combined in one cluster display the same time course (Bayesian Hierarchical Clustering). A complete list of genes contained in each cluster can be found in Table A-1. Arrows indicate the causal relationship; bold arrows display a strong positive correlation between two clusters, whereas dashed arrows indicate a negative correlation.

In this directed graph, partial correlation between the expression courses of two clusters of genes may infer a regulatory link between the clusters. Continuous arrows indicate here a positive regulation, whereas dashed arrows represent a negative regulation. Additionally, stronger partial correlation between two nodes is depicted with bold arrows, whereas weak partial correlation is displayed with narrow arrows.

Having a closer view to the network, it becomes clear that there is no 'cluster of initiation'. All clusters depicted in Figure 3-3 are conditionally dependent on another cluster. This suggests that the anti-IgM stimulation triggered several independent processes at the same time. In contrast, several clusters are influenced by others, but do not influence a third cluster. This may be due to the limited period of time of observation and the statistical limitations in cluster generation. 
Interestingly, cluster 6 and 8 seem to influence numerous other clusters. Apparently, cluster 8 is only conditionally dependent on one Cluster (\#9) and cluster 6 is dependent on cluster 8 and 10. Both clusters (6 and 8) contain genes, which are slightly up regulated in the first 30 to 90 minutes after stimulation (Figure A-1). Both of the clusters then follow a time course, which depicts a down regulation of the harboring genes upon 90 to 240 minutes after BCR stimulation. Cluster 6 comprises 102 genes and cluster 8 harbors 58 genes. In both clusters, genes being mainly associated with signal transduction, protein modifications, transcriptional regulation and metabolic processes can be found. Cluster 6 and 8 contain amongst others genes encoding kinases (cl 6: NADK, CAMK2G, PIP4K2B, PXK, DCK, FASTK, cl 8: AURKA, P38) and genes encoding G-proteins and related proteins (cl 6: CDC20, RASGRP1, ARGHAP35, ERC1, TBC1D14, cl 8: GOPC, FPGT, ARHGEF3) (Table A-2). Both clusters 6 and 8 seem to influence the expression of genes in cluster 11, which contains 126 genes. Those genes show a similar expression course as genes from clusters 6 and 8, but with a higher expression rate, thus resulting in a distinct cluster of genes. Cluster 11 comprises genes associated with transcription, such as transcriptions factors $(M Y C, B C L 6, I D 4)$, transcriptional cofactors $(B C L 9, B C L 3)$ and others, being involved in regulation of RNA and DNA metabolic processes (Table A-2).

Apparently, statistical analysis of the here obtained time course data enable the construction of a comprehensive causality network. Unfortunately, the biological interpretation of this huge data set remains difficult, since the distinct clusters comprise large lists of genes, which cannot be assembled to one specific function (a complete overview of the GO-analyses can be found on external CD ROM IItime course analyses\Network\GO-Enrichment). Thus, the conflation of our time course specific gene expression data set with other sources of high-throughput data may be useful to fathom the biological meaning of such a causality network. In order to shed some light on the complexity of the gene expression changes and their causal correlation induced through $\alpha$-IgM stimulation, ongoing analyses are supposed to integrate genome-wide chromatin immunoprecipitation assays in our cell perturbation assays. Therewith, we aim to use such a causality network for identification of, for example, transcription factor targets. 


\subsubsection{A mathematically new method to analyze time courses identifies differentially expressed clusters of genes}

Simultaneously to the network modeling of causalities and regulations in gene expression changes over time, a second group around Prof. Dr. J. Läuter (University Magdeburg) established a new method to analyze high-dimensional time course gene expression data. The here presented strategy to provide a mathematically stringent and effective procedure to describe time course data is based on spherical tests (Läuter et al., 1996, 1998). These aim to select the variables which exhibit a statistically firm unsteady distribution over time (Läuter et al., 2012). Thereby, the following stabilizing strategy was used: genes with similar time course were pooled in one set or cluster and tested for stationarity. Those clusters of genes, which emerged to be significant (multiple Betatesting according to (Läuter et al., 1998) with alpha error $\alpha=0.05$ and abort of the testing at $k=5$ non-significant non-spherical tests (Hommel and Kropf, 2005)), can be taken for biological consideration. Hereby, the algorithm presumes that genes, which exhibit a uniform process, are potentially regulated through the same elicitor. In addition, in the case of several distinct clusters of genes, the specific shape of the serial data curves may be due to mutual regulatory dependence.

Via the adaptation and advancement of the test for stationarity, 500 sets of genes were built. Upon $\alpha$-IgM stimulation 21 sets of genes were disjoint and CD40 activation led to 20 disjoint sets of genes. 15 significant sets of genes out of 21 displaying disjoint time courses were identified for $\alpha$-lgM stimulated samples and 5 significant sets of genes for SCD40L-stimulated samples. 8 positively regulated gene groups and 7 gene groups suppressed through $\alpha$-IgM are described. CD40 activation in turn led to 4 sets of up regulated genes and one gene set showing a suppression of gene expression.

Additionally, a combined analysis of CD40 and BCR induced gene expression changes allows the comparison of congenial reactions or differences in response to the distinct stimuli. Here, gene sets were identified, in which significant differences of gene expression changes upon BCR or CD40 or in both stimulations were observable. In Figure 3-4, the blue dashed line shows the mean gene expression changes over time for CD40L-stimulated cells, whereas the red curve shows the changes upon anti-lgM antibody stimulation. Group \#1 illustrates genes highly up regulated through BCR crosslink, but not changed at all through CD40 stimulation. Additionally, group \#2 depicts genes, which showed the same course of expression changes over time, regardless which stimulus the cells experienced. Finally 10 more groups of genes could be computed showing the same directionality of gene regulation through the different stimuli, but differentiating in intensity or course after a certain time has passed. For a complete list of genes please refer to Table A-3. 
All of the computed gene sets display distinct expression changes over time, allowing discrimination between immediate-early activated gene sets, late events or immediately up regulated and later in time course down regulated gene sets.
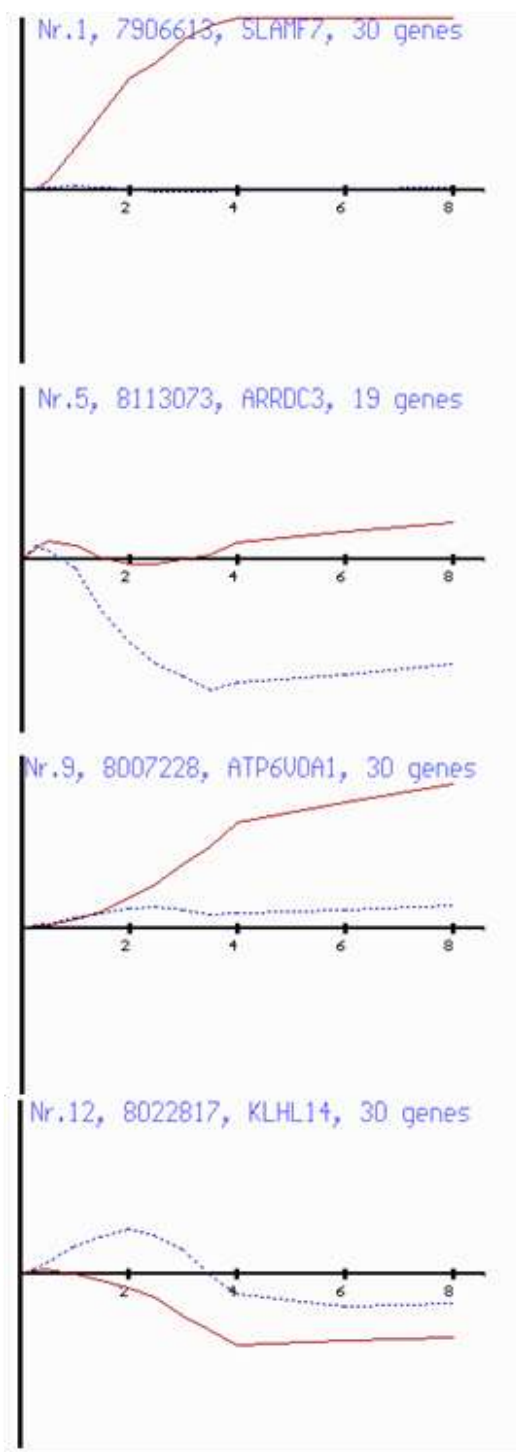
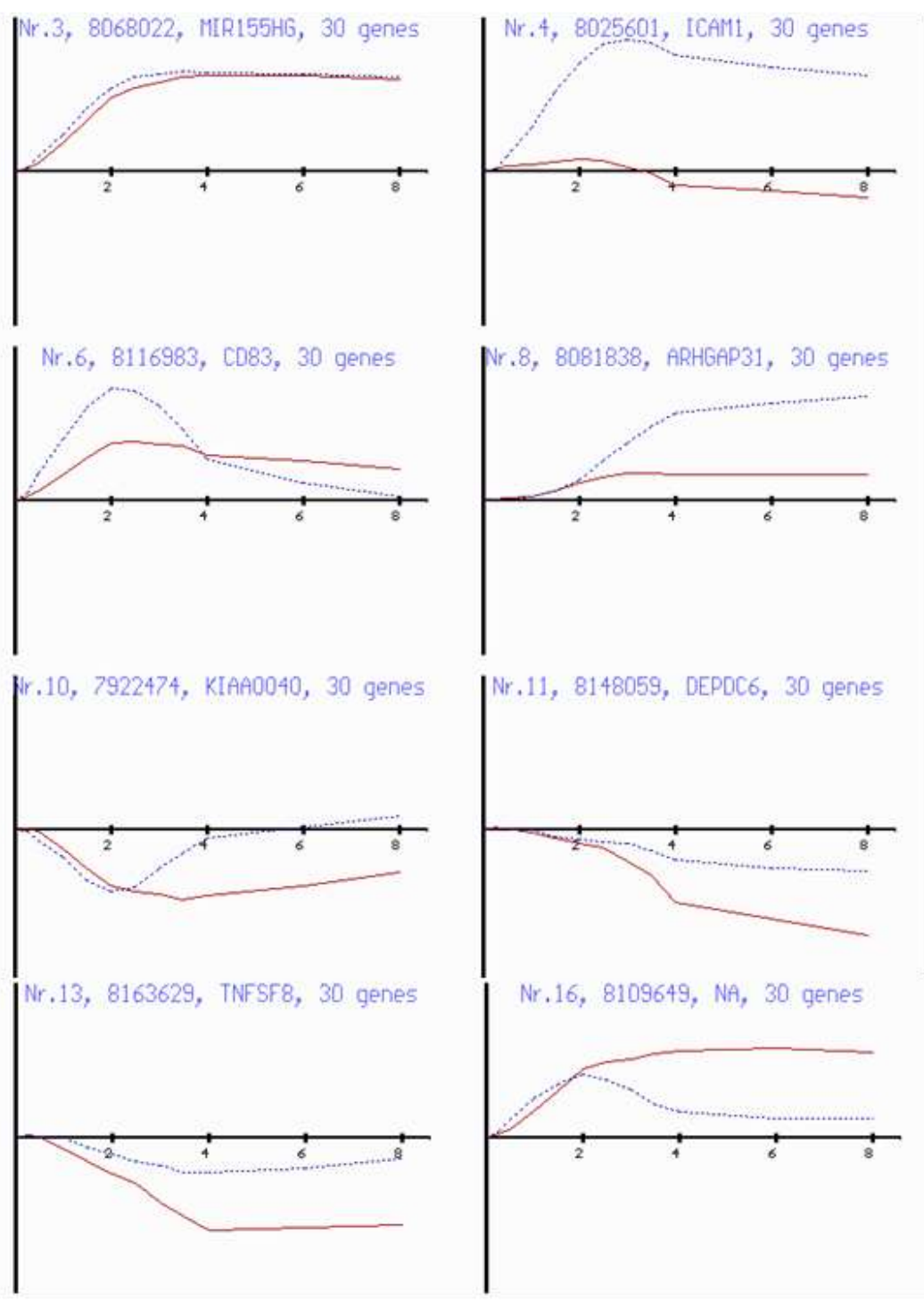
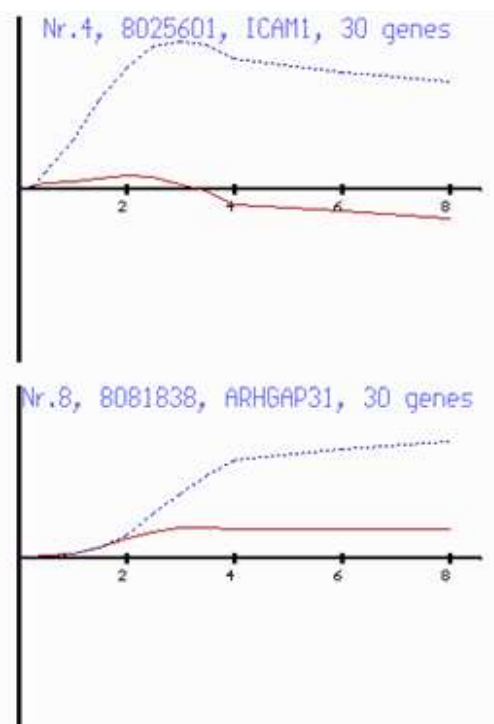

Figure 3-4 Comparison of time courses of genes commonly regulated through BCR and CD40. 12 significant, distinct groups of genes were computed, when taking commonly regulated genes by CD $40 \mathrm{~L}$ and BCR crosslink into calculation. red curve - time course of gene expression changed through anti-IgM stimulation, blue curve time course of gene expressions stimulated through SCD40L. The y-axis displays changes in gene expression levels (not scaled), x-axis shows the hours of specific treatment.

Testing for stationarity in differential gene expression changes from CD40L stimulated BL cells computed 5 disjoint and distinct gene sets (Figure 3-5 A). GO analysis was used to characterize the sets of genes. A complete overview of the GO results for each cluster of genes is provided as external data stored on a CD ROM (\\time course analyses\Stationarity\GoKeggLister). Gene set \#2 was the only group of genes, which comprised solely repressed genes. Interestingly, the majority of the gene products display tyrosine kinase or transferase activity. Taken together with the observations from 
gene set \#4, including mainly negative regulators of signaling as phosphatases, one can postulate that CD40 mediated signaling happens very rapidly on the proteome level, leading to a fast feedback loop mediated by gene expression changes. The second group of up regulated genes upon CD40L stimulation displayed a late onset of increased expression ( $>2$ hours) and may represent a second wave of target genes. In this group, GO analyses did not reveal enrichment in molecular function of different gene products, but the list contains target genes of NF-KB signaling (WNT5a, CFLAR) (Katoh and Katoh, 2009) and cytokines as TNFSF4 (OX-4OL), providing co-stimulatory signals to T-cells (Akiba et al., 1999; Brocker et al., 1999). Thus, activation of target genes affecting proliferation, differentiation and paracrine signaling occurs 2 hours after CD40L activation.

To afford the massive necessity of transcriptional regulators, a second intermediate step is imperative. Group \#8 displayed a strong increase in gene expression for the first 90 min after stimulation paired with a rapid inhibition back to basal level after 180 min after stimulation. This gene group comprised mainly DNA-binding and transcription factor activity (Table A-4 and external data on CD ROM \\time course analyses\Stationarity\GoKeggLister\CD40.3_cluster_8). In contrast, gene group \#5, showing a similar bi-phasic course with the highest expression change upon 2 to 2.5 hours after CD40L stimulation and following decline in expression levels up to basal levels upon 6 hours after stimulation, illustrated several processes as positive regulation of protein kinase activity, response to stress (unfolded proteins etc.) and expression of signaling molecules as CD40 itself (Table A-4 and external data on CD ROM \Itime course analyses\Stationarity\GoKeggLister (CD40.3_cluster_5).

Enhanced gene expression as a response to $\alpha$-IgM was found in 8 gene sets. Those groups of genes can be additionally subdivided in groups, which display an 'immediately-up' course $(\# 1, \# 2, \# 5)$ or 'late-up' gene expression (\#3, \#9, \#11, \#13,\#19). Additionally genes responding with reduced expression to $\alpha-\operatorname{IgM}$ treatment (\#4, \#6, \#12, \#17) could be computed (Table A-5). Genes in group \#1 were commonly activated upon $\alpha$-IgM stimulation and reached their highest expression level after 4 hours of stimulation (Figure 3-5 B, \#1). SLAMF7 (also known as CS1, CRACC, CD319), the center gene of the set, was strongly up regulated upon BCR crosslink, but did not respond to CD4OL stimulation (compare Figure 3-4). SLAMF7 is a lymphocyte cell surface receptor, which has been shown to be strongly up regulated in response to BCR crosslink in Ramos cells and to induce proliferation and autocrine signaling of cytokines in human B lymphocytes (Lee et al., 2007). Genes in this group could not be integrated in a specific function or process, but represent the diversity of B cell receptor signaling, immune response activating signaling and protein tyrosine kinase activity. 
A
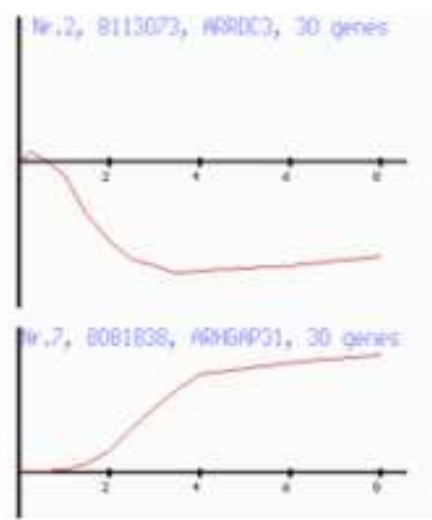

B
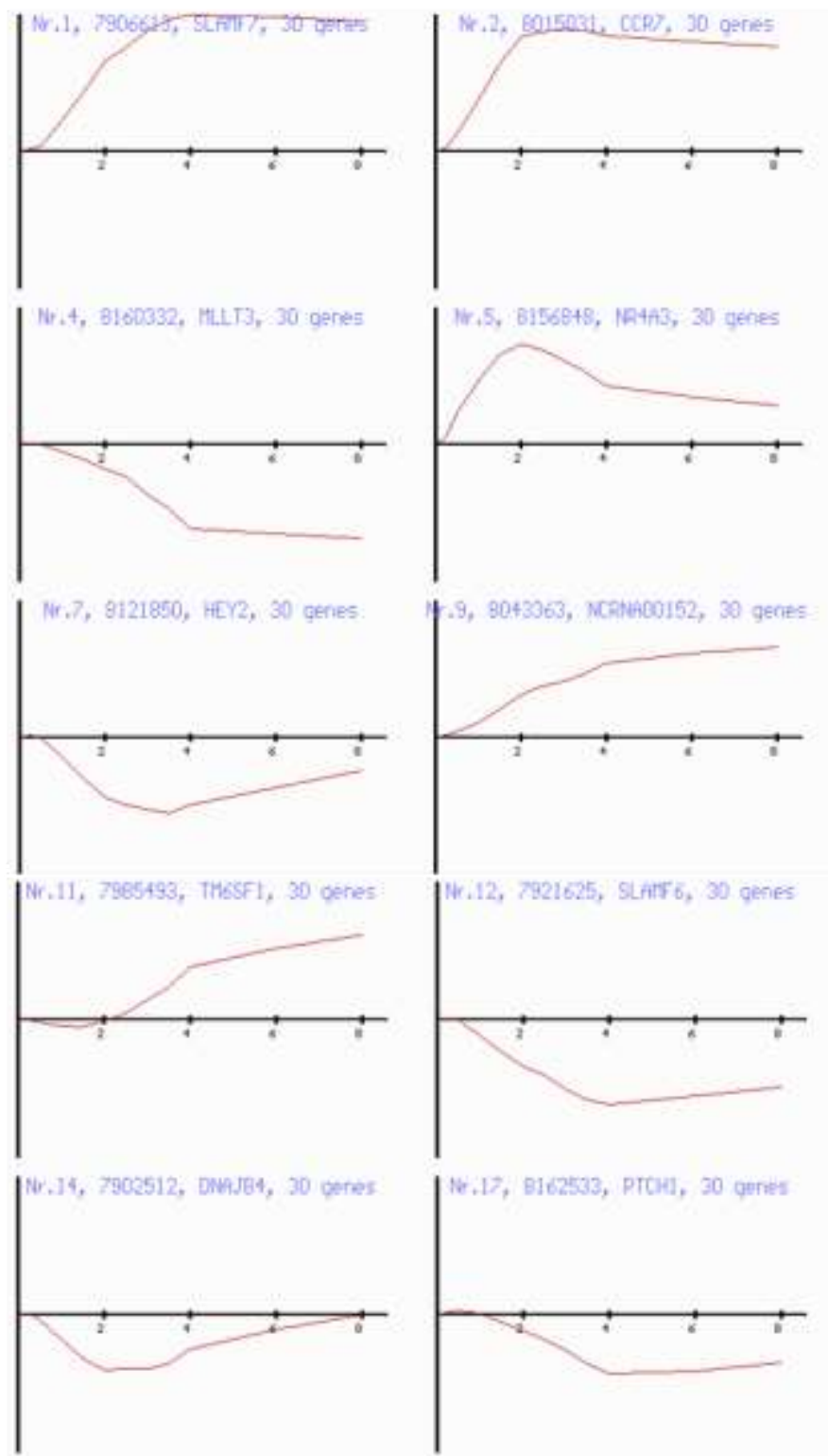
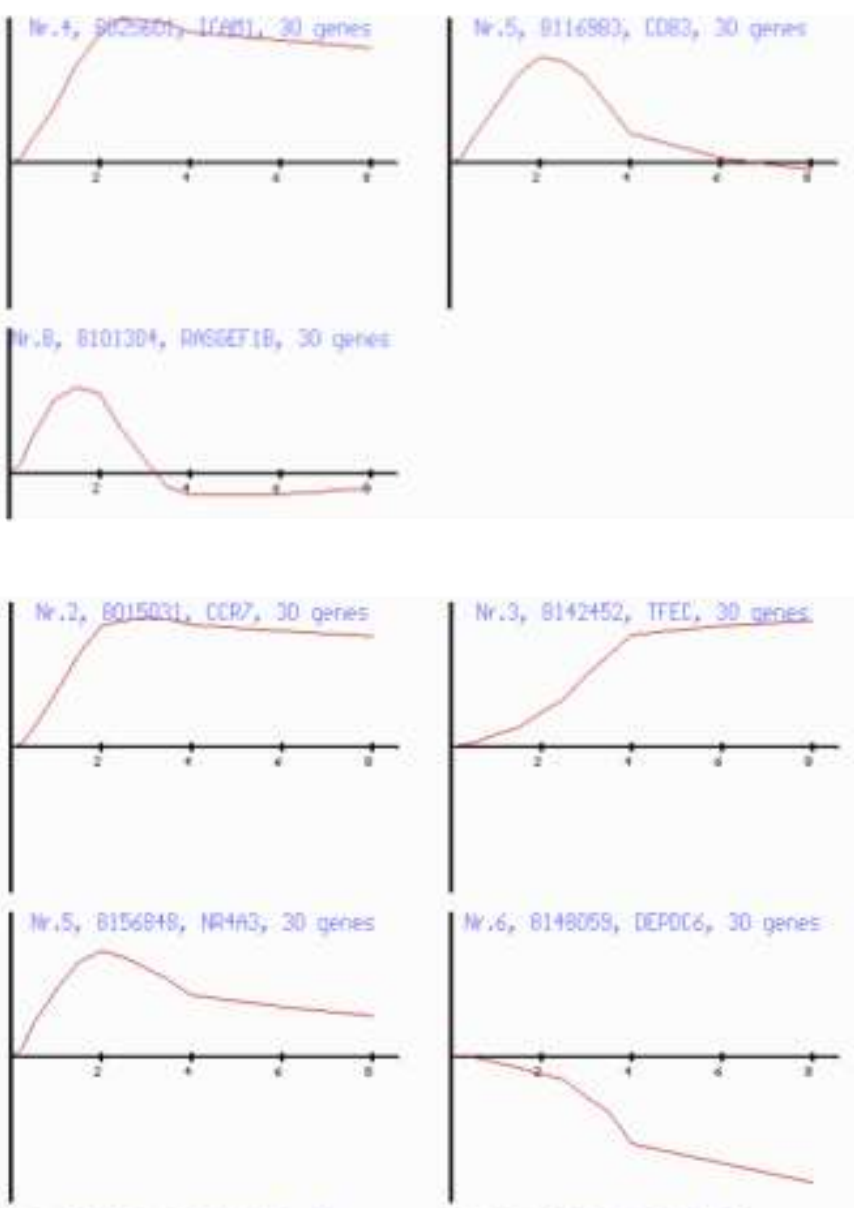

Nr.10, B029693, Fos8, 30 : genes
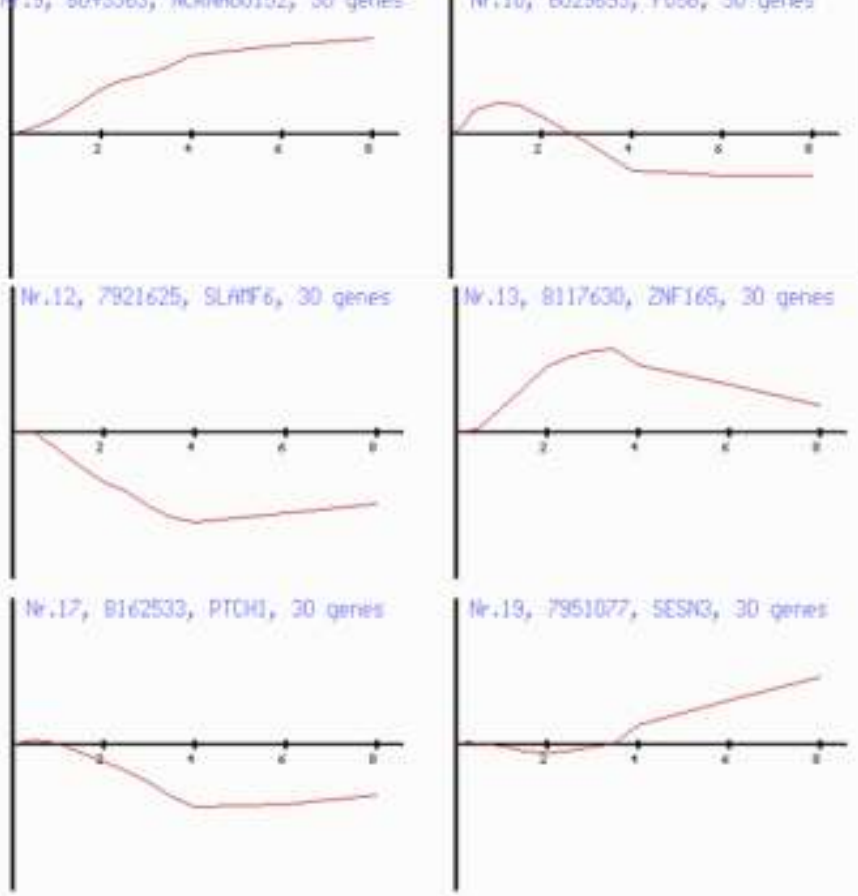

Figure 3-5 Distinct time courses for anti-IgM and CD40 stimulated BL2 cells can be determined by testing for stationarity. Testing for stationarity by reducing the number of genes in each group to 30 genes leads to $(\mathbf{A}) 5$ unique and disjoint groups of genes upon SCD40L stimulation, (B) 15 significant unique time courses upon $\alpha$ IgM stimulation of BL2 cells. The $y$-axis displays changes in gene expression levels (not scaled), $x$-axis shows the hours of specific treatment. 
The set of genes \#2 displayed a sharper increase in gene expression, reaching approx. $90 \%$ of highest expression level upon 2 hours of $\alpha$-IgM treatment (Figure 3-5 B, \#2). Center gene of the set is CCR7, a pivotal player in homing tumor cells into lymphoma-supporting niches in secondary lymphoid organs (Rehm et al., 2011) and known to be highly up regulated upon BCR stimulation (Calpe et al., 2011). Additionally this group comprised lots of genes actively sustaining immune response and playing part in MAPK signaling and G-protein-coupled signaling (\time course analyses\Stationarity GoKeggLister\BCR.3_cluster_2). Set \#5 displayed a bi-phasic course of gene expression changes. Gene expression was immediately up regulated upon $\alpha$-IgM stimulation, whereas after 2 hours expression of those genes was reduced again. This particular set of genes mainly comprised regulators of gene transcription like DNA-binding factors (PRDM2, MFSD2A, JUND, PIM3, E2F3) and genes affecting transcription factor activity (one example: $N R 4 A 3$, the center gene of the set involved in steroid hormone receptor activity and positively regulating DNA-binding transcription factor activity). This time course perfectly fits with the estimation that upon stimulation of the cell, the signal is transduced in the nucleus and gene expression is affected in a very pronounced manner, leading to feedback signals negatively regulating transcriptional activity.

A similarly interesting gene set \#10, displaying a bi-phasic time course with a slight induction of gene expression in the first hour and subsequent transcriptional repression, harbored the center gene FOSB. FOSB, belonging to the FOS gene family, encodes a leucine zipper protein, dimerizing with proteins of the JUN family, thereby forming the AP-1 transcription factor complex and regulating cell proliferation, differentiation and transformation (Entrez Gene: FOSB FBJ murine osteosarcoma viral oncogene homolog $\mathrm{B}$ ). The proto-oncogene JUNB, dimerizing partner for Fos $\mathrm{B}$, was as well regulated in gene set \#10 and displayed the same time course in gene expression. Additionally, this group harbored proto-oncogenes like PIM-1 and PIM-2, both exerting their oncogenic activity through the regulation of MYC transcriptional activity (van der Lugt et al., 1995; Shirogane et al., 1999).

Supporting this issue, the expression of $M N T$, a MAX-binding protein, seems to be co-regulated since it displayed the same time course. The protein encoded by this gene has a basic-Helix-LoopHelix-zipper domain, whereby it binds the E-box, following heterodimerization with Max proteins, thereby repressing transcription of MYC target genes (Hurlin et al., 1997a, 1997b; Sommer et al., 1998). Upon BCR stimulation, this repression is likely to be abolished, since $M N T$ expression levels were decreasing (Figure 3-5 B, \#10). The gene groups \#3, \#9 and \#13 comprise genes whose expression was up regulated after $\alpha$-IgM stimulation later in time. In general, the increase in gene expression took a longer period of time than in the groups mentioned above. Gene set \#9 displayed a continuous gain of gene expression, reaching a saturation level after 4 hours of stimulation with $\alpha$ IgM antibody. Genes encountered in this group could not be assigned to one single specified 
biological process, but their products were mainly membrane- and organelle-bound proteins. One example is LRMP (lymphoid-restricted membrane protein, Jaw1), an endoplasmic reticulumassociated protein, which was originally identified as differentially regulated transcript in B and T cells (Behrens et al., 1994). Subsequently, LRMP was reported as marker for diffuse large $\mathrm{B}$ cell lymphoma of the GCB subtype (Tedoldi et al., 2006).

In gene set \#3, gene expression was constitutively increasing for 2 hours after BCR crosslink reaching a point of inflection after 4 hours of stimulation. Genes comprised in this particular gene set comprised mainly regulating kinase or transferase activity (\lime course analyses\Stationarity\GoKeggLister\BCR.3_cluster_3), thus maybe inducing a "second wave of activity". Such kinases might act as feedback loop for the immediate-early response genes. The expression of genes in group \#13 increased slowly upon BCR crosslink, reaching the maximum after 4 hours of stimulation before being reduced again in direction to basal level of expression. The high diversity of genes comprised in this group impeded the assignment of one specific function to this cluster. Among them, we found genes whose products represent several functions such as Rho protein signal transduction (ARAP2), DNA binding components (ZNF165, PHF13, ZNF643), helpers in protein unfolding (TOR3A) and others ( $\backslash$ Itime course analyses\Stationarity\GoKeggLister (BCR.3_cluster_13). Finally, group \#11 and \#19 show the genes, whose expression was increased at very late stages in the observation period. In group \#11, genes preparing the cellular machinery for translational processes were enriched (AGA, TARS, WARS, UGDH), whereas in group \#19 the negative regulation of the cellular response to growth stimuli was activated (NEK7, CLCC1). Thereby, negative feedback loops of early-activated genes are potentially activated.

Additionally, 6 groups of genes showed a decrease in gene expression upon anti-lgM stimulation. The groups \#4 and \#6 showed a similar course where expression continuously diminished as time went on. In contrast, in groups \#7, \#12, \#14 and \#17, a bi-phasic expression course could be observed. In the first phase, gene expression decreased to be returned after a certain period of time to the initial expression level. Since the basal expression level was not reached during the period of observation and the gain in expression took place very slowly, one might postulate that this time course depicts the time-resolved turnover of mRNA abundance in the cell: after initial regulation (here repression), the inhibitory effect is released and slowly mRNA expression is increased again, leading back to the point of departure.

In gene set \#7, center gene of the set was HEY2, which encodes a bHLH transcription factor. The encoded protein acts as a transcriptional repressor and binds preferentially to the canoncical $\mathrm{E}$ box sequence 5'-CACGTG-3' (Heisig et al., 2012; Wu et al., 2011). Interestingly, the expression of this gene was down regulated following anti-lgM stimulation, thus promoting a preexisting release of 
transcriptional repression. $\mathrm{GO}$ analysis also showed enrichment for transcriptional regulation and negative regulation of cell growth (\time course analyses\Stationarity\GoKeggLister (BCR.3_cluster_7) in gene set \#7. A similar enrichment could be found in gene sets \#12, \#14 and \#17. Thus, negative regulators of gene transcription were down regulated through BCR crosslink, promoting active gene transcription. Interestingly, gene set \#17 harbored the genes PTCH1, LEF1 and NOTCH1. All three genes are known to be evolutionary important for developmental processes as hematopoiesis. Notch1, the transmembrane receptor for the Notch signaling network, plays a pivotal role in lymphocyte development, proliferation and differentiation (Anderson and Longnecker, 2009; Cheng et al., 2001; Pui et al., 1999; Radtke et al., 2004). Lef1, a transcription factor participating in the Wnt signaling pathway, regulates B lymphocyte proliferation (Reya et al., 2000) and is aberrantly expressed in BLs (Hummel et al., 2006; Walther et al., 2013). Ptch1 is a transmembrane receptor for hedgehog signaling and plays an important role in lymphoid lineage commitment (Uhmann et al., 2007) and the germinal center reaction of B cells (Sacedón et al., 2005). Furthermore, recent publications show deregulations of the Hh signaling pathway in several lymphoma subtypes, including DLBCLs (Decker et al., 2012; Lin et al., 2010; Siggins et al., 2009; Singh et al., 2009, 2010). The $\alpha$-IgM stimulation of BL cells led to reduction of expression of NOTCH1, LEF1 and PTCH1, suggesting an active signaling in BL. Similarly, gene expression of $c-M Y C$, the hallmark of BL was down regulated after BCR crosslink (gene set \#14).

Taken together with the observation, that $\alpha$-IgM stimulation of $B L$ cells reduced the $\mathrm{mBL}$ index towards a more DLBCL-like index (compare chapter 3.1.1), the question arose whether the down regulation of those signaling pathways may give an explanation for essential differences between BL and DLBCLs. For a better understanding of the joint $\alpha$-IgM mediated suppression of PTCH1, LEF1, NOTCH1 and as well of MYC, I focused in this study on the role of PTCH1 in aNHL. Recent publications suggest an important, but yet not clearly understood role for Hedgehog signaling in lymphoma genesis and progression (Decker et al., 2012; Singh et al., 2009, 2010). The role of LEF1 and MYC in the diversion of $B L$ and $D L B C L$ is part of other projects in our group. 


\subsection{Ptch1 and Hedgehog signaling in lymphoma}

Using qRT-PCR it was verified that the statistically modeled data are correct and that MYC as well as PTCH1 and LEF1 expression is down regulated after BCR crosslink. Therefore, RNA used for array hybridization (batches I-III, Figure 3-6) as well as independently collected data (data not shown) showed a marked down regulation of PTCH1, LEF1 and MYC upon BCR activation. Although not postulated through the course of gene set \#17, PTCH1 expression showed a two-fold up regulation in the first hours after stimulation. This discrepancy between computed time course and time course collected through qRT-PCR may be due to the calculation of the mean time course of all genes in gene set \#17. Additionally, the 3 batches of stimulated BL cells used for array hybridization differed strongly in their response to BCR activation regarding PTCH1 mRNA expression especially in the first two hours of data collection. For statistical modeling an adaptive 3-point smoothing was applied, thus probably removing those differences in favor of the not-elevated mRNA expression data. However, an overall agreement of the computed to the experimental data could have been shown. PTCH1, MYC and LEF1 mRNA expression was reduced in BL2 cells after 3 hours after $\alpha$-lgM antibody treatment. This reduction lasted until 5-6 hours after stimulation and was then abolished (Figure 3-6).

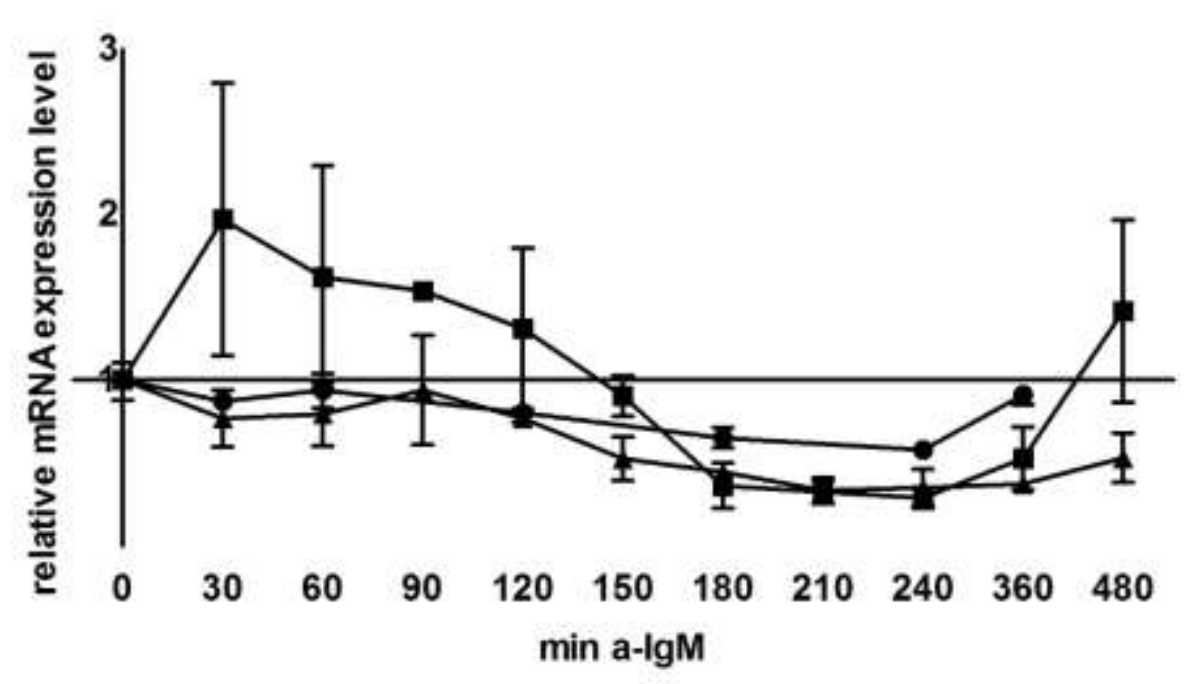

Figure 3-6 MYC, PTCH1 and LEF1 mRNA expression is suppressed in BL2 cells upon anti-IgM stimulation. Relative mRNA expression levels of $c-M Y C, P T C H 1$ and $L E F 1$ as a function of time upon stimulation with $\alpha$-lgM $\mathrm{F}(\mathrm{ab})_{2}$ fragment. Data are represented as mean ( \pm SEM) of three biological replicates (batches I-III, which were subjected to microarray analyses). $2^{-\Delta \Delta C t}$ values were calculated using $A B L$ as housekeeping gene. 


\subsubsection{Expression profile of Hedgehog signaling components}

Recent findings suggest that hedgehog signals exerted from the microenvironment are important in hematologic malignancies, including lymphomas (Dierks et al., 2007; Hegde et al., 2008). All Hh ligands (Sonic hedgehog (Shh), Indian hedgehog (Ihh) and Desert hedgehog (Dhh)) share a common signaling pathway, where Patched (Ptch1) and Smoothened (Smo) are the membrane bound receptors. In the absence of ligand, Ptch1 exerts an inhibitory effect on Smoothened activity that is abrogated upon Hh ligand binding. Subsequent activation of the Gli transcription factors, Gli1, Gli2 and Gli3, leads to expression of PTCH1 and GLI1 amongst others. Recently, Singh et al. (2010) have shown that several DLBCL cell lines express PTCH1, GLI1 and SMO and that inhibition of Hh signaling via inhibition of Smo through cyclopamine resulted in cell-cycle arrest and apoptosis in some of the tested DLBCL cell lines (Singh et al., 2010). To investigate whether Hh signaling components are expressed in a similar manner in Burkitt's and diffuse large B cell lymphoma cell lines, the mRNA expression profile of PTCH1, GLI, SMO, SHH and IHH was comprehensively analyzed using qRT-PCR. Additionally, the reduction in mRNA expression levels of PTCH1 upon 3 hours $\alpha$-IgM F(ab) fragment $_{2}$ in BL2 cells was counterchecked in several cell lines of Burkitt's and diffuse large B cell lymphoma (Figure 3-7). mRNA expression levels are depicted as fold changes relative to the unstimulated cell line with the lowest expression level of the respective mRNA (marked with an asterisk in Figure 3-7).

As shown in Figure 3-7 A, the observed reduction of PTCH1 mRNA upon $\alpha$-IgM treatment for 3 hours in BL2 cells was not reproducible in other Burkitt's lymphoma cell lines tested, except BL30. All Burkitt's lymphoma cell lines tested did express PTCH1 mRNA (Ct-values around 25 cycles, housekeeper ABL 21 cycles), but most of them showed slightly elevated PTCH1 mRNA expression levels upon BCR crosslink. Diffuse large B cell lymphoma cell lines showed approximately the same overall expression levels as $B L$ cell lines, however, the response to $\alpha$-IgM treatment for 3 hours in terms of PTCH1 mRNA expression levels differed. SUDHL5, SUDHL6 and OCI-Ly3 showed a slight to moderate decrease in PTCH1 mRNA expression upon BCR activation, whereas the other DLBCL cell lines tested did not change or slightly increased their PTCH1 mRNA expression levels (Figure 3-7E). PTCH1 gene expression is amongst others activated through the successful activation of the $\mathrm{Hh}$ signaling pathway as a target gene of Gli1 transcription factor (Marigo and Tabin, 1996). The transcription factor GLI1 itself is also a well-known target gene of Hh signaling (Lee et al., 1997). Therefore, the mRNA expression levels of $h G L I 1$ were investigated in BL and DLBCL cell lines before and 3 hours after BCR crosslink. 


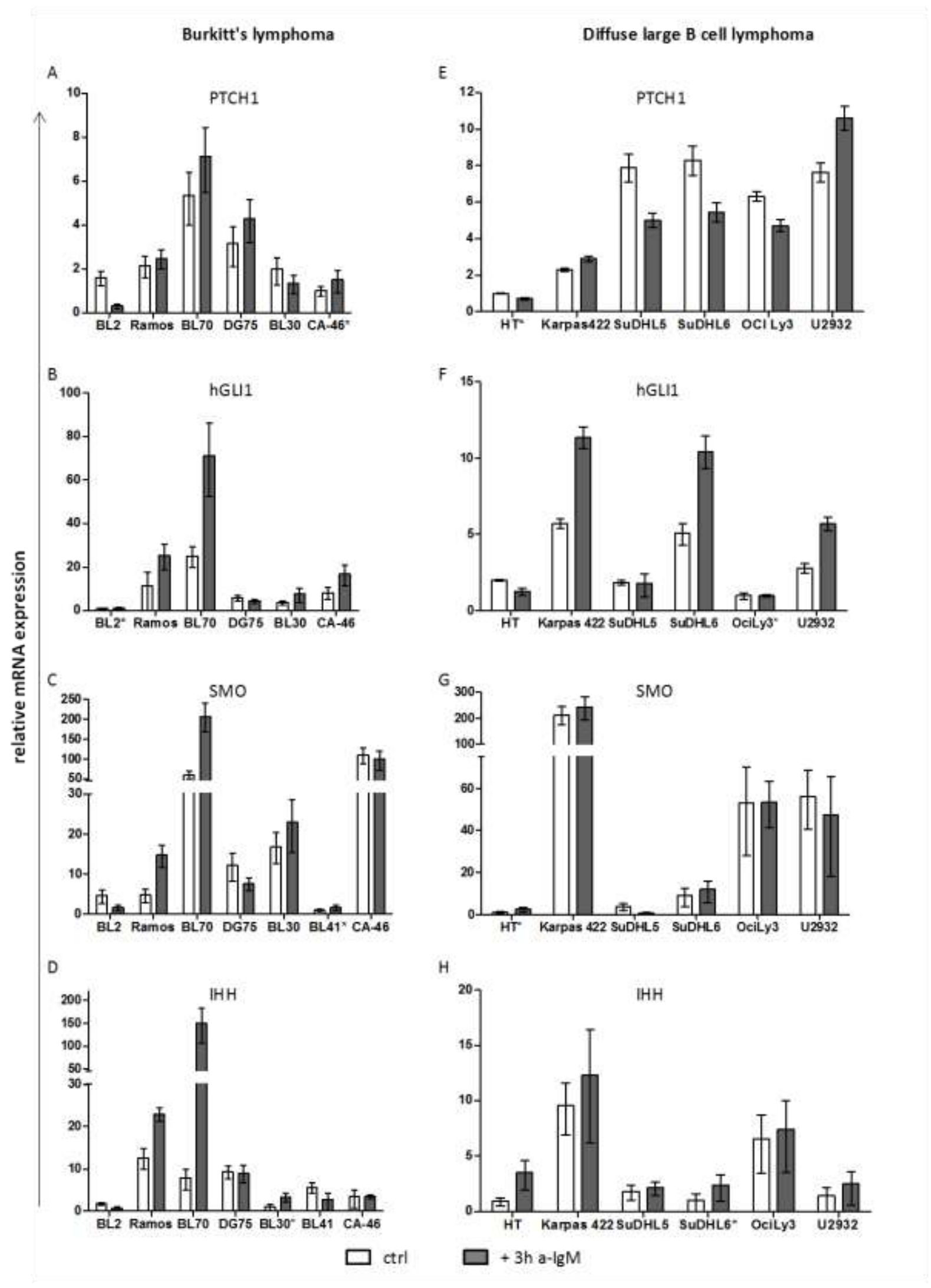

Figure 3-7 Differential expression and induction of Hh signaling components in Burkitt's and diffuse large B cell lymphoma cell lines through anti-lgM stimulation. Several Burkitt's lymphoma (A-D) and diffuse large B cell lymphoma (E-H) cell lines were tested for expression of hedgehog signaling pathway components by qRTPCR. Additionally, cell lines were stimulated 3 hours with anti-lgM $F(a b)_{2}$ fragment and gene expression was compared to unstimulated control. Results are represented as $2^{-\Delta \Delta C t}$-value relative to $A B L$ housekeeper 
expression and normalized to the cell line with the lowest expression (marked with an *). One representative experiment out of 3 biological replicates is shown. Expression of SHH mRNA could not be detected in any of the tested cell lines.

Overall mRNA expression levels were pretty low in most BL cell lines (Ct-values around 30 cycles, housekeeper $\mathrm{Ct}=21)$ and differed largely between the cell lines $(25$-fold more basal hGLI1 mRNA expression in BL70 than in BL2, Figure 3-7B). One could observe an increase of mRNA expression after BCR crosslink in nearly all BL cell lines (except DG-75), which is not correlated to the response to BCR in terms of PTCH1 expression. Both genes are meant to be target genes of activated $\mathrm{Hh}$ signaling, but in BL2 cells PTCH1 expression was reduced upon anti-IgM stimulation whereas hGLI1 expression increased. In half of the BL cell lines tested (Ramos, BL70 and CA-46), one could observe a concomitant increase in mRNA expression, which would suggest an activation of Hh signaling pathway through BCR activation. In contrast, in 3 out of 6 BL cell lines (BL2, DG75, BL30) the regulation of these two target genes PTCH1 and hGLI1 was opposed (Figure 3-7 B). Similar observations have been made for the target gene expression of activated Hh signaling in DLBCL cell lines. The basal expression of $h G L I 1$ mRNA was in all DLBCL cell lines tested pretty low (Ct-values around 30 cycles), with differences of 5-fold higher expression in Karpass422 and SUDHL6 than in the DLBCL cell line with the lowest expression, OCI Ly3 (Figure 3-7 F). Here, the cell lines with the highest basal expression level (3/6) also showed an increase in hGLI1 mRNA expression after 3 hours $\alpha$-IgM stimulation, whereas the others did not respond in terms of GLI1 mRNA expression level changes (OCI Ly3 and SuDHL5) or did show reduced levels (HT). Overall, one could not observe a consistent regulation of Hh signaling target genes upon BCR activation in the tested cell lines.

Furthermore, the intracellular signal transducer SMO was expressed in strongly diverse levels when comparing different BL cell lines (Figure 3-7 C). In some BL cell lines, expression of SMO was barely detectable (BL41, Ramos, Ct-values around 33 - 35 cycles), whereas in other BL cell lines a 60to 100 -fold higher basal expression level was observed (BL70, CA-46). The response to BCR induced signaling in terms of $S M O$ regulation, regarding the different $B L$ cell lines was comparatively differential. 2/7 cell lines (BL2 \& DG75) showed an reduced expression level upon 3 hours $\alpha$-IgM stimulation, 2/7 BL cell lines (BL41 \& CA-46) did not show a regulation of SMO and the remaining 3 cell lines (Ramos, BL70 and BL30) increased the SMO expression levels upon BCR crosslink. In contrast, DLBCL cell lines, which also showed strong differences in basal SMO mRNA expression, showed no response to BCR induced signaling in terms of SMO expression (Figure 3-7 G).

When investigating the expression profile of Hh ligands, I focused on the expression of $\mathrm{SHH}$ and $I H H$, because of their implication in lymphocyte biology (Dierks et al., 2007; Lindemann, 2008; Sacedón et al., 2005; Siggins et al., 2009). Interestingly, no SHH mRNA expression was detectable at 
all via use of SYBR based qRT-PCR and the expression levels of $I H H$ were very low in BL and DLBCL cell lines tested. Investigating the expression changes of $I H H$ mRNA after 3 hours $\alpha$-IgM stimulation, differences ranging from more than 15 -fold increase in IHH mRNA (BL70) to no change (DG75, CA-46) or reduction in $I H H$ mRNA expression (BL2) were observable in $\mathrm{BL}$ cell lines (Figure 3-7 D). Overall, Burkitt's and diffuse large B cell lymphoma cell lines demonstrated very inconsistent basal levels as well as a very heterogeneous response to $\alpha$-IgM stimulation regarding the expression profile of $\mathrm{Hh}$ signaling components. A clear activation or inactivation of the Hh signaling pathway through $\mathrm{BCR}$ induced signaling was not detectable.

Although literature concerning the expression and secretion of Hh ligands in hematologic malignancies is somewhat controversy, Dierks et al. showed that hedgehog ligands produced by stromal cells support proliferation and survival of B cell lymphomas (Dierks et al., 2007). Additionally, Singh et al. reported that DLBCL cell lines (OCI Ly3) expresses and secretes $\mathrm{Hh}$ ligands (Singh et al., 2010). Therefore, we decided to test $B L$ and $D L B C L$ cell lines for responsiveness to Shh and activatability of the Hh signaling pathway.

\subsubsection{Lymphoma cell lines neither secrete nor respond to Sonic hedgehog}

The murine B cell hybridoma cell line B9 (kind gift of Prof. Dr. H. Hahn), being highly responsive to Shh as Hh ligand, was used to investigate the capability of several lymphoma cell lines to produce Shh. A HEK293 cell line constitutively secreting Shh (HEK293SHH, a kind gift of Prof. Dr. H. Hahn) was used to produce conditioned media, enriched with soluble Shh. Subsequently, after having cultivated the murine B9 cells with conditioned media for 24 hours, the mRNA expression levels of mGLI1 were measured using qRT-PCR as a mean of activated Hh signaling. As shown in Figure 3-8 A, expression of mGLI1 markedly increased upon cultivation of B9 cells with Shh-containing conditioned media obtained from HEK293SHH. Simultaneously, conditioned media from BL2, Ramos and OCI Ly3 cells were tested for a comparable potency in activating Hh signaling in murine B9 cells, which would suggest a Shh secretion of the lymphoma cells. As clearly depicted in Figure 3-8 A, none of the tested B cell lymphoma cell lines was capable of activating mGLI1 expression in B9 cells, thus indicating no Shh secretion into the medium.

To investigate the hypothesis that B cell lymphoma cells are sensitive to stroma-initiated $\mathrm{Hh}$ signaling, B cell lymphoma cell lines were incubated with Shh-containing media for 24 hours and the expression level of hGLI1 was monitored as target gene of activated Hh signaling. As depicted in Figure 3-8 B, none of the tested cell lines changed the $h G L I 1$ expression levels upon Shh stimulation. Therefore, one can conclude that those B cell lymphoma cell lines are not sensitive to secreted Shh as potent activator of Hh signaling. 
Several inhibitors of $\mathrm{Hh}$ signaling have been identified and are an extensive field of research for pharmaceutical companies and universities. The majority of the Hh pathway inhibitors reported today target Smoothened (Mar et al., 2011). One of the first inhibitors discovered is the Smo antagonist cyclopamine, which abrogates $\mathrm{Hh}$ signal transduction and therefore inhibits target gene expression (Incardona et al., 1998). To investigate the ability of cyclopamine to change gene expression levels of PTCH1 and hGLI1 upon stimulation with $\alpha$-IgM antibody, Burkitt's lymphoma cell lines were treated for one hour with cyclopamine and subsequently stimulated for 3 hours with $\alpha$ IgM antibody. PTCH1 and hGLI1 mRNA expression levels were subsequently analyzed by qRT-PCR (Figure 3-8 C \& D).
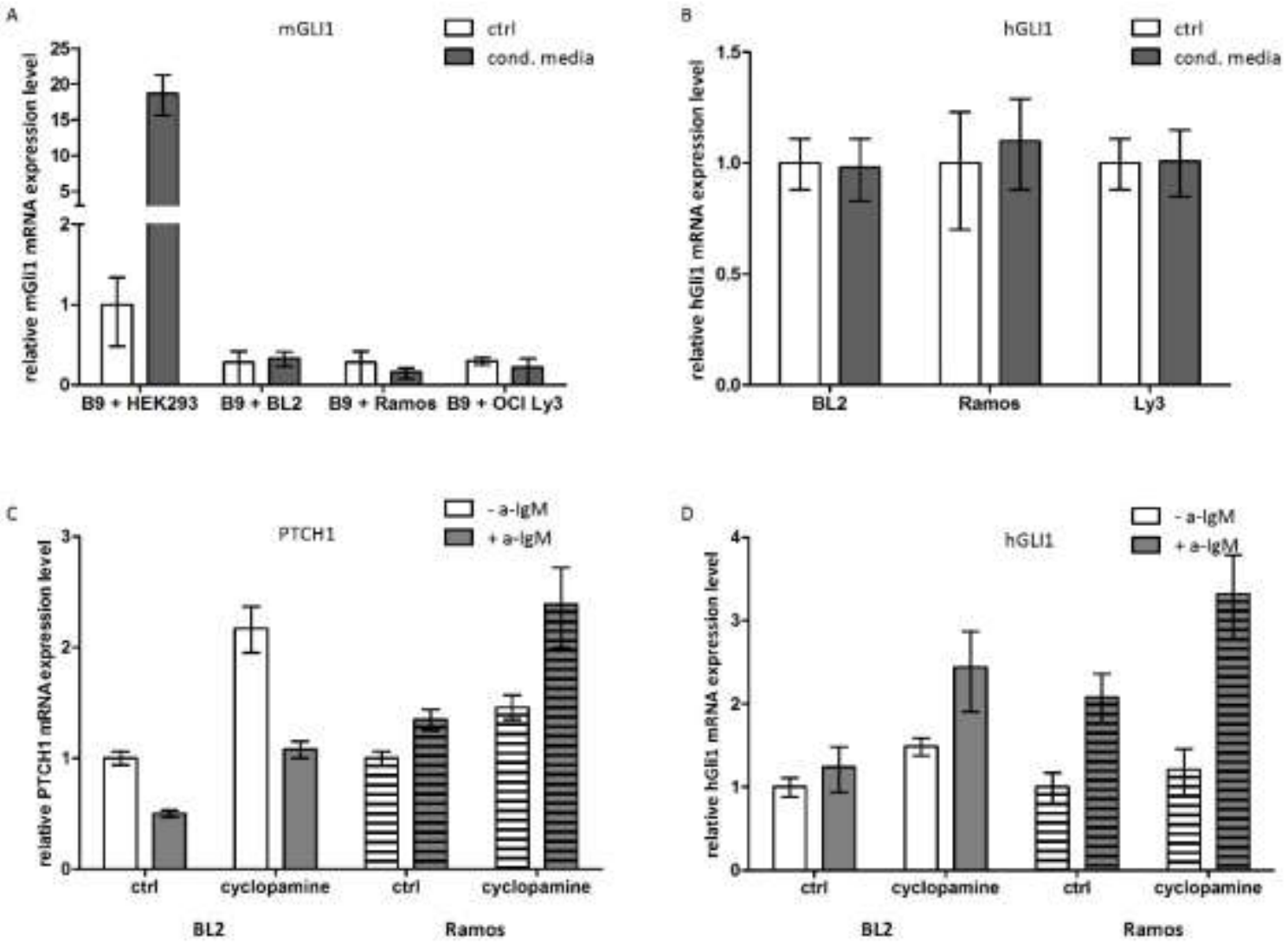

Figure 3-8 Lymphoma cell lines do not produce Shh, neither do they respond to Shh nor to cyclopamine. A Secretion of Shh through lymphoma cell lines was tested using the model cell line B9, being highly responsive to Shh. Murine B9 cells were 24 hours incubated with conditioned supernatant of Shh-secreting HEK293 cells as positive reference for $m G L / 1$ expression. Conditioned media of Burkitt's and diffuse large B cell lymphoma cell lines was also tested to induce $m G L 11$ expression by qRT analysis. B Lymphoma cell lines are not responsive to secreted Shh in regard to Hh signaling pathway activation. BL2, Ramos and OCI Ly3 cells were incubated with conditioned media of Shh-producing HEK293 cells and their expression of hGLI1 was determined by qRT analysis. C + D Neither BL2 nor Ramos cells are responsive to Cyclopamine. Cells were treated with $5 \mu \mathrm{M}$ cyclopamine for 1 hour, and additionally stimulated with $\alpha$-IgM $\mathrm{F}(\mathrm{ab})_{2}$ fragment for 3 hours. Relative mRNA expression of PTCH1 (C) and $h G L I$ (D) was measured using quantitative real-time PCR. Data were analysed for all samples using the $\Delta \Delta \mathrm{Ct}$-method. Shown are representative results of at least two experiments, relative to $A B L$ housekeeper expression. 
As shown in Figure 3-8 C, the response of BL2 and Ramos cells to cyclopamine treatment differed with regard to PTCH1 expression. In BL2 cells, basal PTCH1 mRNA expression levels were elevated upon cyclopamine treatment (2-fold increase), but the BCR induced repression of PTCH1 mRNA expression was not affected through cyclopamine treatment. In Ramos cells contrasting results were obtained. The BCR signal led to slight induction of PTCH1 mRNA expression, but the cyclopamine treatment did not change the basal PTCH1 expression. Instead an increase of PTCH1 mRNA expression upon cyclopamine treatment and subsequent BCR crosslink was observable. This observation emphasizes once again the heterogeneity of $\mathrm{Hh}$ signaling responsiveness between different cell lines of one lymphoma subtype and leads to the overall conclusion that active canonical Hh signaling may not play an important role in Burkitt's lymphoma.

\subsubsection{PI3K-mediated regulation of PTCH1, c-MYC and LEF1 mRNA expression}

Initially, the observation that the $\alpha$-IgM stimulation in BL2 cells led to a down regulation of PTCH1 mRNA expression, raised the hypothesis that Burkitt's lymphoma harbor active Hh signaling. The transition of the $\mathrm{mBL}$ index from $\mathrm{mBL}$ towards DLBCL through $\mathrm{BCR}$ crosslink would then suggest an inactivation of $\mathrm{Hh}$ signaling in $\mathrm{DLBCL}$. However, several publications have reported quite the opposite: active Hh signaling promoting lymphoma growth and progression, especially in DLBCLs (reviewed in Ok et al., 2012). Indeed, the results presented in the current study, showing enhanced GLI1 expression upon BCR activation would favor the hypothesis of active Hh signaling in the transition to DLBCL instead of an inactivation. Nevertheless, the observation of reduced PTCH1 mRNA levels was accompanied by similarly reduced levels in LEF1 and c-MYC mRNA expression. We therefore asked whether the regulation of these three genes may be directly targeted by $\mathrm{BCR}$ crosslink. Indeed, several very recent reports, being published in the course of the present study, supported our idea. Schmitz and coworkers reported about tonic BCR signaling and PI3K activity in Burkitt's Lymphoma (Schmitz et al., 2009) and Sander and colleagues established a new murine model supporting the view of synergistic action between c-Myc and PI3K signaling (Sander et al., 2012).

As shown in Figure 3-9 A, PTCH1 mRNA expression was reduced upon $\alpha$-IgM stimulation, whereas inhibition of the BCR signal transduction through the PI3K-inhibitors Ly294002, Compound15e (C15e) and BKM120 did not change PTCH1 mRNA levels in comparison to unstimulated cells. Additionally, the PLCY-inhibitor U73122 as well as the PKC-inhibitor Bisindolylmaleimide I abrogated the BCR induced decrease in PTCH1 mRNA expression. Similar observations were made for the regulation of $c-M Y C$, although here, an inhibition of the PKC seems to affect the basal c-MYC mRNA expression level positively (2.5-fold up regulation of $c-M Y C$ upon Bisindolymaleimide inhibitor use) (Figure 3-9 B). In former experiments, cells were treated 3 hours with inhibitor before subsequent stimulation. 
This led to elevated PTCH1 and c-MYC mRNA levels upon PI3K-inhibitor use (Ly294002, C15e and BKM120, respectively) in unstimulated BL2 cells (Schrader et al., 2012 and unpublished own data). To reduce these effects, the inhibitor treatment was reduced to 30 min prior to 3 hours stimulation together with respective inhibitor treatment.

A

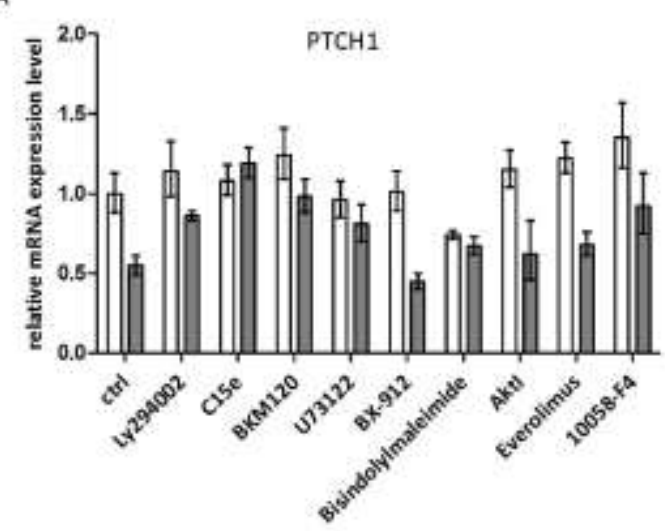

c

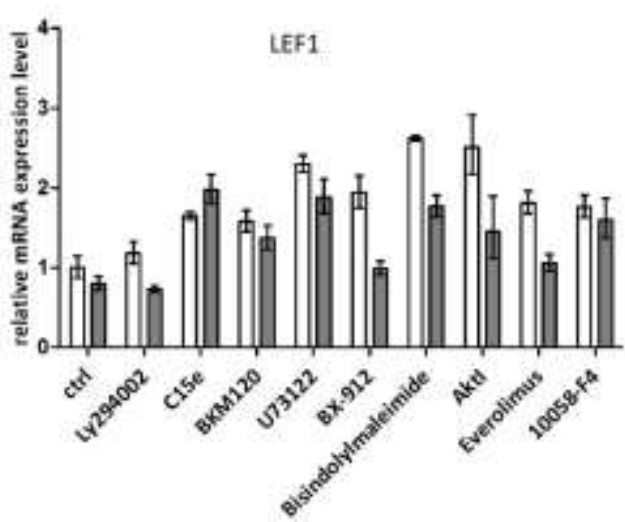

B

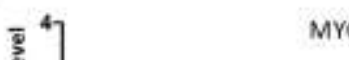

MYC

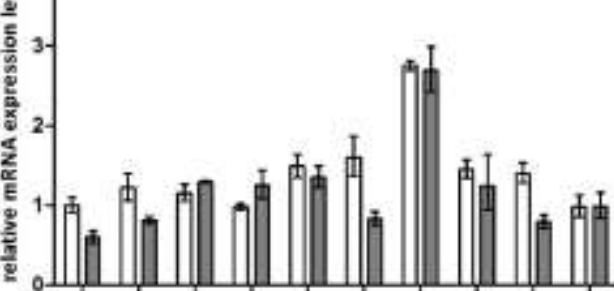

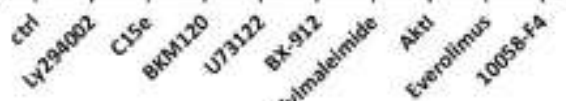

$\square$-a-lgM $\square+a-\lg M$

Figure 3-9 Regulation of PTCH1, MYC and LEF1 is mediated through PI3K-PLC $\boldsymbol{P}$-PKC signaling. BL2 cells were subjected to $30 \mathrm{~min}$ treatment with inhibitors and subsequently 3 hours of stimulation with $\alpha-\operatorname{lgM} F(a b)_{2}$ fragment. mRNA expression levels of PTCH1 (A), c-MYC (B) and LEF1 (C) were determined using the $\triangle \triangle C \mathrm{Ct}-$ method, relative to $A B L$ housekeeping mRNA expression levels. Data are represented as mean of three biological replicates ( \pm SEM), except for 10058-F4, which was used only twice.

Additionally, the reduction in MYC mRNA levels after BCR activation is abolished upon Akt inhibition. Thus the c-MYC regulation might be situated downstream of Akt-signaling, whereas PTCH1-regulation seems to be Akt-independent. Interestingly, none of both is regulated through the mTOR-pathway, interrupted by Everolimus. The inhibitor of Myc-Max dimerization 10058-F4 was used to investigate the effect of c-MYC regulation onto PTCH1 and LEF1 gene expression. $\alpha$-IgM mediated MYC suppression is reversed by the Myc-inhibitor 10058-F4, whereas PTCH1 repression seems to be only slightly affected. For LEF mRNA expression it is difficult to state a clear regulatory pathway, since all experiments did not show a strong $\alpha$-IgM-mediated suppression of the LEF1 gene (Figure 3-9 C). Additionally, basal LEF1 expression levels were upregulated upon most inhibitors used 
here. Nevertheless, an effect of the PI3K inhibitors Compound15e and BKM120 as well as the Mycinhibitor 10058-F4 can be postulated, suggesting a positive regulation of the LEF1 mRNA expression by PI3-kinase and c-Myc.

Overall, one can conclude that the BCR induced down regulation of all three genes is mediated via the PI3K-PLCY-PKC signaling pathway. Although slight differences in the responsiveness to the different inhibitors are observable, the results presented here point towards a joint regulation of PTCH1, MYC and LEF1 mRNA expression after BCR activation. 


\subsection{Analyzing intersections in signaling pathways via use of specific} chemical inhibitors

Microenvironmental factors are a dominant source of influence for most B cell lymphomas. It is important to know and to understand signaling modules and their effectors, which are involved in the tumor-micromilieu interactions. It is likely, that several immune response associated signals, such as CD40 and BCR induced signaling events, are specific mediators in B cell transformation. Thus, our group was able to mimic oncogenic pathway activities in aNHL by investigating the major patterns of gene expression changes in response to different stimuli in vitro and to subsequently identify individual aNHL profiles reflecting those patterns (Schrader et al., 2012b).

We therefore asked whether we could get access to comprehensive insights into potential targets for future individually designed therapy, based on gene expression profiles of aggressive nonHodgkin lymphoma. Thus, in this study a combination of in vitro stimulation with kinase inhibitors was used to delineate respective pathway interactions.

CD40 and $\alpha$-IgM activated signaling involves both similar and diverging pathways. However, even similar pathways are activated in quantitative different manners (compare Figure 3-1). To investigate the branching points in this complex signaling network, several kinases regulating different parts of specific signaling pathways were targeted to interrupt CD40 and $\alpha$-IgM mediated signaling and to determine subsequent changes in the gene expression profile.

As a first attempt, we meant to silence several transcription factors being crucial for signaling transduction on the gene expression level. We thought of targeting Bcl-6, c-Myc, Stat3 and p65 by RNAi mediated silencing. Unfortunately, previous work in our group showed strong difficulties to obtain differential gene expression profiles upon siRNA-mediated knock down of specific key players in diverging signaling pathways (unpublished data from K. Matulewicz). Even though an effective knock down was achievable in our model system, the Burkitt's lymphoma cell line BL2, we did not succeed to obtain comprehensive lists of specifically regulated genes. Although we could observe functional abrogation manifested in reduced proliferation or elevated apoptosis rates, current mathematical models did not succeed to exhibit differentially expressed genes. Controversially, we could even detect signaling pathway interruption on the protein level, but still the induced changes in gene expression were not strong enough to obtain lists of significant differentially expressed genes. Due to these complications, we decided to use chemical inhibitors instead of siRNA-mediated knock down. The major benefit of small molecule inhibitors is the active inhibition of enzymatic function, without inducing secondary effect by influencing the translational machinery. Another benefit of chemical (especially small molecule) inhibitors is the fast mode of action. When using 
RNAi, we had to transfect cells twice or even three-times and waited for at least 24 hours to observe effects. With the use of inhibitors, changes in signal transduction were rapidly detectable. When using inhibitors of specific kinases, we were able to detect changes in following protein phosphorylation as early as 5 minutes after treatment (data not shown). Additionally we used the advantage of targeting kinases instead of transcription factors to intervene on different levels of the signaling tree. Thereby, we aimed to dissect jointly regulated and branching points of the signaling network induced through CD40L or $\alpha$-IgM stimulation.

\subsubsection{Inhibitors in use}

To target kinases involved in both CD40 and BCR mediated signaling modules as well as kinases being specifically unique to BCR mediated signaling, we decided to target 6 different kinases in a way that we could perturb nearly the complete pathway and with use of a second and third inhibitor affect a single downstream effector. Thus, the use of one inhibitor aimed to directly affect the targets of one kinase, but the combination of several inhibitors affect the same targets as another inhibitor, targeting a more upstream kinase. Thereby, we were affecting only its direct targets, which are a subset of the phenotype obtained by blocking the complete pathway. A set of 6 different small molecule inhibitors were chosen based on literature and pretests in the group of $D$. Kube. We tried to choose inhibitors being already enrolled in clinical studies or having been described in research focused on hematological malignancies, mainly lymphomas.

The recombinant soluble $\mathrm{CD} 40 \mathrm{~L}$, used to stimulate transformed germinal center B cells in this study, mimics the CD40L/CD154, that is expressed either at the surface of or as soluble form by $C D 4^{+}$ T-helper cells, and activates its specific receptor CD40 (Aversa et al., 1993; Gascan et al., 1991; Hermann et al., 1993; Lane et al., 1992; Spriggs et al., 1992). CD40 mediated signaling mainly involves MAPK signaling via JNK and p38 and IKK activated canonical and non-canonical NF-KB signaling. The MAPKKK family member transforming growth factor-beta-activated kinase 1 (TAK1) has been reported to function as an 'upstream' molecule in IL-1R-mediated NF-KB and MAPK signaling pathways (Yamaguchi et al., 1995). Furthermore, Tak1 can be activated through latent membrane protein 1 from Epstein-Barr virus (Wan et al., 2004), which in turn is mimicking CD40 (Eliopoulos et al., 1996) and plays a pivotal role in EBV-positive Burkitt's lymphomas.

Antigen recognition through the $B$ cell receptor activates protein tyrosine kinases as for example Syk, which functions as amplifier of the BCR signal and connects the BCR with multiple signaling pathways, such as PI3K/Akt (Pogue et al., 2000). Furthermore, formation of the CBM (CARMA1/BCL10/MALT1) complex triggers the activation of NF-KB through IKK linkage (Egawa et al., 2003; Gaide et al., 2002; Hara et al., 2003; Jun and Goodnow, 2003; Newton and Dixit, 2003; RuefliBrasse et al., 2003; Ruland et al., 2001, 2003). Additionally, it has been proposed that Tak1 facilitates 
the IKK activation in response to BCR activation (Schuman et al., 2009; Shinohara and Kurosaki, 2009).

Likewise, B cell receptor crosslink also activates the MAPK signaling pathway by engagement of the Ras-Raf-mitogen-activated protein kinase/extracellular signal-regulated kinase kinase (MEK)extracellular signal-regulated kinase (ERK) mitogen-activated protein (MAP) kinase pathway (DeFranco, 1997; Richards et al., 1996, 2001), thereby affecting MEK1/2, p38 and JNK.

Thus we chose to target TAK1 as a key diversifier of signaling in both CD40 and BCR mediated signaling pathways and to treat our model cell line of transformed germinal center B cells with 100 nM (5Z)-7-Oxozeanol (Arcipowski and Bishop, 2012; Ear et al., 2010; Ninomiya-Tsuji et al., 2003; Schrader et al., 2012b). The inhibitor was tested in BL2 cells for pathway interruption and a diminished phosphorylation of p38 upon CD40 and BCR activation could be proven by Western Blot analysis (Figure 3-10 B).

NF-KB signaling was attenuated by inhibiting IKB $\alpha$ kinase (IKK) through $7 \mu \mathrm{M}$ IKK2 VIII-inhibitor (Murata et al., 2003). IKK is a large NF-KB-activating signaling complex, consisting of IKK $\alpha$ (IKK1), IKK $\beta$ (IKK2) and IKKY-NEMO, whereby IKK1 and IKK2 serve as catalytic subunits phosphorylating IKB $\alpha$, thereby promoting its degradation. The IKK2 inhibitor has been shown to abrogate phosphorylation of $\mathrm{I} \mathrm{KB} \alpha$ and NF-KB-DNA-binding as well as expression of NF-KB target genes leading to apoptosis in myeloma cells (Sanda et al., 2005). The inhibition of CD40 mediated NF-KB activation through IKK2 in transformed germinal center B cells was circumstantiated in OCI Ly1 cells. Nuclear translocation of p65 as activation characteristic for NF-KB signaling was abrogated upon inhibition with IKK2 inhibitor (Figure 3-10 D).

2 4M SB203580 was used to inhibit p38 activity (Craxton et al., 1998; Cuenda et al., 1995; Horie et al., 2007). This compound inhibits the activation of MAPKAP-2 by p38 and subsequent phosphorylation of HSP-27 (Cuenda et al., 1995). The efficiency of SB203580 has been shown before in pre-tests by other members in the group of $D$. Kube. Due to non-working antibody solutions a proof of principle by showing the inhibition of phosphorylation of HSP-27 through SB203580 in BL cells was not successful. Nonetheless, the specificity of this compound inhibiting p38 MAPK has been reported in several studies, including BL cell lines too (Birkenkamp et al., 2000; Cuenda et al., 1995; Fahmi et al., 2000; Horie et al., 2007; Schrantz et al., 2001; Vega et al., 2005; Vockerodt et al., 2001). To inhibit JNK activity $10 \mu \mathrm{M}$ SP600125 were used as reported from other studies in B cells (Bennett et al., 2001; Ke et al., 2006; Nishimura et al., 2009; Schnidar et al., 2009; Wang et al., 2009). This compound was a recommendation from A. Kieser (Munich GER). In his lab, JNK kinase assays were 
accomplished on lymphoblastoid cell lines (LCLs) to investigate the effect of SP600125 (Kutz et al., 2008).

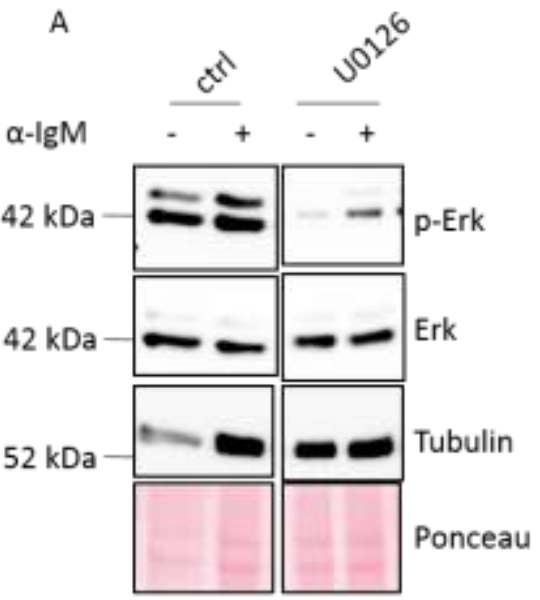

B
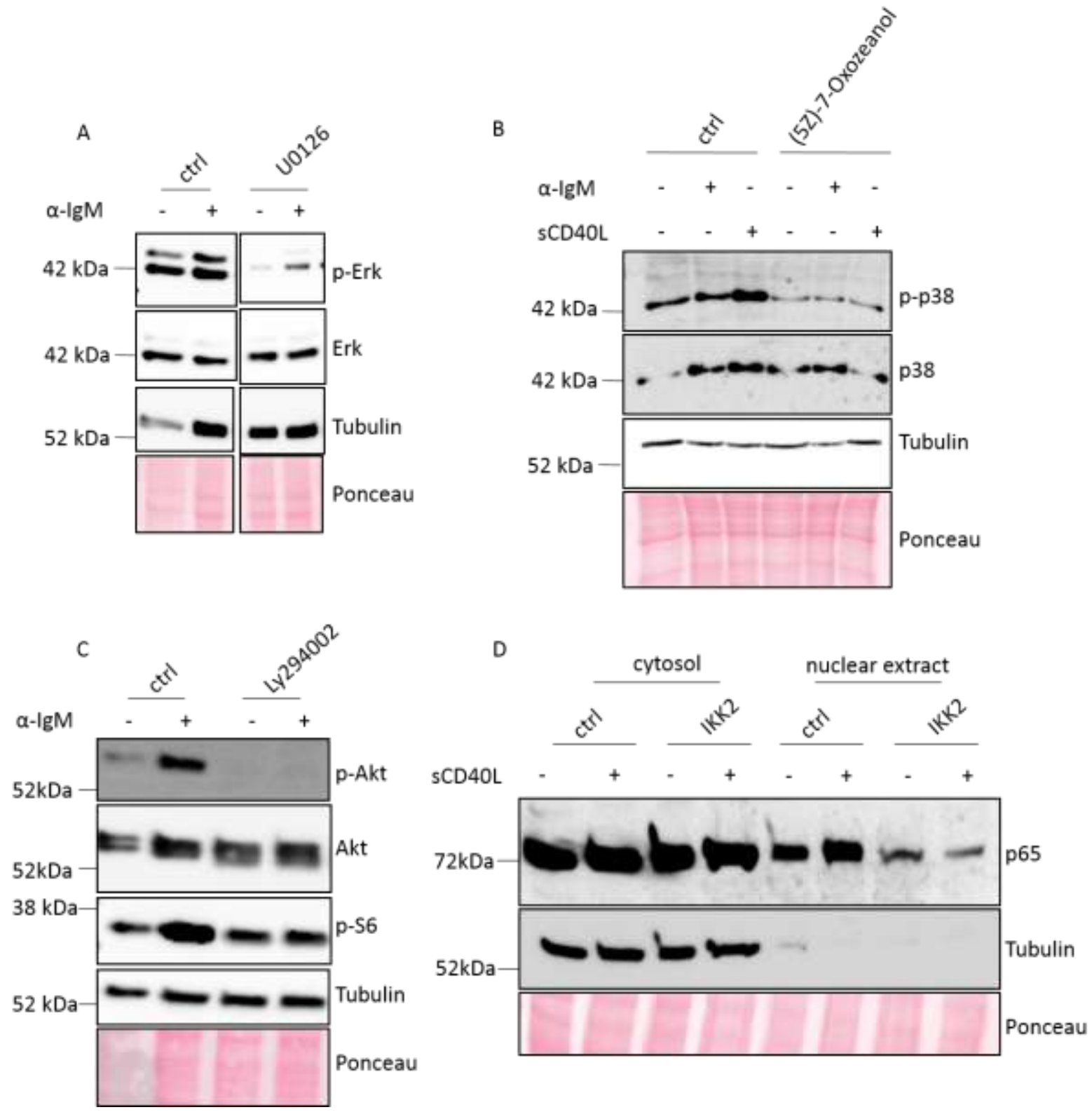

Figure 3-10 Proof of function of the inhibitors in use. BL2 cells were treated for 3 hours with respective inhibitors and subsequently 30 min stimulated with either $\alpha$-IgM antibody or SCD40L. Samples were subjected to Western Blot analysis to detect pathway inhibition upon inhibitor use. A U0126 treatment inhibits MEK1/2 activity upon BCR crosslink. Cells were treated with $10 \mu \mathrm{M}$ U0126 or dmso (ctrl) and inhibition of MEK1/2 was detected by diminished phosphorylation of Erk through phospho-specific antibodies detecting p-p42/p44. B The Tak1 inhibitor (5Z)-7-Oxozeanol reduces p-p38 levels in BL2 cells. Cells were treated with $100 \mathrm{nM}(5 \mathrm{Z})-7-$ Oxozeanol and phosphorylation of p38 was detected using specific antibodies. C $10 \mu \mathrm{M}$ of the PI3K-inhibitor Ly294002 were used to inhibit BCR induced signaling, detected here by inhibited phosphorylation of Akt and S6. D IKK2 inhibitor ACHP prevents NF-KB activation. OCI Ly1 cells were pre-treated for 3 hours with $14 \mu \mathrm{M}$ IKK2 inhibitor (ACHP) and subsequently CD40 signaling was activated via SCD40L. Nuclear extracts were analyzed for p65 translocation in the nucleus to monitor NF-KB activation. 
To specifically dissect BCR mediated signaling modules, additionally PI3K and MEK1/2 activity was inhibited using 10 M Ly294002 (PI3K) (Curnock and Knox, 1998; Uddin et al., 2006; Vlahos et al., 1994) and $10 \mu \mathrm{M}$ U0126 (MEK1/2) respectively (Favata et al., 1998; Zheng et al., 2003). BL2 cells were pre-incubated for 3 hours with respective concentrations of specific inhibitor to ensure a complete mode of action of the inhibitor. To demonstrate the efficacy of U0126 in BL2 cells, the phosphorylation status of Erk1/2 upon $\alpha$-IgM stimulation was monitored by WB using a phosphospecific antibody against p42/p44 (Erk1/2) (Figure 3-10 A). The activity of Ly294002 could be shown under similar conditions through abrogated phosphorylation of Akt upon inhibitor use as well as inhibition of phosphorylation of the downstream effector S6 (Figure 3-10 C).

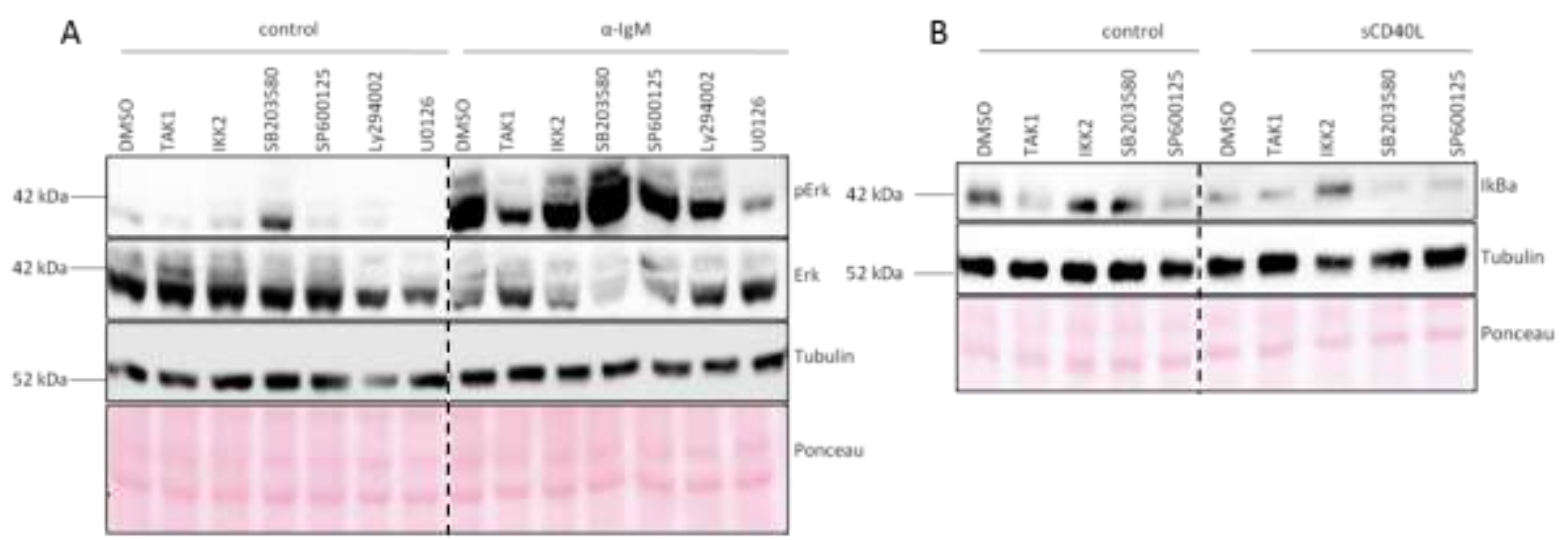

Figure 3-11 Effects of inhibitors used in microarray analyses. Western Blot analyses of 20 ng protein of samples of batch III, subjected to microarray hybridization, but harvested 30 min after stimulation to control successful activation of signaling pathways. Due to sample limitations, only one signaling pathway could have been analysed. A Samples subjected to $\alpha$-IgM stimulation were verified for active phosphorylation of Erk upon stimulation using specific antibodies. B Samples subjected to CD40 activation were counterchecked for active NF-KB signaling via degradation of I $\mathrm{B} \alpha$.

To verify the activity of the inhibitors and the successful stimulation of the BL cells in the specific samples dedicated for microarray hybridization, part of the samples were harvested upon 30 min stimulation to subject them to Western Blot analyses. As exemplified in Figure 3-11, the successful stimulation through $\alpha-\operatorname{lgM}$ antibody was verified via phosphorylation of Erk (Figure 3-11 A) and the stimulation through $\mathrm{SCD} 40 \mathrm{~L}$ was verified by degradation of IKB $\alpha$ as marker for active NF-KB signaling (Figure 3-11 B). Figure 3-11 A depicts a strong phosphorylation of Erk upon $\alpha$-IgM stimulation, which was inhibited by U0126. Additionally, a reduction in phosphorylation of Erk was observable after Tak1 inhibition (5Z-7-Oxozeanol). This was not expected, but may be supported by some few indirect hints proposing a connection between Tak1 and MEK1/Erk signaling (Ninomiya-Tsuji et al., 2003; Ohori et al., 2007; Schirmer et al., 2006). However, the effect of Tak1 inhibition on the phosphorylation status of Erk has not been shown before to our knowledge. Similar to Birkenkamp and coworkers in AML we observed an induction of phosphorylation of Erk upon p38 kinase inhibition through the use of SB203580 (Birkenkamp et al., 2000). CD40 activation led in turn to 
active NF-KB signaling visualized by the degradation of IKB $\alpha$ (Figure 3-11 B). IKK2 inhibition abrogated the degradation of $1 \mathrm{~KB} \alpha$ as expected. No activation of NF-KB signaling by inhibition of $\mathrm{p} 38$ or JNK (SB203580 or SP600125 respectively) was detected. The activation of NF-KB by the Tak1 inhibitor (5Z)-7-Oxozeanol was visible only once in all experiments conducted. Therefore we interpreted this as a technical artefact (compare Figure 3-13 A and Figure 3-14).

To conclude, the herein presented protein studies demonstrate that the chosen inhibitors interrupt the distinct signaling pathways they are aiming at. Thus we assume effects on the gene expression profile upon use of those inhibitors.

\subsubsection{Differential gene expression on the whole genome level upon inhibitor use}

To investigate the gene expression changes that are induced through specific pathway interruption under stimulation in a global way, gene expression profiling was performed. Samples of 3 independent biological replicates were hybridized to Affymetrix HG U133 Plus2.0 GeneChip arrays. Data were analysed with support from K. Meyer, M. Pirkl and Prof. Dr. R. Spang from the Institute of Functional Genomics in Regensburg. Differentially expressed genes were identified using linear models as implemented in the Bioconductor R package LIMMA (Smyth et al., 2005). Genes which show a significant (adj. p-value $\geq 0.05$ ) change in expression were considered.

The stimulation of BL2 cells through BCR cross linking led to the differential expression of 16540 probes, mapping 9044 genes. CD40 stimulation of BL2 cells led to differential expression of 54654 probes, which are mapping 2634 genes. Due to the high number of significantly differentially expressed genes, in the following expression changes were sorted according to their absolute logarithmic fold changes $(\log F C)$ and only probe sets displaying $\log F C \geq 0.75$ or $\log F C \leq-0.75$ were taken into consideration. This process drops all genes with an expression less than 1.5 -fold $(0.5$-fold respectively) of the control value.

The here conducted study comprises a large amount of comprehensive data. The numbers of differentially expressed genes upon each comparison between inhibitor, combination of inhibitors and stimulation are summarized in Table 3-1. A complete list of differentially expressed genes after BCR crosslink and CD40 stimulation can be found on the externally provided CD ROM (\\NEM\LimmaLists\). 
Table 3-1 Differential expression in human transformed germinal center B cells in response to inhibition of B cell specific stimulations. Summarized are the numbers of differentially expressed genes in response to each inhibitor in comparison to unstimulated BL2 cells or BL2 cells stimulated for 3 hours with $\alpha$-lgM antibody or sCD40L. The total number of affected genes comprises all genes significantly differentially expressed (adj. $p$ value $\leq 0.05$ ) and is computed out of affected probes represented on the HG U133 Plus 2.0 microarray. Thus several genes are represented through probes which are marginally up as well as down regulated, which explains why the sum of up and down regulated genes does not necessarily correspond to the total number of affected genes. Due to high amounts of affected genes with very small effective fold change, genes exposing a $\operatorname{logFC} \geq 0.75$ or $\log \mathrm{FC} \leq-075$ were regarded as differentially expressed.

\begin{tabular}{|c|c|c|c|}
\hline & $\begin{array}{l}\text { total \# of affected } \\
\text { genes (up/down) }\end{array}$ & $\begin{array}{l}\text { up regulated genes } \\
(\operatorname{logFC} \geq 0.75)\end{array}$ & $\begin{array}{l}\text { down regulated genes } \\
(\operatorname{logFC} \leq-0.75)\end{array}$ \\
\hline \multicolumn{4}{|l|}{ Inhibitor Vs Ctrl } \\
\hline Ly294002 (PI3K) & $3875(2025 / 1888)$ & 73 & 84 \\
\hline U0126 (MEK1/2) & $303(234 / 62)$ & 16 & 1 \\
\hline (5Z)-7-Oxozeanol (Tak1) & $20(20 / 2)$ & 0 & 1 \\
\hline ACHP (IKK2) & $4561(2139 / 2535)$ & 22 & 66 \\
\hline SB203580 (p38) & $21(18 / 3)$ & 3 & 0 \\
\hline SP600125 (JNK) & $1566(1175 / 397)$ & 130 & 6 \\
\hline IKK2/p38 & $4387(2153 / 2383)$ & 99 & 175 \\
\hline JNK/p38 & $4150(2188 / 2038)$ & 63 & 32 \\
\hline IKK2/JNK & $5804(2096 / 3087)$ & 168 & 160 \\
\hline IKK2/JNK/p38 & $4574(2609 / 2099)$ & 151 & 53 \\
\hline \multicolumn{4}{|l|}{$\alpha-\lg M$} \\
\hline ctrl & $9044(3548 / 5770)$ & 378 & 974 \\
\hline Ly294002 (PI3K) & $5871(4387 / 1558)$ & 409 & 127 \\
\hline U0126 (MEK1/2) & $6539(4834 / 1795)$ & 410 & 171 \\
\hline (5Z)-7-Oxozeanol (Tak1) & $407(167 / 241)$ & 15 & 30 \\
\hline ACHP (IKK2) & $2220(1342 / 900)$ & 56 & 60 \\
\hline SB203580 (p38) & $36(11 / 25)$ & 0 & 1 \\
\hline SP600125 (JNK) & $860(690 / 171)$ & 30 & 13 \\
\hline IKK2/p38 & 3477 (2320/1257) & 195 & 103 \\
\hline JNK/p38 & $2513(2114 / 410)$ & 44 & 15 \\
\hline IKK2/JNK & $4886(3373 / 1640)$ & 230 & 106 \\
\hline IKK2/JNK/p38 & $5512(3817 / 1867)$ & 365 & 139 \\
\hline \multicolumn{4}{|l|}{ sCD40L } \\
\hline ctrl & $2633(1352 / 1323)$ & 129 & 115 \\
\hline (5Z)-7-Oxozeanol (Tak1) & $1838(1001 / 862)$ & 79 & 85 \\
\hline ACHP (IKK2) & $4549(2232 / 2575)$ & 62 & 257 \\
\hline SB203580 (p38) & $3453(2137 / 1404)$ & 198 & 175 \\
\hline SP600125 (JNK) & 4589 (3051/1668) & 280 & 121 \\
\hline IKK2/p38 & $5870(3405 / 2929)$ & 120 & 382 \\
\hline JNK/p38 & $6028(3588 / 2789)$ & 289 & 390 \\
\hline IKK2/JNK & $5654(3305 / 2765)$ & 285 & 244 \\
\hline IKK2/JNK/p38 & $6113(3316 / 3326)$ & 239 & 373 \\
\hline
\end{tabular}


In the present study, small molecule inhibitors were used to abrogate the function of specific kinases in order to obtain gene expression changes upon signaling pathway activation through CD40L or BCR cross linking. To investigate the effect of the specific inhibitor on the stimulated BL cells and to be aware of potential side-effects or effects without activation of the respective pathway two different experimental procedures were analyzed for each inhibitor: (1) cells treated with inhibitor alone and (2) stimulated cells treated with inhibitor. So, for each inhibitor or combination of inhibitors 3 lists of differentially expressed genes were obtained: (a) INHIBITOR-CTRL (contains all genes differentially regulated upon inhibitor use in unstimulated BL2 cells); (b) INHIBITORxBCRCtrIBCR (shows all differentially expressed genes between BL2 cells incubated 3 hours with inhibitor and subsequently stimulated 3 hours with $\alpha$-IgM antibody and BL2 cells incubated 3 hours with ctrl (dmso) and subsequently stimulated 3 hours with $\alpha$-IgM antibody) and (c) INHIBITORxCD40-CtrICD40 (exhibits all differentially expressed genes between BL2 cells incubated 3 hours with inhibitor and subsequent CD40 activation and the respective control: untreated/dmso-treated cells stimulated with SCD40L).

The list of differentially expressed genes upon inhibitor use on unstimulated cells compared with the unstimulated control BL2 cells, gives a first overview about the effects of the inhibitor on the cell type of Burkitt's lymphoma cells. Those effects observed here may include unspecific actions of the inhibitor as well as the exertion of inhibitory effects on already activated signaling pathways in the normal turnover of BL2 cells.

As shown in Table 3-1, most of the inhibitors used in this study did not exert large effects on the unstimulated BL2 cells. Major effects upon inhibitor use on unstimulated BL2 cells were detected upon PI3K, IKK2 and JNK inhibition. This may point towards active basal signaling of PI3K, NF-KB and JNK in Burkitt's lymphoma cells. Supporting this, a "tonic BCR signal" including augmented activity of PI3 kinase in Burkitt's lymphoma (Schmitz et al., 2012) and a synergistic action of c-Myc and PI3K signaling in Burkitt's lymphomagenesis (Sander et al., 2012) have been described recently. Although BL typically do not exhibit constitutive active NF-KB signaling in contrast to other lymphoma entitites (Dave et al., 2006; Klapproth et al., 2009), several hints point towards a sustained NF-KB activity in BL2 cells (see also chapter 3.3.4). In addition, our group could recently show a tonic activation of PI3K, NF-KB and JNK to regulate aberrant 'basal' c-Myc expression in BL (Schrader et al., 2012b).

This dataset comprises a highly comprehensive amount of data. Therefore, no specific single differentially expressed genes will be considered, but network analyses provided by M. Pirkl, K. Meyer and Prof. Dr. R. Spang (university of Regensburg) will be presented in the next paragraphs. 


\subsubsection{Nested Effects Models propose a controversial role of Tak1 in CD40 and BCR mediated signaling.}

One of the main aims of the present study was to provide high-dimensional data to develop, establish and further improve computational methods. A key obstacle to infer signaling networks from perturbation screens is that gene expression profiles offer only an indirect view of pathway structure due to the high number of non-transcriptional regulatory events like protein modifications. A recent computational approach especially designed to learn from high-dimensional phenotypes and indirect information are Nested Effects Models (NEM) (Markowetz et al., 2005, 2007). NEMs are a class of probabilistic models, which reverse engineer upstream/downstream relations of cellular signaling cascades and are computed to return pathway structures explaining the perturbation effects.

The present study was designed in order to provide sufficient perturbations as well as upstream/downstream relations to infer signaling pathway structures with the use of NEMs.

As described above, CD40L and $\alpha$-IgM antibody induce overlapping as well as specific signaling pathways in BL2 cells. Based on the high-dimensional gene expression data, consolidating several different perturbations in terms of pathway activation via stimulation and pathway interruption via kinase inhibition, nested effects models were generated returning pathway structures analyzing and comparing the role of Tak1 in CD40 and BCR mediated signaling. Prior knowledge based on literature discussed Tak1 as signal diversifier upstream of p38, NF-KB and JNK activation in CD40 activated signaling (Sato et al., 2005; Takaesu et al., 2003; Yamashita et al., 2008). Equally, an essential role as mediator for BCR induced NF-KB signaling has been reported for Tak1 (Schuman et al., 2009; Shinohara and Kurosaki, 2009). Additionally, a very recent report being published throughout the work on the present study demonstrates a pivotal role for Tak1 in regulating cell survival of mantle cell lymphoma, a lymphoma subtype being dependent on active NF-kB signaling (Buglio et al., 2012). However, the role of Tak1 in NF-KB-independent malignancies as Burkitt's lymphoma remains to be elucidated so far.

As shown in Figure 3-12, NEMs derived two independent pathway structures for CD40 and BCR mediated signaling, proposing individual roles for Tak1. The firstly provided nested effects models supposed a covalent activation of p38 and Tak1 both exerting effects on the activity of IKK2 (Figure 3-12 A). Furthermore, computed modeling resulted in an interchangeability of IKK2 and JNK, due to a strong overlap of target genes, when perturbing both kinases. However, Tak1 did not present as mediator that integrates signaling crosstalks. A similar surprising observation was made for BCR mediated signaling. Here, PI3K presented as expected as upstream signaling modifier. Nevertheless, Tak1 could not be modeled to be downstream of PI3K and upstream of NF-KB activation as already 
described in literature (Schuman et al., 2009; Shinohara et al., 2005). Instead, p38, JNK and NF-KB signaling presented to be completely independent of Tak1 kinase function, but vice versa exerting effects on the shared target Erk1/2. The results proposed by the computed nested effects models were completely surprising, unexpected and contradictory to recent reports in other cell types, thus a biochemical proof was needed and several further experiments were conducted.

A

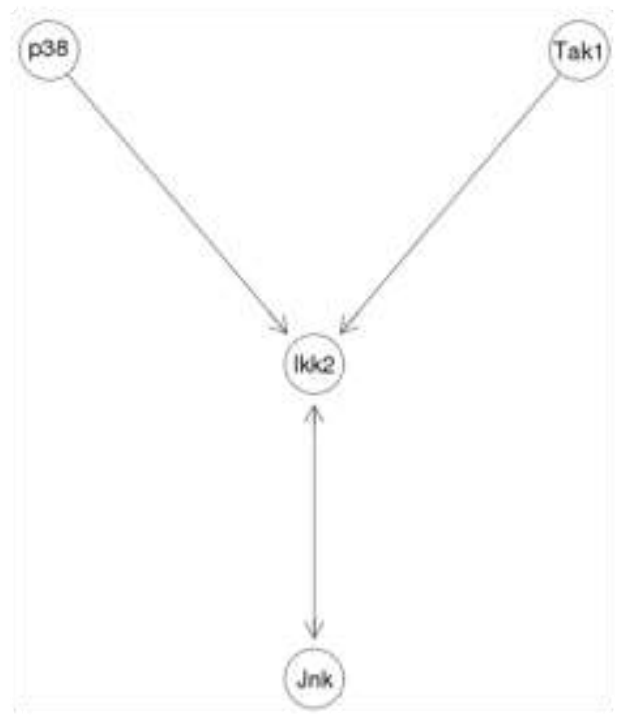

B

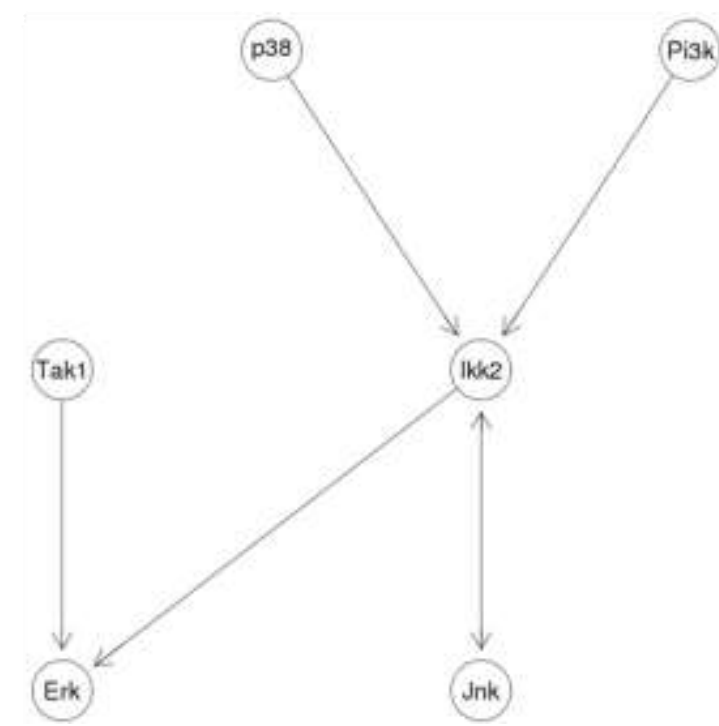

Figure 3-12 Role of Tak1 in CD40 and BCR mediated signaling proposed by a first generation Nested Effects

Model. A Nested Effects Model (NEM) of CD40 mediated signaling suggests an influence of Tak1 on IKK2 and JNk, but the p38-excerted effect seems to be independent of Tak1. Furthermore are IKK2 inhibition and Jnk inhibition indistinguishable with regard to their gene expression profile. B NEM of BCR mediated signaling. p38, NF-kB and JNK signaling seem to be completely independent from Tak1 signaling, but PI3K inhibition leads to effects on NF-kB signaling. Additionally, Tak1 inhibition is proposed to be connected to Erk-pathway by sharing target genes.

To exclude probabilistic side effects of the used chemical inhibitor (5Z)-7-Oxozeanol, one additional small molecule inhibitor of Tak1 (AZ-TAK, Tocris, Bristol UK) and siRNA targeted against Tak1 was used in a comparative study (Figure 3-13). BL2 cells were transfected twice with a time lapse of 24 hours with control-siRNA (scrbl) or siRNA targeted against Tak1 (Silencer ${ }^{\circledR}$ Select, Ambion), respectively. Control-siRNA transfected cells were subsequently splitted and additionally pre-treated with (5Z)-7-Oxozeanol or AZ-TAK1 for 3 hours, before all cells were either stimulated with $\mathrm{SCD} 40 \mathrm{~L}$ or $\alpha-\operatorname{IgM}$ antibody or left unstimulated. Stimulation was carried out for $30 \mathrm{~min}$ to analyze pathway activation signals via Western Blot analyses or for 3 hours respectively to analyzing target gene expression changes via qRT-PCR. In the following the activation of MAPK signaling (p38, Erk) and NF-KB signaling (IKB $\alpha$ degradation) was investigated using Western Blot analyses. JNK signaling was not contemplated due to the complexity of the here considered data, and non-working antibodies for phosphorylated JNK levels. 
As clearly shown in Figure 3-13 A, (5Z)-7-Oxozeanol strongly inhibited CD40 and BCR induced phosphorylation of p38. Thus, Tak1 should be presented upstream of p38 both in CD40 as well as in $\mathrm{BCR}$ activated signaling models. Additionally, protein analyses of IKB $\alpha$-levels in Tak1-inhibitor treated BL2 cells revealed a stabilization of IKB $\alpha$ upon inhibitor use and subsequent CD40 activation. In control-treated cells a marked degradation of IKB $\alpha$ after CD40 activation as hint for active NF-KB signaling was visible. BCR crosslink did not induce strong NF-KB activation, observable as IKB $\alpha$ degradation, in BL2 cells, therefore the effect of the inhibitor on BCR induced NF-KB signaling remains unclear upon analyses of protein modifications. The inhibitor AZ-TAK obtained from Tocris Bioscience (Bristol UK) did not seem to work properly in our hands. Buglio and coworkers could demonstrate reduced phosphorylation levels of p38 in an IL-1-induced HeLa cell line upon AZ-TAK1 treatment for 1 hour (Buglio et al., 2012), but here the phosphorylation status of p38 was already elevated upon AZ-TAK1 treatment of unstimulated BL2 cells compared to untreated BL2 cells. Same holds true for the use of siRNA mediated knock down of the gene of interest.

A strong reduction in protein levels of Tak1 was observable upon siRNA use, but the activation of p38 signaling through CD4OL is still present. Similarly was the inhibition of NF-KB signaling, as visualized by IKB $\alpha$ degradation, most clearly detectable upon use of (5Z)-7-Oxozeanol and less convincing (but still observable) upon use of AZ-TAK or TAK1-targeting siRNA.

As already mentioned above the read out for BCR activated signaling on the protein level is more difficult. The p38 activation upon 30 min $\alpha$-IgM stimulation was not as strong as after CD40L, but was nevertheless inhibited through (5Z)-7-Oxozeanol. IKB $\alpha$ degradation was not observable after $30 \mathrm{~min}$ of $\mathrm{BCR}$ crosslink, but a strong activation of Erk signaling was detectable via phosphorylation of Erk1/2. Upon use of Tak1 inhibitors and siRNA targeted against TAK1, the phosphorylation status of Erk1/2 seemed to be reduced.

Nevertheless, those analyses of protein modifications upon Tak1 inhibition and subsequent pathway activation led to the conclusion that Tak1 should be modeled as a signal modulator being situated upstream of p38 and NF-KB signals in CD40 mediated signaling as well as upstream of p38, NF-KB and Erk signals in the BCR pathway. 
A

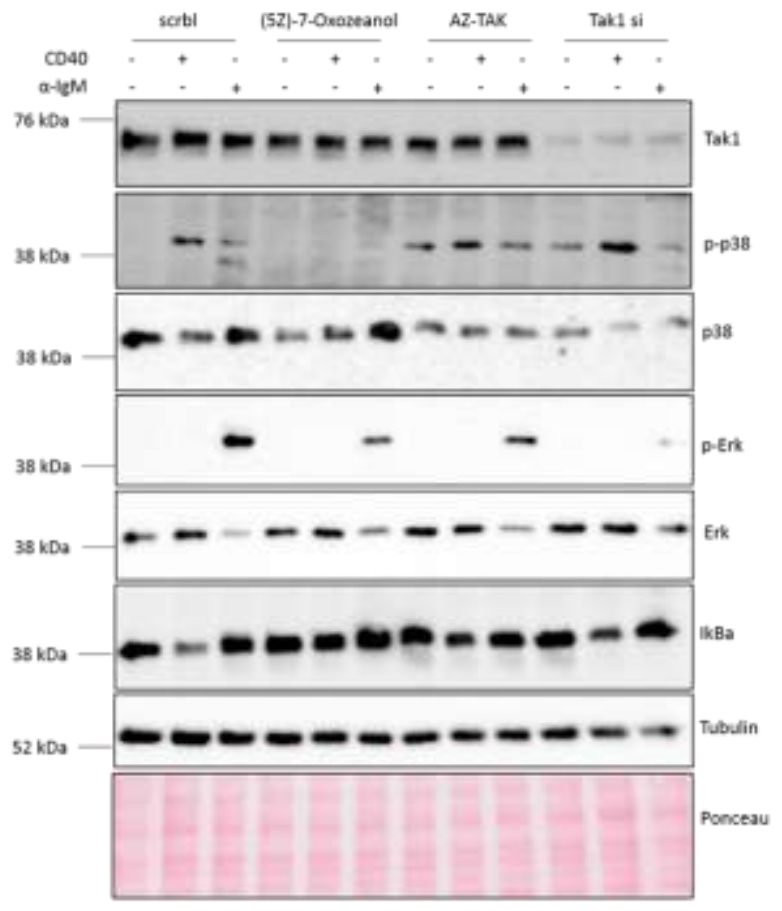

E
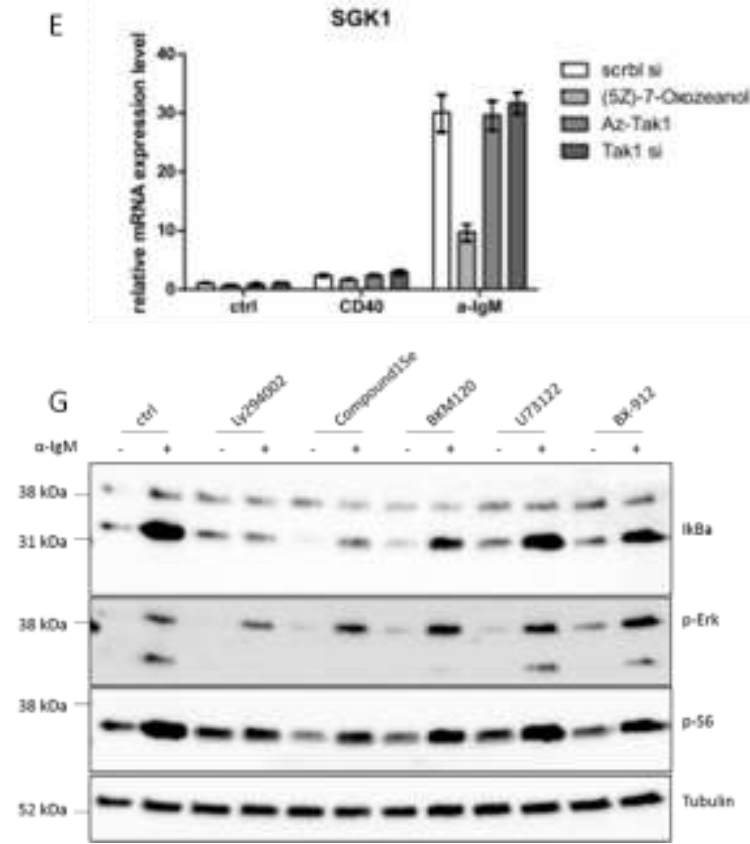

B

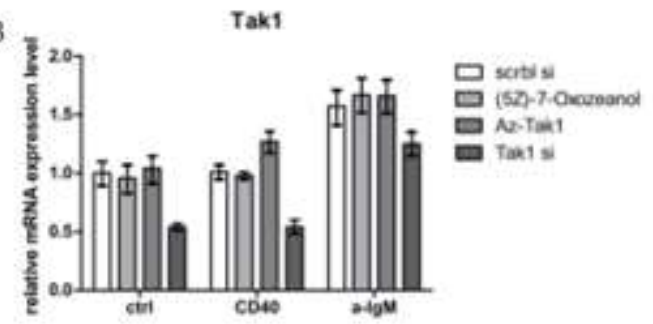

C

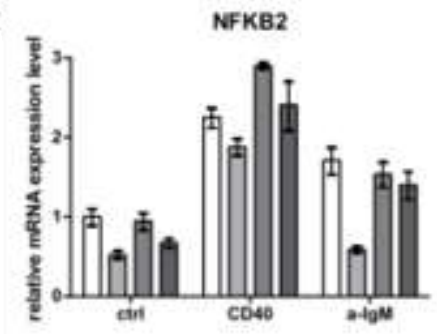

D

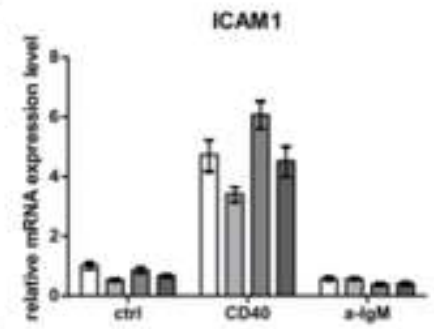

LINC00158

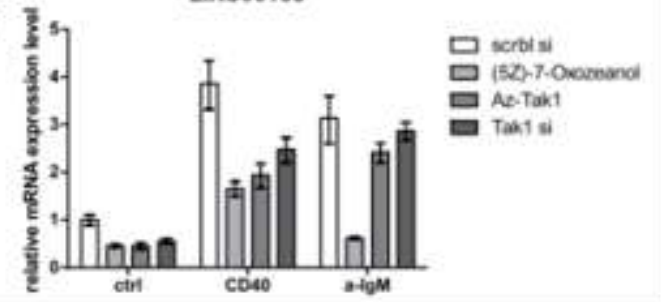

।
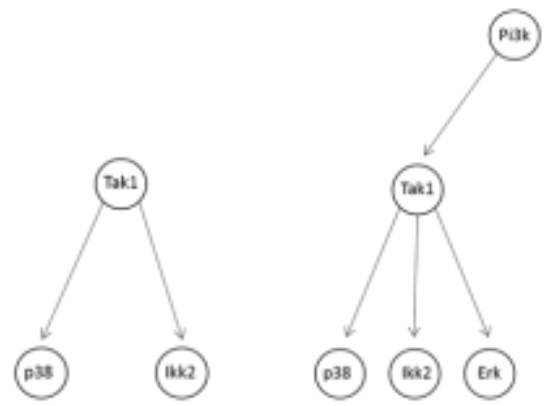

Figure 3-13 Tak1 presents as major signal modulator in CD40 and BCR mediated signaling. A Western Blot analysis of BL2 cells transfected twice for 24 hours with $4 \mu \mathrm{g}$ scrbl siRNA or Tak1 siRNA. Samples transfected with scrbl siRNA were splitted 24 hours after second transfection for treatment with either $100 \mathrm{nM}$ (5Z)-7-

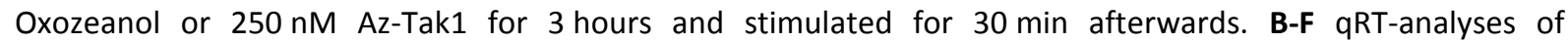
transfected BL2 cells (same treatment as in A, apart from 3 hours of stimulation with either SCD40L or $\alpha$-IgM $\mathrm{F}(\mathrm{ab})_{2}$ fragment). Data are represented as $2^{-\Delta \Delta \mathrm{Ct}}$ values relative to $A B L$ housekeeping gene expression. $\mathbf{G}$ Western Blot analysis of several inhibitors of BCR mediated signaling in BL2 cells: PI3K (Ly294002, Compund15e, BKM120), PLC- $\nu$ (U73122), PDK1 (BX-912). H Model of CD40 mediated signaling based on experimental data involves Tak1 in p38 and NF-KB signaling. I Model of BCR mediated signaling based on 
experimental data places Tak1 downstream of PI3K, but upstream of p38, NF-KB and Erk-signaling. The role of JNK in this context was not determined.

To further elucidate gene expression changes induced through Tak1 inhibition, qRT-PCR analyses were accomplished using the same experimental procedure as described above, except 3 hours of CD40/BCR stimulation before analysis. First of all, TAK1 mRNA expression level were checked to prove a knock down of TAK1 upon siRNA use. The results showed $50 \%$ reduced expression levels upon siRNA, but not upon inhibitor use, as the chemicals inhibit kinase activity and not protein expression (Figure 3-13 A \& B). To further investigate the NF-KB activation through BCR crosslink typical NF-KB target genes as NFKB2 and ICAM1 have been analyzed. One can observe an induction of NFKB2 expression upon CD40 stimulation, which is much less pronounced upon BCR crosslink (Figure 3-13 C). However, (5Z)-7-Oxozeanol, but not AZ-TAK1 or Tak1 siRNA reduced the levels of NFKB2 mRNA expression. Such a reduction in NFKB2 expression level was even in the unstimulated BL2 cells detectable. Furthermore, the expression of ICAM1 was markedly elevated upon CD40 activation, but not changed after $\alpha$-IgM stimulation (Figure 3-13 D). The level of ICAM1 activation was similarly suppressed through (5Z)-7-Oxozeanol treatment after pathway activation through CD4OL as in unstimulated BL 2 cells. Figure 3-13 E \& F shows the regulation of mRNA expression upon Tak1 inhibition and subsequent CD40/BCR stimulation of SGK1 and LINCO0158. SGK1, the serum- and glucocorticoid-induced kinase 1, is activated through the PI3K (Yan et al., 2008), thus SGK1 mRNA expression level are highly up regulated upon $\alpha$-IgM stimulation (Schrader et al., 2012b). LINC00158 is a long intergenic non-protein coding RNA 158, which was found to be strongly regulated through CD40 and BCR activation (Schrader et al., 2012b) as well as IKK2 and Tak1 inhibition in CD40 activated BL cells (compare supplemental file IINEM\combinedAnalyses_201211\IKK2_Tak1xCD40.int.xls on the provided CD ROM). For both genes a strong inhibition through the use of (5Z)-7-Oxozeanol was detectable, which confirms the microarray data by qRT-PCR and contradicts the proposed nested effects model for BCR. Additionally, similar to the NF-KB target genes NFKB2 and ICAM1, a regulation by Tak1 inhibition in unstimulated BL2 cells for LINC00158 was observed.

To further verify the proposed NEM for BCR activated signaling, the role of PI3K signaling in regard to its possibility to activate NF-KB signaling in BL cells was investigated. Several small molecule inhibitors targeting BCR induced signaling on different levels were used. Thus, BL2 cells were 3 hours pre-treated with Ly294002, Compound15e or BKM120 to inhibit PI3K activity with three independent inhibitors, as well as with inhibitors for PLC- $\gamma$ (U73122) and PDK1 (BX-912) before subsequent stimulation with $\alpha$-lgM antibody for 60 min (Kopp et al., 2012; Mogami et al., 1997; Vlahos et al., 1994; Walsh et al., 2013). As depicted in Figure 3-13 G, we could not detect a BCR induced degradation of IKB $\alpha$ in BL2 cells. Upon inhibition of basal ('tonic') and BCR induced PI3K activity via Ly294002 and Compound15e the stabilization of IкB $\alpha$ was abolished. Although not abolished, a 
diminished stabilization of IKB $\alpha$ was also visible upon use of BX-912. The here demonstrated results raise the question if basal PI3K activity, as observed in BL (Schmitz et al., 2012), restrains NF-KB activatability. Therefore, one can conclude that NF-KB activity is also regulated through $\mathrm{PI} 3 \mathrm{~K}$ activation in BCR signaling. Similarly, a strong reduction in BCR induced phosphorylation levels of Erk were observed after PI3K-inhibition.

Summarizing the data presented in Figure $3-13$, one can conclude that in CD40 mediated signaling Tak1 may indeed influence p38 and NF-KB signaling as upstream mediator, whereas in BCR mediated signaling Tak1 additionally modulates Erk signaling and may be interposed between PI3K influence upstream and p38, Erk and NF-KB signaling downstream (Figure 3-13 H \& I). To further pinpoint the role of Tak1 in CD40 and BCR mediated signaling in BL cells, the experimental procedure as described above was expanded on another Burkitt's lymphoma cell line Ramos (Figure 3-14 B), thereby disclosing some particularities. In BL2 cells, p38 is activated upon both CD4OL and $\alpha$-IgM stimulation. (5Z)-7-Oxozeanol inhibited this activation markedly, whereas upon AZ-TAK treatment only diminished phosphorylation levels of p38 were detectable and the use of Tak1 siRNA did not affect the activation status of p38 (Figure 3-14 A). In Ramos cells, the p38 activation strongly appeared after CD40 pathway activation, but not upon BCR crosslink. Nevertheless, the effect of (5Z)-7-Oxozeanol was much stronger in Ramos cells than in BL2 cells, whereas the effect of AZ-TAK and Tak1 siRNA were comparable in both cell lines (Figure 3-14 B).
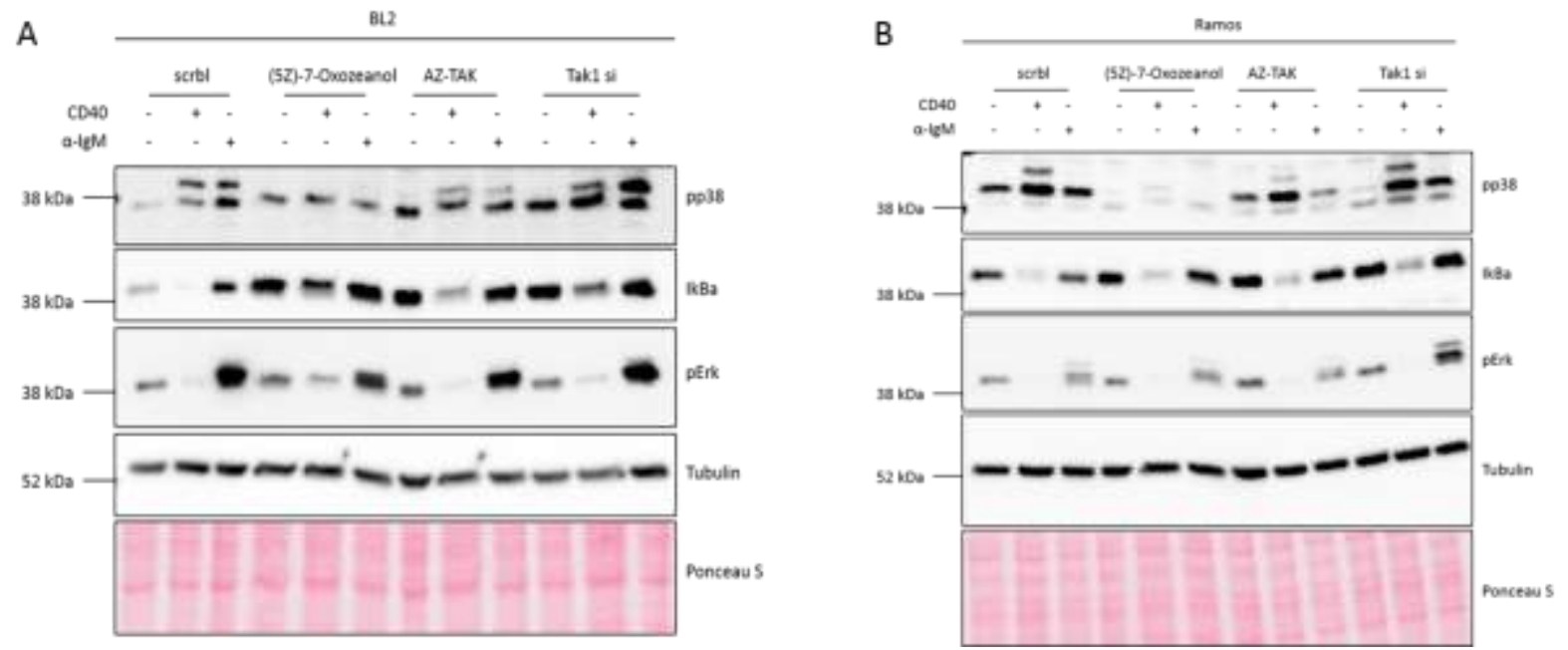

Figure 3-14 Comparison of two Burkitt's lymphoma cell lines with regard to signaling interruption upon Tak1 inhibition. A BL2 cells were transfected with Tak1 siRNA or respective control. Control transfected cells were splitted and additionally treated with Tak1 inhibitors (5Z)-7-Oxozeanol or AZ-TAK. After 3 hours of inhibitor treatment, cells were stimulated for 30 min with either SCD40L or $\alpha-\operatorname{lgM~F}(a b)_{2}$ fragment or left as control. Samples were harvested and $20 \mu \mathrm{g}$ protein were subjected to Western Blot analyses. B The Burkitt's lymphoma cell line Ramos was manipulated as in $\mathbf{A}$. Shown is one representative result of at least 3 replicates. 
NF-KB signaling was in both cell lines strongly activated upon CD40 activation and nearly not detectable via WB after 30 min BCR crosslink. In BL2 cells, (5Z)-7-Oxozeanol inhibited the NF-KB activation, whereas in Ramos cells still a strong ІкBa degradation was detectable. Both AZ-TAK as well as Tak1-directed siRNA marginally led to an IKBa stabilization in BL2 as well as in Ramos cells.

Furthermore, the activation of the Erk pathway after BCR crosslink, displayed here through phosphorylation of Erk was much stronger in BL2 cells than in Ramos cells, thus the inhibitory effect of (5Z)-7-Oxozeanol is much easier to demonstrate in BL2 cells than in Ramos cells. All these observations point to a somehow particular role of BL2 cells, our model cell line for human transformed germinal center cells. Preliminary results from others in the group of D. Kube investigating further Burkitt's lymphoma cell lines, tend to confirm an outstanding role of the BL2 cell line in the scope of Burkitt's lymphoma cell lines. Nevertheless, the data used to compute nested effects models were obtained on perturbations of the BL2 cell line and in this cell line Tak1 seems to play its foretold role as mediator of p38 and NF-KB signaling upon CD40 activation as well as diversifier of signaling towards p38, NF-KB and Erk signaling after BCR crosslink.

Taken together, the biochemically collected data provide strong evidence that the primarily proposed nested effects models of the role of Tak1 in CD40 and BCR mediated signaling are inadequately describing the underlying signaling functions of Tak1. Thus, further computational advances have to be made to correctly model the here presented gene expression data. 


\subsubsection{NF-KB signaling upon BCR crosslink in Burkitt's lymphoma}

Simultaneously to the question whether Tak1 plays its pivotal role as mediator for NF-kB signaling in BLs as well upon BCR signaling, the observation of generally low levels of NF-KB activation after $\alpha$ IgM stimulation in BL cells was investigated. Generally, triggering of the B cell receptor leads to the activation of NF-KB signaling via protein kinase C (PKC) $\beta$ and additional signal adaptors like CARMA1, Bcl10 and mucosal-associated lymphoid tissue 1 (MALT1) (Egawa et al., 2003; Li and Verma, 2002; Ruefli-Brasse et al., 2003; Ruland and Mak, 2003).

Interestingly, the results reported in the present study exhibit the contrasting observation of a reduced activatability of NF-KB signaling through $\mathrm{B}$ cell receptor signaling compared to CD40 mediated signaling in the BL cell lines BL2 and Ramos (compare Figure 3-14). Moreover, taking IKB $\alpha$ degradation as a sign for NF-KB activation, the impression of reduced NF-KB signaling after BCR activation arises, as visualized by a sustained stabilization of IкB $\alpha$ (Figure 3-13 G and Figure 3-14 A).

To elucidate the reduced NF-KB signaling upon BCR crosslink in Burkitt's lymphoma, the different NF-KB family members and their activatability through CD40 or BCR were analyzed via WB. Upon activation of the NF-KB signaling pathway, the transcription factors belonging to the NF-KB family translocate into the nucleus, which can be monitored by extracting nuclear and cytosolic proteins separately after giving the stimulus to the cell. Thus, the model cell line for Burkitt's lymphoma in the present study, BL2, as well as a model cell line for DLBCLs, OCI Ly1, were compared with regard to the ability to translocate p65, c-Rel, or RelB to the nucleus 1 hour after activation with either SCD40L or $\alpha$-IgM antibody (Figure 3-15 A \& B). Yet, a more direct observation of NF-kB activation via nuclear translocation of the respective transcription factor was achieved in comparison to the indirect proof of NF-KB activation by IKB $\alpha$-degradation.

Unexpectedly, in both cell lines, BL as well as DLBCL, a nuclear translocation of the NF-KB family members p65, c-Rel and RelB was mainly detectable after CD40 activation, but not after $\alpha$-IgM stimulation. In the BL2 cell line, a remarkably strong nuclear signal for p65 was detectable in the unstimulated cells as well, thus favoring a completely new and contradicting theorem of constitutive active NF-KB signaling in the BL2 cell line. Indeed, when pre-treating BL2 cells with an IKK2-inhibitor, both the basal and the CD40 activated nuclear p65 signal was diminished (Figure 3-15 C). Unexpectedly, no BCR induced nuclear translocation of any investigated NF-KB family member was detectable in the DLBCL cell line OCI Ly1 (Figure 3-15 F). Instead, the BL cell line BL2 exhibits a faint NF-KB activation signal 60 min after $\alpha$-IgM stimulation, which can be inhibited through either IKK2 or Tak1 inhibition (Figure 3-15 E). However, a low grade of NF-KB activation can be guessed in terms of nuclear translocation of p65, but not the other NF-KB family members c-Rel or RelB. 
A

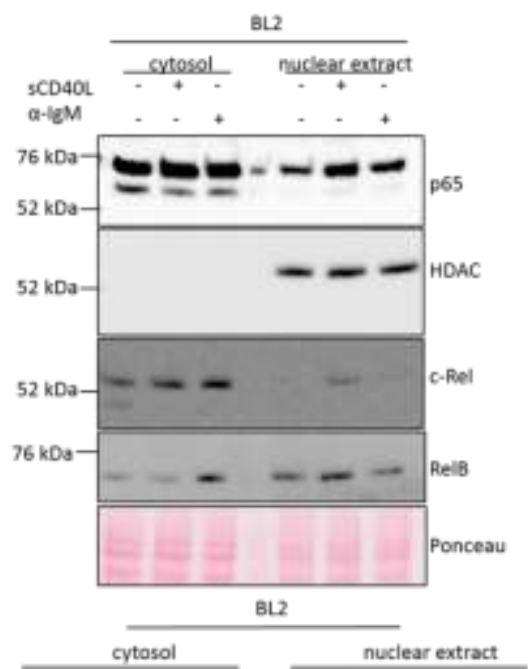

C
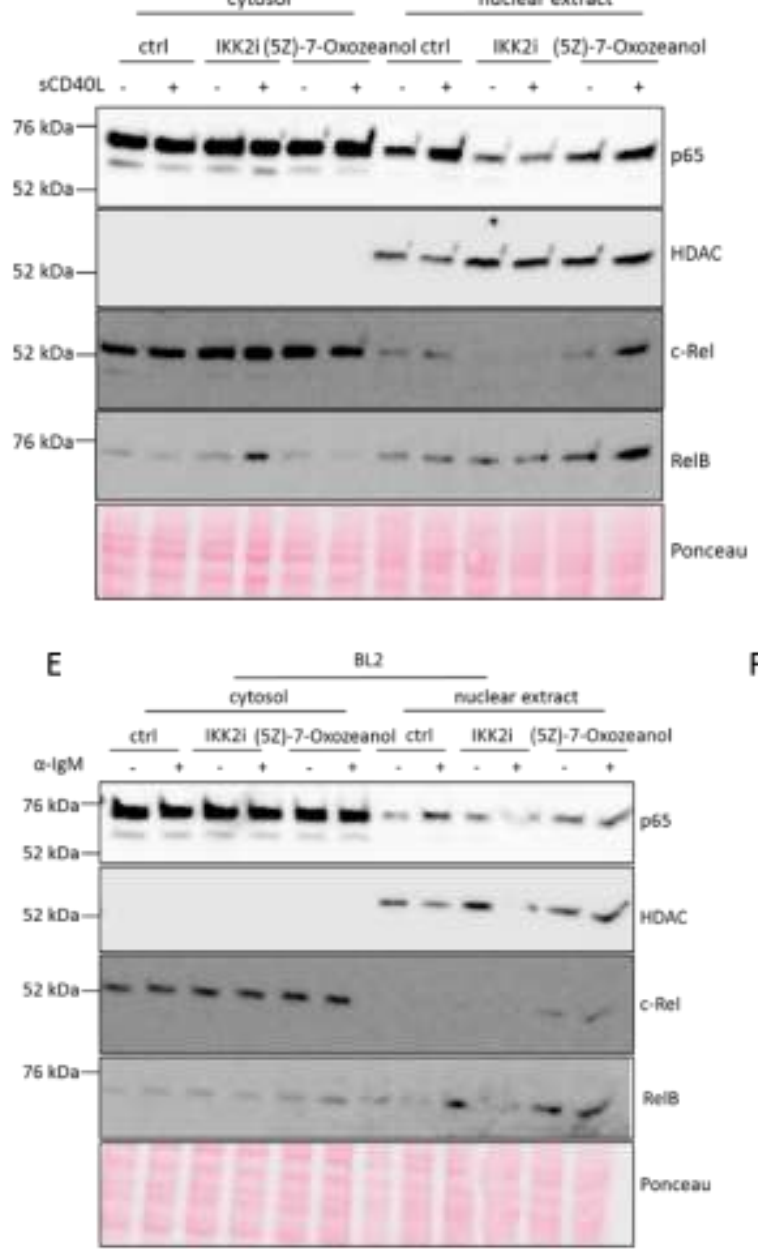

B

D

F
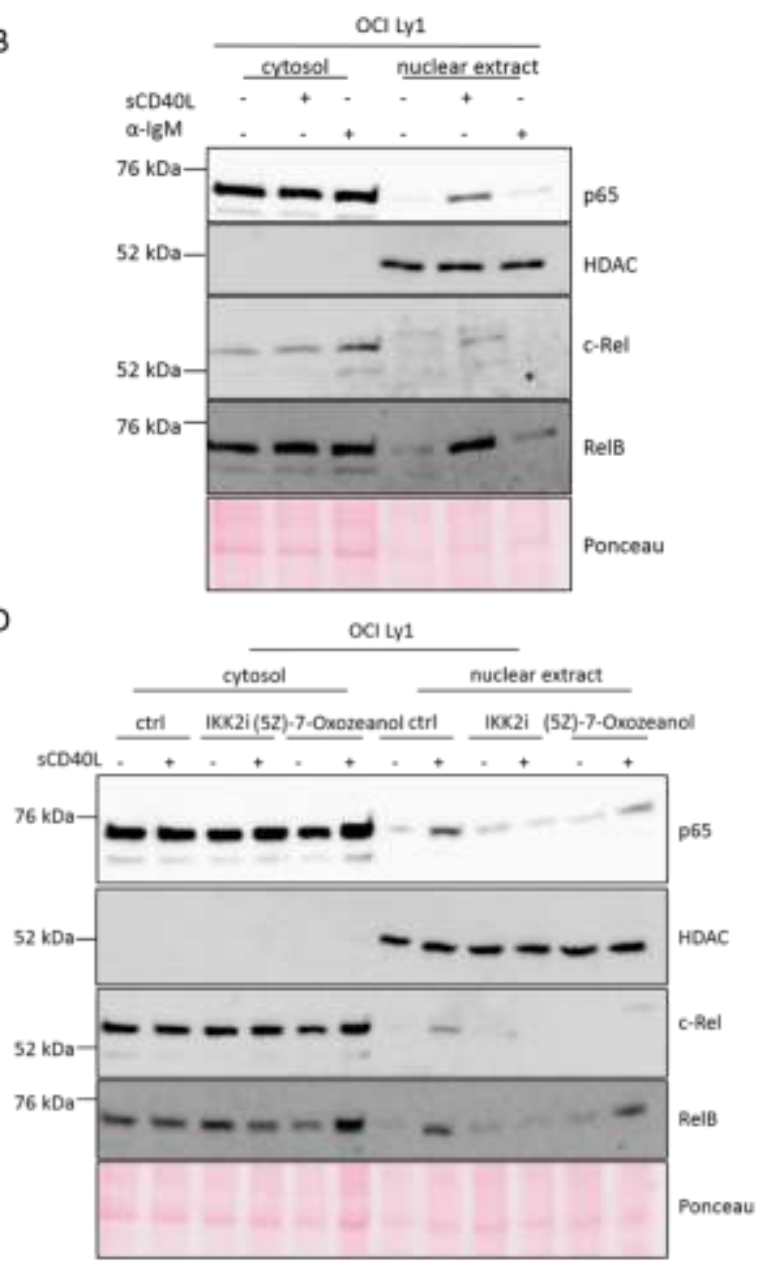

oc: Ly1

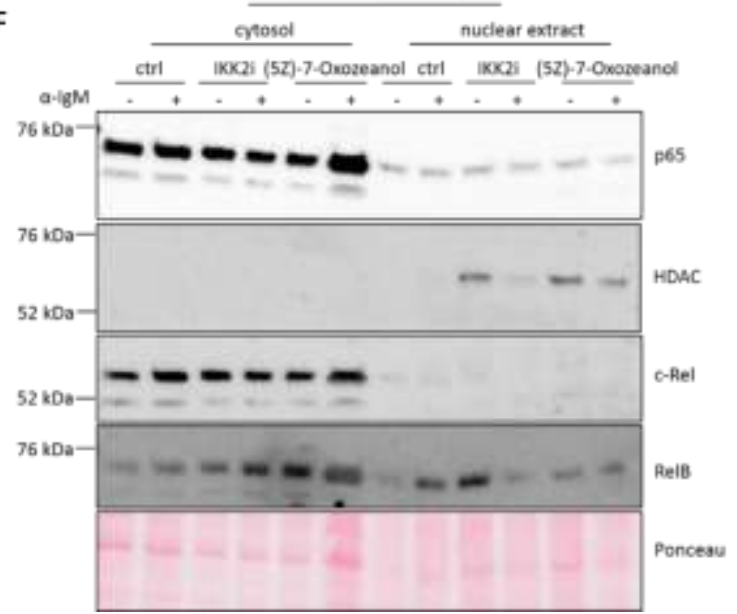

Figure 3-15 Differences in NF-kB activatability in Burkitt's lymphoma and Diffuse large B cell lymphoma. Nuclear translocation of NF-kB family member was monitored using specific antibodies against p65, c-Rel and Rel $b$. The nuclear extract was verified using a specific antibody against the nuclear marker HDAC1. To compare the differences between Burkitt' lymphoma and diffuse large $B$ cell lymphoma, experiments were carried out in BL2 cells (BL) (A, C, E) and OCI Ly1 (DLBCL) (B, D, F). A + B show the general activatability of nuclear NF-kB translocation through CD40 stimulation and BCR crosslink with antibodies specific for NF-KB family members p65, c-Rel and RelB. C + D depict WB analyses of BL2 and OCI Ly1 cells upon NF-kB inhibition (IKK2i) and Tak1 inhibition ((5Z)-7-Oxozeanol) and 60 min CD40 stimulation. E + F show the same analyses as C + D upon stimulation with $\alpha-\operatorname{lgM~} F(a b)_{2}$ fragment. $10 \mathrm{ng}$ of protein were loaded for each fraction. The purity of the nuclear extracts was detected using a specific antibody against Histone Deacetylase 1 (HDAC). 
To conclude, the nuclear translocation of p65 upon $\alpha$-IgM stimulation demonstrates that NF-kB signaling seems to be slightly activated after BCR activation in the BL cell line BL2. This stands in contrast to the observed stabilization of IKB $30 \mathrm{~min}$ and $60 \mathrm{~min}$ after BCR stimulation. However, CD40 mediated activation of NF-KB signaling is more pronounced when compared to BCR mediated NF-KB activation. Additionally, the BL2 cell line harbors high basal levels of nuclear p65 thus suggesting a basal NF-KB activity. Moreover, we observed for the first time, that BCR activation could not induce nuclear translocation of NF-KB family members in a DLBCL cell line. However, the molecular mechanisms of this phenomenon remain to be elucidated. 


\section{Discussion}

We postulated that the differences between molecular BL and DLBCL are due to specific immune response associated signals mediated by the B cell microenvironment. Using in vitro stimulation of human transformed germinal center B cells as a model system to investigate pathway activation in aNHL, we could already demonstrate that both B cell receptor activation as well as CD40 activation lead to gene expression changes, which can be used to discriminate individual DLBCLs (Schrader et al., 2012b). However, these data are restricted to a specific time after giving the stimulus and may hide additional information about potential oncogenic pathway activities and their regulation. Thus, the present study aimed to provide gene expression data, which allow to investigate the relevant signaling pathways and their consequences in more detail. Therefore, this study used (a) timeresolved analysis of gene expression changes upon oncogenic pathway activation and (b) a combination of in vitro stimulation and kinase inhibition to provide data allowing the prediction of regulatory feedback loops and delineate respective pathway interactions on the whole genome gene expression level.

\subsection{Analyzing the temporal pattern of gene expression changes}

It was shown in this study that $\alpha$-IgM as well as CD40 stimulation activate several signaling pathways in the BL cell line BL2. This includes overlapping signaling as the activation of p38 MAPK pathway through CD40 and $\alpha$-IgM as well as distinct pathways, activated specifically through one of both stimuli. Thus, CD40 activation induces a strong and rapid activation of NF-KB signaling, whereas the $\mathrm{BCR}$ mediated NF-KB activation seems to be less pronounced and delayed in comparison to CD40 mediated activation (compare Figure 3-1 C \& E). Activation of ERK/MAPK and PI3K/AKT pathway are unique to BCR mediated signaling and not triggered through CD40 induced signaling activation.

To our knowledge this study provides for the first time a global time-resolved investigation on the whole genome level of gene expression changes upon signaling pathway activation through CD40 or $\mathrm{BCR}$ crosslink on B lymphocytes. Both, BCR activation as well as CD40 activation induced a unique gene expression profile as revealed by microarray analyses. As shown before in another study, $\alpha$-IgM stimulation of transformed germinal center B cells led to differential expression of significantly more genes than CD40 activation (Schrader et al., 2012b).

It was shown in this study that especially genes which are differentially expressed upon $\alpha$-IgM stimulation shift the gene expression profile of $\mathrm{mBL}$ towards a gene expression profile of DLBCL (Figure 3-2). It becomes clear, that sustained activated signaling via the B cell receptor but not activation of CD40 mediated signaling leads to changes in gene expression which reduce the $\mathrm{mBL}$ index. This observation is supported by recent findings, which demonstrate chronic active BCR signaling in a subgroup of DLBCL, the ABC-like DLBCL (Davis et al., 2010; Kloo et al., 2011; Ngo et al., 
2006), thus defining the molecular profile of $A B C D L B C L$ in comparison to other DLBCL subtypes (Alizadeh et al., 2000). In addition, our group could very recently demonstrate that the coherent expression of $\alpha$-IgM affected genes characterizes individual NHL (Schrader et al., 2012b). In line with recent advances in the understanding of lymphoma pathogenesis, one can conclude that B cell receptor signaling has emerged as a central oncogenic pathway in lymphoma genesis and progression. The cascade of effectors downstream of the BCR includes many kinases diversifying the signal intensity, amplitude and outcome. Thus, it is not surprising that lymphomas adopt and spoil this signaling pathway, rendering it to an essential tool, which bears the potential of targeted therapy for patients with aNHL.

Due to the high-dimensionality and complexity of the generated data, statistical and bioinformatical analyses turned out to be challenging. In the present study, two different methods were used to analyze the temporal pattern of gene expression changes, thereby aiming at the disclosure of regulatory circuits, positively and negatively influencing signaling routes in their top-todown signaling cascade. Even though using distinct mathematical models, both analyses are based on the integration of gene expression changes with similar time course to clusters of genes. It is not surprising that the computed clusters of genes are not identical to each other, since different mathematical approaches may result in different major findings, an observation which was demonstrated by others as well (de Matos Simoes et al., 2012). Apparently, in both models, BCR as well as CD40 activation induce a variety of different gene expression changes, including immediateearly activated and repressed genes as well as genes being changed in their expression profile after long-term stimulation or genes, displaying more-phasic time courses.

The first approach presented in this study demonstrates a causality network of $\alpha$-IgM mediated gene expression correlations (Figure 3-3) and their conditional dependency, inferring an influence between connected clusters of genes (Chiquet et al., 2009). The network nicely demonstrates the complexity and connectivity of signaling pathways, which are induced upon crosslinking the BCR. A biological interpretation of this comprehensive network still remains to be fully elucidated. A first attempt to assign GO-terms and search for significant enrichment of specific biological processes identified the hub clusters of the causality network to be enriched for genes encoding kinases, Gproteins and related proteins as well as genes encoding for transcription factors thus playing crucial roles in cell signaling and cell proliferation processes. However, further analyses revealed that several clusters resemble each other not only in gene expression course but also in the biological processes the genes are encoded for. Furthermore, most clusters are composed of genes, which cannot be assembled to one specific function or process and whose composition remains difficult to interpret. 
Tremendous advances have been made in the development of new mathematical and bioinformatical methods to construct gene regulatory networks (Friedman, 2004; Lee and Tzou, 2009; Markowetz and Spang, 2007). These methods often combine gene expression data with for example protein-DNA interaction data to recover the underlying networks. However, most methods focus on reconstructing networks from static data and do not contain any temporal information. Only very recent investigations dealed with time series expression data and provided some interesting new methods to engineer gene regulatory networks (Cooke et al., 2011; Darkins et al., 2013; Gonçalves et al., 2012; Haye et al., 2012; Nascimento et al., 2012; Schulz et al., 2012; Su et al., 2013; Titsias et al., 2012; Yeung et al., 2011; Zou and Conzen, 2005). Thus, the conflation of our time course specific gene expression data set with other sources of prior knowledge may be useful to fathom the biological meaning of such a causality network. In order to shed some light on the complexity of the gene expression changes and their causal correlation induced through $\alpha$-lgM stimulation, ongoing analyses are supposed to integrate genome-wide chromatin immunoprecipitation assays in our cell perturbation assays. Therewith, we aim to use such a causality network for identification of, for example, transcription factor targets.

The mathematically further developed and refined method of testing for stationarity applied by J. Läuter and colleagues was used to build sets of genes which display distinct expression changes over time. Hereby, the clustering of genes is strictly mathematically justified and the biological interpretation relies on the assumption that genes, which exhibit a uniform process are also regulated through the same elicitor or display a similar biological function. Combining this method of time course analysis with Gene Ontology enrichment allowed the discrete characterization of stimuliinduced gene expression changes over time on a global level. One can conclude from the present study that activation of target genes affecting proliferation, differentiation and paracrine signaling occurs 2 hours after CD40L activation and thus, CD40 mediated signaling happens very rapidly on the proteome-level, leading to a fast feedback loop mediated by gene expression changes. In contrast, $\mathrm{BCR}$ mediated signals are also translated rapidly into gene expression changes but seem to induce a broader variety of signals. This is justified by the higher number of differentially expressed genes when compared to CD40 induced gene expression changes as well as longer lasting changes in gene expression. Taken together with the observation of the BCR mediated shift of originally $\mathrm{mBL}$-indexbearing cells towards a gene expression pattern of non-mBL (chapter 3.1.1), the observations made in the present study underpin the particular importance of B cell receptor signaling in lymphoid malignancies. This is in line with recent assessments and the related investigations in the field of targeting the B cell receptor clinically (reviewed in Young and Staudt, 2013). 
As described in chapter 3.1.3, time series analysis of $\alpha$-IgM induced gene expression changes demonstrated the temporal expression pattern of many well-known and pivotal players in both normal and pathological BCR signaling. One example is CCR7, a chemokine receptor shown to be an essential regulator of leukemia cell infiltration into the central nervous system (Buonamici et al., 2009). Its high expression in T-cell leukemias was associated with tumor cell migration and invasion in vitro and in vivo (Yang et al., 2011). Further, CCR7 was recently described for regulating homing of lymphoma cells to $\mathrm{T}$ cell regions in lymph nodes, thus promoting lymphoma cell survival through facilitating the cross-talk to other microenvironmental non-tumor cells (Rehm et al., 2011). In addition, Rehm and colleagues suggested that T-cell zone location of their mouse-model-derived lymphoma cells awards resistance to spontaneous apoptosis through the proximity to fibroblastic reticular cells (FRCs), which provide the hedgehog protein Indian hedgehog, as shown by reduced viability of lymphoma cells upon use of the Hh signaling inhibitor Cyclopamine. This very interesting observation taken together with the simultaneous BCR mediated down regulation of the Hh-signaling receptor PTCH1 as well as LEF1 and NOTCH1 (compare Figure 3-5 and Figure 3-6), both pivotal players in developmental biology as well as being associated with several types of lymphoid malignancies (Cheng et al., 2001; Pui et al., 1999; Radtke et al., 2004; Reya et al., 2000; Walther et al., 2013), strengthens the importance of paracrine and autocrine signaling in the tumor microenvironment and prompted us to a more profound analysis of the role of hedgehog signaling in lymphoma. 


\subsection{A non-canonical role of Hedgehog signaling in lymphoma}

The statistical analyses of BCR mediated gene expression changes identified the BCR induced corepression of LEF1, NOTCH1 and PTCH1. Lef1, a transcription factor in the Wnt signaling pathway, has been shown to regulate normal B lymphocyte proliferation (Reya et al., 2000). Further, LEF1 is one of the signature genes defining the $\mathrm{mBL}$ index (Hummel et al., 2006) and very recently our group could demonstrate an aberrant LEF1 expression as well as its transcriptional activity in BLs (Walther et al., 2013). Notch1, the transmembrane receptor for the Notch signaling network plays not only a role in lymphocyte development, proliferation and differentiation (Anderson and Longnecker, 2009; Cheng et al., 2001; Pui et al., 1999; Radtke et al., 2004) but has also been implicated in a variety of hematological malignancies including chronic lymphatic leukemia (CLL) (Okuyama et al., 2012; Willander et al., 2013), acute lymphatic leukemia (ALL) (Schwanbeck and Just, 2011; Tosello and Ferrando, 2013) as well as DLBCL (Lohr et al., 2012). Further on, both genes are connected to c-MYC activation (Rakowski et al., 2013; Yochum et al., 2008), which in turn displayed a similar time course of mRNA decrease after BCR crosslink. However, the connection of Ptch1 and related hedgehog signaling and c-Myc in lymphoma signaling remained elusive so far. Studies in Hh-induced medulloblastoma formation suggested that c-Myc activates GLI1 expression in vitro and thus promotes tumorigenesis (Rao et al.; Zwerner et al., 2008). Evidence for the role of Hh in lymphoma is limited but rapidly developing. Both, healthy germinal center B cells as well as myc-driven lymphoma cells have been found to be protected from apoptosis by Hh ligands secreted by stroma cells (Dierks et al., 2007; Sacedón et al., 2005). Further, expression of Hh signaling proteins and their contribution to survival was described for some DLBCL cell lines (Singh et al., 2010). However, the relevance of Hh signaling in BL cells was not determined. In the present study, the analyzed BL cell lines differed strongly in their basal expression level of Hh signaling components. Hh ligands as $S H H$ and $I H H$ were not or only barely expressed by BL cell lines. Further on, activation of the Hh signaling pathway could not be achieved through stimulation of the cells with Shh-enriched medium, thus suggesting that BL cells are not responsive to Hedgehog pathway activation. These results are supported by a very recent study, confirming that Burkitt's lymphoma cells do not seem to be Hedgehog responsive (Yoon et al., 2013). Instead, this group shows that c-Myc directly regulates GLI1 expression more significantly than the canonical Hh signaling pathway. This finding circumstantiates the here presented data, which demonstrate no relevance of SMO inhibition on BL cells. Additionally, the BCR induced c-Myc repression occurs slightly earlier when compared to the time course of PTCH1. These findings suggest a c-Myc driven non-canonical regulation of $\mathrm{Hh}$ signaling in $\mathrm{BL}$ and implies new therapeutic approaches, which include the direct targeting of Gli1 or GLI1 target genes.

This study could not reveal generally higher expression levels of Hh signaling components in the analyzed DLBCL cell lines, although several studies from one group around F. Vega suggest active $\mathrm{Hh}$ 
signaling in $A B C D L B C L$, including expression and secretion of $\mathrm{Hh}$ ligands as well as responsiveness to Hh-inhibition (Ok et al., 2012; Ramirez et al., 2012; Singh et al., 2009, 2010, 2011). These findings are somewhat contradictory, even though they might be explainable by the diversity of the cell lines used for the investigations in the present and the recently published studies.

However, the observation of $\mathrm{BCR}$ mediated down regulation of $P T C H 1$ expression raised the initial hypothesis that Burkitt's lymphoma harbor active Hh signaling. As shown in Figure 3-6, the computed time course of PTCH1 was verified by qRT-PCR and revealed an activation of PTCH1 gene expression after $\alpha$-IgM stimulation before its expression was down regulated. Thus, the observed reduction of PTCH1 mRNA levels after 3 to 4 hours of BCR activation might contribute to a regulatory feedback loop, preventing an exaggeratory Hh response. However, activation of BCR signaling in the BL cell line BL2 led to reduced levels of PTCH1 gene expression but no changes in expression levels of GLI1 (Figure 3-7). Taken together with the contrasting results in other BL cell lines, we concluded that canonical Hh signaling is not active in BLs. Instead, the observed elevated GLI1 expression levels upon $B C R$ activation in most cell lines favor the hypothesis of active Hh signaling in DLBCLs. This especially holds true when taking into consideration that $B C R$ activation induces a transition of the $\mathrm{mBL}$ index from $\mathrm{mBL}$ towards DLBCL.

Activation of Hh signaling in cancer has been attributed to aberrant activation of several signaling pathways resulting in the integration of multiple oncogenic signaling inputs. Interplay of TGF- $\beta$, NF$K B$, Notch and the ERK/MAPK pathway is supported by multiple lines of evidence (Chang et al., 2010; Madhala-Levy et al., 2012; Mangelberger et al., 2012; Qu et al., 2013; Schnidar et al., 2009) Furthermore, a synergistic effect between $\mathrm{Hh}$ and PI3K signaling was suggested for several cancer entities including ALK (anaplastic lymphoma kinase)-positive anaplastic large cell lymphoma cells (Ju et al., 2009; Mizuarai et al., 2009; Singh et al., 2009; Wei and Xu, 2011). Very recently, a study defining causative factors for activation of Hh signaling in diffuse large B cell lymphoma supported this link, by demonstrating reduced Gli1 protein levels after PI3K inhibition in DLBCL cell lines (Ramirez et al., 2012). However, they could not reveal the precise mechanism by which PI3K regulates Hh signaling. Furthermore, in this study Gli1 protein expression was inhibited by very high concentrations of the PI3K inhibitor Ly294002 in GCB DLBCL cell lines, whereas in the ABC DLBCL cell line OCI Ly3, which exhibits chronic active BCR signaling (Davis et al., 2010), lower concentrations of Ly294002 were sufficient to effectively abrogate Gli1 protein expression (Ramirez et al., 2012). Similarly, long-lasting inhibition of PI3K through Ly294002 also increased PTCH1 and additionally cMYC expression levels in unstimulated BL2 cells (Schrader et al., 2012b and unpublished own data). This not only supports the already suggested link between PI3K and Hh signaling but additionally connects c-Myc activity to PI3K and Hh activity in lymphoma biology. Indeed, a synergistic mode of 
action of c-Myc and PI3K has been recently described for lymphoma pathogenesis (Sander et al., 2012). Further on, a basal activity of PI3K and tonic BCR signaling was demonstrated in Burkitt's lymphoma (Schmitz et al., 2012).

Nonetheless, the present study revealed a strong heterogeneity between different $\mathrm{BL}$ cell lines regarding their $B C R$ mediated $\mathrm{Hh}$ signaling activation. Furthermore, cell lines where the $B C R$ activation led to PTCH1 expression level reduction not necessarily displayed activation of $\mathrm{Hh}$ signaling as monitored by GLI1 expression. These findings support a non-canoncial activation of $\mathrm{Hh}$ signaling in lymphoma cells as recently proposed by others (Yoon et al., 2013). Similarly, the cell lines tested in the present study did not show any responsiveness to canonical Hh signaling activation through stimulation by Shh. In summary, the here presented investigations could not reveal a clear BCR induced activation or inactivation of canonical Hh-signaling in lymphoma cells. Moreover, Burkitt's lymphoma cells as well as the one DLBCL cell line investigated here do not seem to be Hedgehog responsive. Above all, the present study could not exclusively determine the same regulatory factor behind the simultaneous decrease in expression levels of $c-M Y C$, PTCH1 and LEF1, although the regulation of all three genes is clearly dependent on the PI3K-PLCY-PKC pathway. Taken together, the here presented results favor a non-canonical role of Hh signaling in lymphoma. 


\subsection{Analyzing pathway intersections}

Our group was recently able to mimic oncogenic pathway activities in aNHL by investigating the major patterns of gene expression changes in response to different immune response-associated stimuli in vitro (Schrader et al., 2012b). We were also able to identify individual aNHL profiles reflecting those patterns and we concluded that future targeted therapies have to intervene into specific signaling modules to reverse pathway deregulations. Therefore it is of crucial relevance to understand the constellation of oncogenic activities in the context of their interplay and the cross talk of signaling pathways being involved in the pathogenesis of lymphomas. Thus, the present study aimed to use a combination of in vitro stimulation with specific kinase inhibitors in order to delineate respective pathway interactions. The genome-wide expression profile of BL2 cells was investigated in several conditions, depicting oncogenic signaling activation and its abrogation at specified intersections. Thus, a large amount of comprehensive data was generated, which is still subject to work in progress in order to establish suitable methods for analyzing the complexity, the connectivity and the biological relevance of gene expression changes.

\section{Tak1 as major modulator in CD40 and BCR mediated signaling}

A recent computational approach to infer signaling pathway structures from perturbation experiments and subsequent gene expression profiling, Nested Effects Modeling (NEM) (Markowetz et al., 2005, 2007), was used to compare the role of Tak1 in CD40 and BCR mediated signaling. In CD40 activated signaling in B cells, Tak1 has been described to act as pivotal signal regulator upstream of p38, JNK and NF-KB activation (Sato et al., 2005; Takaesu et al., 2003; Yamashita et al., 2008). BCR signaling also activates those signaling pathways, but the contribution of Tak1 is controversially debated. Sato and colleagues demonstrated murine Tak1-deficient B cells to be able to still activate NF-KB signaling, but not the kinase JNk in response to BCR stimulation (Sato et al., 2005), whereas a later study demonstrated that B cell-specific TAK1-deficiency disrupted BCR induced NF-KB activation (Schuman et al., 2009).

However, the NEMs derived from the gene expression data in the present study proposed an unexpected dispensable role for Tak1 in CD40 and BCR mediated signaling BL cells (Figure 3-12). Yet, subsequently conducted biochemical investigations provided strong evidence that the primarily proposed nested effects models are not sufficient to describe the role of Tak1 in CD40 and BCR mediated signaling. Comparative analysis of the two independent inhibitors of Tak1 activity, (5Z)-7Oxozeanol and AZ-TAK1, as well as Tak1-directed siRNA revealed the indispensability of Tak1 for mediation of p38 and NF-KB activation in CD40 mediated signaling. In BCR activated signaling, the Tak1 mediated activation of Erk, besides p38 and NF-KB activation was demonstrated. Thus, former results from other studies can be transferred to BL cells. Tak1 is a pivotal modulator of p38, JNK and 
NF-KB signaling in CD40 as well as BCR activated pathways. This includes a so far not recognized regulatory role of Tak1 in Erk1/2-signaling. The present results suggest, that inhibitors targeting Tak1 activity might be suitable treatment options for lymphoma subtypes, depending on NF-KB, JNK, p38 or Erk1/2 signaling activity. This is supported by a very recent report, which was published during the work on the current study and revealed a pivotal role for Tak1 in regulating cell survival of mantle cell lymphoma cells, a subtype of aNHL exhibiting active NF-kB signaling (Buglio et al., 2012).

The here conducted analysis highlights the limitations of NEMs to handle such complex highdimensional data in order to correctly return upstream/downstream relations of cellular signaling cascades and demonstrates the need for adaptation of the existing method. The observed differences in signal intensity may lead to the construction of dissatisfactory graphs. The gene expression changes induced through $\mathrm{BCR}$ stimulation were much stronger than those induced through CD40 activation. Similar observations were made for use of the different inhibitors. Thus, less genes are significantly strong regulated upon p38 inhibition than upon Erk1/2 inhibition. Since a very stringent cut-off ( $p$-value) for calculation of the NEMs is used, it is possible that such systemrelevant effects cannot be spotted and lead to the omission of potential p38-exerted effects. Therefore, a new method of discretization has now been established to integrate the different signal intensities in future modeling processes (personal communication from M. Pirkl, Regensburg). Another possible cause for the limitations in the NEMs is lying within the off-target effects of the inhibitors. The difficulty in modeling signaling cascades with the help of intervention studies is concentrated on the possibility to smartly differentiate off-target effects from real pathway signaling. Therefore, we are now primarily interested in establishing new methods for learning regulatory networks from compendia of the perturbation expression data and combining this data with data sources that provide prior knowledge to the network structure. One source of such prior information is our collection of manually curated and experimentally validated data, obtained from Western Blot analyses, qRT-PCRs or reporter-gene assays. Additionally, literature-based prior information will also be included in the generation of a new model. Thus, adjustement of the existing statistical algorithms is in preparation in order to map a biologically comprehensive and perspicuous regulatory network of the here analyzed perturbations.

\section{$B C R$ dependent NF-KB signaling in BLs}

Very recent studies demonstrated a so-called 'tonic' BCR signaling of Burkitt's lymphoma cell lines, as shown through killing of BL cells by CD79A- or SYK-knock down (Schmitz et al., 2012). Consistent with these findings, conditional activation of PI3K along with Myc in mouse germinal center B cells leads to lymphoma genesis of strong BL phenotype (Sander et al., 2012). This 'tonic' BCR signaling in Burkitt's lymphoma activates the PI3K pathway, but is interestingly not dependent on the NF-KB 
signaling-mediators CARD11 or BTK. This demonstrates the qualitative difference to 'chronic active' BCR signaling in ABC DLBCL (Davis et al., 2010; Schmitz et al., 2012).

In contrast to other lymphoma entities as DLBCLs or mantle cell lymphoma, Burkitt's lymphoma are generally reported to be characterized through low expression levels of NF-kB activation modules (Dave et al., 2006; Hummel et al., 2006). Moreover, constitutive activation of the normally prosurvival factor NF-KB increased cell death in several BL cell lines (Klapproth et al., 2009).

Typically, antigen-mediated activation of the B cell receptor leads to activation of NF-kB signaling in $B$ cells via activation of PKC $\beta$ and subsequent signal adaptors like CARMA1, Bcl10 and MALT1 (Egawa et al., 2003; Li and Verma, 2002; Ruefli-Brasse et al., 2003; Ruland and Mak, 2003). Contrarily, the current study demonstrates a reduced activatability of NF-KB signaling through BCR activation when compared to CD40 mediated signaling in the BL cell lines BL2 and Ramos (Figure 3-14). Moreover, the GCB DLBCL cell line OCI Ly1 did not exhibit a BCR inducible NF-KB activation either. A role for 'tonic' BCR signaling has been postulated for GCB DLBCLs as well (Chen et al., 2008), although genetic knockdown of BCR components did not affect the survival of various GCB DLBCL cell lines (Davis et al., 2010). Therefore, it seems possible that some so far unknown mechanisms of 'tonic' BCR signaling prevent a profound activatability of NF-KB signaling. Another possibility, which has to be elucidated, is a delayed activation of NF-KB signaling upon antigen-mediated BCR activation on the background of 'tonic' BCR activity, since BL2 cells exhibit low levels of nuclear translocation of NF-KB transcription factors after $60 \mathrm{~min}$ of $\alpha$-IgM stimulation. Furthermore, time course analysis of $\alpha$-IgM stimulated BL2 cells revealed a NF-KB activation after 60-90 min of BCR crosslink, as visualized by faint degradation of IKB $\alpha$ (Figure 3-1). However, when comparing the nuclear levels of NF-KB family members, remarkably high levels of nuclear p65 were detected in unstimulated BL2 cells, which were abolished through IKK2-inhibition. Thus, one can conclude that the BL2 cell line exhibits constitutive active NF-KB signaling. This completely new and former findings-contradicting hypothesis is sustained through preliminary data, which show reduced proliferation of BL2 cells upon IKK2-inhibition (data not shown). Further studies in several other BL cell lines should elucidate if the activation of NF-KB, including the translocation of its transcription factor molecules into the nucleus, is abrogated at a later time in BL signaling or if this phenomenon exclusively holds true for the BL2 cell line. As also shown in Figure 3-14, the CD40 mediated NF-KB activation is in Ramos cells not ablated through Tak1-inhibition as it is in BL2 cells. In spite of the confirmed pivotal role of Tak1 as major modulator of antigen and CD40 induced signaling pathways in $\mathrm{BL}$ cells, the direct comparison of the two BL cell lines Ramos and BL2 with regard to Tak1 mediated signaling exposed once again the heterogeneity of the available BL cell lines and the probably particular role of the BL2 cell line as model cell line for Burkitt's lymphoma (not published data from the group of D. Kube). 


\section{Conclusion}

The analyses of the time course of global gene expression changes of BCR or CD40 stimulated human transformed germinal center $B$ cells enabled us to describe distinct gene groups affected in a comparable way, being either activated or suppressed. Therewith, new regulatory circuits and perhaps feedback loops can be postulated. A co-repression of LEF1, PTCH1 and NOTCH1 mRNA expression through BCR activation was identified, which is slightly delayed when compared to the BCR induced c-Myc repression. This could represent one mechanistic explanation for the observed shift of the $\mathrm{mBL}$ signature of the BL2 lymphoma cell line towards the non-mBL gene expression profile. However, this needs to be proven by additional investigations, as for example testing different cell lines with differing c-Myc expression levels. The additional treatment with other factors of the DLBCL microenvironment or a combination of stimuli could be helpful to avoid the observed reversion of BCR mediated gene expression by so far unknown feedback loops. Furthermore, causal relationships within oncogenic pathways for BCR activated human transformed germinal center $B$ cells were proposed but need further prior knowledge integration before experimental validation.

The current study provide evidence that, although PTCH1 mRNA expression levels are changed upon BCR activation, active Hh signaling does not seem to be important for Burkitt's lymphoma. Instead, we suggest a non-canonical regulation of Hh signaling in cross talk with aberrant c-Myc expression. Furthermore, the biochemical validation of a computationally compiled model of Tak1signaling revealed a context-dependent role of Tak1 in the modification of signaling transduction. Thus, a direct contribution of Tak1 to the BCR induced phosphorylation of Erk1/2 was demonstrated for the first time. In addition, the present study provides evidence for a constitutive active NF-KB signaling in the BL2 cell line and suggests reduced NF-KB activatability in tonic BCR signaling.

These global gene expression analyses offer comprehensive data collections, which can be used in lymphoma research but also modeling of oncogenic pathways in general. Therefore, herein created data provide a sophisticated basis to develop new bioinformatical and statistical methods fostering a better understanding of signaling pathways and their regulatory feedback mechanisms. However, the present study also elicits vigorously the balance between prediction of oncogenic pathway activites and prior knowledge integration to put forward corresponding new hypotheses for experimental validation. In addition, we conclude that isolated global gene expression profiling needs to be complemented by additional pathway analyses. As the cell fate is not only determined by gene expression changes but also by posttranslational protein modifications as phosphorylations, ubiquitinations, degradations and others, the integration with data sets obtained by other -omics may be helpful for future characterizations of oncogenic pathways in lymphoma pathogenesis and progression. 


\section{Bibliography}

Ahn, T., Yim, S.K., Choi, H.I., and Yun, C.H. (2001). Polyacrylamide gel electrophoresis without a stacking gel: use of amino acids as electrolytes. Analytical Biochemistry 291, 300-303.

Akiba, H., Oshima, H., Takeda, K., Atsuta, M., Nakano, H., Nakajima, A., Nohara, C., Yagita, H., and Okumura, K. (1999). CD28-independent costimulation of T cells by OX40 ligand and CD70 on activated B cells. Journal of Immunology (Baltimore, Md. : 1950) 162, 7058-7066.

Alizadeh, A.A., Eisen, M.B., Davis, R.E., Ma, C., Lossos, I.S., Rosenwald, A., Boldrick, J.C., Sabet, H., Tran, T., Yu, X., et al. (2000). Distinct types of diffuse large B-cell lymphoma identified by gene expression profiling. Nature 403, 503-511.

Amini, R.-M., Berglund, M., Rosenquist, R., Von Heideman, A., Lagercrantz, S., Thunberg, U., Bergh, J., Sundström, C., Glimelius, B., and Enblad, G. (2002). A novel B-cell line (U-2932) established from a patient with diffuse large B-cell lymphoma following Hodgkin lymphoma. Leukemia \& Lymphoma 43, 2179-2189.

Anderson, L.J., and Longnecker, R. (2009). Epstein-Barr virus latent membrane protein 2A exploits Notch1 to alter B-cell identity in vivo. Blood 113, 108-116.

Antony, P., Petro, J.B., Carlesso, G., Shinners, N.P., Lowe, J., and Khan, W.N. (2004). B-cell antigen receptor activates transcription factors NFAT (nuclear factor of activated T-cells) and NF-kappaB (nuclear factor kappaB) via a mechanism that involves diacylglycerol. Biochemical Society Transactions 32, 113-115.

Arcipowski, K.M., and Bishop, G. a (2012). Roles of the kinase TAK1 in TRAF6-dependent signaling by CD40 and its oncogenic viral mimic, LMP1. PloS One 7, e42478.

Aszterbaum, M., Epstein, J., Oro, A., Douglas, V., LeBoit, P.E., Scott, M.P., and Epstein, E.H. (1999). Ultraviolet and ionizing radiation enhance the growth of BCCs and trichoblastomas in patched heterozygous knockout mice. Nature Medicine 5, 1285-1291.

Aversa, G., Punnonen, J., and de Vries, J.E. (1993). The 26-kD transmembrane form of tumor necrosis factor alpha on activated CD4+ T cell clones provides a costimulatory signal for human B cell activation. The Journal of Experimental Medicine 177, 1575-1585.

Baldwin, A.S. (2001). Control of oncogenesis and cancer therapy resistance by the transcription factor NF-kappaB. The Journal of Clinical Investigation 107, 241-246.

Beckwith, M., Longo, D.L., O'Connell, C.D., Moratz, C.M., and Urba, W.J. (1990). Phorbol esterinduced, cell-cycle-specific, growth inhibition of human B-lymphoma cell lines. Journal of the National Cancer Institute 82, 501-509.

Behrens, T.W., Jagadeesh, J., Scherle, P., Kearns, G., Yewdell, J., and Staudt, L.M. (1994). Jaw1, A lymphoid-restricted membrane protein localized to the endoplasmic reticulum. Journal of Immunology (Baltimore, Md. : 1950) 153, 682-690.

Ben-Bassat, H., Goldblum, N., Mitrani, S., Goldblum, T., Yoffey, J.M., Cohen, M.M., Bentwich, Z., Ramot, B., Klein, E., and Klein, G. (1977). Establishment in continuous culture of a new type of 
lymphocyte from a "Burkitt like" malignant lymphoma (line D.G.-75). International Journal of Cancer. Journal International Du Cancer 19, 27-33.

Bennett, B.L., Sasaki, D.T., Murray, B.W., O'Leary, E.C., Sakata, S.T., Xu, W., Leisten, J.C., Motiwala, A., Pierce, S., Satoh, Y., et al. (2001). SP600125, an anthrapyrazolone inhibitor of Jun N-terminal kinase. Proceedings of the National Academy of Sciences of the United States of America 98, 13681-13686.

Bentink, S., Wessendorf, S., Schwaenen, C., Rosolowski, M., Klapper, W., Rosenwald, A., Ott, G., Banham, A.H., Berger, H., Feller, A.C., et al. (2008). Pathway activation patterns in diffuse large B-cell lymphomas. Leukemia 22, 1746-1754.

Berek, C., Berger, A., and Apel, M. (1991). Maturation of the immune response in germinal centers. Cell 67, 1121-1129.

Bidère, N., Ngo, V.N., Lee, J., Collins, C., Zheng, L., Wan, F., Davis, R.E., Lenz, G., Anderson, D.E., Arnoult, D., et al. (2009). Casein kinase 1alpha governs antigen-receptor-induced NF-kappaB activation and human lymphoma cell survival. Nature 458, 92-96.

Bild, A.H., Yao, G., Chang, J.T., Wang, Q., Potti, A., Chasse, D., Joshi, M.-B., Harpole, D., Lancaster, J.M., Berchuck, A., et al. (2006). Oncogenic pathway signatures in human cancers as a guide to targeted therapies. Nature 439, 353-357.

Birkenkamp, K.U., Tuyt, L.M., Lummen, C., Wierenga, A.T., Kruijer, W., and Vellenga, E. (2000). The p38 MAP kinase inhibitor SB203580 enhances nuclear factor-kappa B transcriptional activity by a non-specific effect upon the ERK pathway. British Journal of Pharmacology 131, 99-107.

Bishop, P.C., Rao, V.K., and Wilson, W.H. (2000). Burkitt's lymphoma: molecular pathogenesis and treatment. Cancer Investigation 18, 574-583.

Blum, K.A., Lozanski, G., and Byrd, J.C. (2004). Adult Burkitt leukemia and lymphoma. Blood 104, 3009-3020.

Van den Bosch, C.A. (2004). Is endemic Burkitt's lymphoma an alliance between three infections and a tumour promoter? The Lancet Oncology 5, 738-746.

Brady, G., Macarthur, G.J., and Farrell, P.J. (2008). Epstein-Barr virus and Burkitt lymphoma. Postgraduate Medical Journal 84, 372-377.

Brocker, T., Gulbranson-Judge, A., Flynn, S., Riedinger, M., Raykundalia, C., and Lane, P. (1999). CD4 T cell traffic control: in vivo evidence that ligation of OX40 on CD4 T cells by OX40-ligand expressed on dendritic cells leads to the accumulation of CD4 T cells in B follicles. European Journal of Immunology 29, 1610-1616.

Buglio, D., Palakurthi, S., Byth, K., Vega, F., Toader, D., Saeh, J., Neelapu, S.S., and Younes, A. (2012). Essential role of TAK1 in regulating mantle cell lymphoma survival. Blood 120, 347-355.

Buonamici, S., Trimarchi, T., Ruocco, M.G., Reavie, L., Cathelin, S., Mar, B.G., Klinakis, A., Lukyanov, Y., Tseng, J.-C., Sen, F., et al. (2009). CCR7 signalling as an essential regulator of CNS infiltration in T-cell leukaemia. Nature 459, 1000-1004. 
Burgering, B.M.T., and Medema, R.H. (2003). Decisions on life and death: FOXO Forkhead transcription factors are in command when PKB/Akt is off duty. Journal of Leukocyte Biology 73, 689701.

Burkitt, D. (1958). A sarcoma involving the jaws in African children. The British Journal of Surgery 46, 218-223.

Calpe, E., Codony, C., Baptista, M.J., Abrisqueta, P., Carpio, C., Purroy, N., Bosch, F., and Crespo, M. (2011). ZAP-70 enhances migration of malignant $B$ lymphocytes toward CCL21 by inducing CCR7 expression via IgM-ERK1/2 activation. Blood 118, 4401-4410.

Cambier, J.C. (1995). New nomenclature for the Reth motif (or ARH1/TAM/ARAM/YXXL). Immunology Today 16, 110.

Cambier, J.C., and Johnson, S.A. (1995). Differential binding activity of ARH1/TAM motifs. Immunology Letters 44, 77-80.

Campo, E., Swerdlow, S.H., Harris, N.L., Pileri, S., Stein, H., and Jaffe, E.S. (2011). The 2008 WHO classification of lymphoid neoplasms and beyond: evolving concepts and practical applications. Blood 117, 5019-5032.

Cancer Research UK (2010). Non-Hodgkin lymphoma incidence statistics. Http://www.cancerresearchuk.org/cancer-info/cancerstats/types/nhl/incidence/uk-nonhodgkinlymphoma-incidence-statistics\#world.

Cargnello, M., and Roux, P.P. (2011). Activation and function of the MAPKs and their substrates, the MAPK-activated protein kinases. Microbiology and Molecular Biology Reviews : MMBR 75, 50-83.

Chalhoub, N., and Baker, S.J. (2009). PTEN and the PI3-kinase pathway in cancer. Annual Review of Pathology 4, 127-150.

Challa, A., Eliopoulos, A.G., Holder, M.J., Burguete, A.S., Pound, J.D., Chamba, A., Grafton, G., Armitage, R.J., Gregory, C.D., Martinez-Valdez, H., et al. (2002). Population depletion activates autonomous CD154-dependent survival in biopsylike Burkitt lymphoma cells. Blood 99, 3411-3418.

Chang, H., Blondal, J.A., Benchimol, S., Minden, M.D., and Messner, H.A. (1995). p53 mutations, cmyc and bcl-2 rearrangements in human non-Hodgkin's lymphoma cell lines. Leukemia \& Lymphoma $19,165-171$.

Chang, H., Li, Q., Moraes, R.C., Lewis, M.T., and Hamel, P.A. (2010). Activation of Erk by sonic hedgehog independent of canonical hedgehog signalling. The International Journal of Biochemistry \& Cell Biology 42, 1462-1471.

Charbonnier, C., Chiquet, J., and Ambroise, C. (2010). Weighted-LASSO for structured network inference from time course data. Statistical Applications in Genetics and Molecular Biology 9, Article 15.

Chen, L., Monti, S., Juszczynski, P., Daley, J., Chen, W., Witzig, T.E., Habermann, T.M., Kutok, J.L., and Shipp, M.A. (2008). SYK-dependent tonic B-cell receptor signaling is a rational treatment target in diffuse large B-cell lymphoma. Blood 111, 2230-2237. 
Cheng, P., Zlobin, A., Volgina, V., Gottipati, S., Osborne, B., Simel, E.J., Miele, L., and Gabrilovich, D.I. (2001). Notch-1 regulates NF-kappaB activity in hemopoietic progenitor cells. Journal of Immunology (Baltimore, Md. : 1950) 167, 4458-4467.

Chiquet, J., Smith, A., Grasseau, G., Matias, C., and Ambroise, C. (2009). SIMoNe: Statistical Inference for MOdular NEtworks. Bioinformatics (Oxford, England) 25, 417-418.

Ci, W., Polo, J.M., and Melnick, A. (2008). B-cell lymphoma 6 and the molecular pathogenesis of diffuse large B-cell lymphoma. Current Opinion in Hematology 15, 381-390.

Ci, W., Polo, J.M., Cerchietti, L., Shaknovich, R., Wang, L., Yang, S.N., Ye, K., Farinha, P., Horsman, D.E., Gascoyne, R.D., et al. (2009). The BCL6 transcriptional program features repression of multiple oncogenes in primary B cells and is deregulated in DLBCL. Blood 113, 5536-5548.

Cooke, E.J., Savage, R.S., Kirk, P.D., Darkins, R., and Wild, D.L. (2011). Bayesian hierarchical clustering for microarray time series data with replicates and outlier measurements. BMC.Bioinformatics. 12, 399-.

Craxton, A., Shu, G., Graves, J.D., Saklatvala, J., Krebs, E.G., and Clark, E.A. (1998). p38 MAPK is required for $C D 40$-induced gene expression and proliferation in $B$ lymphocytes. Journal of Immunology (Baltimore, Md. : 1950) 161, 3225-3236.

Di Cristofano, A., Pesce, B., Cordon-Cardo, C., and Pandolfi, P.P. (1998). Pten is essential for embryonic development and tumour suppression. Nature Genetics 19, 348-355.

Cuadrado, A., and Nebreda, A.R. (2010). Mechanisms and functions of p38 MAPK signalling. The Biochemical Journal 429, 403-417.

Cuenda, A., Rouse, J., Doza, Y.N., Meier, R., Cohen, P., Gallagher, T.F., Young, P.R., and Lee, J.C. (1995). SB 203580 is a specific inhibitor of a MAP kinase homologue which is stimulated by cellular stresses and interleukin-1. FEBS Letters 364, 229-233.

Cultrera, J.L., and Dalia, S.M. (2012). Diffuse large B-cell lymphoma: current strategies and future directions. Cancer Control : Journal of the Moffitt Cancer Center 19, 204-213.

Curnock, A.P., and Knox, K.A. (1998). LY294002-mediated inhibition of phosphatidylinositol 3-kinase activity triggers growth inhibition and apoptosis in CD40-triggered Ramos-Burkitt lymphoma B cells. Cellular Immunology 187, 77-87.

Dal Porto, J.M., Gauld, S.B., Merrell, K.T., Mills, D., Pugh-Bernard, A.E., and Cambier, J. (2004). B cell antigen receptor signaling 101. Molecular Immunology 41, 599-613.

Darkins, R., Cooke, E.J., Ghahramani, Z., Kirk, P.D.W., Wild, D.L., and Savage, R.S. (2013). Accelerating bayesian hierarchical clustering of time series data with a randomised algorithm. PloS One 8, e59795.

Dave, B.J., Nelson, M., Pickering, D.L., Chan, W.C., Greiner, T.C., Weisenburger, D.D., Armitage, J.O., and Sanger, W.G. (2002). Cytogenetic characterization of diffuse large cell lymphoma using multicolor fluorescence in situ hybridization. Cancer Genetics and Cytogenetics 132, 125-132.

Dave, S.S., Fu, K., Wright, G.W., Lam, L.T., Kluin, P., Boerma, E.-J., Greiner, T.C., Weisenburger, D.D., Rosenwald, A., Ott, G., et al. (2006). Molecular diagnosis of Burkitt's lymphoma. The New England Journal of Medicine 354, 2431-2442. 
Davis, R.E., Brown, K.D., Siebenlist, U., and Staudt, L.M. (2001). Constitutive nuclear factor kappaB activity is required for survival of activated B cell-like diffuse large B cell lymphoma cells. The Journal of Experimental Medicine 194, 1861-1874.

Davis, R.E., Ngo, V.N., Lenz, G., Tolar, P., Young, R.M., Romesser, P.B., Kohlhammer, H., Lamy, L., Zhao, H., Yang, Y., et al. (2010). Chronic active B-cell-receptor signalling in diffuse large B-cell lymphoma. Nature 463, 88-92.

Decker, S., Zirlik, K., Djebatchie, L., Hartmann, D., Ihorst, G., Schmitt-Graeff, A., Herchenbach, D., Jumaa, H., Warmuth, M., Veelken, H., et al. (2012). Trisomy 12 and elevated GLI1 and PTCH1 transcript levels are biomarkers for Hedgehog-inhibitor responsiveness in CLL. Blood 119, 997-1007.

DeFranco, A.L. (1997). The complexity of signaling pathways activated by the BCR. Current Opinion in Immunology 9, 296-308.

Delgado, P., Cubelos, B., Calleja, E., Martínez-Martín, N., Ciprés, A., Mérida, I., Bellas, C., Bustelo, X.R., and Alarcón, B. (2009). Essential function for the GTPase TC21 in homeostatic antigen receptor signaling. Nature Immunology 10, 880-888.

Dierks, C., Grbic, J., Zirlik, K., Beigi, R., Englund, N.P., Guo, G.-R., Veelken, H., Engelhardt, M., Mertelsmann, R., Kelleher, J.F., et al. (2007). Essential role of stromally induced hedgehog signaling in B-cell malignancies. Nature Medicine 13, 944-951.

DiSanto, J.P., Bonnefoy, J.Y., Gauchat, J.F., Fischer, A., and de Saint Basile, G. (1993). CD40 ligand mutations in x-linked immunodeficiency with hyper-lgM. Nature 361, 541-543.

Dogan, I., Bertocci, B., Vilmont, V., Delbos, F., Mégret, J., Storck, S., Reynaud, C.-A., and Weill, J.-C. (2009). Multiple layers of B cell memory with different effector functions. Nature Immunology 10 , 1292-1299.

Ear, T., Fortin, C.F., Simard, F. a, and McDonald, P.P. (2010). Constitutive association of TGF-betaactivated kinase 1 with the IkappaB kinase complex in the nucleus and cytoplasm of human neutrophils and its impact on downstream processes. Journal of Immunology (Baltimore, Md. : 1950) $184,3897-3906$.

Egawa, T., Albrecht, B., Favier, B., Sunshine, M.-J., Mirchandani, K., O'Brien, W., Thome, M., and Littman, D.R. (2003). Requirement for CARMA1 in antigen receptor-induced NF-kappa B activation and lymphocyte proliferation. Current Biology : CB 13, 1252-1258.

Elgueta, R., Benson, M.J., de Vries, V.C., Wasiuk, A., Guo, Y., and Noelle, R.J. (2009). Molecular mechanism and function of CD40/CD40L engagement in the immune system. Immunological Reviews $229,152-172$.

Eliopoulos, A.G., Dawson, C.W., Mosialos, G., Floettmann, J.E., Rowe, M., Armitage, R.J., Dawson, J., Zapata, J.M., Kerr, D.J., Wakelam, M.J., et al. (1996). CD40-induced growth inhibition in epithelial cells is mimicked by Epstein-Barr Virus-encoded LMP1: involvement of TRAF3 as a common mediator. Oncogene 13, 2243-2254.

Epstein, A.L., Herman, M.M., Kim, H., Dorfman, R.F., and Kaplan, H.S. (1976). Biology of the human malignant lymphomas. III. Intracranial heterotransplantation in the nude, athymic mouse. Cancer 37, 2158-2176. 
Epstein, A.L., Levy, R., Kim, H., Henle, W., Henle, G., and Kaplan, H.S. (1978). Biology of the human malignant lymphomas. IV. Functional characterization of ten diffuse histiocytic lymphoma cell lines. Cancer 42, 2379-2391.

Fahmi, H., Cochet, C., Hmama, Z., Opolon, P., and Joab, I. (2000). Transforming growth factor beta 1 stimulates expression of the Epstein-Barr virus BZLF1 immediate-early gene product ZEBRA by an indirect mechanism which requires the MAPK kinase pathway. Journal of Virology 74, 5810-5818.

Favata, M.F., Horiuchi, K.Y., Manos, E.J., Daulerio, A.J., Stradley, D.A., Feeser, W.S., Van Dyk, D.E., Pitts, W.J., Earl, R.A., Hobbs, F., et al. (1998). Identification of a novel inhibitor of mitogen-activated protein kinase kinase. The Journal of Biological Chemistry 273, 18623-18632.

Ferlay, J., Parkin, D.M., and Steliarova-Foucher, E. (2010). Estimates of cancer incidence and mortality in Europe in 2008. European Journal of Cancer (Oxford, England : 1990) 46, 765-781.

Ferry, J.A. (2006). Burkitt's lymphoma: clinicopathologic features and differential diagnosis. The Oncologist 11, 375-383.

Feugier, P., Van Hoof, A., Sebban, C., Solal-Celigny, P., Bouabdallah, R., Fermé, C., Christian, B., Lepage, E., Tilly, H., Morschhauser, F., et al. (2005). Long-term results of the R-CHOP study in the treatment of elderly patients with diffuse large B-cell lymphoma: a study by the Groupe d'Etude des Lymphomes de l'Adulte. Journal of Clinical Oncology : Official Journal of the American Society of Clinical Oncology 23, 4117-4126.

Friedberg, J.W. (2011). Relapsed/refractory diffuse large B-cell lymphoma. Hematology / the Education Program of the American Society of Hematology. American Society of Hematology. Education Program 2011, 498-505.

Friedman, N. (2004). Inferring cellular networks using probabilistic graphical models. Science (New York, N.Y.) 303, 799-805.

Fröhlich, H., Fellmann, M., Sueltmann, H., Poustka, A., and Beissbarth, T. (2007). Large scale statistical inference of signaling pathways from RNAi and microarray data. BMC Bioinformatics 8, 386.

Fukuda, T., Yoshida, T., Okada, S., Hatano, M., Miki, T., Ishibashi, K., Okabe, S., Koseki, H., Hirosawa, S., Taniguchi, M., et al. (1997). Disruption of the Bcl6 gene results in an impaired germinal center formation. The Journal of Experimental Medicine 186, 439-448.

Funakoshi, S., Longo, D.L., Beckwith, M., Conley, D.K., Tsarfaty, G., Tsarfaty, I., Armitage, R.J., Fanslow, W.C., Spriggs, M.K., and Murphy, W.J. (1994). Inhibition of human B-cell lymphoma growth by CD40 stimulation. Blood 83, 2787-2794.

Gaide, O., Favier, B., Legler, D.F., Bonnet, D., Brissoni, B., Valitutti, S., Bron, C., Tschopp, J., and Thome, M. (2002). CARMA1 is a critical lipid raft-associated regulator of TCR-induced NF-kappa B activation. Nature Immunology 3, 836-843.

Gallagher, E., Enzler, T., Matsuzawa, A., Anzelon-Mills, A., Otero, D., Holzer, R., Janssen, E., Gao, M., and Karin, M. (2007). Kinase MEKK1 is required for CD40-dependent activation of the kinases Jnk and p38, germinal center formation, B cell proliferation and antibody production. Nature Immunology 8 , $57-63$. 
Gascan, H., Gauchat, J.F., Aversa, G., Van Vlasselaer, P., and de Vries, J.E. (1991). Anti-CD40 monoclonal antibodies or CD4+ T cell clones and IL-4 induce IgG4 and IgE switching in purified human B cells via different signaling pathways. Journal of Immunology (Baltimore, Md. : 1950) 147, 8-13.

Gille, H., Kortenjann, M., Thomae, O., Moomaw, C., Slaughter, C., Cobb, M.H., and Shaw, P.E. (1995). ERK phosphorylation potentiates Elk-1-mediated ternary complex formation and transactivation. The EMBO Journal 14, 951-962.

Gonçalves, J.P., Aires, R.S., Francisco, A.P., and Madeira, S.C. (2012). Regulatory Snapshots: integrative mining of regulatory modules from expression time series and regulatory networks. PloS One 7, e35977.

Goodrich, L. V, and Scott, M.P. (1998). Hedgehog and patched in neural development and disease. Neuron 21, 1243-1257.

Graf, D., Müller, S., Korthäuer, U., van Kooten, C., Weise, C., and Kroczek, R.A. (1995). A soluble form of TRAP (CD40 ligand) is rapidly released after T cell activation. European Journal of Immunology 25, 1749-1754.

Gunvén, P., Klein, G., Klein, E., Norin, T., and Singh, S. (1980). Surface immunoglobulins on Burkitt's lymphoma biopsy cells from 91 patients. International Journal of Cancer. Journal International Du Cancer 25, 711-719.

Hara, H., Wada, T., Bakal, C., Kozieradzki, I., Suzuki, S., Suzuki, N., Nghiem, M., Griffiths, E.K., Krawczyk, C., Bauer, B., et al. (2003). The MAGUK family protein CARD11 is essential for lymphocyte activation. Immunity 18, 763-775.

Haye, A., Albert, J., and Rooman, M. (2012). Robust non-linear differential equation models of gene expression evolution across Drosophila development. BMC Research Notes 5, 46.

Hecht, J.L., and Aster, J.C. (2000). Molecular biology of Burkitt's lymphoma. Journal of Clinical Oncology : Official Journal of the American Society of Clinical Oncology 18, 3707-3721.

Hegde, G. V, Peterson, K.J., Emanuel, K., Mittal, A.K., Joshi, A.D., Dickinson, J.D., Kollessery, G.J., Bociek, R.G., Bierman, P., Vose, J.M., et al. (2008). Hedgehog-induced survival of B-cell chronic lymphocytic leukemia cells in a stromal cell microenvironment: a potential new therapeutic target. Molecular Cancer Research : MCR 6, 1928-1936.

Heisig, J., Weber, D., Englberger, E., Winkler, A., Kneitz, S., Sung, W.-K., Wolf, E., Eilers, M., Wei, C.-L., and Gessler, M. (2012). Target gene analysis by microarrays and chromatin immunoprecipitation identifies HEY proteins as highly redundant bHLH repressors. PLoS Genetics 8, e1002728.

Heller, K.A., and Ghahramani, Z. (2005). Bayesian hierarchical clustering. In Proceedings of the 22nd International Conference on Machine Learning - ICML '05, (New York, New York, USA: ACM Press), pp. 297-304.

Hermann, P., Blanchard, D., de Saint-Vis, B., Fossiez, F., Gaillard, C., Vanbervliet, B., Brière, F., Banchereau, J., and Galizzi, J.P. (1993). Expression of a 32-kDa ligand for the CD40 antigen on activated human T lymphocytes. European Journal of Immunology 23, 961-964.

Hommel, G., and Kropf, S. (2005). Tests for Differentiation in Gene Expression Using a Data-Driven Order or Weights for Hypotheses. Biometrical Journal 47, 554-562. 
Horie, K., Ohashi, M., Satoh, Y., and Sairenji, T. (2007). The role of p38 mitogen-activated protein kinase in regulating interleukin-10 gene expression in Burkitt's lymphoma cell lines. Microbiology and Immunology 51, 149-161.

Horikawa, K., Martin, S.W., Pogue, S.L., Silver, K., Peng, K., Takatsu, K., and Goodnow, C.C. (2007). Enhancement and suppression of signaling by the conserved tail of IgG memory-type B cell antigen receptors. The Journal of Experimental Medicine 204, 759-769.

Hu, Y., Baud, V., Delhase, M., Zhang, P., Deerinck, T., Ellisman, M., Johnson, R., and Karin, M. (1999). Abnormal morphogenesis but intact IKK activation in mice lacking the IKKalpha subunit of IkappaB kinase. Science (New York, N.Y.) 284, 316-320.

Huber, W., von Heydebreck, A., Sultmann, H., Poustka, A., and Vingron, M. (2002). Variance stabilization applied to microarray data calibration and to the quantification of differential expression. Bioinformatics 18, S96-S104.

Hummel, M., Bentink, S., Berger, H., Klapper, W., Wessendorf, S., Barth, T.F.E., Bernd, H.-W., Cogliatti, S.B., Dierlamm, J., Feller, A.C., et al. (2006). A biologic definition of Burkitt's lymphoma from transcriptional and genomic profiling. The New England Journal of Medicine 354, 2419-2430.

Hurlin, P.J., Quéva, C., and Eisenman, R.N. (1997a). Mnt, a novel Max-interacting protein is coexpressed with Myc in proliferating cells and mediates repression at Myc binding sites. Genes \& Development 11, 44-58.

Hurlin, P.J., Qúeva, C., and Eisenman, R.N. (1997b). Mnt: a novel Max-interacting protein and Myc antagonist. Current Topics in Microbiology and Immunology 224, 115-121.

Ikram, M.S., Neill, G.W., Regl, G., Eichberger, T., Frischauf, A.-M., Aberger, F., Quinn, A., and Philpott, M. (2004). GLI2 is expressed in normal human epidermis and BCC and induces GLI1 expression by binding to its promoter. The Journal of Investigative Dermatology 122, 1503-1509.

Incardona, J.P., Gaffield, W., Kapur, R.P., and Roelink, H. (1998). The teratogenic Veratrum alkaloid cyclopamine inhibits sonic hedgehog signal transduction. Development (Cambridge, England) 125, 3553-3562.

Iqbal, J., Gupta, S., Chen, Q.H., Brody, J.P., and Koduru, P. (2007). Diffuse large B-cell lymphoma with a novel translocation involving BCL6. Cancer Genetics and Cytogenetics 178, 73-76.

Irie, T., Muta, T., and Takeshige, K. (2000). TAK1 mediates an activation signal from toll-like receptor(s) to nuclear factor-kappaB in lipopolysaccharide-stimulated macrophages. FEBS Letters $467,160-164$.

Irizarry, R.A. (2003). Summaries of Affymetrix GeneChip probe level data. Nucleic Acids Research 31, $15 \mathrm{e}-15$.

Jacob, J., Kelsoe, G., Rajewsky, K., and Weiss, U. (1991). Intraclonal generation of antibody mutants in germinal centres. Nature 354, 389-392.

Jaffe, E.S. (2009). The 2008 WHO classification of lymphomas: implications for clinical practice and translational research. Hematology / the Education Program of the American Society of Hematology. American Society of Hematology. Education Program 523-531. 
Jaffe, E.S., Harris, N.L., Diebold, J., and Müller-Hermelink, H.K. (1998). World Health Organization Classification of lymphomas: a work in progress. Ann Oncol. 9, -.

Johnson, W.E., Li, C., and Rabinovic, A. (2007). Adjusting batch effects in microarray expression data using empirical Bayes methods. Biostatistics (Oxford, England) 8, 118-127.

Ju, B., Spitsbergen, J., Eden, C.J., Taylor, M.R., and Chen, W. (2009). Co-activation of hedgehog and AKT pathways promote tumorigenesis in zebrafish. Molecular Cancer 8, 40.

Jun, J.E., and Goodnow, C.C. (2003). Scaffolding of antigen receptors for immunogenic versus tolerogenic signaling. Nature Immunology 4, 1057-1064.

Katoh, M., and Katoh, M. (2009). Transcriptional mechanisms of WNT5A based on NF-kappaB, Hedgehog, TGFbeta, and Notch signaling cascades. International Journal of Molecular Medicine 23, 763-769.

Kawabe, T., Naka, T., Yoshida, K., Tanaka, T., Fujiwara, H., Suematsu, S., Yoshida, N., Kishimoto, T., and Kikutani, H. (1994). The immune responses in CD40-deficient mice: impaired immunoglobulin class switching and germinal center formation. Immunity 1, 167-178.

Ke, J., Gururajan, M., Kumar, A., Simmons, A., Turcios, L., Chelvarajan, R.L., Cohen, D.M., Wiest, D.L., Monroe, J.G., and Bondada, S. (2006). The role of MAPKs in B cell receptor-induced down-regulation of Egr-1 in immature B lymphoma cells. The Journal of Biological Chemistry 281, 39806-39818.

Klapproth, K., Sander, S., Marinkovic, D., Baumann, B., and Wirth, T. (2009). The IKK2/NF-\{kappa\}B pathway suppresses MYC-induced lymphomagenesis. Blood 114, 2448-2458.

Klein, U., and Dalla-Favera, R. (2008). Germinal centres: role in B-cell physiology and malignancy. Nature Reviews. Immunology 8, 22-33.

Klein, G., Giovanella, B., Westman, A., Stehlin, J.S., and Mumford, D. (1975). An EBV-genomenegative cell line established from an American Burkitt lymphoma; receptor characteristics. EBV infectibility and permanent conversion into EBV-positive sublines by in vitro infection. Intervirology 5, 319-334.

Kloo, B., Nagel, D., Pfeifer, M., Grau, M., Düwel, M., Vincendeau, M., Dörken, B., Lenz, P., Lenz, G., and Krappmann, D. (2011). Critical role of PI3K signaling for NF-kappaB-dependent survival in a subset of activated B-cell-like diffuse large B-cell lymphoma cells. Proceedings of the National Academy of Sciences of the United States of America 108, 272-277.

Van Kooten, C., and Banchereau, J. (2000). CD40-CD40 ligand. Journal of Leukocyte Biology 67, 2-17.

Kopp, F., Stratton, C.F., Akella, L.B., and Tan, D.S. (2012). A diversity-oriented synthesis approach to macrocycles via oxidative ring expansion. Nature Chemical Biology 8, 358-365.

Kramer, M.H., Hermans, J., Wijburg, E., Philippo, K., Geelen, E., van Krieken, J.H., de Jong, D., Maartense, E., Schuuring, E., and Kluin, P.M. (1998). Clinical relevance of BCL2, BCL6, and MYC rearrangements in diffuse large B-cell lymphoma. Blood 92, 3152-3162.

Kraus, M., Alimzhanov, M.B., Rajewsky, N., and Rajewsky, K. (2004). Survival of resting mature B lymphocytes depends on BCR signaling via the Igalpha/beta heterodimer. Cell 117, 787-800. 
Kube, D., Platzer, C., von Knethen, A., Straub, H., Bohlen, H., Hafner, M., and Tesch, H. (1995). Isolation of the human interleukin 10 promoter. Characterization of the promoter activity in Burkitt's lymphoma cell lines. Cytokine 7, 1-7.

Küppers, R. (2005). Mechanisms of B-cell lymphoma pathogenesis. Nature Reviews. Cancer 5, 251262.

Küppers, R., and Dalla-Favera, R. (2001). Mechanisms of chromosomal translocations in B cell lymphomas. Oncogene 20, 5580-5594.

Küppers, R., Klein, U., Hansmann, M.L., and Rajewsky, K. (1999). Cellular origin of human B-cell lymphomas. The New England Journal of Medicine 341, 1520-1529.

Kusam, S., Munugalavadla, V., Sawant, D., and Dent, A. (2009). BCL6 cooperates with CD40 stimulation and loss of p53 function to rapidly transform primary B cells. International Journal of Cancer. Journal International Du Cancer 125, 977-981.

Kutz, H., Reisbach, G., Schultheiss, U., and Kieser, A. (2008). The c-Jun N-terminal kinase pathway is critical for cell transformation by the latent membrane protein 1 of Epstein-Barr virus. Virology 371, 246-256.

Laemmli, U.K. (1970). Cleavage of structural proteins during the assembly of the head of bacteriophage T4. Nature 227, 680-685.

Lam, K.P., Kühn, R., and Rajewsky, K. (1997). In vivo ablation of surface immunoglobulin on mature B cells by inducible gene targeting results in rapid cell death. Cell 90, 1073-1083.

Lam, L.T., Davis, R.E., Pierce, J., Hepperle, M., Xu, Y., Hottelet, M., Nong, Y., Wen, D., Adams, J., Dang, L., et al. (2005). Small molecule inhibitors of IkappaB kinase are selectively toxic for subgroups of diffuse large B-cell lymphoma defined by gene expression profiling. Clinical Cancer Research : an Official Journal of the American Association for Cancer Research 11, 28-40.

Lam, L.T., Wright, G., Davis, R.E., Lenz, G., Farinha, P., Dang, L., Chan, J.W., Rosenwald, A., Gascoyne, R.D., and Staudt, L.M. (2008). Cooperative signaling through the signal transducer and activator of transcription 3 and nuclear factor-\{kappa\}B pathways in subtypes of diffuse large B-cell lymphoma. Blood 111, 3701-3713.

Lane, P., Traunecker, A., Hubele, S., Inui, S., Lanzavecchia, A., and Gray, D. (1992). Activated human T cells express a ligand for the human B cell-associated antigen CD40 which participates in T celldependent activation of B lymphocytes. European Journal of Immunology 22, 2573-2578.

Läuter, J., Glimm, E., and Kropf, S. (1996). New Tests for Data with an Inherent Structure. Biometrical Journal 38, 1-23.

Läuter, J., Glimm, E., and Kropf, S. (1998). Multivariate tests based on left-spherically distributed linear scores. The Annals of Statistics 26, 1972-1988.

Läuter, J., Rosolowski, M., and Glimm, E. (2012). Exact Multivariate Tests - A New Effective Principle of Controlled Model Choice. 18.

Lee, W.-P., and Tzou, W.-S. (2009). Computational methods for discovering gene networks from expression data. Briefings in Bioinformatics 10, 408-423. 
Lee, J., Platt, K.A., Censullo, P., and Ruiz i Altaba, A. (1997). Gli1 is a target of Sonic hedgehog that induces ventral neural tube development. Development (Cambridge, England) 124, 2537-2552.

Lee, J.C., Laydon, J.T., McDonnell, P.C., Gallagher, T.F., Kumar, S., Green, D., McNulty, D., Blumenthal, M.J., Heys, J.R., and Landvatter, S.W. (1994). A protein kinase involved in the regulation of inflammatory cytokine biosynthesis. Nature 372, 739-746.

Lee, J.K.J., Mathew, S.O.S., Vaidya, S. V, Kumaresan, P.R., and Mathew, P.A. (2007). CS1 (CRACC, $\mathrm{CD} 319)$ induces proliferation and autocrine cytokine expression on human $B$ lymphocytes. The Journal of ... 179, 4672-4678.

Lenoir, G.M., Vuillaume, M., and Bonnardel, C. (1985). The use of lymphomatous and lymphoblastoid cell lines in the study of Burkitt's lymphoma. IARC Sci.Publ. 309-318.

Lenz, G., Wright, G.W., Emre, N.C.T., Kohlhammer, H., Dave, S.S., Davis, R.E., Carty, S., Lam, L.T., Shaffer, a L., Xiao, W., et al. (2008). Molecular subtypes of diffuse large B-cell lymphoma arise by distinct genetic pathways. Proceedings of the National Academy of Sciences of the United States of America 105, 13520-13525.

Li, Q., and Verma, I.M. (2002). NF-kappaB regulation in the immune system. Nature Reviews. Immunology 2, 725-734.

Li, Z.W., Chu, W., Hu, Y., Delhase, M., Deerinck, T., Ellisman, M., Johnson, R., and Karin, M. (1999). The IKKbeta subunit of IkappaB kinase (IKK) is essential for nuclear factor kappaB activation and prevention of apoptosis. The Journal of Experimental Medicine 189, 1839-1845.

Lin, T.L., Wang, Q.H., Brown, P., Peacock, C., Merchant, A. a, Brennan, S., Jones, E., McGovern, K., Watkins, D.N., Sakamoto, K.M., et al. (2010). Self-renewal of acute lymphocytic leukemia cells is limited by the Hedgehog pathway inhibitors cyclopamine and IPI-926. PloS One 5, e15262.

Lindemann, R.K. (2008). Stroma-initiated hedgehog signaling takes center stage in B-cell lymphoma. Cancer Research 68, 961-964.

Lohr, J.G., Stojanov, P., Lawrence, M.S., Auclair, D., Chapuy, B., Sougnez, C., Cruz-Gordillo, P., Knoechel, B., Asmann, Y.W., Slager, S.L., et al. (2012). Discovery and prioritization of somatic mutations in diffuse large B-cell lymphoma (DLBCL) by whole-exome sequencing. Proceedings of the National Academy of Sciences of the United States of America 109, 3879-3884.

Van der Lugt, N.M., Domen, J., Verhoeven, E., Linders, K., van der Gulden, H., Allen, J., and Berns, A. (1995). Proviral tagging in E mu-myc transgenic mice lacking the Pim-1 proto-oncogene leads to compensatory activation of Pim-2. The EMBO Journal 14, 2536-2544.

MacLennan, I.C. (1994). Germinal centers. Annual Review of Immunology 12, 117-139.

Madhala-Levy, D., Williams, V.C., Hughes, S.M., Reshef, R., and Halevy, O. (2012). Cooperation between Shh and IGF-I in promoting myogenic proliferation and differentiation via the MAPK/ERK and PI3K/Akt pathways requires Smo activity. Journal of Cellular Physiology 227, 1455-1464.

Magrath, I., Adde, M., Shad, A., Venzon, D., Seibel, N., Gootenberg, J., Neely, J., Arndt, C., Nieder, M., Jaffe, E., et al. (1996). Adults and children with small non-cleaved-cell lymphoma have a similar excellent outcome when treated with the same chemotherapy regimen. Journal of Clinical Oncology: Official Journal of the American Society of Clinical Oncology 14, 925-934. 
Mangelberger, D., Kern, D., Loipetzberger, A., Eberl, M., and Aberger, F. (2012). Cooperative Hedgehog-EGFR signaling. Frontiers in Bioscience : a Journal and Virtual Library 17, 90-99.

Mar, B.G., Amakye, D., Aifantis, I., and Buonamici, S. (2011). The controversial role of the Hedgehog pathway in normal and malignant hematopoiesis. Leukemia 25, 1665-1673.

Marigo, V., and Tabin, C.J. (1996). Regulation of patched by sonic hedgehog in the developing neural tube. Proceedings of the National Academy of Sciences of the United States of America 93, 93469351.

Marino, S. (2005). Medulloblastoma: developmental mechanisms out of control. Trends in Molecular Medicine 11, 17-22.

Markman, B., Atzori, F., Pérez-García, J., Tabernero, J., and Baselga, J. (2010). Status of PI3K inhibition and biomarker development in cancer therapeutics. Annals of Oncology : Official Journal of the European Society for Medical Oncology / ESMO 21, 683-691.

Markowetz, F., and Spang, R. (2007). Inferring cellular networks--a review. BMC Bioinformatics 8 Suppl 6, S5.

Markowetz, F., Bloch, J., and Spang, R. (2005). Non-transcriptional pathway features reconstructed from secondary effects of RNA interference. Bioinformatics (Oxford, England) 21, 4026-4032.

Markowetz, F., Kostka, D., Troyanskaya, O.G., and Spang, R. (2007). Nested effects models for highdimensional phenotyping screens. Bioinformatics (Oxford, England) 23, i305-12.

Martin, S.W., and Goodnow, C.C. (2002). Burst-enhancing role of the IgG membrane tail as a molecular determinant of memory. Nature Immunology 3, 182-188.

De Matos Simoes, R., Tripathi, S., and Emmert-Streib, F. (2012). Organizational structure and the periphery of the gene regulatory network in B-cell lymphoma. BMC Systems Biology 6, 38.

Matsuzawa, A., Tseng, P.-H., Vallabhapurapu, S., Luo, J.-L., Zhang, W., Wang, H., Vignali, D.A.A., Gallagher, E., and Karin, M. (2008). Essential cytoplasmic translocation of a cytokine receptorassembled signaling complex. Science (New York, N.Y.) 321, 663-668.

Mazzei, G.J., Edgerton, M.D., Losberger, C., Lecoanet-Henchoz, S., Graber, P., Durandy, A., Gauchat, J.F., Bernard, A., Allet, B., and Bonnefoy, J.Y. (1995). Recombinant soluble trimeric CD40 ligand is biologically active. The Journal of Biological Chemistry 270, 7025-7028.

Mead, G.M., Sydes, M.R., Walewski, J., Grigg, A., Hatton, C.S., Pescosta, N., Guarnaccia, C., Lewis, M.S., McKendrick, J., Stenning, S.P., et al. (2002). An international evaluation of CODOX-M and CODOX-M alternating with IVAC in adult Burkitt's lymphoma: results of United Kingdom Lymphoma Group LY06 study. Annals of Oncology : Official Journal of the European Society for Medical Oncology / ESMO 13, 1264-1274.

Mizuarai, S., Kawagishi, A., and Kotani, H. (2009). Inhibition of p70S6K2 down-regulates Hedgehog/GLI pathway in non-small cell lung cancer cell lines. Molecular Cancer 8, 44.

Mogami, H., Mills, C., and Gallacher, D. (1997). inhibitor, U73122, releases intracellular Ca2+, potentiates Ins $(1,4,5) \mathrm{P} 3$-mediated $\mathrm{Ca} 2+$ release and directly activates ion channels in mouse pancreatic acinar cells. Biochemical Journal 324 ( Pt 2, 645-651. 
Monroe, J.G. (2006). ITAM-mediated tonic signalling through pre-BCR and BCR complexes. Nature Reviews. Immunology 6, 283-294.

Murata, T., Shimada, M., Sakakibara, S., Yoshino, T., Kadono, H., Masuda, T., Shimazaki, M., Shintani, T., Fuchikami, K., Sakai, K., et al. (2003). Discovery of novel and selective IKK-beta serine-threonine protein kinase inhibitors. Part 1. Bioorganic \& Medicinal Chemistry Letters 13, 913-918.

Nascimento, M., Sáfadi, T., Fonseca e Silva, F., and Nascimento, A.C.C. (2012). Bayesian model-based clustering of temporal gene expression using autoregressive panel data approach. Bioinformatics (Oxford, England) 28, 2004-2007.

Newton, K., and Dixit, V.M. (2003). Mice lacking the CARD of CARMA1 exhibit defective B lymphocyte development and impaired proliferation of their B and T lymphocytes. Current Biology : CB 13, 12471251.

Ngo, V.N., Davis, R.E., Lamy, L., Yu, X., Zhao, H., Lenz, G., Lam, L.T., Dave, S., Yang, L., Powell, J., et al. (2006). A loss-of-function RNA interference screen for molecular targets in cancer. Nature 441, 106110.

Nilsson, K., and Pontén, J. (1975). Classification and biological nature of established human hematopoietic cell lines. International Journal of Cancer. Journal International Du Cancer 15, 321341.

Ninomiya-Tsuji, J., Kishimoto, K., Hiyama, A., Inoue, J., Cao, Z., and Matsumoto, K. (1999). The kinase TAK1 can activate the NIK-I kappaB as well as the MAP kinase cascade in the IL-1 signalling pathway. Nature 398, 252-256.

Ninomiya-Tsuji, J., Kajino, T., Ono, K., Ohtomo, T., Matsumoto, M., Shiina, M., Mihara, M., Tsuchiya, M., and Matsumoto, K. (2003). A resorcylic acid lactone, 5Z-7-oxozeaenol, prevents inflammation by inhibiting the catalytic activity of TAK1 MAPK kinase kinase. The Journal of Biological Chemistry 278, 18485-18490.

Nishimura, M., Shin, M.-S., Singhirunnusorn, P., Suzuki, S., Kawanishi, M., Koizumi, K., Saiki, I., and Sakurai, H. (2009). TAK1-mediated serine/threonine phosphorylation of epidermal growth factor receptor via p38/extracellular signal-regulated kinase: NF-\{kappa\}B-independent survival pathways in tumor necrosis factor alpha signaling. Molecular and Cellular Biology 29, 5529-5539.

Nussenzweig, A., and Nussenzweig, M.C. (2010). Origin of chromosomal translocations in lymphoid cancer. Cell 141, 27-38.

Nüsslein-Volhard, C., and Wieschaus, E. (1980). Mutations affecting segment number and polarity in Drosophila. Nature 287, 795-801.

Ohori, M., Kinoshita, T., Yoshimura, S., Warizaya, M., Nakajima, H., and Miyake, H. (2007). Role of a cysteine residue in the active site of ERK and the MAPKK family. Biochemical and Biophysical Research Communications 353, 633-637.

Ok, C.Y., Singh, R.R., and Vega, F. (2012). Aberrant activation of the hedgehog signaling pathway in malignant hematological neoplasms. The American Journal of Pathology 180, 2-11. 
Okuyama, K., Murata, A., Sudo, T., Yoshino, M., and Hayashi, S.-I. (2012). A checkpoint in Blymphopoiesis related to Notch resistance. Biochemical and Biophysical Research Communications 417, 141-146.

Oliveros, J.C. (2007). VENNY. An interactive tool for comparing lists with Venn Diagrams.

Parekh, S., Polo, J.M., Shaknovich, R., Juszczynski, P., Lev, P., Ranuncolo, S.M., Yin, Y., Klein, U., Cattoretti, G., Dalla Favera, R., et al. (2007). BCL6 programs lymphoma cells for survival and differentiation through distinct biochemical mechanisms. Blood 110, 2067-2074.

Pattle, S.B., and Farrell, P.J. (2006). The role of Epstein-Barr virus in cancer. Expert Opinion on Biological Therapy 6, 1193-1205.

Pearson, G. (2001). Mitogen-Activated Protein (MAP) Kinase Pathways: Regulation and Physiological Functions. Endocrine Reviews 22, 153-183.

Pellat-Deceunynck, C., Amiot, M., Robillard, N., Wijdenes, J., and Bataille, R. (1996). CD11a-CD18 and CD102 interactions mediate human myeloma cell growth arrest induced by CD40 stimulation. Cancer Research 56, 1909-1916.

Perkins, A.S., and Friedberg, J.W. (2008). Burkitt lymphoma in adults. Hematology / the Education Program of the American Society of Hematology. American Society of Hematology. Education Program 341-348.

Pham, L. V, Tamayo, A.T., Yoshimura, L.C., Lo, P., Terry, N., Reid, P.S., and Ford, R.J. (2002). A CD40 Signalosome anchored in lipid rafts leads to constitutive activation of NF-kappaB and autonomous cell growth in B cell lymphomas. Immunity 16, 37-50.

Pogue, S.L., Kurosaki, T., Bolen, J., and Herbst, R. (2000). B cell antigen receptor-induced activation of Akt promotes B cell survival and is dependent on Syk kinase. Journal of Immunology (Baltimore, Md. : 1950) $165,1300-1306$.

Polo, J.M., Dell'Oso, T., Ranuncolo, S.M., Cerchietti, L., Beck, D., Da Silva, G.F., Prive, G.G., Licht, J.D., and Melnick, A. (2004). Specific peptide interference reveals BCL6 transcriptional and oncogenic mechanisms in B-cell lymphoma cells. Nature Medicine 10, 1329-1335.

Polo, J.M., Juszczynski, P., Monti, S., Cerchietti, L., Ye, K., Greally, J.M., Shipp, M., and Melnick, A. (2007). Transcriptional signature with differential expression of BCL6 target genes accurately identifies BCL6-dependent diffuse large B cell lymphomas. Proceedings of the National Academy of Sciences of the United States of America 104, 3207-3212.

Powles, T., Matthews, G., and Bower, M. (2000). AIDS related systemic non-Hodgkin's lymphoma. Sexually Transmitted Infections 76, 335-341.

Pui, J.C., Allman, D., Xu, L., DeRocco, S., Karnell, F.G., Bakkour, S., Lee, J.Y., Kadesch, T., Hardy, R.R., Aster, J.C., et al. (1999). Notch1 expression in early lymphopoiesis influences B versus $T$ lineage determination. Immunity 11, 299-308.

Qu, C., Liu, Y., Kunkalla, K., Singh, R.R., Blonska, M., Lin, X., Agarwal, N.K., and Vega, F. (2013). Trimeric $G$ protein-CARMA1 axis links smoothened, the hedgehog receptor transducer, to NF-KB activation in diffuse large B-cell lymphoma. Blood 4718-4728. 
Radtke, F., Wilson, A., and MacDonald, H.R. (2004). Notch signaling in T- and B-cell development. Current Opinion in Immunology 16, 174-179.

Rakowski, L.A., Garagiola, D.D., Li, C.M., Decker, M., Caruso, S., Jones, M., Kuick, R., Cierpicki, T., Maillard, I., and Chiang, M.Y. (2013). Convergence of the ZMIZ1 and NOTCH1 pathways at C-MYC in acute T lymphoblastic leukemias. Cancer Research 73, 930-941.

Raman, M., Chen, W., and Cobb, M.H. (2007). Differential regulation and properties of MAPKs. Oncogene 26, 3100-3112.

Ramirez, E., Singh, R.R., Kunkalla, K., Liu, Y., Qu, C., Cain, C., Multani, A.S., Lennon, P.A., Jackacky, J., Ho, M., et al. (2012). Defining causative factors contributing in the activation of hedgehog signaling in diffuse large B-cell lymphoma. Leukemia Research 36, 1267-1273.

Rao, G., Pedone, C.A., Coffin, C.M., Holland, E.C., and Fults, D.W. c-Myc enhances sonic hedgehoginduced medulloblastoma formation from nestin-expressing neural progenitors in mice. Neoplasia (New York, N.Y.) 5, 198-204.

Rehm, A., Mensen, A., Schradi, K., Gerlach, K., Wittstock, S., Winter, S., Büchner, G., Dörken, B., Lipp, M., and Höpken, U.E. (2011). Cooperative function of CCR7 and lymphotoxin in the formation of a lymphoma-permissive niche within murine secondary lymphoid organs. Blood 118, 1020-1033.

Reth, M. (1989). Antigen receptor tail clue. Nature 338, 383-384.

Reya, T., O'Riordan, M., Okamura, R., Devaney, E., Willert, K., Nusse, R., and Grosschedl, R. (2000). Wnt signaling regulates $B$ lymphocyte proliferation through a LEF-1 dependent mechanism. Immunity $13,15-24$.

Richards, J.D., Gold, M.R., Hourihane, S.L., DeFranco, A.L., and Matsuuchi, L. (1996). Reconstitution of $B$ cell antigen receptor-induced signaling events in a nonlymphoid cell line by expressing the Syk protein-tyrosine kinase. The Journal of Biological Chemistry 271, 6458-6466.

Richards, J.D., Davé, S.H., Chou, C.H., Mamchak, a a, and DeFranco, a L. (2001). Inhibition of the MEK/ERK signaling pathway blocks a subset of $B$ cell responses to antigen. Journal of Immunology (Baltimore, Md. : 1950) 166, 3855-3864.

Rosenwald, A., Wright, G., Chan, W.C., Connors, J.M., Campo, E., Fisher, R.I., Gascoyne, R.D., MullerHermelink, H.K., Smeland, E.B., Giltnane, J.M., et al. (2002). The use of molecular profiling to predict survival after chemotherapy for diffuse large-B-cell lymphoma. The New England Journal of Medicine 346, 1937-1947.

Rowley, R.B., Burkhardt, A.L., Chao, H.G., Matsueda, G.R., and Bolen, J.B. (1995). Syk protein-tyrosine kinase is regulated by tyrosine-phosphorylated Ig alpha/Ig beta immunoreceptor tyrosine activation motif binding and autophosphorylation. The Journal of Biological Chemistry 270, 11590-11594.

Ruefli-Brasse, A.A., French, D.M., and Dixit, V.M. (2003). Regulation of NF-kappaB-dependent lymphocyte activation and development by paracaspase. Science (New York, N.Y.) 302, 1581-1584.

Ruland, J., and Mak, T.W. (2003). Transducing signals from antigen receptors to nuclear factor kappaB. Immunological Reviews 193, 93-100. 
Ruland, J., Duncan, G.S., Elia, A., del Barco Barrantes, I., Nguyen, L., Plyte, S., Millar, D.G., Bouchard, D., Wakeham, A., Ohashi, P.S., et al. (2001). Bcl10 is a positive regulator of antigen receptor-induced activation of NF-kappaB and neural tube closure. Cell 104, 33-42.

Ruland, J., Duncan, G.S., Wakeham, A., and Mak, T.W. (2003). Differential requirement for Malt1 in T and $B$ cell antigen receptor signaling. Immunity $19,749-758$.

Sacedón, R., Díez, B., Nuñez, V., Hernández-López, C., Gutierrez-Frías, C., Cejalvo, T., Outram, S. V, Crompton, T., Zapata, A.G., Vicente, A., et al. (2005). Sonic hedgehog is produced by follicular dendritic cells and protects germinal center B cells from apoptosis. J.Immunol. 174, 1456-1461.

Saijo, K., Schmedt, C., Su, I.-H., Karasuyama, H., Lowell, C.A., Reth, M., Adachi, T., Patke, A., Santana, A., and Tarakhovsky, A. (2003). Essential role of Src-family protein tyrosine kinases in NF-kappaB activation during B cell development. Nature Immunology 4, 274-279.

Saito, M., Novak, U., Piovan, E., Basso, K., Sumazin, P., Schneider, C., Crespo, M., Shen, Q., Bhagat, G., Califano, A., et al. (2009). BCL6 suppression of BCL2 via Miz1 and its disruption in diffuse large B cell lymphoma. Proceedings of the National Academy of Sciences of the United States of America 106, 11294-11299.

Salmena, L., Carracedo, A., and Pandolfi, P.P. (2008). Tenets of PTEN Tumor Suppression. Cell 133, 403-414.

Sanda, T., lida, S., Ogura, H., and Asamitsu, K. (2005). Growth inhibition of multiple myeloma cells by a novel IKB kinase inhibitor. Clinical Cancer ... 11, 1974-1982.

Sander, S., Calado, D.P., Srinivasan, L., Köchert, K., Zhang, B., Rosolowski, M., Rodig, S.J., Holzmann, K., Stilgenbauer, S., Siebert, R., et al. (2012). Synergy between PI3K signaling and MYC in Burkitt lymphomagenesis. Cancer Cell 22, 167-179.

Sarbassov, D.D., Guertin, D.A., Ali, S.M., and Sabatini, D.M. (2005). Phosphorylation and regulation of Akt/PKB by the rictor-mTOR complex. Science (New York, N.Y.) 307, 1098-1101.

Sato, S., Sanjo, H., Takeda, K., Ninomiya-Tsuji, J., Yamamoto, M., Kawai, T., Matsumoto, K., Takeuchi, O., and Akira, S. (2005). Essential function for the kinase TAK1 in innate and adaptive immune responses. Nature Immunology 6, 1087-1095.

Savage, R.S., Heller, K., Xu, Y., Ghahramani, Z., Truman, W.M., Grant, M., Denby, K.J., and Wild, D.L. (2009). R/BHC: fast Bayesian hierarchical clustering for microarray data. BMC Bioinformatics 10, 242.

Schirmer, A., Kennedy, J., Murli, S., Reid, R., and Santi, D. V (2006). Targeted covalent inactivation of protein kinases by resorcylic acid lactone polyketides. Proceedings of the National Academy of Sciences of the United States of America 103, 4234-4239.

Schmitz, R., Stanelle, J., Hansmann, M.-L., and Küppers, R. (2009). Pathogenesis of classical and lymphocyte-predominant Hodgkin lymphoma. Annual Review of Pathology 4, 151-174.

Schmitz, R., Young, R.M., Ceribelli, M., Jhavar, S., Xiao, W., Zhang, M., Wright, G., Shaffer, A.L., Hodson, D.J., Buras, E., et al. (2012). Burkitt lymphoma pathogenesis and therapeutic targets from structural and functional genomics. Nature 490, 116-120. 
Schnidar, H., Eberl, M., Klingler, S., Mangelberger, D., Kasper, M., Hauser-Kronberger, C., Regl, G., Kroismayr, R., Moriggl, R., Sibilia, M., et al. (2009). Epidermal growth factor receptor signaling synergizes with Hedgehog/GLI in oncogenic transformation via activation of the MEK/ERK/JUN pathway. Cancer Research 69, 1284-1292.

Schrader, A. (2011). Identification and functional characterization of oncogenic pathway signatures in malignant lymphoma. Georg-August University Göttingen.

Schrader, A., Bentink, S., Spang, R., Lenze, D., Hummel, M., Kuo, M., Arrand, J.R., Murray, P.G., Trümper, L., Kube, D., et al. (2012a). High Myc activity is an independent negative prognostic factor for diffuse large B cell lymphomas. International Journal of Cancer. Journal International Du Cancer 131, E348-61.

Schrader, A., Meyer, K., von Bonin, F., Vockerodt, M., Walther, N., Hand, E., Ulrich, A., Matulewicz, K., Lenze, D., Hummel, M., et al. (2012b). Global gene expression changes of in vitro stimulated human transformed germinal centre B cells as surrogate for oncogenic pathway activation in individual aggressive B cell lymphomas. Cell Communication and Signaling : CCS 10, 43.

Schrantz, N., Bourgeade, M.F., Mouhamad, S., Leca, G., Sharma, S., and Vazquez, A. (2001). p38mediated regulation of an Fas-associated death domain protein-independent pathway leading to caspase-8 activation during TGFbeta-induced apoptosis in human Burkitt lymphoma B cells BL41. Molecular Biology of the Cell 12, 3139-3151.

Schreiber, E., Matthias, P., Müller, M.M., and Schaffner, W. (1989). Rapid detection of octamer binding proteins with "mini-extracts", prepared from a small number of cells. Nucleic Acids Research 17,6419 .

Schulz, M.H., Devanny, W.E., Gitter, A., Zhong, S., Ernst, J., and Bar-Joseph, Z. (2012). DREM 2.0: Improved reconstruction of dynamic regulatory networks from time-series expression data. BMC Systems Biology 6, 104.

Schuman, J., Chen, Y., Podd, A., Yu, M., Liu, H.-H., Wen, R., Chen, Z.J., and Wang, D. (2009). A critical role of TAK1 in B-cell receptor-mediated nuclear factor kappaB activation. Blood 113, 4566-4574.

Schwanbeck, R., and Just, U. (2011). The Notch signaling pathway in hematopoiesis and hematologic malignancies. Haematologica 96, 1735-1737.

Segal, G.H., Edinger, M.G., Owen, M., McNealis, M., Lopez, P., Perkins, A., Linden, M.D., Fishleder, A.J., Stoler, M.H., and Tubbs, R.R. (1991). Concomitant delineation of surface Ig, B-cell differentiation antigens, and HLADR on lymphoid proliferations using three-color immunocytometry. Cytometry 12 , 350-359.

Senftleben, U., Cao, Y., Xiao, G., Greten, F.R., Krähn, G., Bonizzi, G., Chen, Y., Hu, Y., Fong, A., Sun, S.C., et al. (2001). Activation by IKKalpha of a second, evolutionary conserved, NF-kappa B signaling pathway. Science (New York, N.Y.) 293, 1495-1499.

Shinohara, H., and Kurosaki, T. (2009). Comprehending the complex connection between PKCbeta, TAK1, and IKK in BCR signaling. Immunological Reviews 232, 300-318.

Shinohara, H., Yasuda, T., Aiba, Y., Sanjo, H., Hamadate, M., Watarai, H., Sakurai, H., and Kurosaki, T. (2005). PKC beta regulates BCR-mediated IKK activation by facilitating the interaction between TAK1 and CARMA1. The Journal of Experimental Medicine 202, 1423-1431. 
Shirogane, T., Fukada, T., Muller, J.M., Shima, D.T., Hibi, M., and Hirano, T. (1999). Synergistic roles for Pim-1 and c-Myc in STAT3-mediated cell cycle progression and antiapoptosis. Immunity 11, 709719.

Shkoda, A., Town, J. a, Griese, J., Romio, M., Sarioglu, H., Knöfel, T., Giehler, F., Kieser, A., and Knofel, T. (2012). The germinal center kinase TNIK is required for canonical NF-kappaB and JNK signaling in Bcells by the EBV oncoprotein LMP1 and the CD40 receptor. PLoS.Biol. 10, e1001376-.

Siggins, S.L., Nguyen, N.-Y.N., McCormack, M.P., Vasudevan, S., Villani, R., Jane, S.M., Wainwright, B.J., and Curtis, D.J. (2009). The Hedgehog receptor Patched1 regulates myeloid and lymphoid progenitors by distinct cell-extrinsic mechanisms. Blood 114, 995-1004.

Singh, R.R., Cho-Vega, J.H., Davuluri, Y., Ma, S., Kasbidi, F., Milito, C., Lennon, P.A., Drakos, E., Medeiros, L.J., Luthra, R., et al. (2009). Sonic hedgehog signaling pathway is activated in ALK-positive anaplastic large cell lymphoma. Cancer Research 69, 2550-2558.

Singh, R.R., Kim, J.E., Davuluri, Y., Drakos, E., Cho-Vega, J.H., Amin, H.M., and Vega, F. (2010). Hedgehog signaling pathway is activated in diffuse large B-cell lymphoma and contributes to tumor cell survival and proliferation. Leukemia 24, 1025-1036.

Singh, R.R., Kunkalla, K., Qu, C., Schlette, E., Neelapu, S.S., Samaniego, F., and Vega, F. (2011). ABCG2 is a direct transcriptional target of hedgehog signaling and involved in stroma-induced drug tolerance in diffuse large B-cell lymphoma. Oncogene 30, 4874-4886.

Smyth, G.K. (2005). Limma: linear models for microarray data. In Bioinformatics and Computational Biology Solutions Using R and Bioconductor, R. Gentleman, V.J. Carey, W. Huber, R.A. Irizarry, and S. Dudoit, eds. (New York: Springer-Verlag), pp. 397-420.

Smyth, G.K., Michaud, J., and Scott, H.S. (2005). Use of within-array replicate spots for assessing differential expression in microarray experiments. Bioinformatics (Oxford, England) 21, 2067-2075.

Sommer, A., Bousset, K., Kremmer, E., Austen, M., and Lüscher, B. (1998). Identification and characterization of specific DNA-binding complexes containing members of the Myc/Max/Mad network of transcriptional regulators. The Journal of Biological Chemistry 273, 6632-6642.

Sorrentino, A., Thakur, N., Grimsby, S., Marcusson, A., von Bulow, V., Schuster, N., Zhang, S., Heldin, C.-H., and Landström, M. (2008). The type I TGF-beta receptor engages TRAF6 to activate TAK1 in a receptor kinase-independent manner. Nature Cell Biology 10, 1199-1207.

Spriggs, M.K., Armitage, R.J., Strockbine, L., Clifford, K.N., Macduff, B.M., Sato, T.A., Maliszewski, C.R., and Fanslow, W.C. (1992). Recombinant human CD40 ligand stimulates B cell proliferation and immunoglobulin E secretion. The Journal of Experimental Medicine 176, 1543-1550.

Stevenson, F.K., Sahota, S.S., Ottensmeier, C.H., Zhu, D., Forconi, F., and Hamblin, T.J. (2001). The occurrence and significance of $\mathrm{V}$ gene mutations in $\mathrm{B}$ cell-derived human malignancy. Advances in Cancer Research 83, 81-116.

$\mathrm{Su}$, C., Andrew, A., Karagas, M.R., and Borsuk, M.E. (2013). Using Bayesian networks to discover relations between genes, environment, and disease. BioData Mining 6, 6.

Swerdlow, S.H., Campo, E., Harris, N.L., Jaffe, E.S., Pileri, S.A., Stein, H., Thiele, J., and Vardiman, J.W. (2008). WHO Classification of Tumours of Haematopoietic and Lymphoid Tissues (Lyon: IARC). 
Taipale, J., Chen, J.K., Cooper, M.K., Wang, B., Mann, R.K., Milenkovic, L., Scott, M.P., and Beachy, P. a (2000). Effects of oncogenic mutations in Smoothened and Patched can be reversed by cyclopamine. Nature 406, 1005-1009.

Takaesu, G., Surabhi, R.M., Park, K.-J., Ninomiya-Tsuji, J., Matsumoto, K., and Gaynor, R.B. (2003). TAK1 is critical for IkappaB kinase-mediated activation of the NF-kappaB pathway. Journal of Molecular Biology 326, 105-115.

Takaesu, G., Inagaki, M., Takubo, K., Mishina, Y., Hess, P.R., Dean, G. a, Yoshimura, A., Matsumoto, K., Suda, T., and Ninomiya-Tsuji, J. (2012). TAK1 (MAP3K7) signaling regulates hematopoietic stem cells through TNF-dependent and -independent mechanisms. PloS One 7, e51073.

Takata, M., and Kurosaki, T. (1996). A role for Bruton's tyrosine kinase in B cell antigen receptormediated activation of phospholipase C-gamma 2. The Journal of Experimental Medicine 184, 31-40.

Tedoldi, S., Paterson, J.C., Cordell, J., Tan, S.-Y., Jones, M., Manek, S., Dei Tos, A.P., Roberton, H., Masir, N., Natkunam, Y., et al. (2006). Jaw1/LRMP, a germinal centre-associated marker for the immunohistological study of B-cell lymphomas. The Journal of Pathology 209, 454-463.

Titsias, M.K., Honkela, A., Lawrence, N.D., and Rattray, M. (2012). Identifying targets of multiple coregulating transcription factors from expression time-series by Bayesian model comparison. BMC Systems Biology 6, 53.

Tosello, V., and Ferrando, A.A. (2013). The NOTCH signaling pathway: role in the pathogenesis of Tcell acute lymphoblastic leukemia and implication for therapy. Therapeutic Advances in Hematology 4, 199-210.

Tweeddale, M.E., Lim, B., Jamal, N., Robinson, J., Zalcberg, J., Lockwood, G., Minden, M.D., and Messner, H.A. (1987). The presence of clonogenic cells in high-grade malignant lymphoma: a prognostic factor. Blood 69, 1307-1314.

Uddin, S., Hussain, A.R., Siraj, A.K., Manogaran, P.S., Al-Jomah, N.A., Moorji, A., Atizado, V., Al-Dayel, F., Belgaumi, A., El-Solh, H., et al. (2006). Role of phosphatidylinositol 3'-kinase/AKT pathway in diffuse large B-cell lymphoma survival. Blood 108, 4178-4186.

Uhmann, A., Dittmann, K., Nitzki, F., Dressel, R., Koleva, M., Frommhold, A., Zibat, A., Binder, C., Adham, I., Nitsche, M., et al. (2007). The Hedgehog receptor Patched controls lymphoid lineage commitment. Blood 110, 1814-1823.

Vallabhapurapu, S., and Karin, M. (2009). Regulation and function of NF-kappaB transcription factors in the immune system. Annual Review of Immunology 27, 693-733.

Vega, M.I., Huerta-Yepez, S., Jazirehi, A.R., Garban, H., and Bonavida, B. (2005). Rituximab (chimeric anti-CD20) sensitizes B-NHL cell lines to Fas-induced apoptosis. Oncogene 24, 8114-8127.

Vlahos, C.J., Matter, W.F., Hui, K.Y., and Brown, R.F. (1994). A specific inhibitor of phosphatidylinositol 3-kinase, 2-(4-morpholinyl)-8-phenyl-4H-1-benzopyran-4-one (LY294002). The Journal of Biological Chemistry 269, 5241-5248.

Vockerodt, M., Haier, B., Buttgereit, P., Tesch, H., and Kube, D. (2001). The Epstein-Barr virus latent membrane protein 1 induces interleukin-10 in Burkitt's lymphoma cells but not in Hodgkin's cells involving the p38/SAPK2 pathway. Virology 280, 183-198. 
Walsh, K., McKinney, M.S., Love, C., Liu, Q., Fan, A., Patel, A., Smith, J., Beaven, A., Jima, D.D., and Dave, S.S. (2013). PAK1 Mediates Resistance to PI3K Inhibition in Lymphomas. Clinical Cancer Research : an Official Journal of the American Association for Cancer Research.

Walther, N., Ulrich, A., Vockerodt, M., von Bonin, F., Klapper, W., Meyer, K., Eberth, S., Pukrop, T., Spang, R., Trümper, L., et al. (2013). Aberrant lymphocyte enhancer-binding factor 1 expression is characteristic for sporadic Burkitt's lymphoma. The American Journal of Pathology 182, 1092-1098.

Wan, J., Sun, L., Mendoza, J.W., Chui, Y.L., Huang, D.P., Chen, Z.J., Suzuki, N., Suzuki, S., Yeh, W.-C., Akira, S., et al. (2004). Elucidation of the c-Jun N-terminal kinase pathway mediated by Estein-Barr virus-encoded latent membrane protein 1. Molecular and Cellular Biology 24, 192-199.

Wang, M., Atayar, C., Rosati, S., Bosga-Bouwer, A., Kluin, P., and Visser, L. (2009). JNK is constitutively active in mantle cell lymphoma: cell cycle deregulation and polyploidy by JNK inhibitor SP600125. The Journal of Pathology 218, 95-103.

Wei, L., and Xu, Z. (2011). Cross-signaling among phosphinositide-3 kinase, mitogen-activated protein kinase and sonic hedgehog pathways exists in esophageal cancer. International Journal of Cancer. Journal International Du Cancer 129, 275-284.

Weston, C.R., and Davis, R.J. (2002). The JNK signal transduction pathway. Current Opinion in Genetics \& Development 12, 14-21.

Wichert, S., Fokianos, K., and Strimmer, K. (2003). Identifying periodically expressed transcripts in microarray time series data. Bioinformatics 20, 5-20.

Wienands, J., Larbolette, O., and Reth, M. (1996). Evidence for a preformed transducer complex organized by the B cell antigen receptor. Proceedings of the National Academy of Sciences of the United States of America 93, 7865-7870.

Willander, K., Dutta, R., Ungerbäck, J., Gunnarsson, R., Juliusson, G., Fredrikson, M., Linderholm, M., and Söderkvist, P. (2013). NOTCH1 mutations influence survival in chronic lymphocytic leukemia patients. BMC Cancer 13, 274.

Wu, L., Chien, W.-M., Hartman, M.E., Moussavi-Harami, F., Liu, Y., and Chin, M.T. (2011). Regulation of MMP10 expression by the transcription factor CHF1/Hey2 is mediated by multiple $\mathrm{E}$ boxes. Biochemical and Biophysical Research Communications 415, 662-668.

Xie, J., Murone, M., Luoh, S.M., Ryan, A., Gu, Q., Zhang, C., Bonifas, J.M., Lam, C.W., Hynes, M., Goddard, A., et al. (1998). Activating Smoothened mutations in sporadic basal-cell carcinoma. Nature $391,90-92$.

Xu, J., Foy, T.M., Laman, J.D., Elliott, E.A., Dunn, J.J., Waldschmidt, T.J., Elsemore, J., Noelle, R.J., and Flavell, R.A. (1994). Mice deficient for the CD40 ligand. Immunity 1, 423-431.

Yamaguchi, K., Shirakabe, K., Shibuya, H., Irie, K., Oishi, I., Ueno, N., Taniguchi, T., Nishida, E., and Matsumoto, K. (1995). Identification of a member of the MAPKKK family as a potential mediator of TGF-beta signal transduction. Science (New York, N.Y.) 270, 2008-2011.

Yamamoto, Y., and Gaynor, R.B. (2004). IKB kinases: key regulators of the NF-KB pathway. Trends in Biochemical Sciences 29, 72-79. 
Yamashita, M., Fatyol, K., Jin, C., Wang, X., Liu, Z., and Zhang, Y.E. (2008). TRAF6 mediates Smadindependent activation of JNK and p38 by TGF-beta. Molecular Cell 31, 918-924.

Yan, L., Mieulet, V., and Lamb, R.F. (2008). mTORC2 is the hydrophobic motif kinase for SGK1. The Biochemical Journal 416, e19-21.

Yang, J., Wang, S., Zhao, G., and Sun, B. (2011). Effect of chemokine receptors CCR7 on disseminated behavior of human T cell lymphoma: clinical and experimental study. Journal of Experimental \& Clinical Cancer Research : CR 30, 51.

Yano, T., van Krieken, J.H., Magrath, I.T., Longo, D.L., Jaffe, E.S., and Raffeld, M. (1992). Histogenetic correlations between subcategories of small noncleaved cell lymphomas. Blood 79, 1282-1290.

Yeung, K.Y., Dombek, K.M., Lo, K., Mittler, J.E., Zhu, J., Schadt, E.E., Bumgarner, R.E., and Raftery, A.E. (2011). Construction of regulatory networks using expression time-series data of a genotyped population. Proceedings of the National Academy of Sciences of the United States of America 108, 19436-19441.

Yilmaz, Z.B., Weih, D.S., Sivakumar, V., and Weih, F. (2003). RelB is required for Peyer's patch development: differential regulation of p52-RelB by lymphotoxin and TNF. The EMBO Journal 22, 121-130.

Yochum, G.S., Cleland, R., and Goodman, R.H. (2008). A genome-wide screen for beta-catenin binding sites identifies a downstream enhancer element that controls c-Myc gene expression. Molecular and Cellular Biology 28, 7368-7379.

Yoon, S., and Seger, R. (2006). The extracellular signal-regulated kinase: multiple substrates regulate diverse cellular functions. Growth Factors (Chur, Switzerland) 24, 21-44.

Yoon, J.W., Gallant, M., Lamm, M.L., Iannaccone, S., Vieux, K.-F., Proytcheva, M., Hyjek, E., lannaccone, P., and Walterhouse, D. (2013). Noncanonical Regulation of the Hedgehog Mediator GLI1 by c-MYC in Burkitt Lymphoma. Molecular Cancer Research : MCR 11, 604-615.

Young, R.M., and Staudt, L.M. (2013). Targeting pathological B cell receptor signalling in lymphoid malignancies. Nature Reviews. Drug Discovery 12, 229-243.

Zheng, B., Fiumara, P., Li, Y. V, Georgakis, G., Snell, V., Younes, M., Vauthey, J.N., Carbone, A., and Younes, A. (2003). MEK/ERK pathway is aberrantly active in Hodgkin disease: a signaling pathway shared by CD30, CD40, and RANK that regulates cell proliferation and survival. Blood 102, 10191027.

Zou, M., and Conzen, S.D. (2005). A new dynamic Bayesian network (DBN) approach for identifying gene regulatory networks from time course microarray data. Bioinformatics (Oxford, England) 21, 71-79.

Zwerner, J.P., Joo, J., Warner, K.L., Christensen, L., Hu-Lieskovan, S., Triche, T.J., and May, W.A. (2008). The EWS/FLI1 oncogenic transcription factor deregulates GLI1. Oncogene 27, 3282-3291. 


\section{Appendix}

Table A-1 Clusters of genes identified upon BCR crosslinked BL2 cells (BHC, SIMoNe). Using Bayesian Hierarchical Clustering sets of genes were built, which show the same gene expression course after anti-IgM treatment of BL cells.

\begin{tabular}{|l|l|l|}
\hline & Probe & Symbol \\
\hline ID & Iuster 4 & \\
\hline $\mathbf{1}$ & 7902398 & SNORD45A \\
\hline $\mathbf{2}$ & 7948908 & SNORD26 \\
\hline $\mathbf{3}$ & 7993664 & CCP110 \\
\hline $\mathbf{4}$ & 8043697 & ANKRD36B \\
\hline $\mathbf{5}$ & 8068522 & TTC3 \\
\hline $\mathbf{6}$ & 8117330 & HIST1H3A \\
\hline $\mathbf{7}$ & 8117580 & HIST1H2AI \\
\hline $\mathbf{8}$ & 8127989 & SNORD50B \\
\hline $\mathbf{9}$ & 8159004 & SNORD24 \\
\hline Cluster 5 & \\
\hline $\mathbf{1}$ & 7900119 & THRAP3 \\
\hline $\mathbf{2}$ & 7904974 & PFN1P2 \\
\hline $\mathbf{3}$ & 7908867 & TMEM183A \\
\hline $\mathbf{4}$ & 7924923 & NUP133 \\
\hline $\mathbf{5}$ & 7957540 & MRPL42 \\
\hline $\mathbf{6}$ & 7959408 & KNTC1 \\
\hline $\mathbf{7}$ & 7969428 & UCHL3 \\
\hline $\mathbf{8}$ & 7969703 & IPO5 \\
\hline $\mathbf{9}$ & 7981335 & HSP90AA1 \\
\hline $\mathbf{1 0}$ & 7985829 & FANCI \\
\hline $\mathbf{1 1}$ & 8014650 & NPEPPS \\
\hline $\mathbf{1 2}$ & 8050565 & PUM2 \\
\hline $\mathbf{1 3}$ & 8059319 & FARSB \\
\hline $\mathbf{1 4}$ & 8074925 & GUSBP11 \\
\hline $\mathbf{1 5}$ & 8085374 & RAF1 \\
\hline $\mathbf{1 6}$ & 8087874 & WDR82 \\
\hline $\mathbf{1 7}$ & 8091452 & TMEM183B \\
\hline $\mathbf{1 8}$ & 8099926 & PDS5A \\
\hline $\mathbf{1 9}$ & 8105523 & KIF2A \\
\hline $\mathbf{2 0}$ & 8111455 & GUSBP1 \\
\hline $\mathbf{2 1}$ & 8112558 & GUSBP2 \\
\hline $\mathbf{2 2}$ & 8138670 & HNRNPA2B1 \\
\hline $\mathbf{2 3}$ & 8162313 & IARS \\
\hline $\mathbf{2 4}$ & 8162514 & FANCC \\
\hline Cluster 6 & \\
\hline $\mathbf{1}$ & 7897648 & PEX14 \\
\hline $\mathbf{2}$ & 7897849 & MIIP \\
\hline $\mathbf{3}$ & 7899134 & CEP85 \\
\hline $\mathbf{4}$ & 7899244 & PIGV \\
\hline $\mathbf{5}$ & 7900699 & CDC20 \\
\hline $\mathbf{6}$ & 7904812 & PIAS3 \\
\hline $\mathbf{7}$ & 7905198 & TARS2 \\
\hline $\mathbf{8}$ & 7907092 & MPZL1 \\
\hline & & \\
\hline
\end{tabular}

\begin{tabular}{|l|l|l|}
\hline $\mathbf{9}$ & 7909529 & RCOR3 \\
\hline $\mathbf{1 0}$ & 7909586 & PPP2R5A \\
\hline $\mathbf{1 1}$ & 7909898 & MIA3 \\
\hline $\mathbf{1 2}$ & 7911591 & SLC35E2B \\
\hline $\mathbf{1 3}$ & 7911600 & NADK \\
\hline $\mathbf{1 4}$ & 7911862 & LRRC47 \\
\hline $\mathbf{1 5}$ & 7919825 & ARNT \\
\hline $\mathbf{1 6}$ & 7921806 & B4GALT3 \\
\hline $\mathbf{1 7}$ & 7934477 & CAMK2G \\
\hline $\mathbf{1 8}$ & 7941769 & KDM2A \\
\hline $\mathbf{1 9}$ & 7941879 & TBC1D10C \\
\hline $\mathbf{2 0}$ & 7941927 & AIP \\
\hline $\mathbf{2 1}$ & 7945357 & SIRT3 \\
\hline $\mathbf{2 2}$ & 7948685 & TUT1 \\
\hline $\mathbf{2 3}$ & 7949518 & FIBP \\
\hline $\mathbf{2 4}$ & 7949674 & RBM4B \\
\hline $\mathbf{2 5}$ & 7951752 & USP28 \\
\hline $\mathbf{2 6}$ & 7952484 & TMEM218 \\
\hline $\mathbf{2 7}$ & 7952988 & ERC1 \\
\hline $\mathbf{2 8}$ & 7955217 & SPATS2 \\
\hline $\mathbf{2 9}$ & 7959777 & BRI3BP \\
\hline $\mathbf{3 0}$ & 7960702 & CDCA3 \\
\hline $\mathbf{3 1}$ & 7961339 & LRP6 \\
\hline $\mathbf{3 2}$ & 7963851 & KIAA0748 \\
\hline $\mathbf{3 3}$ & 7964203 & BAZ2A \\
\hline $\mathbf{3 4}$ & 7969935 & ERCC5 \\
\hline $\mathbf{3 5}$ & 7971039 & FAM48A \\
\hline $\mathbf{3 6}$ & 7973732 & KHNYN \\
\hline $\mathbf{3 7}$ & 7979849 & DCAF5 \\
\hline $\mathbf{3 8}$ & 7986186 & RCCD1 \\
\hline $\mathbf{3 9}$ & 7987405 & RASGRP1 \\
\hline $\mathbf{4 0}$ & 7989759 & PARP16 \\
\hline $\mathbf{4 1}$ & 7990757 & CTSH \\
\hline $\mathbf{4 2}$ & 7995492 & ADCY7 \\
\hline $\mathbf{4 3}$ & 7996041 & COQ9 \\
\hline $\mathbf{4 4}$ & 7996744 & NFATC3 \\
\hline $\mathbf{4 5}$ & 7999304 & FAM86A \\
\hline $\mathbf{4 6}$ & 7999596 & KIAA2013 \\
\hline $\mathbf{4 7}$ & 8000263 & COG7 \\
\hline $\mathbf{4 8}$ & 8000706 & CDIPT \\
\hline $\mathbf{4 9}$ & 8002381 & COG4 \\
\hline $\mathbf{5 0}$ & 8002904 & ADAT1 \\
\hline $\mathbf{5 1}$ & 8003922 & MED11 \\
\hline $\mathbf{5 2}$ & 8005839 & TMEM97 \\
\hline $\mathbf{5 3}$ & 8009164 & DCAF7 \\
\hline $\mathbf{5 4}$ & 8014551 & SYNRG \\
\hline $\mathbf{5 5}$ & 8014723 & PIP4K2B \\
\hline & & \\
\hline
\end{tabular}




\begin{tabular}{|c|c|c|}
\hline 56 & 8016847 & TRIM25 \\
\hline 57 & 8017019 & MTMR4 \\
\hline 58 & 8018694 & PRPSAP1 \\
\hline 59 & 8025053 & TNFSF9 \\
\hline 60 & 8026982 & MPV17L2 \\
\hline 61 & 8029856 & ARHGAP35 \\
\hline 62 & 8032455 & PLEKHJ1 \\
\hline 63 & 8034122 & SPC24 \\
\hline 64 & 8034589 & FARSA \\
\hline 65 & 8035249 & NR2F6 \\
\hline 66 & 8036483 & YIF1B \\
\hline 67 & 8038117 & DBP \\
\hline 68 & 8047288 & SGOL2 \\
\hline 69 & 8048175 & SMARCAL1 \\
\hline 70 & 8051012 & PREB \\
\hline 71 & 8051298 & GALNT14 \\
\hline 72 & 8060705 & MAVS \\
\hline 73 & 8065637 & COMMD7 \\
\hline 74 & 8065992 & NFS1 \\
\hline 75 & 8066964 & TMEM189 \\
\hline 76 & 8072529 & DEPDC5 \\
\hline 77 & 8078450 & CRTAP \\
\hline 78 & 8080781 & PXK \\
\hline 79 & 8087308 & USP19 \\
\hline 80 & 8089040 & MINA \\
\hline 81 & 8093976 & TBC1D14 \\
\hline 82 & 8095574 & DCK \\
\hline 83 & 8095751 & PARM1 \\
\hline 84 & 8098581 & SNX25 \\
\hline 85 & 8118116 & MICB \\
\hline 86 & 8118863 & ANKS1A \\
\hline 87 & 8124211 & GPLD1 \\
\hline 88 & 8125470 & HLA-DOB \\
\hline 89 & 8131957 & SNX10 \\
\hline 90 & 8140107 & DNAJC30 \\
\hline 91 & 8143070 & WDR91 \\
\hline 92 & 8143863 & FASTK \\
\hline 93 & 8145418 & CDCA2 \\
\hline 94 & 8149389 & FAM86B1 \\
\hline 95 & 8155327 & ALDH1B1 \\
\hline 96 & 8164304 & ST6GALNAC6 \\
\hline 97 & 8164481 & C9orf114 \\
\hline 98 & 8165064 & UBAC1 \\
\hline 99 & 8176245 & F8A1 \\
\hline 100 & 8177955 & MICB \\
\hline 101 & 8178833 & HLA-DOB \\
\hline 102 & 8180339 & ST6GALNAC6 \\
\hline \multicolumn{3}{|c|}{ Cluster 7} \\
\hline 1 & 7908147 & TSEN15 \\
\hline 2 & 7911897 & C1orf174 \\
\hline 3 & 7942626 & UVRAG \\
\hline 4 & 7947784 & ARFGAP2 \\
\hline 5 & 7956401 & SHMT2 \\
\hline
\end{tabular}

\begin{tabular}{|c|c|c|}
\hline 6 & 7968199 & CDK8 \\
\hline 7 & 7991406 & PRC1 \\
\hline 8 & 8002729 & GLG1 \\
\hline 9 & 8021727 & CNDP2 \\
\hline 10 & 8027139 & RFXANK \\
\hline 11 & 8032525 & SLC39A3 \\
\hline 12 & 8051998 & MCFD2 \\
\hline 13 & 8064156 & ZGPAT \\
\hline 14 & 8069933 & MIS18A \\
\hline 15 & 8073379 & L3MBTL2 \\
\hline 16 & 8080645 & APPL1 \\
\hline 17 & 8093112 & UBXN7 \\
\hline 18 & 8093462 & MAEA \\
\hline 19 & 8096688 & GSTCD \\
\hline 20 & 8108861 & NDFIP1 \\
\hline 21 & 8110589 & CNOT6 \\
\hline 22 & 8111974 & PAIP1 \\
\hline 23 & 8132539 & DBNL \\
\hline 24 & 8139356 & NUDCD3 \\
\hline 25 & 8143387 & MKRN1 \\
\hline 26 & 8145586 & ELP3 \\
\hline 27 & 8156897 & C9orf30 \\
\hline 28 & 8160953 & PIGO \\
\hline 29 & 8164013 & STRBP \\
\hline 30 & 8180394 & POLR2M \\
\hline \multicolumn{3}{|c|}{ Cluster 8} \\
\hline 1 & 7899604 & $\mathrm{ZCCHC} 17$ \\
\hline 2 & 7901479 & ZYG11B \\
\hline 3 & 7902308 & FPGT \\
\hline 4 & 7906652 & NIT1 \\
\hline 5 & 7907353 & METTL13 \\
\hline 6 & 7912292 & LZIC \\
\hline 7 & 7914809 & KIAA0319L \\
\hline 8 & 7915775 & IPP \\
\hline 9 & 7915846 & MKNK1 \\
\hline 10 & 7921738 & USF1 \\
\hline 11 & 7935251 & TCTN3 \\
\hline 12 & 7935647 & COX15 \\
\hline 13 & 7939087 & C11orf46 \\
\hline 14 & 7946089 & TRIM5 \\
\hline 15 & 7948249 & SLC43A1 \\
\hline 16 & 7952914 & CCDC77 \\
\hline 17 & 7955450 & LETMD1 \\
\hline 18 & 7958275 & POLR3B \\
\hline 19 & 7969374 & BORA \\
\hline 20 & 7973036 & PARP2 \\
\hline 21 & 7974621 & ARID4A \\
\hline 22 & 7980403 & C14orf133 \\
\hline 23 & 7982154 & HERC2P2 \\
\hline 24 & 7982723 & IVD \\
\hline 25 & 7986569 & HERC2P2 \\
\hline 26 & 7986701 & HERC2P2 \\
\hline 27 & 7989834 & C15orf44 \\
\hline
\end{tabular}




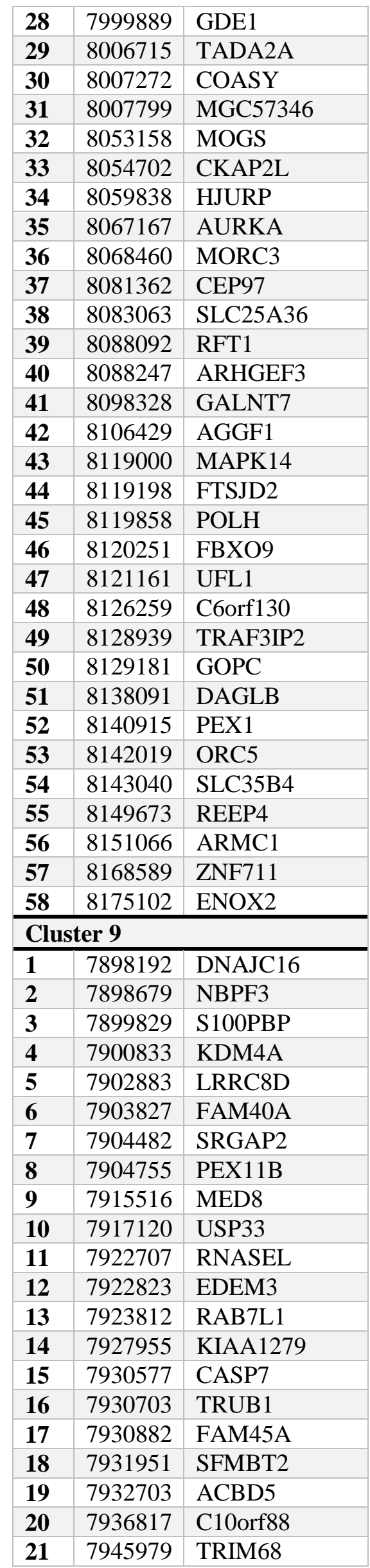

\begin{tabular}{|c|c|c|}
\hline 22 & 7951422 & KIAA1826 \\
\hline 23 & 7954969 & IRAK4 \\
\hline 24 & 7959563 & C12orf65 \\
\hline 25 & 7960261 & RAD52 \\
\hline 26 & 7963935 & DNAJC14 \\
\hline 27 & 7967240 & VPS33A \\
\hline 28 & 7969228 & ALG11 \\
\hline 29 & 7976307 & GOLGA5 \\
\hline 30 & 7985053 & FBXO22 \\
\hline 31 & 7985080 & ISL2 \\
\hline 32 & 7988838 & LEO1 \\
\hline 33 & 7991357 & AP3S2 \\
\hline 34 & 7995362 & GPT2 \\
\hline 35 & 7999478 & TXNDC11 \\
\hline 36 & 8000236 & CDR2 \\
\hline 37 & 8009552 & C17orf80 \\
\hline 38 & 8014264 & PEX12 \\
\hline 39 & 8016562 & SPOP \\
\hline 40 & 8017555 & ERN1 \\
\hline 41 & 8021275 & POLI \\
\hline 42 & 8021716 & TIMM21 \\
\hline 43 & 8034565 & DNASE2 \\
\hline 44 & 8042310 & SLC1A4 \\
\hline 45 & 8047443 & STRADB \\
\hline 46 & 8050443 & SMC6 \\
\hline 47 & 8062545 & ACTR5 \\
\hline 48 & 8072108 & ASPHD2 \\
\hline 49 & 8072153 & CCDC117 \\
\hline 50 & 8073513 & CCDC134 \\
\hline 51 & 8080926 & ARL6IP5 \\
\hline 52 & 8081612 & ABHD10 \\
\hline 53 & 8081667 & SLC35A5 \\
\hline 54 & 8081953 & GTF2E1 \\
\hline 55 & 8083282 & HPS3 \\
\hline 56 & 8084360 & ABCF3 \\
\hline 57 & 8085852 & NGLY1 \\
\hline 58 & 8090420 & TPRA1 \\
\hline 59 & 8090577 & MBD4 \\
\hline 60 & 8091737 & IFT80 \\
\hline 61 & 8093936 & MRFAP1 \\
\hline 62 & 8095262 & REST \\
\hline 63 & 8102745 & PGRMC2 \\
\hline 64 & 8108472 & PURA \\
\hline 65 & 8109576 & THG1L \\
\hline 66 & 8111960 & C5orf34 \\
\hline 67 & 8113733 & CEP120 \\
\hline 68 & 8113790 & MARCH3 \\
\hline 69 & 8121886 & HINT3 \\
\hline 70 & 8124280 & FAM65B \\
\hline 71 & 8127787 & IBTK \\
\hline 72 & 8132318 & ANLN \\
\hline 73 & 8133114 & VKORC1L1 \\
\hline 74 & 8133809 & RSBN1L \\
\hline
\end{tabular}




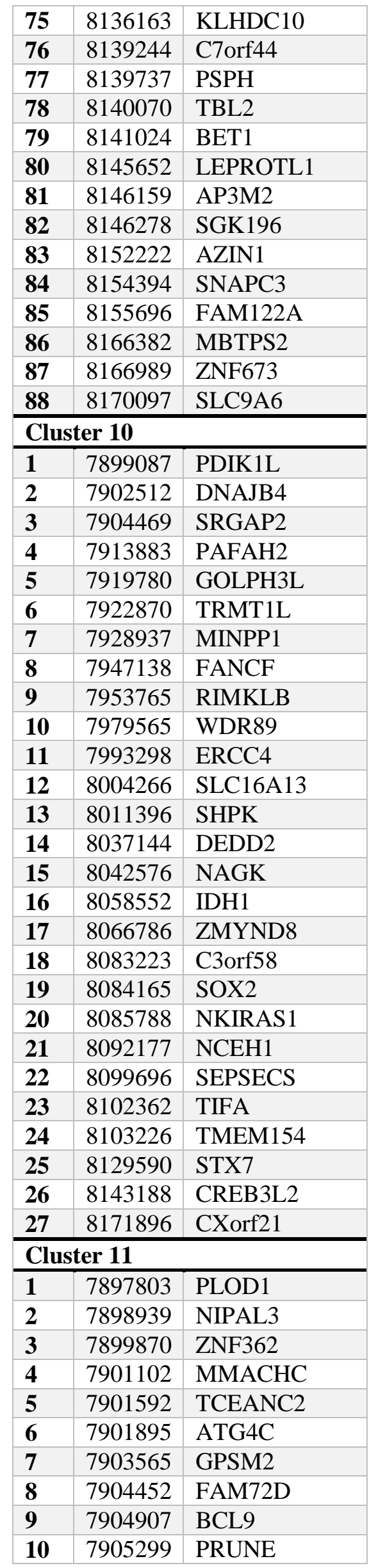

\begin{tabular}{|c|c|c|}
\hline 11 & 7906671 & USP21 \\
\hline 12 & 7907058 & POU2F1 \\
\hline 13 & 7907079 & RCSD1 \\
\hline 14 & 7909146 & FAM72D \\
\hline 15 & 7913682 & HMGCL \\
\hline 16 & 7913694 & FUCA1 \\
\hline 17 & 7914917 & LSM10 \\
\hline 18 & 7915472 & SLC2A1 \\
\hline 19 & 7915787 & PIK3R3 \\
\hline 20 & 7919591 & FAM72D \\
\hline 21 & 7923662 & PIK3C2B \\
\hline 22 & 7923792 & SLC45A3 \\
\hline 23 & 7925773 & ZNF692 \\
\hline 24 & 7928491 & KAT6B \\
\hline 25 & 7929322 & CYP26A1 \\
\hline 26 & 7933228 & MARCH8 \\
\hline 27 & 7938183 & ZNF215 \\
\hline 28 & 7941104 & ARL2 \\
\hline 29 & 7942562 & NEU3 \\
\hline 30 & 7944152 & IL10RA \\
\hline 31 & 7945132 & FLI1 \\
\hline 32 & 7945875 & FAM86B1 \\
\hline 33 & 7952601 & ETS1 \\
\hline 34 & 7952707 & PRDM10 \\
\hline 35 & 7954717 & BICD1 \\
\hline 36 & 7955195 & TROAP \\
\hline 37 & 7957167 & TMEM19 \\
\hline 38 & 7957759 & APAF1 \\
\hline 39 & 7958439 & USP30 \\
\hline 40 & 7959298 & TMEM120B \\
\hline 41 & 7959354 & BCL7A \\
\hline 42 & 7960910 & AICDA \\
\hline 43 & 7961983 & TM7SF3 \\
\hline 44 & 7965565 & USP44 \\
\hline 45 & 7968789 & RGCC \\
\hline 46 & 7971486 & KIAA0226L \\
\hline 47 & 7974697 & DAAM1 \\
\hline 48 & 7975311 & EXD2 \\
\hline 49 & 7975361 & KIAA0247 \\
\hline 50 & 7975787 & JDP2 \\
\hline 51 & 7977868 & C14orf93 \\
\hline 52 & 7986463 & LRRK1 \\
\hline 53 & 7988426 & SLC30A4 \\
\hline 54 & 7988970 & FAM214A \\
\hline 55 & 7994655 & C16orf53 \\
\hline 56 & 7997735 & FOXL1 \\
\hline 57 & 8001178 & C16orf87 \\
\hline 58 & 8006345 & RHOT1 \\
\hline 59 & 8007471 & NBR1 \\
\hline 60 & 8008339 & RSAD1 \\
\hline 61 & 8010139 & SEC14L1 \\
\hline 62 & 8011415 & P2RX5 \\
\hline 63 & 8013804 & DHRS13 \\
\hline
\end{tabular}




\begin{tabular}{|l|l|l|}
\hline $\mathbf{6 4}$ & 8015655 & FAM134C \\
\hline $\mathbf{6 5}$ & 8019018 & CBX4 \\
\hline $\mathbf{6 6}$ & 8021565 & PHLPP1 \\
\hline $\mathbf{6 7}$ & 8026712 & ANKLE1 \\
\hline $\mathbf{6 8}$ & 8029465 & BCL3 \\
\hline $\mathbf{6 9}$ & 8031522 & ZNF581 \\
\hline $\mathbf{7 0}$ & 8037186 & LIPE \\
\hline $\mathbf{7 1}$ & 8037495 & ZNF296 \\
\hline $\mathbf{7 2}$ & 8039928 & FAM72D \\
\hline $\mathbf{7 3}$ & 8040365 & TRIB2 \\
\hline $\mathbf{7 4}$ & 8048898 & SP140 \\
\hline $\mathbf{7 5}$ & 8052669 & SERTAD2 \\
\hline $\mathbf{7 6}$ & 8056766 & SLC25A12 \\
\hline $\mathbf{7 7}$ & 8058161 & ORC2 \\
\hline $\mathbf{7 8}$ & 8058927 & TMBIM1 \\
\hline $\mathbf{7 9}$ & 8060977 & C20orf94 \\
\hline $\mathbf{8 0}$ & 8085233 & RPUSD3 \\
\hline $\mathbf{8 1}$ & 8087852 & TLR9 \\
\hline $\mathbf{8 2}$ & 8089261 & CBLB \\
\hline $\mathbf{8 3}$ & 8092691 & BCL6 \\
\hline $\mathbf{8 4}$ & 8096070 & BMP3 \\
\hline $\mathbf{8 5}$ & 8097417 & PHF17 \\
\hline $\mathbf{8 6}$ & 8097867 & KIAA0922 \\
\hline $\mathbf{8 7}$ & 8098423 & NEIL3 \\
\hline $\mathbf{8 8}$ & 8102342 & ELOVL6 \\
\hline $\mathbf{8 9}$ & 8104901 & IL7R \\
\hline $\mathbf{9 0}$ & 8105878 & RAD17 \\
\hline $\mathbf{9 1}$ & 8106107 & PTCD2 \\
\hline $\mathbf{9 2}$ & 8108301 & KIF20A \\
\hline $\mathbf{9 3}$ & 8108447 & CXXC5 \\
\hline $\mathbf{9 4}$ & 8111136 & FAM134B \\
\hline $\mathbf{9 5}$ & 8111552 & NADKD1 \\
\hline $\mathbf{9 6}$ & 8117120 & ID4 \\
\hline $\mathbf{9 7}$ & 8117165 & SOX4 \\
\hline $\mathbf{9 8}$ & 8117435 & BTN3A2 \\
\hline $\mathbf{9 9}$ & 8122013 & L3MBTL3 \\
\hline $\mathbf{1 0 0}$ & 8126018 & STK38 \\
\hline $\mathbf{1 0 1}$ & 8126428 & TRERF1 \\
\hline $\mathbf{1 0 2}$ & 8136341 & BPGM \\
\hline $\mathbf{1 0 3}$ & 8138030 & PMS2 \\
\hline $\mathbf{1 0 4}$ & 8138088 & C7orf70 \\
\hline $\mathbf{1 0 5}$ & 8142096 & ATXN7L1 \\
\hline $\mathbf{1 0 6}$ & 8148158 & WDR67 \\
\hline $\mathbf{1 0 7}$ & 8148317 & MYC \\
\hline $\mathbf{1 0 8}$ & 8149387 & FAM86B1 \\
\hline $\mathbf{1 0 9}$ & 8151074 & PDE7A \\
\hline $\mathbf{1 1 0}$ & 8153935 & ZNF252 \\
\hline $\mathbf{1 1 1}$ & 8155332 & C7orf70 \\
\hline $\mathbf{1 1 3}$ & 8160835 & C9orf23 \\
\hline $\mathbf{1 1 4}$ & 8162438 & BICD2 \\
\hline & 8164252 & SH2D3C \\
\hline
\end{tabular}

\begin{tabular}{|c|c|c|}
\hline 117 & 8171029 & F8A1 \\
\hline 118 & 8171136 & P2RY8 \\
\hline 119 & 8172088 & BCOR \\
\hline 120 & 8173208 & SPIN4 \\
\hline 121 & 8173232 & FAM123B \\
\hline 122 & 8177026 & P2RY8 \\
\hline 123 & 8177478 & RAD17 \\
\hline 124 & 8180336 & SLC35E2 \\
\hline 125 & 8180412 & ST6GALNAC4 \\
\hline 126 & 8180413 & ST6GALNAC4 \\
\hline \multicolumn{3}{|c|}{$\begin{array}{l}\text { Cluster } 12 \\
\end{array}$} \\
\hline 1 & 7897482 & PIK3CD \\
\hline 2 & 7898677 & HS6ST1 \\
\hline 3 & 7901140 & MAST2 \\
\hline 4 & 7903719 & AMPD2 \\
\hline 5 & 7905831 & FLAD1 \\
\hline 6 & 7906061 & SYT11 \\
\hline 7 & 7906079 & RAB25 \\
\hline 8 & 7906767 & FCGR2C \\
\hline 9 & 7912224 & SLC2A5 \\
\hline 10 & 7915567 & ERI3 \\
\hline 11 & 7916403 & SSBP3 \\
\hline 12 & 7920877 & ARHGEF2 \\
\hline 13 & 7920912 & UBQLN4 \\
\hline 14 & 7923824 & SLC41A1 \\
\hline 15 & 7925978 & FAM208B \\
\hline 16 & 7926037 & PFKFB3 \\
\hline 17 & 7926170 & DHTKD1 \\
\hline 18 & 7927425 & WDFY4 \\
\hline 19 & 7929990 & PPRC1 \\
\hline 20 & 7931930 & PRKCQ \\
\hline 21 & 7933760 & CCDC6 \\
\hline 22 & 7935968 & LDB1 \\
\hline 23 & 7937485 & PNPLA2 \\
\hline 24 & 7937892 & PGAP2 \\
\hline 25 & 7940349 & CCDC86 \\
\hline 26 & 7941302 & FAM89B \\
\hline 27 & 7941457 & CCDC85B \\
\hline 28 & 7941694 & RBM14 \\
\hline 29 & 7941743 & LRFN4 \\
\hline 30 & 7941797 & ADRBK1 \\
\hline 31 & 7942342 & INPPL1 \\
\hline 32 & 7944335 & CXCR5 \\
\hline 33 & 7945539 & SLC25A22 \\
\hline 34 & 7948565 & CYBASC3 \\
\hline 35 & 7949146 & SF1 \\
\hline 36 & 7949172 & MAP4K2 \\
\hline 37 & 7949206 & MEN1 \\
\hline 38 & 7949264 & EHD1 \\
\hline 39 & 7949383 & SYVN1 \\
\hline 40 & 7949746 & POLD4 \\
\hline 41 & 7949904 & UNC93B1 \\
\hline 42 & 7950086 & NUMA1 \\
\hline
\end{tabular}




\begin{tabular}{|c|c|c|}
\hline 43 & 7950248 & FCHSD2 \\
\hline 44 & 7951873 & SIK3 \\
\hline 45 & 7952132 & SLC37A4 \\
\hline 46 & 7952830 & NCAPD3 \\
\hline 47 & 7953651 & PEX5 \\
\hline 48 & 7953981 & ETV6 \\
\hline 49 & 7954104 & ATF7IP \\
\hline 50 & 7955019 & ARID2 \\
\hline 51 & 7956220 & OBFC2B \\
\hline 52 & 7957126 & KCNMB4 \\
\hline 53 & 7958950 & C12orf52 \\
\hline 54 & 7958960 & TPCN1 \\
\hline 55 & 7959025 & RNFT2 \\
\hline 56 & 7962659 & HDAC7 \\
\hline 57 & 7963988 & SMARCC2 \\
\hline 58 & 7964413 & R3HDM2 \\
\hline 59 & 7966542 & RASAL1 \\
\hline 60 & 7967358 & PITPNM2 \\
\hline 61 & 7967588 & DHX37 \\
\hline 62 & 7967789 & PXMP2 \\
\hline 63 & 7968126 & LSP1 \\
\hline 64 & 7969533 & SLAIN1 \\
\hline 65 & 7971015 & SMAD9 \\
\hline 66 & 7973709 & NFATC4 \\
\hline 67 & 7977319 & PLD4 \\
\hline 68 & 7977344 & PACS2 \\
\hline 69 & 7980327 & ANGEL1 \\
\hline 70 & 7980970 & ITPK1 \\
\hline 71 & 7981538 & JAG2 \\
\hline 72 & 7982326 & KLF13 \\
\hline 73 & 7982620 & EIF2AK4 \\
\hline 74 & 7987675 & RPAP1 \\
\hline 75 & 7990033 & TLE3 \\
\hline 76 & 7990487 & SIN3A \\
\hline 77 & 7992402 & NDUFB10 \\
\hline 78 & 7992474 & TSC2 \\
\hline 79 & 7992775 & PAQR4 \\
\hline 80 & 7993478 & $\mathrm{ABCC} 1$ \\
\hline 81 & 7994109 & PLK1 \\
\hline 82 & 7994161 & RBBP6 \\
\hline 83 & 7995976 & CPNE2 \\
\hline 84 & 7996608 & RLTPR \\
\hline 85 & 7996785 & PRMT7 \\
\hline 86 & 7997427 & CMIP \\
\hline 87 & 7997680 & KIAA0182 \\
\hline 88 & 7998774 & RNPS1 \\
\hline 89 & 7999102 & TFAP4 \\
\hline 90 & 7999173 & FAM100A \\
\hline 91 & 8004804 & PFAS \\
\hline 92 & 8005978 & TRAF4 \\
\hline 93 & 8006063 & ANKRD13B \\
\hline 94 & 8006298 & RAB11FIP4 \\
\hline 95 & 8006788 & MLLT6 \\
\hline
\end{tabular}

\begin{tabular}{|c|c|c|}
\hline 96 & 8007302 & TUBG1 \\
\hline 97 & 8010673 & SLC25A10 \\
\hline 98 & 8011093 & SLC43A2 \\
\hline 99 & 8011262 & MNT \\
\hline 100 & 8011516 & ATP2A3 \\
\hline 101 & 8011747 & SLC25A11 \\
\hline 102 & 8012304 & TRAPPC1 \\
\hline 103 & 8012376 & VAMP2 \\
\hline 104 & 8012958 & UBE2S \\
\hline 105 & 8013179 & ATPAF2 \\
\hline 106 & 8014700 & C17orf96 \\
\hline 107 & 8014706 & PCGF2 \\
\hline 108 & 8015412 & JUP \\
\hline 109 & 8015526 & KAT2A \\
\hline 110 & 8016546 & ZNF652 \\
\hline 111 & 8017511 & CD79B \\
\hline 112 & 8018708 & UBE2O \\
\hline 113 & 8019280 & PCYT2 \\
\hline 114 & 8019357 & DCXR \\
\hline 115 & 8019778 & PCYT2 \\
\hline 116 & 8024111 & CNN2 \\
\hline 117 & 8024420 & SF3A2 \\
\hline 118 & 8024485 & GADD45B \\
\hline 119 & 8024798 & CCDC94 \\
\hline 120 & 8025142 & ARHGEF18 \\
\hline 121 & 8025382 & CERS4 \\
\hline 122 & 8025601 & ICAM1 \\
\hline 123 & 8026047 & JUNB \\
\hline 124 & 8026533 & HSH2D \\
\hline 125 & 8026579 & TMEM38A \\
\hline 126 & 8026830 & FCHO1 \\
\hline 127 & 8028286 & SIPA1L3 \\
\hline 128 & 8028397 & FAM98C \\
\hline 129 & 8028930 & RAB4B \\
\hline 130 & 8029521 & TOMM40 \\
\hline 131 & 8030525 & PTOV1 \\
\hline 132 & 8030671 & SPIB \\
\hline 133 & 8031526 & CCDC106 \\
\hline 134 & 8031913 & TRIM28 \\
\hline 135 & 8032076 & RNF126 \\
\hline 136 & 8032365 & KLF16 \\
\hline 137 & 8032380 & BTBD2 \\
\hline 138 & 8032392 & MKNK2 \\
\hline 139 & 8032530 & SGTA \\
\hline 140 & 8032755 & ZBTB7A \\
\hline 141 & 8032899 & TICAM1 \\
\hline 142 & 8033190 & SLC25A23 \\
\hline 143 & 8033362 & INSR \\
\hline 144 & 8034130 & KANK2 \\
\hline 145 & 8034454 & DHPS \\
\hline 146 & 8034754 & SAMD1 \\
\hline 147 & 8034783 & LPHN1 \\
\hline 148 & 8035600 & TMEM161A \\
\hline
\end{tabular}




\begin{tabular}{|c|c|c|}
\hline 149 & 8038261 & GYS1 \\
\hline 150 & 8039306 & RDH13 \\
\hline 151 & 8039316 & PPP1R12C \\
\hline 152 & 8039413 & PPP6R1 \\
\hline 153 & 8039491 & UBE2S \\
\hline 154 & 8040238 & HPCAL1 \\
\hline 155 & 8041617 & MTA3 \\
\hline 156 & 8041763 & PRKCE \\
\hline 157 & 8041826 & TTC7A \\
\hline 158 & 8045171 & IMP4 \\
\hline 159 & 8045182 & PTPN18 \\
\hline 160 & 8046815 & ZNF804A \\
\hline 161 & 8047565 & FAM117B \\
\hline 162 & 8048257 & PNKD \\
\hline 163 & 8048452 & WNT10A \\
\hline 164 & 8049246 & INPP5D \\
\hline 165 & 8049317 & DGKD \\
\hline 166 & 8049689 & RNPEPL1 \\
\hline 167 & 8051814 & ZFP36L2 \\
\hline 168 & 8052399 & BCL11A \\
\hline 169 & 8053009 & FBXO41 \\
\hline 170 & 8055130 & HS6ST1 \\
\hline 171 & 8055208 & CCDC115 \\
\hline 172 & 8058973 & ZNF142 \\
\hline 173 & 8059650 & SP110 \\
\hline 174 & 8060353 & RBCK1 \\
\hline 175 & 8060675 & CDC25B \\
\hline 176 & 8064100 & PPDPF \\
\hline 177 & 8064790 & RASSF2 \\
\hline 178 & 8065683 & SNTA1 \\
\hline 179 & 8065693 & NECAB3 \\
\hline 180 & 8066939 & B4GALT5 \\
\hline 181 & 8070503 & PRDM15 \\
\hline 182 & 8070557 & ZNF295 \\
\hline 183 & 8070912 & SLC19A1 \\
\hline 184 & 8071339 & ZDHHC8 \\
\hline 185 & 8071566 & PPIL2 \\
\hline 186 & 8071691 & BCR \\
\hline 187 & 8072757 & CSF2RB \\
\hline 188 & 8074003 & TRABD \\
\hline 189 & 8075430 & MORC2 \\
\hline 190 & 8075493 & PATZ1 \\
\hline 191 & 8075785 & FOXRED2 \\
\hline 192 & 8075910 & RAC2 \\
\hline 193 & 8076056 & CSNK1E \\
\hline 194 & 8076690 & KIAA0930 \\
\hline 195 & 8077042 & SBF1 \\
\hline 196 & 8078729 & MYD88 \\
\hline 197 & 8079037 & TRAK1 \\
\hline 198 & 8079462 & NBEAL2 \\
\hline 199 & 8079796 & RNF123 \\
\hline 200 & 8080093 & RBM15B \\
\hline 201 & 8080487 & PRKCD \\
\hline
\end{tabular}

\begin{tabular}{|c|c|c|}
\hline 202 & 8081465 & BBX \\
\hline 203 & 8082368 & PODXL2 \\
\hline 204 & 8084100 & USP13 \\
\hline 205 & 8086125 & TRANK1 \\
\hline 206 & 8087935 & NT5DC2 \\
\hline 207 & 8090433 & MGLL \\
\hline 208 & 8093219 & BDH1 \\
\hline 209 & 8093440 & FGFRL1 \\
\hline 210 & 8093916 & MAN2B2 \\
\hline 211 & 8094240 & CD38 \\
\hline 212 & 8099965 & ACOT7 \\
\hline 213 & 8102232 & LEF1 \\
\hline 214 & 8108321 & FAM53C \\
\hline 215 & 8109149 & PCYOX1L \\
\hline 216 & 8112107 & PPAP2A \\
\hline 217 & 8112940 & SSBP2 \\
\hline 218 & 8114215 & PITX1 \\
\hline 219 & 8114511 & MZB1 \\
\hline 220 & 8115543 & EBF1 \\
\hline 221 & 8115927 & RNF44 \\
\hline 222 & 8117243 & LRRC16A \\
\hline 223 & 8119016 & MAPK13 \\
\hline 224 & 8119582 & KIAA0240 \\
\hline 225 & 8121076 & PNRC1 \\
\hline 226 & 8122971 & ARID1B \\
\hline 227 & 8124813 & MDC1 \\
\hline 228 & 8125172 & EHMT2 \\
\hline 229 & 8126371 & CCND3 \\
\hline 230 & 8128818 & WASF1 \\
\hline 231 & 8130185 & ZBTB2 \\
\hline 232 & 8131091 & MAFK \\
\hline 233 & 8133233 & AUTS2 \\
\hline 234 & 8133331 & POM121 \\
\hline 235 & 8135114 & CUX1 \\
\hline 236 & 8135149 & $\mathrm{SH} 2 \mathrm{~B} 2$ \\
\hline 237 & 8137225 & REPIN1 \\
\hline 238 & 8138504 & RAPGEF5 \\
\hline 239 & 8139057 & ELMO1 \\
\hline 240 & 8139270 & RASA4 \\
\hline 241 & 8140398 & YWHAG \\
\hline 242 & 8141768 & RASA4 \\
\hline 243 & 8141803 & RASA4 \\
\hline 244 & 8142981 & PODXL \\
\hline 245 & 8143327 & PARP12 \\
\hline 246 & 8145977 & PLEKHA2 \\
\hline 247 & 8147040 & ZBTB10 \\
\hline 248 & 8148694 & GRINA \\
\hline 249 & 8149612 & LZTS1 \\
\hline 250 & 8149979 & C8orf80 \\
\hline 251 & 8152133 & RRM2B \\
\hline 252 & 8153497 & SCRIB \\
\hline 253 & 8153790 & TONSL \\
\hline 254 & 8155707 & TJP2 \\
\hline
\end{tabular}




\begin{tabular}{|c|c|c|}
\hline 255 & 8156452 & FAM120A \\
\hline 256 & 8158829 & PRRC2B \\
\hline 257 & 8158839 & PRRC2B \\
\hline 258 & 8159249 & MRPS2 \\
\hline 259 & 8160682 & NOL6 \\
\hline 260 & 8162533 & PTCH1 \\
\hline 261 & 8162729 & TRIM14 \\
\hline 262 & 8162803 & ANKS6 \\
\hline 263 & 8164131 & SCAI \\
\hline 264 & 8164810 & RALGDS \\
\hline 265 & 8165094 & QSOX2 \\
\hline 266 & 8165156 & SDCCAG3 \\
\hline 267 & 8165217 & NOTCH1 \\
\hline 268 & 8165552 & NELF \\
\hline 269 & 8165711 & PLCXD1 \\
\hline 270 & 8170364 & AFF2 \\
\hline 271 & 8171837 & KLHL15 \\
\hline 272 & 8172471 & PIM2 \\
\hline 273 & 8173457 & ZMYM3 \\
\hline 274 & 8174692 & SEPT6 \\
\hline 275 & 8176286 & PLCXD1 \\
\hline 276 & 8178404 & MDC1 \\
\hline 277 & 8178727 & ATF6B \\
\hline 278 & 8179884 & EHMT2 \\
\hline 279 & 8180362 & MPRIP \\
\hline 280 & 8180363 & MPRIP \\
\hline 281 & 8180411 & ELMO1 \\
\hline \multicolumn{3}{|c|}{ Cluster 13} \\
\hline 1 & 7899192 & RPS6KA1 \\
\hline 2 & 7900426 & SMAP2 \\
\hline 3 & 7900911 & DPH2 \\
\hline 4 & 7901951 & PGM1 \\
\hline 5 & 7904137 & HIPK1 \\
\hline 6 & 7904742 & LIX1L \\
\hline 7 & 7905444 & SNX27 \\
\hline 8 & 7905631 & INTS3 \\
\hline 9 & 7908779 & RNPEP \\
\hline 10 & 7909708 & CENPF \\
\hline 11 & 7912166 & RERE \\
\hline 12 & 7912257 & CLSTN1 \\
\hline 13 & 7912374 & SRM \\
\hline 14 & 7912412 & MTOR \\
\hline 15 & 7913156 & AKR7A2 \\
\hline 16 & 7914603 & RNF19B \\
\hline 17 & 7915695 & MUTYH \\
\hline 18 & 7919940 & VPS72 \\
\hline 19 & 7919950 & PI4KB \\
\hline 20 & 7919971 & RFX5 \\
\hline 21 & 7920000 & POGZ \\
\hline 22 & 7920707 & FAM189B \\
\hline 23 & 7921133 & HDGF \\
\hline 24 & 7924669 & PYCR2 \\
\hline 25 & 7932109 & SEPHS1 \\
\hline
\end{tabular}

\begin{tabular}{|c|c|c|}
\hline 26 & 7935474 & MMS19 \\
\hline 27 & 7935639 & SLC25A28 \\
\hline 28 & 7937802 & CD81 \\
\hline 29 & 7939215 & C11orf41 \\
\hline 30 & 7940372 & TMEM109 \\
\hline 31 & 7940600 & INCENP \\
\hline 32 & 7941148 & TM7SF2 \\
\hline 33 & 7941179 & CAPN1 \\
\hline 34 & 7941976 & NDUFS8 \\
\hline 35 & 7941985 & TCIRG1 \\
\hline 36 & 7944401 & HMBS \\
\hline 37 & 7945573 & POLR2L \\
\hline 38 & 7947861 & SPI1 \\
\hline 39 & 7949798 & TMEM134 \\
\hline 40 & 7950271 & FAM168A \\
\hline 41 & 7953100 & FKBP4 \\
\hline 42 & 7955736 & ESPL1 \\
\hline 43 & 7956152 & PA2G4 \\
\hline 44 & 7959786 & AACS \\
\hline 45 & 7963646 & AAAS \\
\hline 46 & 7966570 & DDX54 \\
\hline 47 & 7966878 & CIT \\
\hline 48 & 7967544 & SCARB1 \\
\hline 49 & 7968734 & SLC25A15 \\
\hline 50 & 7968890 & DGKZ \\
\hline 51 & 7969438 & LMO7 \\
\hline 52 & 7972577 & RPS26 \\
\hline 53 & 7973067 & PNP \\
\hline 54 & 7975459 & SIPA1L1 \\
\hline 55 & 7975815 & TTLL5 \\
\hline 56 & 7977820 & PRMT5 \\
\hline 57 & 7981494 & AKT1 \\
\hline 58 & 7982878 & CHP \\
\hline 59 & 7983290 & SERF2 \\
\hline 60 & 7983843 & TCF12 \\
\hline 61 & 7985431 & AGSK1 \\
\hline 62 & 7987840 & VPS39 \\
\hline 63 & 7990361 & UBL7 \\
\hline 64 & 7990902 & AGSK1 \\
\hline 65 & 7990952 & AGSK1 \\
\hline 66 & 7991088 & LOC388152 \\
\hline 67 & 7991159 & LOC388152 \\
\hline 68 & 7991714 & AGSK1 \\
\hline 69 & 7992043 & FAM173A \\
\hline 70 & 7992414 & TBL3 \\
\hline 71 & 7992737 & FLYWCH2 \\
\hline 72 & 7995655 & FTO \\
\hline 73 & 7996137 & KATNB1 \\
\hline 74 & 7996685 & EDC4 \\
\hline 75 & 7997453 & PLCG2 \\
\hline 76 & 7998063 & TUBB3 \\
\hline 77 & 7998251 & C16orf13 \\
\hline 78 & 7998267 & JMJD8 \\
\hline
\end{tabular}




\begin{tabular}{|c|c|c|}
\hline 79 & 7998367 & RPUSD1 \\
\hline 80 & 8000869 & SEPT1 \\
\hline 81 & 8003357 & PIEZO1 \\
\hline 82 & 8003733 & SGSM2 \\
\hline 83 & 8004030 & RNF167 \\
\hline 84 & 8004057 & KIF1C \\
\hline 85 & 8005407 & LLGL1 \\
\hline 86 & 8005994 & ERAL1 \\
\hline 87 & 8006655 & DHRS11 \\
\hline 88 & 8007188 & CNP \\
\hline 89 & 8007197 & NKIRAS2 \\
\hline 90 & 8007505 & DHX8 \\
\hline 91 & 8007797 & RPS26 \\
\hline 92 & 8008922 & PPM1D \\
\hline 93 & 8009382 & BPTF \\
\hline 94 & 8010248 & AFMID \\
\hline 95 & 8010664 & MRPL12 \\
\hline 96 & 8010747 & GPS1 \\
\hline 97 & 8011407 & TAX1BP3 \\
\hline 98 & 8013696 & KIAA0100 \\
\hline 99 & 8013788 & FLOT2 \\
\hline 100 & 8014882 & MIEN1 \\
\hline 101 & 8015460 & ACLY \\
\hline 102 & 8015969 & UBTF \\
\hline 103 & 8017460 & SMARCD2 \\
\hline 104 & 8018428 & GALK1 \\
\hline 105 & 8018511 & MRPL38 \\
\hline 106 & 8018849 & TK1 \\
\hline 107 & 8024089 & WDR18 \\
\hline 108 & 8024170 & HMHA1 \\
\hline 109 & 8024204 & STK11 \\
\hline 110 & 8024358 & CSNK1G2 \\
\hline 111 & 8024446 & SPPL2B \\
\hline 112 & 8024497 & THOP1 \\
\hline 113 & 8024660 & HMG20B \\
\hline 114 & 8024864 & HDGFRP2 \\
\hline 115 & 8025255 & STXBP2 \\
\hline 116 & 8026341 & TECR \\
\hline 117 & 8026638 & МYО9В \\
\hline 118 & 8026735 & GTPBP3 \\
\hline 119 & 8026751 & FAM125A \\
\hline 120 & 8027117 & ARMC6 \\
\hline 121 & 8027621 & GPI \\
\hline 122 & 8027701 & GRAMD1A \\
\hline 123 & 8028851 & SHKBP1 \\
\hline 124 & 8028916 & SNRPA \\
\hline 125 & 8029560 & CLPTM1 \\
\hline 126 & 8029814 & PPP5C \\
\hline 127 & 8029831 & CALM3 \\
\hline 128 & 8030113 & RUVBL2 \\
\hline 129 & 8030470 & AP2A1 \\
\hline 130 & 8031097 & NDUFA3 \\
\hline 131 & 8031483 & RPL28 \\
\hline
\end{tabular}

\begin{tabular}{|c|c|c|}
\hline 132 & 8032106 & MED16 \\
\hline 133 & 8033479 & ELAVL1 \\
\hline 134 & 8033899 & EIF3G \\
\hline 135 & 8033956 & S1PR2 \\
\hline 136 & 8034843 & NDUFB7 \\
\hline 137 & 8035553 & COPE \\
\hline 138 & 8036010 & PEPD \\
\hline 139 & 8036525 & MAP4K1 \\
\hline 140 & 8036938 & ADCK4 \\
\hline 141 & 8037537 & ERCC2 \\
\hline 142 & 8037647 & FBXO46 \\
\hline 143 & 8037991 & LIG1 \\
\hline 144 & 8038202 & BCAT2 \\
\hline 145 & 8042867 & WBP1 \\
\hline 146 & 8043377 & WBP1 \\
\hline 147 & 8048304 & CTDSP1 \\
\hline 148 & 8048595 & GMPPA \\
\hline 149 & 8049582 & SCLY \\
\hline 150 & 8051030 & SLC5A6 \\
\hline 151 & 8052803 & AAK1 \\
\hline 152 & 8053599 & WBP1 \\
\hline 153 & 8054092 & TMEM131 \\
\hline 154 & 8054888 & CLASP1 \\
\hline 155 & 8056545 & STK39 \\
\hline 156 & 8059177 & TUBA4A \\
\hline 157 & 8059222 & DNPEP \\
\hline 158 & 8059989 & HES6 \\
\hline 159 & 8060257 & STK25 \\
\hline 160 & 8062319 & TGIF2 \\
\hline 161 & 8064613 & SLC4A11 \\
\hline 162 & 8064739 & C20orf27 \\
\hline 163 & 8065817 & GSS \\
\hline 164 & 8067361 & TAF4 \\
\hline 165 & 8069026 & C21orf 33 \\
\hline 166 & 8071768 & SMARCB1 \\
\hline 167 & 8072744 & NCF4 \\
\hline 168 & 8072870 & PDXP \\
\hline 169 & 8072979 & POLR2F \\
\hline 170 & 8074020 & SELO \\
\hline 171 & 8074388 & SLC25A1 \\
\hline 172 & 8075009 & C22orf13 \\
\hline 173 & 8075217 & AP1B1 \\
\hline 174 & 8076307 & RANGAP1 \\
\hline 175 & 8076393 & CENPM \\
\hline 176 & 8076792 & CERK \\
\hline 177 & 8079311 & LARS2 \\
\hline 178 & 8079563 & DHX30 \\
\hline 179 & 8079950 & GNAI2 \\
\hline 180 & 8080168 & ACY1 \\
\hline 181 & 8080714 & FLNB \\
\hline 182 & 8082422 & EEFSEC \\
\hline 183 & 8086754 & SCAP \\
\hline 184 & 8087790 & RRP9 \\
\hline
\end{tabular}




\begin{tabular}{|c|c|c|}
\hline 185 & 8088106 & TKT \\
\hline 186 & 8092457 & ALG3 \\
\hline 187 & 8093685 & HTT \\
\hline 188 & 8094501 & STIM2 \\
\hline 189 & 8095216 & KIAA1211 \\
\hline 190 & 8101086 & NAAA \\
\hline 191 & 8101622 & TECR \\
\hline 192 & 8103188 & PET112 \\
\hline 193 & 8104506 & TRIO \\
\hline 194 & 8107750 & PRRC1 \\
\hline 195 & 8108330 & KDM3B \\
\hline 196 & 8109999 & ERGIC1 \\
\hline 197 & 8110327 & RGS14 \\
\hline 198 & 8110362 & GRK6 \\
\hline 199 & 8110982 & DAP \\
\hline 200 & 8111286 & DROSHA \\
\hline 201 & 8115524 & CLINT1 \\
\hline 202 & 8119627 & PPP2R5D \\
\hline 203 & 8120585 & SMAP1 \\
\hline 204 & 8124828 & FLOT1 \\
\hline 205 & 8125125 & LSM2 \\
\hline 206 & 8125649 & VPS52 \\
\hline 207 & 8125775 & MNF1 \\
\hline 208 & 8127051 & TRAM2 \\
\hline 209 & 8128111 & UBE2J1 \\
\hline 210 & 8130916 & PHF10 \\
\hline 211 & 8131253 & FOXK1 \\
\hline 212 & 8131427 & C7orf26 \\
\hline 213 & 8133413 & LIMK1 \\
\hline 214 & 8134821 & MEPCE \\
\hline 215 & 8137847 & BRAT1 \\
\hline 216 & 8139281 & POLM \\
\hline 217 & 8139299 & POLD2 \\
\hline 218 & 8139468 & TBRG4 \\
\hline 219 & 8143988 & MLL3 \\
\hline 220 & 8148772 & FAM203A \\
\hline 221 & 8148783 & HEATR7A \\
\hline 222 & 8148799 & FAM203A \\
\hline 223 & 8153652 & SHARPIN \\
\hline 224 & 8153776 & VPS28 \\
\hline 225 & 8153838 & RECQL4 \\
\hline 226 & 8155699 & FXN \\
\hline 227 & 8158123 & FPGS \\
\hline 228 & 8158961 & GTF3C5 \\
\hline 229 & 8160036 & C9orf123 \\
\hline 230 & 8162462 & FAM120A \\
\hline 231 & 8162502 & FBP1 \\
\hline 232 & 8165622 & ZMYND19 \\
\hline 233 & 8165630 & C9orf37 \\
\hline 234 & 8167042 & RBM10 \\
\hline 235 & 8167125 & USP11 \\
\hline 236 & 8167656 & MAGED1 \\
\hline 237 & 8167924 & UBQLN2 \\
\hline
\end{tabular}

\begin{tabular}{|l|l|l|}
\hline $\mathbf{2 3 8}$ & 8170865 & TAZ \\
\hline $\mathbf{2 3 9}$ & 8172119 & MED14 \\
\hline $\mathbf{2 4 0}$ & 8172296 & NDUFB11 \\
\hline $\mathbf{2 4 1}$ & 8173009 & PHF8 \\
\hline $\mathbf{2 4 2}$ & 8175924 & NAA10 \\
\hline $\mathbf{2 4 3}$ & 8176117 & FAM3A \\
\hline $\mathbf{2 4 4}$ & 8178419 & FLOT1 \\
\hline $\mathbf{2 4 5}$ & 8178641 & LSM2 \\
\hline $\mathbf{2 4 6}$ & 8178917 & VPS52 \\
\hline $\mathbf{2 4 7}$ & 8179688 & FLOT1 \\
\hline $\mathbf{2 4 8}$ & 8179839 & LSM2 \\
\hline $\mathbf{2 4 9}$ & 8180123 & VPS52 \\
\hline Cluster 17 & \\
\hline $\mathbf{1}$ & 7898549 & MRTO4 \\
\hline $\mathbf{2}$ & 7899703 & TXLNA \\
\hline $\mathbf{3}$ & 7904000 & DDX20 \\
\hline $\mathbf{4}$ & 7910416 & URB2 \\
\hline $\mathbf{5}$ & 7913805 & RUNX3 \\
\hline $\mathbf{6}$ & 7915091 & MTF1 \\
\hline $\mathbf{7}$ & 7920766 & ASH1L \\
\hline $\mathbf{8}$ & 7923503 & ADIPOR1 \\
\hline $\mathbf{9}$ & 7923778 & ELK4 \\
\hline $\mathbf{1 0}$ & 7924969 & TAF5L \\
\hline $\mathbf{1 1}$ & 7928589 & PPIF \\
\hline $\mathbf{1 2}$ & 7946201 & ARFIP2 \\
\hline $\mathbf{1 3}$ & 7947969 & FNBP4 \\
\hline $\mathbf{1 4}$ & 7948493 & SLC15A3 \\
\hline $\mathbf{1 5}$ & 7949916 & CHKA \\
\hline $\mathbf{1 6}$ & 7967736 & POLE \\
\hline $\mathbf{1 7}$ & 7970111 & ARHGEF7 \\
\hline $\mathbf{1 8}$ & 7971177 & FOXO1 \\
\hline $\mathbf{1 9}$ & 7972548 & GPR18 \\
\hline $\mathbf{2 0}$ & 7974455 & MAPK1IP1L \\
\hline $\mathbf{2 1}$ & 7977105 & TRMT61A \\
\hline $\mathbf{2 2}$ & 7980828 & CCDC88C \\
\hline $\mathbf{2 3}$ & 7982185 & DEXI \\
\hline $\mathbf{2 4}$ & 7986685 & DEXI \\
\hline $\mathbf{2 5}$ & 7987584 & INO80 \\
\hline $\mathbf{2 6}$ & 7988687 & GABPB1 \\
\hline $\mathbf{2 7}$ & 7993148 & PMM2 \\
\hline $\mathbf{2 8}$ & 7993973 & POLR3E \\
\hline $\mathbf{2 9}$ & 7994280 & IL4R \\
\hline $\mathbf{3 0}$ & 7999120 & CORO7 \\
\hline $\mathbf{3 1}$ & 8000244 & USP31 \\
\hline $\mathbf{3 2}$ & 8003249 & FBXO31 \\
\hline $\mathbf{3 3}$ & 8004431 & POLR2A \\
\hline $\mathbf{3 4}$ & 8005441 & SMCR8 \\
\hline $\mathbf{3 5}$ & 8006148 & GOSR1 \\
\hline $\mathbf{3 6}$ & 8006850 & CDK12 \\
\hline $\mathbf{3 7}$ & 8013486 & USP22 \\
\hline $\mathbf{3 8}$ & 8014214 & NLE1 \\
\hline $\mathbf{4 0}$ & 8014925 & MED24 \\
\hline & & \\
\hline
\end{tabular}




\begin{tabular}{|c|c|c|}
\hline 41 & 8021866 & NFATC1 \\
\hline 42 & 8025458 & ZNF317 \\
\hline 43 & 8025563 & PPAN-P2RY11 \\
\hline 44 & 8027074 & UPF1 \\
\hline 45 & 8028119 & WDR62 \\
\hline 46 & 8028552 & NFKBIB \\
\hline 47 & 8030035 & GRWD1 \\
\hline 48 & 8032137 & C19orf6 \\
\hline 49 & 8034482 & TNPO2 \\
\hline 50 & 8035007 & AKAP8 \\
\hline 51 & 8035023 & AKAP8L \\
\hline 52 & 8035980 & RHPN2 \\
\hline 53 & 8037657 & DMPK \\
\hline 54 & 8038624 & C19orf48 \\
\hline 55 & 8042381 & PNO1 \\
\hline 56 & 8042942 & HK2 \\
\hline 57 & 8052143 & GPR75 \\
\hline 58 & 8055183 & SMPD4 \\
\hline 59 & 8059996 & PER2 \\
\hline 60 & 8062286 & C20orf4 \\
\hline 61 & 8066641 & ZNF335 \\
\hline 62 & 8067563 & DIDO1 \\
\hline 63 & 8067593 & YTHDF1 \\
\hline 64 & 8068289 & SON \\
\hline 65 & 8068551 & DYRK1A \\
\hline 66 & 8068810 & SLC37A1 \\
\hline 67 & 8068974 & TRAPPC10 \\
\hline 68 & 8069174 & FAM207A \\
\hline 69 & 8070194 & RUNX1 \\
\hline 70 & 8073039 & GTPBP1 \\
\hline 71 & 8073345 & EP300 \\
\hline 72 & 8074647 & PI4KA \\
\hline 73 & 8075406 & PES1 \\
\hline 74 & 8077595 & BRPF1 \\
\hline 75 & 8080878 & ATXN7 \\
\hline 76 & 8083324 & TSC22D2 \\
\hline 77 & 8087271 & QRICH1 \\
\hline 78 & 8087748 & VPRBP \\
\hline 79 & 8088128 & DCP1A \\
\hline 80 & 8088642 & LRIG1 \\
\hline 81 & 8088776 & FOXP1 \\
\hline 82 & 8093590 & RNF4 \\
\hline 83 & 8097461 & CCRN4L \\
\hline 84 & 8108099 & SEC 24A \\
\hline 85 & 8110841 & LPCAT1 \\
\hline 86 & 8115168 & RBM22 \\
\hline 87 & 8118228 & LY6G5B \\
\hline 88 & 8118580 & BRD2 \\
\hline 89 & 8119712 & SRF \\
\hline 90 & 8130116 & LATS1 \\
\hline 91 & 8133145 & CRCP \\
\hline 92 & 8137542 & RBM33 \\
\hline 93 & 8140061 & BCL7B \\
\hline
\end{tabular}

\begin{tabular}{|c|c|c|}
\hline 94 & 8142730 & ZNF800 \\
\hline 95 & 8145259 & CHMP7 \\
\hline 96 & 8159476 & TRAF2 \\
\hline 97 & 8160647 & BAG1 \\
\hline 98 & 8160722 & UBAP2 \\
\hline 99 & 8161192 & RNF38 \\
\hline 100 & 8162601 & ZNF367 \\
\hline 101 & 8164665 & RAPGEF1 \\
\hline 102 & 8164862 & SURF6 \\
\hline 103 & 8178059 & LY6G5B \\
\hline 104 & 8179504 & BRD2 \\
\hline \multicolumn{3}{|c|}{ Cluster 18} \\
\hline 1 & 7898602 & OTUD3 \\
\hline 2 & 7923659 & PPP1R15B \\
\hline 3 & 7924526 & TP53BP2 \\
\hline 4 & 7925823 & GTPBP4 \\
\hline 5 & 7938331 & ZNF143 \\
\hline 6 & 7939839 & PTPRJ \\
\hline 7 & 7940051 & ZDHHC5 \\
\hline 8 & 7940118 & ZFP91-CNTF \\
\hline 9 & 7942439 & RELT \\
\hline 10 & 7948229 & SLC43A3 \\
\hline 11 & 7955425 & ATF1 \\
\hline 12 & 7965918 & NT5DC3 \\
\hline 13 & 8003448 & ANKRD11 \\
\hline 14 & 8009932 & SAP30BP \\
\hline 15 & 8022404 & FAM210A \\
\hline 16 & 8031956 & ZNF324 \\
\hline 17 & 8069565 & BTG3 \\
\hline 18 & 8077612 & TTLL3 \\
\hline 19 & 8110734 & BRD9 \\
\hline 20 & 8115681 & PANK3 \\
\hline 21 & 8116548 & DUSP22 \\
\hline 22 & 8117128 & E2F3 \\
\hline 23 & 8119408 & NFYA \\
\hline 24 & 8141241 & SMURF1 \\
\hline 25 & 8160756 & DCAF12 \\
\hline 26 & 8162850 & TEX10 \\
\hline 27 & 8173615 & RLIM \\
\hline \multicolumn{3}{|c|}{ Cluster 19} \\
\hline 1 & 7903321 & RTCD1 \\
\hline 2 & 7903619 & SARS \\
\hline 3 & 7903908 & CEPT1 \\
\hline 4 & 7904340 & MAN1A2 \\
\hline 5 & 7906810 & DUSP12 \\
\hline 6 & 7907135 & SFT2D2 \\
\hline 7 & 7914180 & SPCS2 \\
\hline 8 & 7918255 & CLCC1 \\
\hline 9 & 7918284 & TAF13 \\
\hline 10 & 7922250 & SCYL3 \\
\hline 11 & 7923516 & CYB5R1 \\
\hline 12 & 7929012 & STAMBPL1 \\
\hline 13 & 7929634 & ZDHHC16 \\
\hline
\end{tabular}




\begin{tabular}{|c|c|c|}
\hline 14 & 7930162 & C10orf26 \\
\hline 15 & 7930614 & NHLRC2 \\
\hline 16 & 7932160 & FAM107B \\
\hline 17 & 7942168 & FADD \\
\hline 18 & 7942553 & SPCS2 \\
\hline 19 & 7943314 & JRKL \\
\hline 20 & 7945058 & FAM118B \\
\hline 21 & 7946742 & CYP2R1 \\
\hline 22 & 7950753 & CCDC90B \\
\hline 23 & 7953211 & C12orf5 \\
\hline 24 & 7954492 & FGFR1OP2 \\
\hline 25 & 7954711 & C12orf35 \\
\hline 26 & 7958819 & ERP29 \\
\hline 27 & 7959282 & RNF34 \\
\hline 28 & 7964021 & RNF41 \\
\hline 29 & 7964347 & TMEM194A \\
\hline 30 & 7967881 & MPHOSPH8 \\
\hline 31 & 7969626 & GPR180 \\
\hline 32 & 7969979 & ABHD13 \\
\hline 33 & 7971027 & ALG5 \\
\hline 34 & 7973530 & PCK2 \\
\hline 35 & 7974207 & MGAT2 \\
\hline 36 & 7974576 & NAA30 \\
\hline 37 & 7974725 & JKAMP \\
\hline 38 & 7976571 & C14orf129 \\
\hline 39 & 7978932 & SOS2 \\
\hline 40 & 7979984 & ZFYVE1 \\
\hline 41 & 7980438 & SPTLC2 \\
\hline 42 & 7983744 & TMOD3 \\
\hline 43 & 7988753 & SPPL2A \\
\hline 44 & 7989128 & CNOT6L \\
\hline 45 & 7989224 & ADAM10 \\
\hline 46 & 7999553 & CPPED1 \\
\hline 47 & 8002347 & AARS \\
\hline 48 & 8002523 & FTSJD1 \\
\hline 49 & 8018600 & SRP68 \\
\hline 50 & 8021187 & SKA1 \\
\hline 51 & 8021453 & SEC11C \\
\hline 52 & 8035886 & C19orf12 \\
\hline 53 & 8036420 & ZFP30 \\
\hline 54 & 8036813 & ZNF780B \\
\hline 55 & 8040503 & UBXN2A \\
\hline 56 & 8041487 & CCDC75 \\
\hline 57 & 8041820 & SOCS5 \\
\hline 58 & 8042161 & PEX13 \\
\hline 59 & 8044613 & CBWD1 \\
\hline 60 & 8050278 & PDIA6 \\
\hline 61 & 8050474 & RDH14 \\
\hline 62 & 8055890 & STAM2 \\
\hline 63 & 8058221 & TRAK2 \\
\hline 64 & 8058985 & RNF25 \\
\hline 65 & 8061373 & GZF1 \\
\hline 66 & 8071274 & SEPT5-GP1BB \\
\hline
\end{tabular}

\begin{tabular}{|c|c|c|}
\hline 67 & 8077993 & TMEM43 \\
\hline 68 & 8081115 & ARL13B \\
\hline 69 & 8083310 & RNF13 \\
\hline 70 & 8083757 & NMD3 \\
\hline 71 & 8084016 & PIK3CA \\
\hline 72 & 8088065 & SFMBT1 \\
\hline 73 & 8088151 & ACTR8 \\
\hline 74 & 8088700 & TMF1 \\
\hline 75 & 8088830 & SHQ1 \\
\hline 76 & 8090840 & RYK \\
\hline 77 & 8091637 & SLC33A1 \\
\hline 78 & 8091941 & PDCD10 \\
\hline 79 & 8093961 & KIAA0232 \\
\hline 80 & 8095148 & TMEM165 \\
\hline 81 & 8095163 & EXOC1 \\
\hline 82 & 8096251 & NUDT9 \\
\hline 83 & 8097066 & METTL14 \\
\hline 84 & 8097704 & TMEM184C \\
\hline 85 & 8099797 & RELL1 \\
\hline 86 & 8099834 & TLR1 \\
\hline 87 & 8100318 & SGCB \\
\hline 88 & 8103834 & AGA \\
\hline 89 & 8104760 & TARS \\
\hline 90 & 8105862 & CDK7 \\
\hline 91 & 8106303 & POLK \\
\hline 92 & 8106702 & ZCCHC9 \\
\hline 93 & 8106784 & RASA1 \\
\hline 94 & 8107520 & TNFAIP8 \\
\hline 95 & 8108847 & RNF14 \\
\hline 96 & 8110678 & CCDC127 \\
\hline 97 & 8111533 & LMBRD2 \\
\hline 98 & 8111952 & C5orf28 \\
\hline 99 & 8112182 & MIER3 \\
\hline 100 & 8112687 & COL4A3BP \\
\hline 101 & 8114829 & YIPF5 \\
\hline 102 & 8115476 & MED7 \\
\hline 103 & 8116998 & JARID2 \\
\hline 104 & 8118613 & SLC39A7 \\
\hline 105 & 8120215 & PAQR8 \\
\hline 106 & 8120378 & KIAA1586 \\
\hline 107 & 8121525 & KIAA1919 \\
\hline 108 & 8121861 & NCOA7 \\
\hline 109 & 8121927 & RNF146 \\
\hline 110 & 8129254 & MAN1A1 \\
\hline 111 & 8130032 & FBXO30 \\
\hline 112 & 8130438 & TFB1M \\
\hline 113 & 8130765 & FAM103A1 \\
\hline 114 & 8132292 & Sep-07 \\
\hline 115 & 8133167 & KCTD7 \\
\hline 116 & 8135464 & DLD \\
\hline 117 & 8137464 & PSPH \\
\hline 118 & 8137709 & ZFAND2A \\
\hline 119 & 8141150 & ASNS \\
\hline
\end{tabular}




\begin{tabular}{|c|c|c|}
\hline 120 & 8142540 & FAM3C \\
\hline 121 & 8144774 & VPS37A \\
\hline 122 & 8146000 & ADAM9 \\
\hline 123 & 8146649 & MTFR1 \\
\hline 124 & 8148658 & ZNF623 \\
\hline 125 & 8150219 & BRF2 \\
\hline 126 & 8150565 & RNF170 \\
\hline 127 & 8151436 & PEX2 \\
\hline 128 & 8151788 & RBM12B \\
\hline 129 & 8155214 & MELK \\
\hline 130 & 8155422 & CBWD5 \\
\hline 131 & 8155636 & CBWD3 \\
\hline 132 & 8156043 & PSAT1 \\
\hline 133 & 8158513 & DOLPP1 \\
\hline 134 & 8159815 & CBWD1 \\
\hline 135 & 8160405 & KLHL9 \\
\hline 136 & 8161537 & CBWD3 \\
\hline 137 & 8161587 & CBWD3 \\
\hline 138 & 8166098 & RAB9A \\
\hline 139 & 8166140 & MOSPD2 \\
\hline 140 & 8166442 & FAM3C \\
\hline 141 & 8169617 & PGRMC1 \\
\hline 142 & 8176230 & RAB39B \\
\hline 143 & 8177462 & CDK7 \\
\hline 144 & 8178225 & SLC39A7 \\
\hline 145 & 8179525 & SLC39A7 \\
\hline 146 & 8180397 & NT5C3 \\
\hline \multicolumn{3}{|c|}{ Cluster 20 } \\
\hline 1 & 7899173 & DHDDS \\
\hline 2 & 7899377 & PPP1R8 \\
\hline 3 & 7899486 & TRNAU1AP \\
\hline 4 & 7901662 & TTC4 \\
\hline 5 & 7904883 & CHD1L \\
\hline 6 & 7907124 & TIPRL \\
\hline 7 & 7907445 & KLHL20 \\
\hline 8 & 7913242 & MUL1 \\
\hline 9 & 7922008 & TADA1 \\
\hline 10 & 7922391 & CENPL \\
\hline 11 & 7926319 & SUV39H2 \\
\hline 12 & 7928752 & GHITM \\
\hline 13 & 7929831 & HIF1AN \\
\hline 14 & 7933115 & SEPT7L \\
\hline 15 & 7934852 & GLUD1 \\
\hline 16 & 7939329 & PDHX \\
\hline 17 & 7943218 & PANX1 \\
\hline 18 & 7943721 & FDX1 \\
\hline 19 & 7943842 & C11orf57 \\
\hline 20 & 7954279 & AEBP2 \\
\hline 21 & 7954382 & PYROXD1 \\
\hline 22 & 7959657 & ATP6V0A2 \\
\hline 23 & 7961829 & BCAT1 \\
\hline 24 & 7962185 & AMN1 \\
\hline 25 & 7967287 & ZCCHC8 \\
\hline
\end{tabular}

\begin{tabular}{|c|c|c|}
\hline 26 & 7970084 & CARKD \\
\hline 27 & 7970716 & LNX2 \\
\hline 28 & 7971550 & MED4 \\
\hline 29 & 7974473 & FBXO34 \\
\hline 30 & 7975136 & FUT8 \\
\hline 31 & 7977127 & KLC1 \\
\hline 32 & 7978166 & TM9SF1 \\
\hline 33 & 7980523 & GTF2A1 \\
\hline 34 & 7988245 & MFAP1 \\
\hline 35 & 7989516 & HERC1 \\
\hline 36 & 7993833 & METTL9 \\
\hline 37 & 7997230 & PSMD7 \\
\hline 38 & 8001317 & N4BP1 \\
\hline 39 & 8004111 & RABEP1 \\
\hline 40 & 8004144 & MIS12 \\
\hline 41 & 8005512 & PRPSAP2 \\
\hline 42 & 8006477 & ZNF830 \\
\hline 43 & 8007435 & RUNDC1 \\
\hline 44 & 8015456 & KLHL11 \\
\hline 45 & 8017235 & APPBP2 \\
\hline 46 & 8021208 & ME2 \\
\hline 47 & 8023246 & C18orf32 \\
\hline 48 & 8023481 & NARS \\
\hline 49 & 8026339 & SNRPG \\
\hline 50 & 8027510 & C19orf40 \\
\hline 51 & 8027521 & GPATCH1 \\
\hline 52 & 8030991 & LOC147804 \\
\hline 53 & 8035193 & C19orf42 \\
\hline 54 & 8042830 & MTHFD2 \\
\hline 55 & 8048847 & AGFG1 \\
\hline 56 & 8053046 & DUSP11 \\
\hline 57 & 8054978 & ERCC3 \\
\hline 58 & 8061129 & C20orf72 \\
\hline 59 & 8061262 & NAA20 \\
\hline 60 & 8070215 & SETD4 \\
\hline 61 & 8073875 & TRMU \\
\hline 62 & 8079869 & RBM5 \\
\hline 63 & 8084064 & MTHFD2 \\
\hline 64 & 8084904 & SDHAP2 \\
\hline 65 & 8084912 & SDHAP2 \\
\hline 66 & 8087634 & TUSC2 \\
\hline 67 & 8092534 & TMEM41A \\
\hline 68 & 8092905 & LSG1 \\
\hline 69 & 8093039 & SDHAP1 \\
\hline 70 & 8097570 & USP38 \\
\hline 71 & 8101228 & CNOT6L \\
\hline 72 & 8104422 & MTRR \\
\hline 73 & 8113250 & ERAP1 \\
\hline 74 & 8113591 & PGGT1B \\
\hline 75 & 8116867 & TMEM14B \\
\hline 76 & 8116969 & NOL7 \\
\hline 77 & 8120698 & MTO1 \\
\hline 78 & 8124262 & TDP2 \\
\hline
\end{tabular}




\begin{tabular}{|l|l|l|}
\hline $\mathbf{7 9}$ & 8128650 & SEC63 \\
\hline $\mathbf{8 0}$ & 8131356 & CCZ1 \\
\hline $\mathbf{8 1}$ & 8132458 & MRPL32 \\
\hline $\mathbf{8 2}$ & 8134581 & ARPC1A \\
\hline $\mathbf{8 3}$ & 8134621 & ZKSCAN5 \\
\hline $\mathbf{8 4}$ & 8135064 & TRIM56 \\
\hline $\mathbf{8 5}$ & 8138128 & CCZ1 \\
\hline $\mathbf{8 6}$ & 8141133 & SHFM1 \\
\hline $\mathbf{8 7}$ & 8141169 & MGC72080 \\
\hline $\mathbf{8 8}$ & 8144528 & TNKS \\
\hline $\mathbf{8 9}$ & 8144931 & ATP6V1B2 \\
\hline $\mathbf{9 0}$ & 8146448 & MRPL15 \\
\hline $\mathbf{9 1}$ & 8146544 & UBXN2B \\
\hline $\mathbf{9 2}$ & 8146930 & TMEM70 \\
\hline $\mathbf{9 3}$ & 8148941 & ZNF7 \\
\hline $\mathbf{9 4}$ & 8151118 & VCPIP1 \\
\hline $\mathbf{9 5}$ & 8155234 & ZCCHC7 \\
\hline $\mathbf{9 6}$ & 8157700 & RABGAP1 \\
\hline $\mathbf{9 7}$ & 8158112 & CDK9 \\
\hline $\mathbf{9 8}$ & 8162676 & TSTD2 \\
\hline $\mathbf{9 9}$ & 8163402 & PTBP3 \\
\hline $\mathbf{1 0 0}$ & 8163784 & FBXW2 \\
\hline $\mathbf{1 0 1}$ & 8163964 & PDCL \\
\hline $\mathbf{1 0 2}$ & 8169920 & RBMX2 \\
\hline $\mathbf{1 0 3}$ & 8178090 & C6orf48 \\
\hline $\mathbf{1 0 4}$ & 8179326 & C6orf48 \\
\hline $\mathbf{1 0 5}$ & 8180257 & CEP170 \\
\hline Cluster 21 & \\
\hline $\mathbf{1}$ & 7897370 & VAMP3 \\
\hline $\mathbf{2}$ & 7905171 & PRPF3 \\
\hline $\mathbf{3}$ & 7906400 & IFI16 \\
\hline $\mathbf{4}$ & 7909782 & RRP15 \\
\hline $\mathbf{5}$ & 7911539 & CCNL2 \\
\hline $\mathbf{6}$ & 7922432 & RC3H1 \\
\hline $\mathbf{7}$ & 7925996 & FBXO18 \\
\hline $\mathbf{8}$ & 7926916 & ZEB1 \\
\hline $\mathbf{9}$ & 7927233 & FAM21C \\
\hline $\mathbf{1 0}$ & 7927323 & FAM21B \\
\hline $\mathbf{1 1}$ & 7927560 & FAM21A \\
\hline $\mathbf{1 2}$ & 7935425 & RRP12 \\
\hline $\mathbf{1 3}$ & 7936596 & C10orf46 \\
\hline $\mathbf{1 4}$ & 7946635 & ZBED5 \\
\hline $\mathbf{1 5}$ & 7947894 & CELF1 \\
\hline $\mathbf{1 6}$ & 7953594 & EMG1 \\
\hline $\mathbf{1 7}$ & 7956524 & PIP4K2C \\
\hline $\mathbf{1 8}$ & 7966202 & KCTD10 \\
\hline $\mathbf{1 9}$ & 7968999 & NUDT15 \\
\hline $\mathbf{2 0}$ & 7970858 & HMGB1 \\
\hline $\mathbf{2 3}$ & 7989998 & BTBD7 \\
\hline & 7993341 & MEF2A \\
\hline
\end{tabular}

\begin{tabular}{|c|c|c|}
\hline 26 & 8003298 & SLC7A5 \\
\hline 27 & 8006183 & SUZ12P \\
\hline 28 & 8007904 & GOSR2 \\
\hline 29 & 8008530 & UTP18 \\
\hline 30 & 8014738 & CWC25 \\
\hline 31 & 8021228 & SMAD4 \\
\hline 32 & 8031899 & ERVK3-1 \\
\hline 33 & 8039909 & CCNL2 \\
\hline 34 & 8043105 & KCMF1 \\
\hline 35 & 8048940 & SP100 \\
\hline 36 & 8053388 & TGOLN2 \\
\hline 37 & 8053562 & CHMP3 \\
\hline 38 & 8061715 & KIF3B \\
\hline 39 & 8063211 & NCOA3 \\
\hline 40 & 8063315 & DDX27 \\
\hline 41 & 8063607 & RAB22A \\
\hline 42 & 8064976 & DNAJC9 \\
\hline 43 & 8067206 & CTCFL \\
\hline 44 & 8073733 & NUP50 \\
\hline 45 & 8077931 & MKRN2 \\
\hline 46 & 8082408 & SEC61A1 \\
\hline 47 & 8087461 & GMPPB \\
\hline 48 & 8091764 & KPNA4 \\
\hline 49 & 8099029 & TNIP2 \\
\hline 50 & 8103922 & CASP3 \\
\hline 51 & 8107353 & ZRSR1 \\
\hline 52 & 8107578 & SRFBP1 \\
\hline 53 & 8108510 & $\begin{array}{l}\text { ANKHD1- } \\
\text { EIF4EBP3 }\end{array}$ \\
\hline 54 & 8109528 & CYFIP2 \\
\hline 55 & 8110169 & FAF2 \\
\hline 56 & 8110894 & NSUN2 \\
\hline 57 & 8112312 & DIMT1 \\
\hline 58 & 8115765 & FBXW11 \\
\hline 59 & 8115783 & STK10 \\
\hline 60 & 8115895 & KIAA1191 \\
\hline 61 & 8122672 & TAB2 \\
\hline 62 & 8124022 & DTNBP1 \\
\hline 63 & 8124950 & LTB \\
\hline 64 & 8125850 & C6orf106 \\
\hline 65 & 8130383 & MTRF1L \\
\hline 66 & 8131044 & GET4 \\
\hline 67 & 8132188 & AVL9 \\
\hline 68 & 8134992 & SRRT \\
\hline 69 & 8135363 & PIK3CG \\
\hline 70 & 8136539 & LUC7L2 \\
\hline 71 & 8139859 & GUSB \\
\hline 72 & 8142852 & TNPO3 \\
\hline 73 & 8144586 & MTMR9 \\
\hline 74 & 8145922 & DDHD2 \\
\hline 75 & 8147785 & DCAF13 \\
\hline 76 & 8150287 & WHSC1L1 \\
\hline 77 & 8150537 & SLC20A2 \\
\hline
\end{tabular}




\begin{tabular}{|c|c|c|}
\hline 78 & 8152628 & DERL1 \\
\hline 79 & 8154012 & KANK1 \\
\hline 80 & 8154856 & UBE2R2 \\
\hline 81 & 8157945 & ZBTB34 \\
\hline 82 & 8158686 & FUBP3 \\
\hline 83 & 8161632 & PTAR1 \\
\hline 84 & 8164587 & TOR1A \\
\hline 85 & 8167790 & TSR2 \\
\hline 86 & 8177560 & BDP1 \\
\hline 87 & 8178512 & LTB \\
\hline 88 & 8179768 & LTB \\
\hline \multicolumn{3}{|c|}{ Cluster 22} \\
\hline 1 & 7900438 & ZNF643 \\
\hline 2 & 7902166 & MIER1 \\
\hline 3 & 7903369 & SLC30A7 \\
\hline 4 & 7903893 & CD53 \\
\hline 5 & 7906819 & ATF6 \\
\hline 6 & 7907773 & TOR1AIP1 \\
\hline 7 & 7909628 & FLVCR1 \\
\hline 8 & 7912675 & ZBTB17 \\
\hline 9 & 7914202 & SNHG12 \\
\hline 10 & 7914214 & SNORA44 \\
\hline 11 & 7916135 & CC2D1B \\
\hline 12 & 7917771 & DNTTIP2 \\
\hline 13 & 7919888 & CDC42SE1 \\
\hline 14 & 7920839 & RIT1 \\
\hline 15 & 7921667 & CD48 \\
\hline 16 & 7921970 & ALDH9A1 \\
\hline 17 & 7924701 & ACBD3 \\
\hline 18 & 7926021 & RBM17 \\
\hline 19 & 7926836 & RAB18 \\
\hline 20 & 7927513 & FAM21C \\
\hline 21 & 7927767 & $\mathrm{ADO}$ \\
\hline 22 & 7927972 & VPS26A \\
\hline 23 & 7928171 & SGPL1 \\
\hline 24 & 7932885 & ARHGAP12 \\
\hline 25 & 7934122 & SAR1A \\
\hline 26 & 7934459 & NDST2 \\
\hline 27 & 7934812 & WAPAL \\
\hline 28 & 7936463 & ABLIM1 \\
\hline 29 & 7938762 & GTF2H1 \\
\hline 30 & 7947540 & TRAF6 \\
\hline 31 & 7953021 & ADIPOR2 \\
\hline 32 & 7959473 & DENR \\
\hline 33 & 7960134 & ZNF26 \\
\hline 34 & 7962516 & SLC38A1 \\
\hline 35 & 7966638 & RBM19 \\
\hline 36 & 7967624 & SLC15A4 \\
\hline 37 & 7967794 & ANKLE2 \\
\hline 38 & 7970413 & PSPC1 \\
\hline 39 & 7970696 & USP12 \\
\hline 40 & 7972062 & FBXL3 \\
\hline 41 & 7972828 & ANKRD10 \\
\hline
\end{tabular}

\begin{tabular}{|c|c|c|}
\hline 42 & 7974249 & ARF6 \\
\hline 43 & 7975645 & ZNF410 \\
\hline 44 & 7975863 & C14orf118 \\
\hline 45 & 7978628 & PPP2R3C \\
\hline 46 & 7979044 & NIN \\
\hline 47 & 7979223 & DDHD1 \\
\hline 48 & 7980680 & FOXN3 \\
\hline 49 & 7980833 & SMEK1 \\
\hline 50 & 7986250 & CHD2 \\
\hline 51 & 7986665 & NIPA2 \\
\hline 52 & 7986675 & NIPA1 \\
\hline 53 & 7989013 & RSL24D1 \\
\hline 54 & 7991581 & CHSY1 \\
\hline 55 & 7993159 & C16orf72 \\
\hline 56 & 7993433 & PDXDC1 \\
\hline 57 & 7994102 & DCTN5 \\
\hline 58 & 7994518 & SPNS1 \\
\hline 59 & 7996341 & PDP2 \\
\hline 60 & 7996772 & SLC7A6 \\
\hline 61 & 7997381 & CENPN \\
\hline 62 & 7998174 & LUC7L \\
\hline 63 & 8001841 & DYNC1LI2 \\
\hline 64 & 8002878 & TMEM170A \\
\hline 65 & 8003758 & PAFAH1B1 \\
\hline 66 & 8007885 & NSF \\
\hline 67 & 8008087 & NFE2L1 \\
\hline 68 & 8008139 & UBE2Z \\
\hline 69 & 8013908 & NUFIP2 \\
\hline 70 & 8015642 & PSMC3IP \\
\hline 71 & 8017711 & GNA13 \\
\hline 72 & 8020149 & NAPG \\
\hline 73 & 8021113 & C18orf25 \\
\hline 74 & 8022996 & EPG5 \\
\hline 75 & 8042291 & AFTPH \\
\hline 76 & 8045398 & RAB3GAP1 \\
\hline 77 & 8046726 & SSFA2 \\
\hline 78 & 8047865 & PIKFYVE \\
\hline 79 & 8048752 & MRPL44 \\
\hline 80 & 8049657 & ASB1 \\
\hline 81 & 8050128 & KIDINS220 \\
\hline 82 & 8050190 & ADAM17 \\
\hline 83 & 8050719 & ITSN2 \\
\hline 84 & 8052024 & FBXO11 \\
\hline 85 & 8052680 & RAB1A \\
\hline 86 & 8053668 & EIF2AK3 \\
\hline 87 & 8054227 & REV1 \\
\hline 88 & 8054804 & CCDC93 \\
\hline 89 & 8054997 & MAP3K2 \\
\hline 90 & 8058182 & FAM126B \\
\hline 91 & 8068062 & USP16 \\
\hline 92 & 8068919 & PDXK \\
\hline 93 & 8070269 & DSCR3 \\
\hline 94 & 8072454 & RNF185 \\
\hline
\end{tabular}




\begin{tabular}{|c|c|c|}
\hline 95 & 8075130 & PITPNB \\
\hline 96 & 8077450 & ARL8B \\
\hline 97 & 8077458 & EDEM1 \\
\hline 98 & 8078350 & TGFBR2 \\
\hline 99 & 8078569 & GOLGA4 \\
\hline 100 & 8079021 & CTNNB1 \\
\hline 101 & 8081055 & CHMP2B \\
\hline 102 & 8084717 & ST6GAL1 \\
\hline 103 & 8087473 & IP6K1 \\
\hline 104 & 8089299 & CD47 \\
\hline 105 & 8089584 & C3orf17 \\
\hline 106 & 8089999 & KPNA1 \\
\hline 107 & 8090490 & RPN1 \\
\hline 108 & 8092392 & KLHL6 \\
\hline 109 & 8092849 & ATP13A3 \\
\hline 110 & 8096938 & LARP7 \\
\hline 111 & 8098414 & SPCS3 \\
\hline 112 & 8099850 & TMEM156 \\
\hline 113 & 8101013 & RCHY1 \\
\hline 114 & 8103524 & TMEM192 \\
\hline 115 & 8105681 & ERBB2IP \\
\hline 116 & 8107194 & C5orf30 \\
\hline 117 & 8109062 & FBXO38 \\
\hline 118 & 8109344 & GM2A \\
\hline 119 & 8109350 & SLC36A1 \\
\hline 120 & 8110886 & MED10 \\
\hline 121 & 8111129 & ZNF622 \\
\hline 122 & 8113023 & TMEM161B \\
\hline 123 & 8113616 & FEM1C \\
\hline 124 & 8114083 & AFF4 \\
\hline 125 & 8114326 & FAM13B \\
\hline 126 & 8114787 & GNPDA1 \\
\hline 127 & 8114814 & NR3C1 \\
\hline 128 & 8115022 & CSNK1A1 \\
\hline 129 & 8122202 & MYB \\
\hline 130 & 8127425 & LMBRD1 \\
\hline 131 & 8128260 & MAP3К7 \\
\hline 132 & 8130071 & C15orf29 \\
\hline 133 & 8131292 & RBAK \\
\hline 134 & 8132070 & GARS \\
\hline 135 & 8133176 & RABGEF1 \\
\hline 136 & 8133914 & DMTF1 \\
\hline 137 & 8135955 & CALU \\
\hline 138 & 8136259 & MKLN1 \\
\hline 139 & 8137833 & SNX8 \\
\hline 140 & 8138566 & IGF2BP3 \\
\hline 141 & 8138592 & TRA2A \\
\hline 142 & 8138613 & OSBPL3 \\
\hline 143 & 8139592 & HUS1 \\
\hline 144 & 8139891 & SBDS \\
\hline 145 & 8140311 & PMS2P3 \\
\hline 146 & 8143697 & ZNF786 \\
\hline 147 & 8144516 & ERI1 \\
\hline
\end{tabular}

\begin{tabular}{|c|c|c|}
\hline 148 & 8144658 & NEIL2 \\
\hline 149 & 8147548 & POP1 \\
\hline 150 & 8149258 & MFHAS1 \\
\hline 151 & 8149707 & BIN3 \\
\hline 152 & 8150126 & PPP2CB \\
\hline 153 & 8150797 & ATP6V1H \\
\hline 154 & 8152845 & FAM49B \\
\hline 155 & 8158560 & TOR1B \\
\hline 156 & 8162294 & SPTLC1 \\
\hline 157 & 8167573 & GAGE1 \\
\hline 158 & 8175360 & MMGT1 \\
\hline 159 & 8176698 & TXLNG2P \\
\hline 160 & 8177137 & UTY \\
\hline 161 & 8180323 & USP12 \\
\hline \multicolumn{3}{|c|}{ Cluster 23} \\
\hline 1 & 7897322 & PHF13 \\
\hline 2 & 7897774 & CLCN6 \\
\hline 3 & 7899436 & SESN2 \\
\hline 4 & 7901054 & PLK3 \\
\hline 5 & 7902290 & CTH \\
\hline 6 & 7902400 & SNORD45B \\
\hline 7 & 7906564 & PEA15 \\
\hline 8 & 7907171 & BLZF1 \\
\hline 9 & 7910014 & CNIH4 \\
\hline 10 & 7910427 & GALNT2 \\
\hline 11 & 7911754 & TNFRSF14 \\
\hline 12 & 7914212 & SNORA61 \\
\hline 13 & 7915160 & RRAGC \\
\hline 14 & 7915392 & HIVEP3 \\
\hline 15 & 7918157 & VAV3 \\
\hline 16 & 7918902 & CD58 \\
\hline 17 & 7923141 & DENND1B \\
\hline 18 & 7923406 & PTPN7 \\
\hline 19 & 7924760 & ITPKB \\
\hline 20 & 7927146 & CSGALNACT2 \\
\hline 21 & 7928308 & DDIT4 \\
\hline 22 & 7930927 & INPP5F \\
\hline 23 & 7931353 & PTPRE \\
\hline 24 & 7931479 & INPP5A \\
\hline 25 & 7933877 & JMJD1C \\
\hline 26 & 7940160 & DTX4 \\
\hline 27 & 7940191 & STX3 \\
\hline 28 & 7942289 & RNF121 \\
\hline 29 & 7943293 & ENDOD1 \\
\hline 30 & 7948332 & LPXN \\
\hline 31 & 7948364 & MPEG1 \\
\hline 32 & 7950743 & RAB30 \\
\hline 33 & 7953878 & CLEC2D \\
\hline 34 & 7957665 & ELK3 \\
\hline 35 & 7958884 & OAS1 \\
\hline 36 & 7960637 & LPAR5 \\
\hline 37 & 7960933 & M6PR \\
\hline 38 & 7961151 & KLRK1 \\
\hline
\end{tabular}




\begin{tabular}{|c|c|c|}
\hline 39 & 7961371 & DUSP16 \\
\hline 40 & 7963174 & CERS5 \\
\hline 41 & 7964460 & DDIT3 \\
\hline 42 & 7965094 & E2F7 \\
\hline 43 & 7965964 & SLC41A2 \\
\hline 44 & 7968976 & LRCH1 \\
\hline 45 & 7969060 & FNDC3A \\
\hline 46 & 7969651 & DNAJC3 \\
\hline 47 & 7970844 & KATNAL1 \\
\hline 48 & 7975068 & ZBTB1 \\
\hline 49 & 7980547 & SEL1L \\
\hline 50 & 7982564 & SPRED1 \\
\hline 51 & 7983828 & TEX9 \\
\hline 52 & 7984112 & RAB8B \\
\hline 53 & 7985482 & WHAMM \\
\hline 54 & 7989069 & PYGO1 \\
\hline 55 & 7990345 & SEMA7A \\
\hline 56 & 7992789 & TNFRSF12A \\
\hline 57 & 7993167 & ATF7IP2 \\
\hline 58 & 7995895 & HERPUD1 \\
\hline 59 & 7996022 & CCL22 \\
\hline 60 & 7996954 & NFAT5 \\
\hline 61 & 8000028 & DCUN1D3 \\
\hline 62 & 8002322 & PDXDC2P \\
\hline 63 & 8003611 & FAM57A \\
\hline 64 & 8003875 & SPNS2 \\
\hline 65 & 8005707 & MAP2K3 \\
\hline 66 & 8005765 & WSB1 \\
\hline 67 & 8006123 & CPD \\
\hline 68 & 8011875 & DERL2 \\
\hline 69 & 8013071 & FLCN \\
\hline 70 & 8014233 & SLFN11 \\
\hline 71 & 8014391 & CCL3L1 \\
\hline 72 & 8014414 & CCL3L1 \\
\hline 73 & 8016088 & CCDC43 \\
\hline 74 & 8017843 & SLC16A6 \\
\hline 75 & 8019308 & MAFG \\
\hline 76 & 8019731 & CCL3L1 \\
\hline 77 & 8019796 & MAFG \\
\hline 78 & 8023646 & BCL2 \\
\hline 79 & 8026272 & IL27RA \\
\hline 80 & 8027837 & CD22 \\
\hline 81 & 8032410 & MOB3A \\
\hline 82 & 8035694 & PBX4 \\
\hline 83 & 8040440 & GEN1 \\
\hline 84 & 8042391 & PLEK \\
\hline 85 & 8042416 & ARHGAP25 \\
\hline 86 & 8045289 & RHOQ \\
\hline 87 & 8047854 & CCNYL1 \\
\hline 88 & 8050766 & ADCY3 \\
\hline 89 & 8051547 & PRKD3 \\
\hline 90 & 8056977 & NFE2L2 \\
\hline 91 & 8058824 & MREG \\
\hline
\end{tabular}

\begin{tabular}{|c|c|c|}
\hline 92 & 8059361 & WDFY1 \\
\hline 93 & 8059854 & ARL4C \\
\hline 94 & 8060344 & TRIB3 \\
\hline 95 & 8060418 & SIRPA \\
\hline 96 & 8062964 & SYS1 \\
\hline 97 & 8063923 & SLCO4A1 \\
\hline 98 & 8064808 & SLC23A2 \\
\hline 99 & 8064868 & GPCPD1 \\
\hline 100 & 8067113 & ZNF217 \\
\hline 101 & 8069532 & HSPA13 \\
\hline 102 & 8071559 & SDF2L1 \\
\hline 103 & 8076533 & PACSIN2 \\
\hline 104 & 8079517 & KLHL18 \\
\hline 105 & 8082075 & DTX3L \\
\hline 106 & 8083090 & ZBTB38 \\
\hline 107 & 8084045 & MFN1 \\
\hline 108 & 8084634 & DNAJB11 \\
\hline 109 & 8090018 & PARP9 \\
\hline 110 & 8096004 & BMP2K \\
\hline 111 & 8096675 & TET2 \\
\hline 112 & 8097017 & UGT8 \\
\hline 113 & 8099760 & ARAP2 \\
\hline 114 & 8099897 & UGDH \\
\hline 115 & 8101925 & LAMTOR3 \\
\hline 116 & 8102371 & C4orf21 \\
\hline 117 & 8102389 & C4orf 21 \\
\hline 118 & 8103951 & ACSL1 \\
\hline 119 & 8104499 & ANKRD33B \\
\hline 120 & 8105067 & PTGER4 \\
\hline 121 & 8106252 & HEXB \\
\hline 122 & 8110569 & SQSTM1 \\
\hline 123 & 8112409 & SGTB \\
\hline 124 & 8112428 & CD180 \\
\hline 125 & 8113064 & LYSMD3 \\
\hline 126 & 8113914 & FNIP1 \\
\hline 127 & 8118137 & LTA \\
\hline 128 & 8118322 & SNORD52 \\
\hline 129 & 8119088 & CDKN1A \\
\hline 130 & 8122343 & HECA \\
\hline 131 & 8122756 & PLEKHG1 \\
\hline 132 & 8123181 & IGF2R \\
\hline 133 & 8123609 & SERPINB9 \\
\hline 134 & 8123976 & GFOD1 \\
\hline 135 & 8125461 & HLA-DQB 1 \\
\hline 136 & 8126542 & ZNF318 \\
\hline 137 & 8126666 & NFKBIE \\
\hline 138 & 8128716 & CD164 \\
\hline 139 & 8129804 & MAP3K5 \\
\hline 140 & 8129861 & IFNGR1 \\
\hline 141 & 8131265 & KIAA0415 \\
\hline 142 & 8131479 & MIOS \\
\hline 143 & 8132305 & EEPD1 \\
\hline 144 & 8135514 & IFRD1 \\
\hline
\end{tabular}




\begin{tabular}{|c|c|c|}
\hline 145 & 8135568 & MDFIC \\
\hline 146 & 8135576 & TES \\
\hline 147 & 8136591 & ADCK2 \\
\hline 148 & 8137091 & ZNF398 \\
\hline 149 & 8139003 & HERPUD2 \\
\hline 150 & 8145136 & PPP3CC \\
\hline 151 & 8145954 & TACC1 \\
\hline 152 & 8147344 & PDP1 \\
\hline 153 & 8147439 & PLEKHF2 \\
\hline 154 & 8149330 & CTSB \\
\hline 155 & 8149534 & ASAH1 \\
\hline 156 & 8152041 & RNF19A \\
\hline 157 & 8153201 & EIF2C2 \\
\hline 158 & 8155930 & GCNT1 \\
\hline 159 & 8157524 & TLR4 \\
\hline 160 & 8157933 & ZBTB43 \\
\hline 161 & 8159554 & UAP1L1 \\
\hline 162 & 8163825 & TRAF1 \\
\hline 163 & 8164165 & HSPA5 \\
\hline 164 & 8164343 & FAM102A \\
\hline 165 & 8164701 & SETX \\
\hline 166 & 8167006 & RP2 \\
\hline 167 & 8168447 & CXorf26 \\
\hline 168 & 8177976 & LTA \\
\hline 169 & 8179258 & LTA \\
\hline \multicolumn{3}{|c|}{ Cluster 24} \\
\hline 1 & 7899310 & TMEM222 \\
\hline 2 & 7900146 & ZC3H12A \\
\hline 3 & 7907690 & TOR3A \\
\hline 4 & 7912102 & KLHL21 \\
\hline 5 & 7913357 & ECE1 \\
\hline 6 & 7914021 & SLC9A1 \\
\hline 7 & 7920944 & SMG5 \\
\hline 8 & 7922717 & RGS16 \\
\hline 9 & 7923394 & ARL8A \\
\hline 10 & 7926531 & ARL5B \\
\hline 11 & 7930074 & NFKB2 \\
\hline 12 & 7948399 & PATL1 \\
\hline 13 & 7958749 & SH2B3 \\
\hline 14 & 7979813 & ZFP36L1 \\
\hline 15 & 7980051 & C14orf43 \\
\hline 16 & 7984952 & C15orf39 \\
\hline 17 & 7995258 & ZNF267 \\
\hline 18 & 8004671 & KDM6B \\
\hline 19 & 8004691 & TMEM88 \\
\hline 20 & 8013312 & FAM83G \\
\hline 21 & 8014956 & NR1D1 \\
\hline 22 & 8018937 & USP36 \\
\hline 23 & 8024228 & MIDN \\
\hline 24 & 8026350 & CLEC17A \\
\hline 25 & 8026915 & ARRDC2 \\
\hline 26 & 8030128 & PPP1R15A \\
\hline 27 & 8035703 & LPAR2 \\
\hline
\end{tabular}

\begin{tabular}{|c|c|c|}
\hline 28 & 8036207 & NFKBID \\
\hline 29 & 8040103 & ID2 \\
\hline 30 & 8049075 & B3GNT7 \\
\hline 31 & 8053882 & DUSP2 \\
\hline 32 & 8060427 & STK35 \\
\hline 33 & 8063156 & CD40 \\
\hline 34 & 8063583 & RBM38 \\
\hline 35 & 8066905 & ZNFX1 \\
\hline 36 & 8068280 & IFNGR2 \\
\hline 37 & 8071899 & ADORA2A \\
\hline 38 & 8073960 & PIM3 \\
\hline 39 & 8077728 & LOC442075 \\
\hline 40 & 8082314 & PLXNA1 \\
\hline 41 & 8086330 & CSRNP1 \\
\hline 42 & 8091485 & SIAH2 \\
\hline 43 & 8096635 & NFKB 1 \\
\hline 44 & 8103025 & ZNF827 \\
\hline 45 & 8109201 & HMGXB3 \\
\hline 46 & 8113761 & ZNF608 \\
\hline 47 & 8115806 & UBTD2 \\
\hline 48 & 8116559 & IRF4 \\
\hline 49 & 8116983 & CD83 \\
\hline 50 & 8123606 & MGC39372 \\
\hline 51 & 8152215 & KLF10 \\
\hline 52 & 8158147 & SLC25A25 \\
\hline 53 & 8164336 & DPM2 \\
\hline \multicolumn{3}{|c|}{ Cluster 25} \\
\hline 1 & 7904361 & FAM46C \\
\hline 2 & 7906622 & LY9 \\
\hline 3 & 7956287 & NAB2 \\
\hline 4 & 7965040 & PHLDA1 \\
\hline 5 & 7985493 & TM6SF1 \\
\hline 6 & 8044766 & INSIG2 \\
\hline 7 & 8082086 & PARP15 \\
\hline 8 & 8100231 & TEC \\
\hline 9 & 8102800 & SLC7A11 \\
\hline 10 & 8113073 & ARRDC3 \\
\hline 11 & 8118142 & TNF \\
\hline 12 & 8122265 & TNFAIP3 \\
\hline 13 & 8122334 & CCRL1 \\
\hline 14 & 8128123 & RRAGD \\
\hline 15 & 8129677 & SGK1 \\
\hline 16 & 8131844 & GPNMB \\
\hline 17 & 8151816 & GEM \\
\hline 18 & 8177983 & TNF \\
\hline 19 & 8179263 & TNF \\
\hline \multicolumn{3}{|c|}{ Cluster 26} \\
\hline 1 & 7906613 & SLAMF7 \\
\hline \multicolumn{3}{|c|}{ Cluster 27} \\
\hline 1 & 7904726 & TXNIP \\
\hline 2 & 7909214 & RASSF5 \\
\hline 3 & 7921625 & SLAMF6 \\
\hline 4 & 7922474 & KIAA0040 \\
\hline
\end{tabular}




\begin{tabular}{|l|l|l|}
\hline $\mathbf{5}$ & 7930139 & TRIM8 \\
\hline $\mathbf{6}$ & 7931863 & ASB13 \\
\hline $\mathbf{7}$ & 7947624 & PHF21A \\
\hline $\mathbf{8}$ & 7955589 & NR4A1 \\
\hline $\mathbf{9}$ & 7963575 & EIF4B \\
\hline $\mathbf{1 0}$ & 7966183 & ALKBH2 \\
\hline $\mathbf{1 1}$ & 7969677 & MBNL2 \\
\hline $\mathbf{1 2}$ & 7975626 & C14orf43 \\
\hline $\mathbf{1 3}$ & 7978644 & NFKBIA \\
\hline $\mathbf{1 4}$ & 7988212 & ELL3 \\
\hline $\mathbf{1 5}$ & 7996571 & FAM65A \\
\hline $\mathbf{1 6}$ & 8008263 & PDK2 \\
\hline $\mathbf{1 7}$ & 8028652 & ZFP36 \\
\hline $\mathbf{1 8}$ & 8029693 & FOSB \\
\hline $\mathbf{1 9}$ & 8042811 & TET3 \\
\hline
\end{tabular}

\begin{tabular}{|l|l|l|}
\hline $\mathbf{2 0}$ & 8075673 & RBFOX2 \\
\hline $\mathbf{2 1}$ & 8084880 & HES1 \\
\hline $\mathbf{2 2}$ & 8088142 & CHDH \\
\hline $\mathbf{2 3}$ & 8088180 & WNT5A \\
\hline $\mathbf{2 4}$ & 8094743 & RHOH \\
\hline $\mathbf{2 5}$ & 8108370 & EGR1 \\
\hline $\mathbf{2 6}$ & 8116910 & HIVEP1 \\
\hline $\mathbf{2 7}$ & 8117106 & RNF144B \\
\hline $\mathbf{2 8}$ & 8119161 & PIM1 \\
\hline $\mathbf{2 9}$ & 8121850 & HEY2 \\
\hline $\mathbf{3 0}$ & 8132819 & IKZF1 \\
\hline $\mathbf{3 1}$ & 8149720 & EGR3 \\
\hline $\mathbf{3 2}$ & 8151101 & MYBL1 \\
\hline $\mathbf{3 3}$ & 8163629 & TNFSF8 \\
\hline $\mathbf{3 4}$ & 8165496 & TUBB4B \\
\hline
\end{tabular}



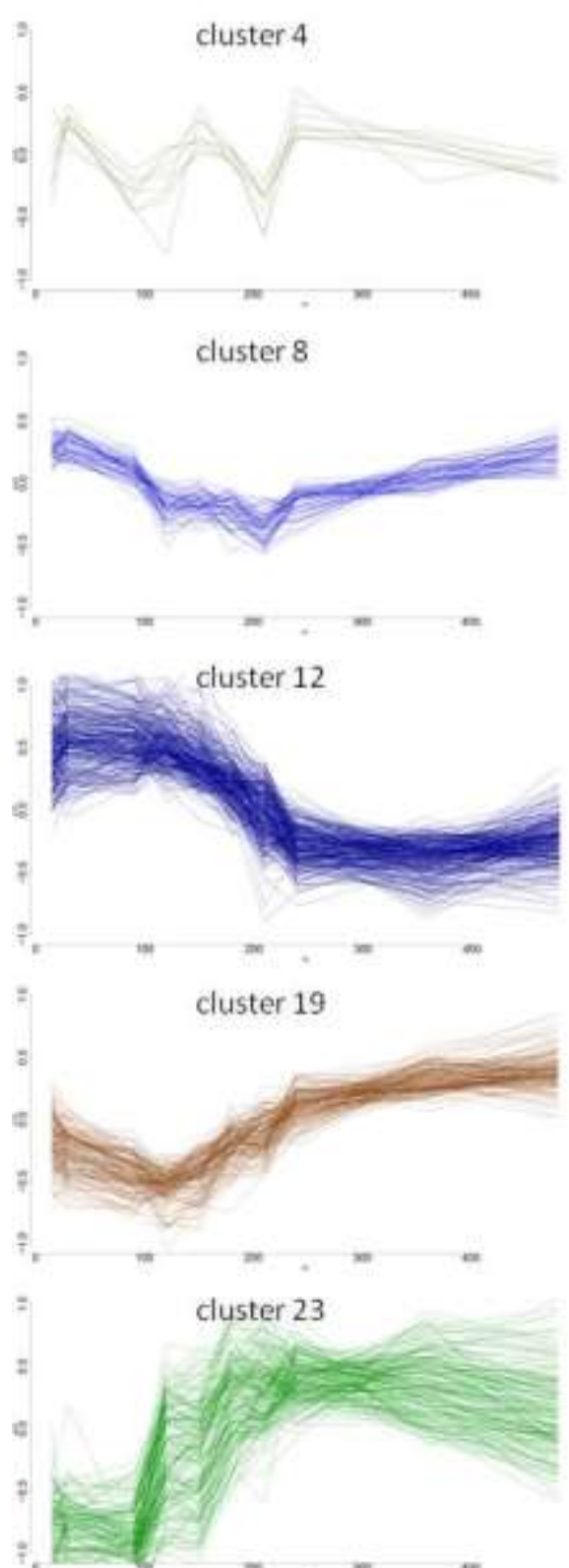

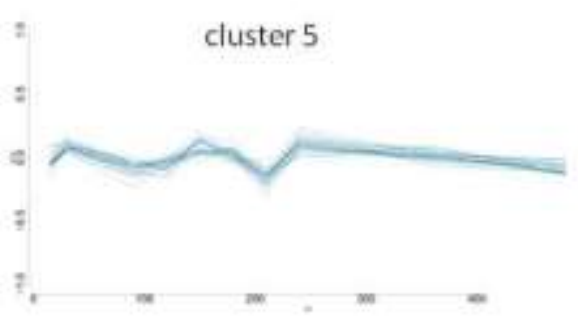

cluster 9
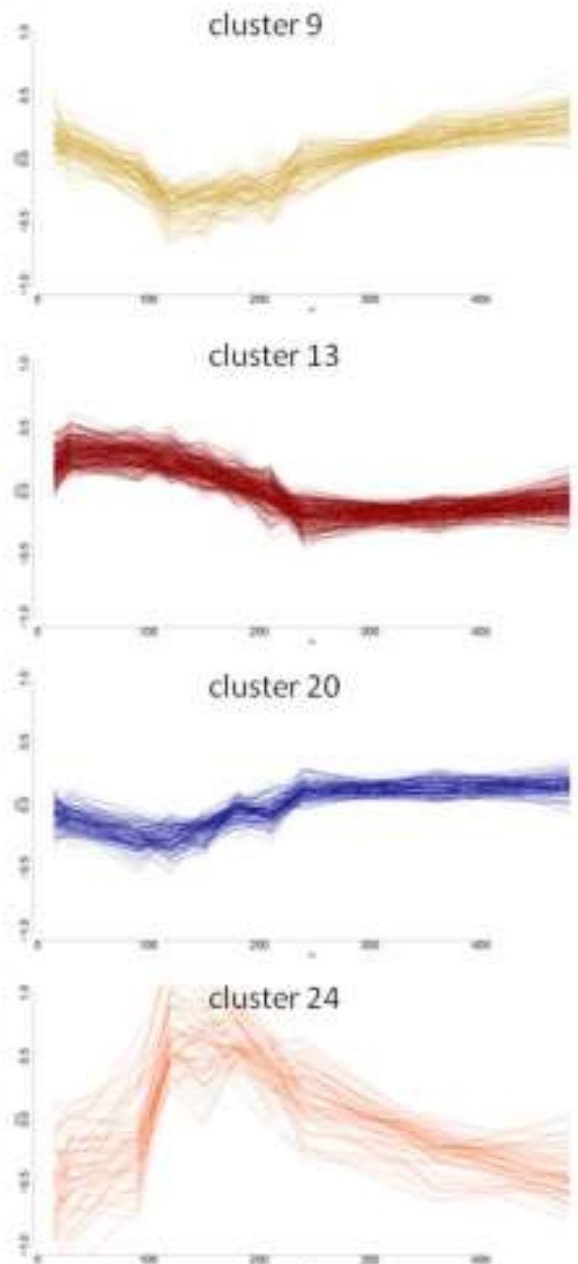

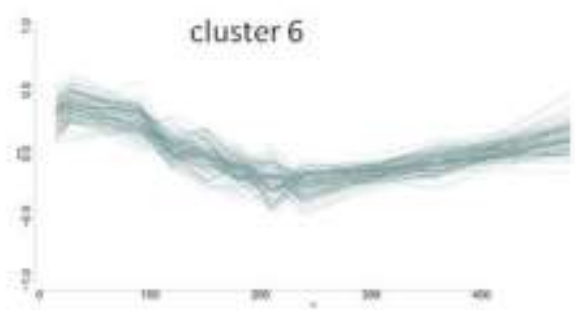

cluster 10

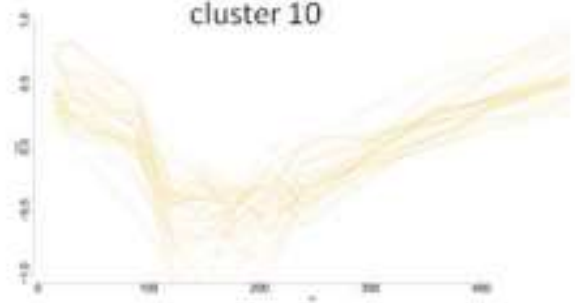

cluster 17

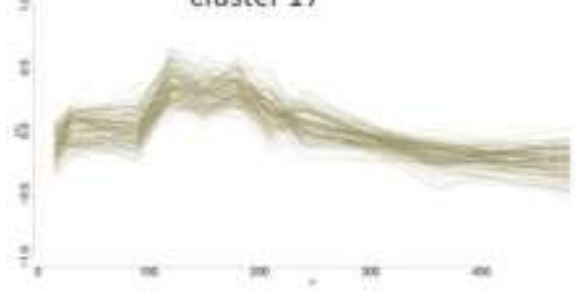

cluster 21
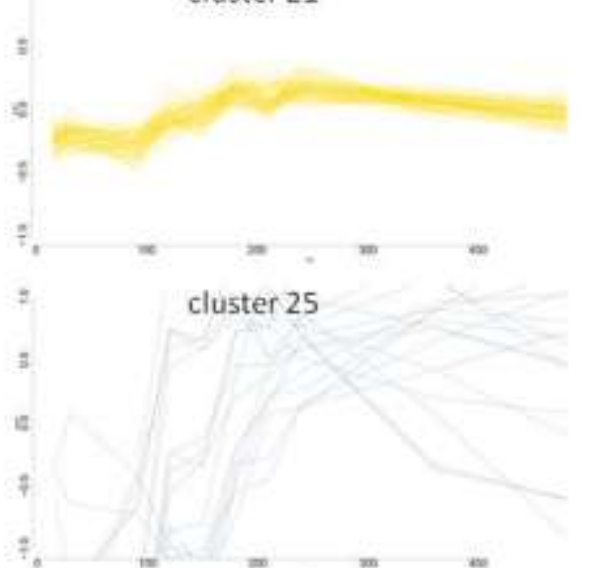

cluster 7

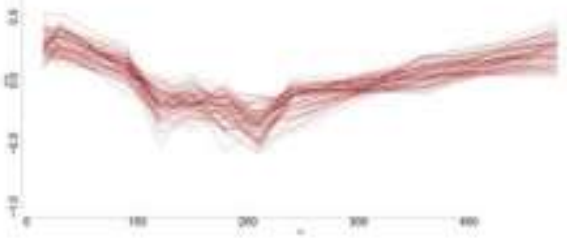

cluster 11

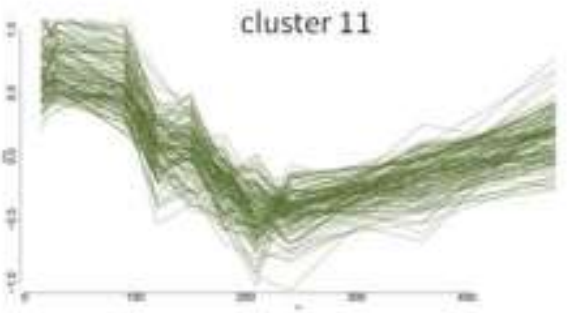

cluster 18

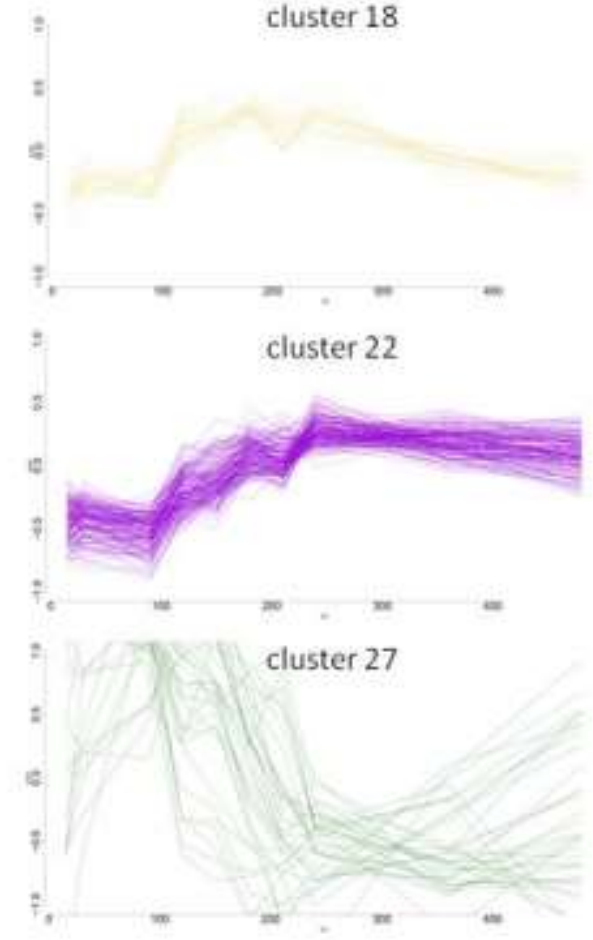

Figure A-1 (see next page for legend) 
Figure A-1 Bayesian Hierarchical Clustering reveals 27 clusters of genes bearing differential expression over time. $\alpha$-IgM stimulation of BL2 cells was monitored with regard to gene expression changes on the whole genome level for a period of 8 hours. Three biological replicates were hybridized to Human Gene ST 1.0 microarray chips (Affymetrix)

Bayesian hierarchical clustering identified 21 clusters of genes with an expression range $>0.5$. Clusters of genes with less than $10 \%$ significantly enriched genes (FDR $<0.05$ ) were excluded (cluster 26 contains only SLAMF7). Depicted are the time courses of each gene in one cluster. 
Table A-2 Gene set enrichment analysis for manually picked clusters (cl 6, cl 8 and $\mathrm{cl} 11$ )

\begin{tabular}{|c|c|c|c|c|c|c|}
\hline GOBPID & Pvalue & OddsRatio & ExpCount & Count & Size & Term \\
\hline \multicolumn{7}{|l|}{ cluster 6} \\
\hline GO:0044267 & 0.000 & 2.544 & 16 & 30 & 2926 & $\begin{array}{l}\text { cellular protein metabolic } \\
\text { process }\end{array}$ \\
\hline GO:0043153 & 0.000 & 191.784 & 0 & 2 & 4 & $\begin{array}{l}\text { entrainment of circadian clock by } \\
\text { photoperiod }\end{array}$ \\
\hline GO:0006464 & 0.000 & 2.492 & 12 & 24 & 2242 & protein modification process \\
\hline GO:0009648 & 0.000 & 95.878 & 0 & 2 & 6 & photoperiodism \\
\hline GO:0033235 & 0.001 & 76.697 & 0 & 2 & 7 & $\begin{array}{l}\text { positive regulation of protein } \\
\text { sumoylation }\end{array}$ \\
\hline GO:0043412 & 0.001 & 2.373 & 12 & 24 & 2335 & macromolecule modification \\
\hline GO:0033233 & 0.001 & 63.910 & 0 & 2 & 8 & $\begin{array}{l}\text { regulation of protein } \\
\text { sumoylation }\end{array}$ \\
\hline GO:0043501 & 0.001 & 63.910 & 0 & 2 & 8 & skeletal muscle adaptation \\
\hline GO:0009649 & 0.001 & 54.776 & 0 & 2 & 9 & entrainment of circadian clock \\
\hline GO:0019538 & 0.001 & 2.153 & 18 & 31 & 3472 & protein metabolic process \\
\hline GO:0071397 & 0.001 & 47.926 & 0 & 2 & 10 & cellular response to cholesterol \\
\hline GO:0014888 & 0.002 & 38.335 & 0 & 2 & 12 & striated muscle adaptation \\
\hline GO:0006486 & 0.004 & 5.136 & 1 & 5 & 197 & protein glycosylation \\
\hline GO:0043413 & 0.004 & 5.136 & 1 & 5 & 197 & macromolecule glycosylation \\
\hline GO:0070085 & 0.004 & 5.082 & 1 & 5 & 199 & glycosylation \\
\hline GO:0050691 & 0.005 & 22.539 & 0 & 2 & 19 & $\begin{array}{l}\text { regulation of defense response } \\
\text { to virus by host }\end{array}$ \\
\hline GO:0070723 & 0.005 & 22.539 & 0 & 2 & 19 & response to cholesterol \\
\hline GO:0044260 & 0.005 & 1.858 & 32 & 44 & 6081 & $\begin{array}{l}\text { cellular macromolecule } \\
\text { metabolic process }\end{array}$ \\
\hline GO:0006507 & 0.005 & Inf & 0 & 1 & 1 & GPI anchor release \\
\hline GO:0046092 & 0.005 & Inf & 0 & 1 & 1 & deoxycytidine metabolic process \\
\hline GO:0048213 & 0.005 & Inf & 0 & 1 & 1 & $\begin{array}{l}\text { Golgi vesicle prefusion complex } \\
\text { stabilization }\end{array}$ \\
\hline GO:0051754 & 0.005 & Inf & 0 & 1 & 1 & $\begin{array}{l}\text { meiotic sister chromatid } \\
\text { cohesion, centromeric }\end{array}$ \\
\hline GO:0061310 & 0.005 & Inf & 0 & 1 & 1 & $\begin{array}{l}\text { canonical Wnt receptor signaling } \\
\text { pathway involved in cardiac } \\
\text { neural crest cell differentiation } \\
\text { involved in heart development }\end{array}$ \\
\hline GO:0070159 & 0.005 & Inf & 0 & 1 & 1 & $\begin{array}{l}\text { mitochondrial threonyl-tRNA } \\
\text { aminoacylation }\end{array}$ \\
\hline GO:0071401 & 0.005 & Inf & 0 & 1 & 1 & cellular response to triglyceride \\
\hline GO:0071609 & 0.005 & Inf & 0 & 1 & 1 & $\begin{array}{l}\text { chemokine ( } \mathrm{C}-\mathrm{C} \text { motif) ligand } 5 \\
\text { production }\end{array}$ \\
\hline GO:0071612 & 0.005 & Inf & 0 & 1 & 1 & IP-10 production \\
\hline GO:0071649 & 0.005 & Inf & 0 & 1 & 1 & $\begin{array}{l}\text { regulation of chemokine (C-C } \\
\text { motif) ligand } 5 \text { production }\end{array}$ \\
\hline GO:0071651 & 0.005 & Inf & 0 & 1 & 1 & $\begin{array}{l}\text { positive regulation of chemokine } \\
\text { (C-C motif) ligand } 5 \text { production }\end{array}$ \\
\hline GO:0071658 & 0.005 & Inf & 0 & 1 & 1 & regulation of IP-10 production \\
\hline GO:0071660 & 0.005 & Inf & 0 & 1 & 1 & $\begin{array}{l}\text { positive regulation of IP-10 } \\
\text { production }\end{array}$ \\
\hline
\end{tabular}




\begin{tabular}{|c|c|c|c|c|c|c|}
\hline GO:0090219 & 0.005 & Inf & 0 & 1 & 1 & $\begin{array}{l}\text { negative regulation of lipid } \\
\text { kinase activity }\end{array}$ \\
\hline GO:0097241 & 0.005 & $\operatorname{lnf}$ & 0 & 1 & 1 & $\begin{array}{l}\text { hematopoietic stem cell } \\
\text { migration to bone marrow }\end{array}$ \\
\hline GO:1900037 & 0.005 & $\operatorname{lnf}$ & 0 & 1 & 1 & $\begin{array}{l}\text { regulation of cellular response to } \\
\text { hypoxia }\end{array}$ \\
\hline GO:2000055 & 0.005 & $\operatorname{lnf}$ & 0 & 1 & 1 & $\begin{array}{l}\text { positive regulation of Wnt } \\
\text { receptor signaling pathway } \\
\text { involved in dorsal/ventral axis } \\
\text { specification }\end{array}$ \\
\hline GO:2000148 & 0.005 & $\operatorname{lnf}$ & 0 & 1 & 1 & $\begin{array}{l}\text { regulation of planar cell polarity } \\
\text { pathway involved in ventricular } \\
\text { septum morphogenesis }\end{array}$ \\
\hline GO:2000149 & 0.005 & $\operatorname{lnf}$ & 0 & 1 & 1 & $\begin{array}{l}\text { negative regulation of planar cell } \\
\text { polarity pathway involved in } \\
\text { ventricular septum } \\
\text { morphogenesis }\end{array}$ \\
\hline GO:2000150 & 0.005 & $\operatorname{lnf}$ & 0 & 1 & 1 & $\begin{array}{l}\text { regulation of planar cell polarity } \\
\text { pathway involved in cardiac } \\
\text { muscle tissue morphogenesis }\end{array}$ \\
\hline GO:2000151 & 0.005 & $\operatorname{lnf}$ & 0 & 1 & 1 & $\begin{array}{l}\text { negative regulation of planar cell } \\
\text { polarity pathway involved in } \\
\text { cardiac muscle tissue } \\
\text { morphogenesis }\end{array}$ \\
\hline GO:2000159 & 0.005 & $\operatorname{lnf}$ & 0 & 1 & 1 & $\begin{array}{l}\text { regulation of planar cell polarity } \\
\text { pathway involved in heart } \\
\text { morphogenesis }\end{array}$ \\
\hline GO:2000160 & 0.005 & $\operatorname{lnf}$ & 0 & 1 & 1 & $\begin{array}{l}\text { negative regulation of planar cell } \\
\text { polarity pathway involved in } \\
\text { heart morphogenesis }\end{array}$ \\
\hline GO:2000161 & 0.005 & $\operatorname{lnf}$ & 0 & 1 & 1 & $\begin{array}{l}\text { regulation of planar cell polarity } \\
\text { pathway involved in cardiac right } \\
\text { atrium morphogenesis }\end{array}$ \\
\hline GO:2000162 & 0.005 & $\operatorname{lnf}$ & 0 & 1 & 1 & $\begin{array}{l}\text { negative regulation of planar cell } \\
\text { polarity pathway involved in } \\
\text { cardiac right atrium } \\
\text { morphogenesis }\end{array}$ \\
\hline GO:2000163 & 0.005 & $\operatorname{lnf}$ & 0 & 1 & 1 & $\begin{array}{l}\text { regulation of planar cell polarity } \\
\text { pathway involved in outflow } \\
\text { tract morphogenesis }\end{array}$ \\
\hline GO:2000164 & 0.005 & $\operatorname{lnf}$ & 0 & 1 & 1 & $\begin{array}{l}\text { negative regulation of planar cell } \\
\text { polarity pathway involved in } \\
\text { outflow tract morphogenesis }\end{array}$ \\
\hline GO:2000165 & 0.005 & $\operatorname{lnf}$ & 0 & 1 & 1 & $\begin{array}{l}\text { regulation of planar cell polarity } \\
\text { pathway involved in pericardium } \\
\text { morphogenesis }\end{array}$ \\
\hline GO:2000166 & 0.005 & $\operatorname{lnf}$ & 0 & 1 & 1 & $\begin{array}{l}\text { negative regulation of planar cell } \\
\text { polarity pathway involved in } \\
\text { pericardium morphogenesis }\end{array}$ \\
\hline GO:2000167 & 0.005 & $\operatorname{lnf}$ & 0 & 1 & 1 & $\begin{array}{l}\text { regulation of planar cell polarity } \\
\text { pathway involved in neural tube } \\
\text { closure }\end{array}$ \\
\hline
\end{tabular}




\begin{tabular}{|c|c|c|c|c|c|c|}
\hline GO:2000168 & 0.005 & Inf & 0 & 1 & 1 & $\begin{array}{l}\text { negative regulation of planar cell } \\
\text { polarity pathway involved in } \\
\text { neural tube closure }\end{array}$ \\
\hline GO:0016925 & 0.006 & 20.164 & 0 & 2 & 21 & protein sumoylation \\
\hline GO:0070887 & 0.006 & 2.306 & 7 & 15 & 1383 & $\begin{array}{l}\text { cellular response to chemical } \\
\text { stimulus }\end{array}$ \\
\hline GO:0071310 & 0.007 & 2.466 & 5 & 12 & 1015 & $\begin{array}{l}\text { cellular response to organic } \\
\text { substance }\end{array}$ \\
\hline GO:0006890 & 0.007 & 17.410 & 0 & 2 & 24 & $\begin{array}{l}\text { retrograde vesicle-mediated } \\
\text { transport, Golgi to ER }\end{array}$ \\
\hline GO:0044092 & 0.007 & 2.849 & 3 & 9 & 648 & $\begin{array}{l}\text { negative regulation of molecular } \\
\text { function }\end{array}$ \\
\hline GO:0019835 & 0.008 & 15.957 & 0 & 2 & 26 & cytolysis \\
\hline GO:0071396 & 0.009 & 15.318 & 0 & 2 & 27 & cellular response to lipid \\
\hline GO:0010907 & 0.010 & 14.728 & 0 & 2 & 28 & $\begin{array}{l}\text { positive regulation of glucose } \\
\text { metabolic process }\end{array}$ \\
\hline GO:0034381 & 0.010 & 14.728 & 0 & 2 & 28 & $\begin{array}{l}\text { plasma lipoprotein particle } \\
\text { clearance }\end{array}$ \\
\hline GO:0009101 & 0.010 & 4.094 & 1 & 5 & 245 & $\begin{array}{l}\text { glycoprotein biosynthetic } \\
\text { process }\end{array}$ \\
\hline
\end{tabular}

Table A-3 List of genes, taken for comparison of CD40 and BCR stimulated BL cells by testing for stationarity.

\begin{tabular}{|l|l|l|l|l|}
\hline & Gene ID & Probe ID & Gene Symbol & Gene Name \\
\hline $\mathbf{1}$ & 790 & 7906613 & SLAMF7 & SLAM family member 7 \\
\hline $\mathbf{1}$ & 587 & 7904361 & FAM46C & family with sequence similarity 46, member C \\
\hline $\mathbf{1}$ & 762 & 7906339 & CD1A & CD1a molecule \\
\hline $\mathbf{1}$ & 763 & 7906348 & CD1C & CD1c molecule \\
\hline $\mathbf{1}$ & 1169 & 7911754 & TNFRSF14 & tumor necrosis factor receptor superfamily, member \\
\hline $\mathbf{1}$ & 1216 & 7912496 & MTHFR & methylenetetrahydrofolate reductase (NAD(P)H) \\
\hline $\mathbf{1}$ & 2072 & 7923917 & FAIM3 & Fas apoptotic inhibitory molecule 3 \\
\hline $\mathbf{1}$ & 2548 & 7930413 & DUSP5 & dual specificity phosphatase 5 \\
\hline $\mathbf{1}$ & 5066 & 7961891 & BHLHE41 & basic helix-loop-helix family, member e41 \\
\hline $\mathbf{1}$ & 5207 & 7963911 & CD63 & CD63 molecule \\
\hline $\mathbf{1}$ & 5286 & 7965040 & PHLDA1 & pleckstrin homology-like domain, family A, member \\
\hline $\mathbf{1}$ & 5790 & 7972557 & GPR183 & G protein-coupled receptor 183 \\
\hline $\mathbf{1}$ & 7130 & 7990345 & SEMA7A & semaphorin 7A, GPI membrane anchor (John Milton Ha \\
\hline $\mathbf{1}$ & 7327 & 7992789 & TNFRSF12A & tumor necrosis factor receptor superfamily, member \\
\hline $\mathbf{1}$ & 8388 & 8006608 & CCL4L1 & chemokine (C-C motif) ligand 4-like 1 \\
\hline $\mathbf{1}$ & 8389 & 8006621 & CCL4L1 & chemokine (C-C motif) ligand 4-like 1 \\
\hline $\mathbf{1}$ & 8623 & 8009476 & MAP2K6 & mitogen-activated protein kinase kinase 6 \\
\hline $\mathbf{1}$ & 9420 & 8019651 & CCL4L1 & chemokine (C-C motif) ligand 4-like 1 \\
\hline $\mathbf{1}$ & 10263 & 8030007 & EMP3 & epithelial membrane protein 3 \\
\hline $\mathbf{1}$ & 12534 & 8059854 & ARL4C & ADP-ribosylation factor-like 4C \\
\hline $\mathbf{1}$ & 13836 & 8075886 & IL2RB & interleukin 2 receptor, beta \\
\hline $\mathbf{1}$ & 14768 & 8088848 & PDZRN3 & PDZ domain containing ring finger 3 \\
\hline $\mathbf{1}$ & 15587 & 8100231 & TEC & tec protein tyrosine kinase \\
\hline $\mathbf{1}$ & 17463 & 8125139 & NEU1 & sialidase 1 (lysosomal sialidase) \\
\hline $\mathbf{1}$ & 18005 & 8132725 & UPP1 & uridine phosphorylase 1 \\
\hline $\mathbf{1}$ & 19132 & 8147344 & PDP1 & pyruvate dehyrogenase phosphatase catalytic subuni \\
\hline & & & & \\
\hline
\end{tabular}




\begin{tabular}{|c|c|c|c|c|}
\hline 1 & 19304 & 8149330 & CTSB & cathepsin B \\
\hline 1 & 19510 & 8151816 & GEM & GTP binding protein overexpressed in skeletal musc \\
\hline 1 & 21675 & 8178676 & NEU1 & sialidase 1 (lysosomal sialidase) \\
\hline 1 & 21798 & 8179851 & NEU1 & sialidase 1 (lysosomal sialidase) \\
\hline 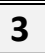 & 13173 & 8068022 & MIR155HG & MIR155 host gene (non-protein coding) \\
\hline 3 & 1056 & 7910427 & GALNT2 & UDP-N-acetyl-alpha-D-galactosamine:polypeptide $\mathrm{N}-\mathrm{a}$ \\
\hline 3 & 1073 & 7910680 & GPR137B & G protein-coupled receptor 137B \\
\hline 3 & 1347 & 7914202 & SNHG12 & small nucleolar RNA host gene 12 (non-protein codi \\
\hline 3 & 1348 & 7914212 & SNORA61 & small nucleolar RNA, H/ACA box 61 \\
\hline 3 & 1601 & 7917754 & BCAR3 & breast cancer anti-estrogen resistance 3 \\
\hline 3 & 1677 & 7918902 & CD58 & CD58 molecule \\
\hline 3 & 5457 & 7967624 & SLC15A4 & solute carrier family 15 , member 4 \\
\hline 3 & 6922 & 7986665 & NIPA2 & non imprinted in Prader-Willi/Angelman syndrome 2 \\
\hline 3 & 7106 & 7989968 & CALML4 & calmodulin-like 4 \\
\hline 3 & 9034 & 8015031 & CCR7 & chemokine ( $\mathrm{C}-\mathrm{C}$ motif) receptor 7 \\
\hline 3 & 9716 & 8023646 & $\mathrm{BCL2}$ & B-cell CLL/lymphoma 2 \\
\hline 3 & 9922 & 8026007 & ZNF791 & zinc finger protein 791 \\
\hline 3 & 10765 & 8035694 & PBX4 & pre-B-cell leukemia homeobox 4 \\
\hline 3 & 12770 & 8062964 & SYS1 & SYS1 Golgi-localized integral membrane protein hom \\
\hline 3 & 13776 & 8075130 & PITPNB & phosphatidylinositol transfer protein, beta \\
\hline 3 & 13965 & 8077450 & ARL8B & ADP-ribosylation factor-like 8B \\
\hline 3 & 14296 & 8082086 & PARP15 & poly (ADP-ribose) polymerase family, member 15 \\
\hline 3 & 15864 & 8103951 & ACSL1 & acyl-CoA synthetase long-chain family member 1 \\
\hline 3 & 15939 & 8105067 & PTGER4 & prostaglandin E receptor 4 (subtype EP4) \\
\hline 3 & 16336 & 8110569 & SQSTM1 & sequestosome 1 \\
\hline 3 & 16365 & 8110886 & MED10 & mediator complex subunit 10 \\
\hline 3 & 16424 & 8111941 & HMGCS1 & 3-hydroxy-3-methylglutaryl-CoA synthase 1 (soluble \\
\hline 3 & 16530 & 8113305 & CHD1 & chromodomain helicase DNA binding protein 1 \\
\hline 3 & 16741 & 8116227 & CLK4 & CDC-like kinase 4 \\
\hline 3 & 17286 & 8123181 & IGF2R & insulin-like growth factor 2 receptor \\
\hline 3 & 17482 & 8125447 & HLA-DQB1 & major histocompatibility complex, class II, DQ bet \\
\hline 3 & 17583 & 8126666 & NFKBIE & nuclear factor of kappa light polypeptide gene enh \\
\hline 3 & 19015 & 8145691 & UBXN8 & UBX domain protein 8 \\
\hline 3 & 21736 & 8179258 & LTA & lymphotoxin alpha (TNF superfamily, member 1) \\
\hline 4 & 9890 & 8025601 & ICAM1 & "intercellular adhesion molecule 1 \\
\hline 4 & 287 & 7900146 & ZC3H12A & zinc finger $\mathrm{CCCH}$-type containing $12 \mathrm{~A}$ \\
\hline 4 & 606 & 7904702 & NOTCH2NL & notch $2 \mathrm{~N}$-terminal like \\
\hline 4 & 987 & 7909503 & SERTAD4 & SERTA domain containing 4 \\
\hline 4 & 1697 & 7919193 & NUDT4P1 & nudix (nucleoside diphosphate linked moiety X)-typ \\
\hline 4 & 2071 & 7923907 & IL10 & interleukin 10 \\
\hline 4 & 2450 & 7929032 & FAS & Fas (TNF receptor superfamily, member 6) \\
\hline 4 & 2525 & 7930074 & NFKB2 & nuclear factor of kappa light polypeptide gene enh \\
\hline 4 & 2778 & 7933750 & SLC16A9 & solute carrier family 16, member 9 (monocarboxylic \\
\hline 4 & 6047 & 7975793 & BATF & basic leucine zipper transcription factor, ATF-lik \\
\hline 4 & 6149 & 7977018 & TRAF3 & TNF receptor-associated factor 3 \\
\hline 4 & 6493 & 7981530 & GPR132 & G protein-coupled receptor 132 \\
\hline 4 & 6946 & 7987048 & MTMR10 & myotubularin related protein 10 \\
\hline 4 & 7097 & 7989849 & DENND4A & DENN/MADD domain containing 4A \\
\hline 4 & 7833 & 7999496 & ZC3H7A & zinc finger $\mathrm{CCCH}$-type containing $7 \mathrm{~A}$ \\
\hline 4 & 8038 & 8002152 & SLC12A4 & solute carrier family 12 (potassium/chloride trans \\
\hline
\end{tabular}




\begin{tabular}{|c|c|c|c|c|}
\hline 4 & 8523 & 8008096 & SNX11 & sorting nexin 11 \\
\hline 4 & 9345 & 8018922 & CYTH1 & cytohesin 1 \\
\hline 4 & 9790 & 8024485 & GADD45B & growth arrest and DNA-damage-inducible, beta \\
\hline 4 & 11145 & 8039545 & NLRP11 & NLR family, pyrin domain containing 11 \\
\hline 4 & 11467 & 8043583 & LOC285033 & hypothetical protein LOC285033 \\
\hline 4 & 12722 & 8062319 & TGIF2 & TGFB-induced factor homeobox 2 \\
\hline 4 & 13294 & 8069541 & SAMSN1 & SAM domain, SH3 domain and nuclear localization si \\
\hline 4 & 14827 & 8089771 & CD80 & CD80 molecule \\
\hline 4 & 15907 & 8104580 & FAM105B & family with sequence similarity 105 , member $B$ \\
\hline 4 & 15908 & 8104590 & LOC100130744 & hypothetical LOC100130744 \\
\hline 4 & 16139 & 8108080 & PHF15 & PHD finger protein 15 \\
\hline 4 & 17270 & 8122933 & TIAM2 & T-cell lymphoma invasion and metastasis 2 \\
\hline 4 & 18253 & 8136067 & TSPAN33 & tetraspanin 33 \\
\hline 4 & 20028 & 8158918 & GTF3C4 & general transcription factor IIIC, polypeptide 4, \\
\hline 5 & 16514 & 8113073 & ARRDC3 & arrestin domain containing 3 \\
\hline 5 & 908 & 7908409 & RGS2 & regulator of G-protein signaling 2, 24kDa \\
\hline 5 & 2158 & 7925062 & SIPA1L2 & signal-induced proliferation-associated 1 like 2 \\
\hline 5 & 4441 & 7953532 & ENO2 & enolase 2 (gamma, neuronal) \\
\hline 5 & 4992 & 7961075 & CD69 & CD69 molecule \\
\hline 5 & 8963 & 8014063 & $\mathrm{EV} I 2 \mathrm{~B}$ & ecotropic viral integration site $2 \mathrm{~B}$ \\
\hline 5 & 8964 & 8014066 & $\mathrm{EV} I 2 \mathrm{~A}$ & ecotropic viral integration site $2 \mathrm{~A}$ \\
\hline 5 & 12671 & 8061564 & ID1 & inhibitor of DNA binding 1, dominant negative heli \\
\hline 5 & 14645 & 8086961 & PFKFB4 & 6-phosphofructo-2-kinase/fructose-2,6-biphosphatas \\
\hline 5 & 15286 & 8095870 & CCNG2 & cyclin G2 \\
\hline 5 & 15296 & 8096070 & BMP3 & bone morphogenetic protein 3 \\
\hline 5 & 15668 & 8101322 & MOP-1 & MOP-1 \\
\hline 5 & 16475 & 8112615 & ENC1 & ectodermal-neural cortex 1 (with BTB-like domain) \\
\hline 5 & 16810 & 8117020 & MYLIP & myosin regulatory light chain interacting protein \\
\hline 5 & 17049 & 8119898 & VEGFA & vascular endothelial growth factor $A$ \\
\hline 5 & 17197 & 8121861 & NCOA7 & nuclear receptor coactivator 7 \\
\hline 5 & 17371 & 8124380 & HIST1H1A & histone cluster $1, \mathrm{H} 1 \mathrm{a}$ \\
\hline 5 & 17884 & 8131069 & GPER & G protein-coupled estrogen receptor 1 \\
\hline 5 & 19666 & 8154100 & VLDLR & very low density lipoprotein receptor \\
\hline 6 & 16808 & 8116983 & CD83 & CD83 molecule \\
\hline 6 & 1992 & 7922717 & RGS16 & regulator of G-protein signaling 16 \\
\hline 6 & 2110 & 7924450 & DUSP10 & dual specificity phosphatase 10 \\
\hline 6 & 2654 & 7931914 & IL2RA & interleukin 2 receptor, alpha \\
\hline 6 & 3136 & 7938329 & SNORA23 & small nucleolar RNA, H/ACA box 23 \\
\hline 6 & 3578 & 7943424 & BIRC2 & baculoviral IAP repeat-containing 2 \\
\hline 6 & 4202 & 7950743 & RAB30 & RAB30, member RAS oncogene family \\
\hline 6 & 4515 & 7954436 & LRMP & lymphoid-restricted membrane protein \\
\hline 6 & 6013 & 7975368 & SRSF5 & serine/arginine-rich splicing factor 5 \\
\hline 6 & 6751 & 7984364 & SMAD3 & SMAD family member 3 \\
\hline 6 & 7846 & 7999642 & KIAA0430 & KIAA0430 \\
\hline 6 & 10766 & 8035703 & LPAR2 & lysophosphatidic acid receptor 2 \\
\hline 6 & 10812 & 8036207 & NFKBID & nuclear factor of kappa light polypeptide gene enh \\
\hline 6 & 12050 & 8052269 & CCDC88A & coiled-coil domain containing $88 \mathrm{~A}$ \\
\hline 6 & 12786 & 8063156 & CD40 & CD40 molecule, TNF receptor superfamily member 5 \\
\hline 6 & 12796 & 8063351 & SLC9A8 & solute carrier family 9 (sodium/hydrogen exchanger \\
\hline 6 & 13225 & 8068551 & DYRK1A & dual-specificity tyrosine-(Y)-phosphorylation regu \\
\hline
\end{tabular}




\begin{tabular}{|c|c|c|c|c|}
\hline 6 & 14291 & 8082035 & CD86 & CD86 molecule \\
\hline 6 & 14449 & 8084219 & KLHL24 & kelch-like 24 (Drosophila) \\
\hline 6 & 14763 & 8088776 & FOXP1 & forkhead box P1 \\
\hline 6 & 14942 & 8091485 & $\mathrm{SIAH} 2$ & seven in absentia homolog 2 (Drosophila) \\
\hline 6 & 15555 & 8099760 & ARAP2 & ArfGAP with RhoGAP domain, ankyrin repeat and PH d \\
\hline 6 & 16561 & 8113761 & ZNF608 & zinc finger protein 608 \\
\hline 6 & 17168 & 8121563 & MARCKS & myristoylated alanine-rich protein kinase $\mathrm{C}$ substr \\
\hline 6 & 17232 & 8122343 & HECA & headcase homolog (Drosophila) \\
\hline 6 & 17315 & 8123606 & MGC39372 & serpin peptidase inhibitor, clade B (ovalbumin), $\mathrm{m}$ \\
\hline 6 & 17347 & 8123981 & C6orf114 & chromosome 6 open reading frame 114 \\
\hline 6 & 18983 & 8145136 & PPP3CC & protein phosphatase 3 , catalytic subunit, gamma is \\
\hline 6 & 19386 & 8150225 & RAB11FIP1 & RAB11 family interacting protein 1 (class I) \\
\hline 6 & 21458 & 8176384 & ZFY & zinc finger protein, $\mathrm{Y}$-linked \\
\hline 8 & 14277 & 8081838 & ARHGAP31 & Rho GTPase activating protein 31 \\
\hline 8 & 785 & 7906564 & PEA15 & phosphoprotein enriched in astrocytes 15 \\
\hline 8 & 1956 & 7922343 & TNFSF4 & tumor necrosis factor (ligand) superfamily, member \\
\hline 8 & 2819 & 7934367 & ANXA7 & annexin $\mathrm{A} 7$ \\
\hline 8 & 2875 & 7935146 & NOC3L & nucleolar complex associated 3 homolog (S. cerevis \\
\hline 8 & 4468 & 7953873 & OVOS & ovostatin \\
\hline 8 & 4988 & 7961026 & OVOS & ovostatin \\
\hline 8 & 5147 & 7963235 & CSRNP2 & cysteine-serine-rich nuclear protein 2 \\
\hline 8 & 5377 & 7966356 & HVCN1 & hydrogen voltage-gated channel 1 \\
\hline 8 & 7363 & 7993185 & NUBP1 & nucleotide binding protein 1 (MinD homolog, E. col \\
\hline 8 & 7968 & 8001185 & DNAJA2 & DnaJ (Hsp40) homolog, subfamily A, member 2 \\
\hline 8 & 8679 & 8010243 & SYNGR2 & synaptogyrin 2 \\
\hline 8 & 8735 & 8011027 & MYO1C & myosin IC \\
\hline 8 & 9145 & 8016194 & MAP3K14 & mitogen-activated protein kinase kinase kinase 14 \\
\hline 8 & 9493 & 8020495 & CABLES1 & Cdk5 and Abl enzyme substrate 1 \\
\hline 8 & 9798 & 8024572 & GNA15 & guanine nucleotide binding protein (G protein), al \\
\hline 8 & 11850 & 8049271 & ATG16L1 & ATG16 autophagy related 16-like 1 (S. cerevisiae) \\
\hline 8 & 12201 & 8054192 & MITD1 & MIT, microtubule interacting and transport, domain \\
\hline 8 & 12503 & 8059413 & DOCK10 & dedicator of cytokinesis 10 \\
\hline 8 & 14674 & 8087473 & IP6K1 & inositol hexakisphosphate kinase 1 \\
\hline 8 & 15291 & 8096004 & BMP2K & BMP2 inducible kinase \\
\hline 8 & 16766 & 8116548 & DUSP22 & dual specificity phosphatase 22 \\
\hline 8 & 16908 & 8117890 & HLA-E & major histocompatibility complex, class I, E \\
\hline 8 & 17325 & 8123717 & RPP40 & ribonuclease P/MRP 40kDa subunit \\
\hline 8 & 18070 & 8133459 & CLIP2 & CAP-GLY domain containing linker protein 2 \\
\hline 8 & 20111 & 8159984 & C9orf46 & chromosome 9 open reading frame 46 \\
\hline 8 & 20513 & 8164967 & VAV2 & vav 2 guanine nucleotide exchange factor \\
\hline 8 & 21197 & 8173444 & IL2RG & interleukin 2 receptor, gamma \\
\hline 8 & 21584 & 8177788 & HLA-E & major histocompatibility complex, class I, E \\
\hline 8 & 21721 & 8179103 & HLA-E & major histocompatibility complex, class I, E \\
\hline 9 & 8447 & 8007228 & ATP6V0A1 & ATPase, $\mathrm{H}+$ transporting, lysosomal V0 subunit a1 \\
\hline 9 & 1075 & 7910706 & LGALS8 & lectin, galactoside-binding, soluble, 8 \\
\hline 9 & 3655 & 7944560 & ARHGEF12 & Rho guanine nucleotide exchange factor (GEF) 12 \\
\hline 9 & 4023 & 7948667 & AHNAK & AHNAK nucleoprotein \\
\hline 9 & 4255 & 7951351 & PDGFD & platelet derived growth factor $\mathrm{D}$ \\
\hline 9 & 4444 & 7953569 & PTPN6 & protein tyrosine phosphatase, non-receptor type 6 \\
\hline 9 & 5549 & 7969060 & FNDC3A & fibronectin type III domain containing $3 \mathrm{~A}$ \\
\hline
\end{tabular}




\begin{tabular}{|c|c|c|c|c|}
\hline 9 & 6422 & 7980547 & SEL1L & sel-1 suppressor of lin-12-like (C. elegans) \\
\hline 9 & 6443 & 7980958 & LGMN & legumain \\
\hline 9 & 6474 & 7981290 & WARS & tryptophanyl-tRNA synthetase \\
\hline 9 & 7051 & 7989037 & CCPG1 & cell cycle progression 1 \\
\hline 9 & 8350 & 8006123 & CPD & carboxypeptidase D \\
\hline 9 & 10652 & 8034304 & ACP5 & acid phosphatase 5 , tartrate resistant \\
\hline 9 & 12500 & 8059361 & WDFY1 & WD repeat and FYVE domain containing 1 \\
\hline 9 & 13957 & 8077270 & CHL1 & cell adhesion molecule with homology to L1CAM (clo \\
\hline 9 & 14367 & 8083092 & ZBTB38 & zinc finger and BTB domain containing 38 \\
\hline 9 & 14471 & 8084634 & DNAJB11 & DnaJ (Hsp40) homolog, subfamily B, member 11 \\
\hline 9 & 15358 & 8097017 & UGT8 & UDP glycosyltransferase 8 \\
\hline 9 & 15434 & 8098121 & RAPGEF2 & Rap guanine nucleotide exchange factor (GEF) 2 \\
\hline 9 & 16022 & 8106252 & HEXB & hexosaminidase B (beta polypeptide) \\
\hline 9 & 16160 & 8108378 & CTNNA1 & catenin (cadherin-associated protein), alpha 1, 10 \\
\hline 9 & 16492 & 8112841 & HOMER1 & homer homolog 1 (Drosophila) \\
\hline 9 & 17145 & 8121257 & PRDM1 & PR domain containing 1 , with ZNF domain \\
\hline 9 & 17231 & 8122336 & C6orf115 & chromosome 6 open reading frame 115 \\
\hline 9 & 17345 & 8123961 & TBC1D7 & TBC1 domain family, member 7 \\
\hline 9 & 17683 & 8128123 & RRAGD & Ras-related GTP binding D \\
\hline 9 & 17784 & 8129804 & MAP3K5 & mitogen-activated protein kinase kinase kinase 5 \\
\hline 9 & 17787 & 8129861 & IFNGR1 & interferon gamma receptor 1 \\
\hline 9 & 19810 & 8155930 & GCNT1 & glucosaminyl ( $\mathrm{N}$-acetyl) transferase 1 , core 2 \\
\hline 9 & 20881 & 8169541 & DOCK11 & dedicator of cytokinesis 11 \\
\hline 10 & 1973 & 7922474 & KIAA0040 & KIAA0040 \\
\hline 10 & 42 & 7897210 & DFFB & DNA fragmentation factor, $40 \mathrm{kDa}$, beta polypeptide \\
\hline 10 & 354 & 7901102 & MMACHC & methylmalonic aciduria (cobalamin deficiency) cblC \\
\hline 10 & 661 & 7905299 & PRUNE & prune homolog (Drosophila) \\
\hline 10 & 1312 & 7913787 & C1orf201 & chromosome 1 open reading frame 201 \\
\hline 10 & 1646 & 7918457 & KCNA3 & potassium voltage-gated channel, shaker-related su \\
\hline 10 & 2529 & 7930139 & TRIM8 & tripartite motif-containing 8 \\
\hline 10 & 3396 & 7941104 & ARL2 & ADP-ribosylation factor-like 2 \\
\hline 10 & 3505 & 7942562 & NEU3 & sialidase 3 (membrane sialidase) \\
\hline 10 & 3762 & 7945875 & FAM86C & family with sequence similarity 86 , member $C$ \\
\hline 10 & 4138 & 7949948 & C11orf24 & chromosome 11 open reading frame 24 \\
\hline 10 & 4931 & 7960320 & DCP1B & DCP1 decapping enzyme homolog B (S. cerevisiae) \\
\hline 10 & 6055 & 7975926 & KIAA1737 & KIAA1737 \\
\hline 10 & 6343 & 7979505 & SIX1 & SIX homeobox 1 \\
\hline 10 & 6910 & 7986463 & LRRK1 & leucine-rich repeat kinase 1 \\
\hline 10 & 7308 & 7992594 & CCNF & cyclin F \\
\hline 10 & 7736 & 7998336 & NARFL & nuclear prelamin A recognition factor-like \\
\hline 10 & 8540 & 8008297 & XYLT2 & xylosyltransferase II \\
\hline 10 & 8543 & 8008339 & RSAD1 & radical S-adenosyl methionine domain containing 1 \\
\hline 10 & 8764 & 8011396 & SHPK & sedoheptulokinase \\
\hline 10 & 9234 & 8017186 & HEATR6 & HEAT repeat containing 6 \\
\hline 10 & 9986 & 8026712 & ANKLE1 & ankyrin repeat and LEM domain containing 1 \\
\hline 10 & 16165 & 8108447 & CXXC5 & CXXC finger 5 \\
\hline 10 & 17196 & 8121850 & HEY2 & hairy/enhancer-of-split related with YRPW motif 2 \\
\hline 10 & 18410 & 8138088 & C7orf70 & chromosome 7 open reading frame 70 \\
\hline 10 & 20320 & 8162438 & $\mathrm{BICD} 2$ & bicaudal D homolog 2 (Drosophila) \\
\hline 10 & 20457 & 8164252 & $\mathrm{SH} 2 \mathrm{D} 3 \mathrm{C}$ & $\mathrm{SH} 2$ domain containing $3 \mathrm{C}$ \\
\hline
\end{tabular}




\begin{tabular}{|c|c|c|c|c|}
\hline 10 & 20461 & 8164314 & ST6GALNAC4 & ST6 (alpha-N-acetyl-neuraminyl-2,3-beta-galactosyl \\
\hline 10 & 20489 & 8164644 & FAM78A & family with sequence similarity 78 , member $A$ \\
\hline 10 & 21991 & 8180413 & ST6GALNAC4 & ST6 (alpha-N-acetyl-neuraminyl-2,3-beta-galactosyl \\
\hline 11 & 19176 & 8148059 & DEPDC6 & DEP domain containing 6 \\
\hline 11 & 405 & 7901788 & NFIA & nuclear factor $\mathrm{I} / \mathrm{A}$ \\
\hline 11 & 771 & 7906386 & PYHIN1 & pyrin and HIN domain family, member 1 \\
\hline 11 & 943 & 7908924 & PRELP & proline/arginine-rich end leucine-rich repeat prot \\
\hline 11 & 1307 & 7913705 & CNR2 & cannabinoid receptor 2 (macrophage) \\
\hline 11 & 2311 & 7927215 & ALOX5 & arachidonate 5-lipoxygenase \\
\hline 11 & 3096 & 7938035 & TRIM22 & tripartite motif-containing 22 \\
\hline 11 & 4061 & 7949021 & RCOR2 & REST corepressor 2 \\
\hline 11 & 4168 & 7950307 & UCP2 & uncoupling protein 2 (mitochondrial, proton carrie \\
\hline 11 & 4208 & 7950810 & SYTL2 & synaptotagmin-like 2 \\
\hline 11 & 4809 & 7958466 & ACACB & acetyl-CoA carboxylase beta \\
\hline 11 & 5003 & 7961175 & KLRC3 & killer cell lectin-like receptor subfamily $C$, memb \\
\hline 11 & 6172 & 7977319 & PLD4 & phospholipase D family, member 4 \\
\hline 11 & 6263 & 7978376 & STXBP6 & syntaxin binding protein 6 (amisyn) \\
\hline 11 & 6460 & 7981142 & CLMN & calmin (calponin-like, transmembrane) \\
\hline 11 & 7111 & 7990033 & TLE3 & transducin-like enhancer of split 3 (E(sp1) homolo \\
\hline 11 & 7847 & 7999674 & MYH11 & myosin, heavy chain 11 , smooth muscle \\
\hline 11 & 9261 & 8017599 & PECAM1 & platelet/endothelial cell adhesion molecule \\
\hline 11 & 10571 & 8033233 & TUBB4 & tubulin, beta 4 \\
\hline 11 & 10909 & 8037205 & CEACAM1 & carcinoembryonic antigen-related cell adhesion mol \\
\hline 11 & 11579 & 8045182 & PTPN18 & protein tyrosine phosphatase, non-receptor type 18 \\
\hline 11 & 12387 & 8057418 & ZNF385B & zinc finger protein 385B \\
\hline 11 & 13106 & 8067185 & BMP7 & bone morphogenetic protein 7 \\
\hline 11 & 13692 & 8074237 & CECR1 & cat eye syndrome chromosome region, candidate 1 \\
\hline 11 & 14484 & 8084742 & LPP & LIM domain containing preferred translocation part \\
\hline 11 & 16031 & 8106411 & S100Z & S100 calcium binding protein Z \\
\hline 11 & 16542 & 8113504 & C5orf13 & chromosome 5 open reading frame 13 \\
\hline 11 & 17432 & 8124806 & NRM & nurim (nuclear envelope membrane protein) \\
\hline 11 & 19841 & 8156321 & SYK & spleen tyrosine kinase \\
\hline 11 & 21058 & 8171493 & CTPS2 & CTP synthase II \\
\hline 12 & 9656 & 8022817 & KLHL14 & kelch-like 14 (Drosophila) \\
\hline 12 & 1867 & 7921319 & FCRL1 & Fc receptor-like 1 \\
\hline 12 & 2779 & 7933760 & CCDC6 & coiled-coil domain containing 6 \\
\hline 12 & 2780 & 7933772 & ANK3 & ankyrin 3, node of Ranvier (ankyrin G) \\
\hline 12 & 4014 & 7948565 & CYBASC3 & cytochrome b, ascorbate dependent 3 \\
\hline 12 & 4491 & 7954104 & ATF7IP & activating transcription factor 7 interacting prot \\
\hline 12 & 5077 & 7962151 & DENND5B & DENN/MADD domain containing 5B \\
\hline 12 & 5323 & 7965541 & FGD6 & FYVE, RhoGEF and PH domain containing 6 \\
\hline 12 & 5596 & 7969677 & MBNL2 & muscleblind-like 2 (Drosophila) \\
\hline 12 & 5972 & 7974653 & KIAA0586 & KIAA0586 \\
\hline 12 & 6431 & 7980744 & RPS6KA5 & ribosomal protein S6 kinase, $90 \mathrm{kDa}$, polypeptide 5 \\
\hline 12 & 6900 & 7986383 & IGF1R & insulin-like growth factor 1 receptor \\
\hline 12 & 6995 & 7987892 & ZFP106 & zinc finger protein 106 homolog (mouse) \\
\hline 12 & 8469 & 8007471 & NBR1 & neighbor of BRCA1 gene 1 \\
\hline 12 & 9186 & 8016546 & ZNF652 & zinc finger protein 652 \\
\hline 12 & 11738 & 8047565 & FAM117B & family with sequence similarity 117 , member $B$ \\
\hline 12 & 14752 & 8088550 & PRICKLE2 & prickle homolog 2 (Drosophila) \\
\hline
\end{tabular}




\begin{tabular}{|c|c|c|c|c|}
\hline 12 & 14794 & 8089261 & CBLB & Cas-Br-M (murine) ecotropic retroviral transformin \\
\hline 12 & 15401 & 8097657 & SMAD1 & SMAD family member 1 \\
\hline 12 & 16038 & 8106516 & JMY & junction mediating and regulatory protein, p53 cof \\
\hline 12 & 16437 & 8112107 & PPAP2A & phosphatidic acid phosphatase type $2 \mathrm{~A}$ \\
\hline 12 & 17003 & 8119161 & PIM1 & pim-1 oncogene \\
\hline 12 & 17209 & 8122071 & ENPP3 & ectonucleotide pyrophosphatase/phosphodiesterase 3 \\
\hline 12 & 18799 & 8142930 & TSGA14 & testis specific, 14 \\
\hline 12 & 20331 & 8162533 & PTCH1 & patched 1 \\
\hline 12 & 20348 & 8162719 & HEMGN & hemogen \\
\hline 12 & 20648 & 8166730 & CYBB & cytochrome b-245, beta polypeptide \\
\hline 12 & 21096 & 8172088 & BCOR & BCL6 corepressor \\
\hline 12 & 21127 & 8172471 & PIM2 & pim-2 oncogene \\
\hline 12 & 21228 & 8173869 & POF1B & premature ovarian failure, $1 \mathrm{~B}$ \\
\hline 13 & 20406 & 8163629 & TNFSF8 & tumor necrosis factor (ligand) superfamily, member \\
\hline 13 & 265 & 7899870 & ZNF362 & zinc finger protein 362 \\
\hline 13 & 623 & 7904907 & BCL9 & B-cell CLL/lymphoma 9 \\
\hline 13 & 2364 & 7927876 & TET1 & tet oncogene 1 \\
\hline 13 & 2650 & 7931863 & ASB13 & ankyrin repeat and SOCS box-containing 13 \\
\hline 13 & 2655 & 7931930 & PRKCQ & protein kinase $\mathrm{C}$, theta \\
\hline 13 & 2904 & 7935660 & DNMBP & dynamin binding protein \\
\hline 13 & 3922 & 7947624 & PHF21A & PHD finger protein $21 \mathrm{~A}$ \\
\hline 13 & 4073 & 7949206 & MEN1 & multiple endocrine neoplasia I \\
\hline 13 & 4163 & 7950248 & FCHSD2 & $\mathrm{FCH}$ and double $\mathrm{SH} 3$ domains 2 \\
\hline 13 & 4704 & 7956878 & IRAK3 & interleukin-1 receptor-associated kinase 3 \\
\hline 13 & 4720 & 7957126 & KCNMB4 & potassium large conductance calcium-activated chan \\
\hline 13 & 4807 & 7958439 & USP30 & ubiquitin specific peptidase 30 \\
\hline 13 & 4838 & 7958960 & TPCN1 & two pore segment channel 1 \\
\hline 13 & 4843 & 7959025 & RNFT2 & ring finger protein, transmembrane 2 \\
\hline 13 & 5277 & 7964852 & BEST3 & bestrophin 3 \\
\hline 13 & 5363 & 7966183 & ALKBH2 & alkB, alkylation repair homolog 2 (E. coli) \\
\hline 13 & 5688 & 7971015 & SMAD9 & SMAD family member 9 \\
\hline 13 & 7059 & 7989159 & ZNF280D & zinc finger protein $280 \mathrm{D}$ \\
\hline 13 & 10640 & 8034130 & KANK2 & KN motif and ankyrin repeat domains 2 \\
\hline 13 & 11313 & 8041617 & MTA3 & metastasis associated 1 family, member 3 \\
\hline 13 & 12515 & 8059650 & SP110 & SP110 nuclear body protein \\
\hline 13 & 13235 & 8068671 & BACE2 & beta-site APP-cleaving enzyme 2 \\
\hline 13 & 13820 & 8075673 & RBM9 & RNA binding motif protein 9 \\
\hline 13 & 14721 & 8088142 & $\mathrm{CHDH}$ & choline dehydrogenase \\
\hline 13 & 16500 & 8112940 & SSBP2 & single-stranded DNA binding protein 2 \\
\hline 13 & 16854 & 8117476 & BTN3A3 & butyrophilin, subfamily 3 , member $A 3$ \\
\hline 13 & 17590 & 8126770 & CYP39A1 & cytochrome P450, family 39 , subfamily A, polypepti \\
\hline 13 & 19533 & 8152133 & RRM2B & ribonucleotide reductase M2 B (TP53 inducible) \\
\hline 13 & 21179 & 8173208 & SPIN4 & spindlin family, member 4 \\
\hline 16 & 16265 & 8109649 & NA & NA \\
\hline 16 & 81 & 7897774 & CLCN6 & chloride channel 6 \\
\hline 16 & 1082 & 7910950 & KMO & kynurenine 3-monooxygenase (kynurenine 3-hydroxyla \\
\hline 16 & 2785 & 7933877 & JMJD1C & jumonji domain containing $1 \mathrm{C}$ \\
\hline 16 & 4469 & 7953878 & CLEC2D & C-type lectin domain family 2 , member $D$ \\
\hline 16 & 5000 & 7961151 & KLRK1 & killer cell lectin-like receptor subfamily $\mathrm{K}$, memb \\
\hline 16 & 5100 & 7962516 & SLC38A1 & solute carrier family 38 , member 1 \\
\hline
\end{tabular}




\begin{tabular}{|l|l|l|l|l|}
\hline $\mathbf{1 6}$ & 6023 & 7975545 & PSEN1 & presenilin 1 \\
\hline $\mathbf{1 6}$ & 6843 & 7985482 & WHAMM & WAS protein homolog associated with actin, golgi m \\
\hline $\mathbf{1 6}$ & $\mathbf{7 5 5 2}$ & 7995895 & HERPUD1 & homocysteine-inducible, endoplasmic reticulum stre \\
\hline $\mathbf{1 6}$ & 8319 & 8005765 & WSB1 & WD repeat and SOCS box-containing 1 \\
\hline $\mathbf{1 6}$ & 9576 & 8021653 & SERPINB8 & serpin peptidase inhibitor, clade B (ovalbumin), m \\
\hline $\mathbf{1 6}$ & 11228 & 8040340 & LPIN1 & lipin 1 \\
\hline $\mathbf{1 6}$ & 11234 & 8040440 & GEN1 & Gen homolog 1, endonuclease (Drosophila) \\
\hline $\mathbf{1 6}$ & 11300 & 8041447 & CRIM1 & cysteine rich transmembrane BMP regulator 1 (chord \\
\hline $\mathbf{1 6}$ & 12920 & 8064868 & GPCPD1 & glycerophosphocholine phosphodiesterase GDE1 homol \\
\hline $\mathbf{1 6}$ & 13182 & 8068105 & BACH1 & BTB and CNC homology 1, basic leucine zipper trans \\
\hline $\mathbf{1 6}$ & 14333 & 8082607 & ATP2C1 & ATPase, Ca++ transporting, type 2C, member 1 \\
\hline $\mathbf{1 6}$ & 15718 & 8101992 & SLC39A8 & solute carrier family 39 (zinc transporter), membe \\
\hline $\mathbf{1 6}$ & 15744 & 8102389 & C4orf21 & chromosome 4 open reading frame 21 \\
\hline $\mathbf{1 6}$ & 15974 & 8105506 & ZSWIM6 & zinc finger, SWIM-type containing 6 \\
\hline $\mathbf{1 6}$ & 16569 & 8113914 & FNIP1 & folliculin interacting protein 1 \\
\hline $\mathbf{1 6}$ & 18369 & 8137526 & INSIG1 & insulin induced gene 1 \\
\hline $\mathbf{1 6}$ & 19031 & 8145954 & TACC1 & transforming, acidic coiled-coil containing protei \\
\hline $\mathbf{1 6}$ & 19076 & 8146550 & SDCBP & syndecan binding protein (syntenin) \\
\hline $\mathbf{1 6}$ & 19905 & 8157216 & UGCG & UDP-glucose ceramide glucosyltransferase \\
\hline $\mathbf{1 6}$ & 19926 & 8157524 & TLR4 & toll-like receptor 4 \\
\hline $\mathbf{1 6}$ & 20355 & 8162833 & ERP44 & endoplasmic reticulum protein 44 \\
\hline $\mathbf{1 6}$ & 20495 & 8164701 & SETX & senataxin \\
\hline $\mathbf{1 6}$ & 21856 & 8180268 & CYP51A1 & cytochrome P450, family 51, subfamily A, polypepti \\
\hline & & & &
\end{tabular}

Table A-4 Testing for stationarity reveals 5 distinct and disjoint groups of genes, changing their expression after SCD40L stimulation.

\begin{tabular}{|r|r|c|c|l|}
\hline & Gene ID & Probe ID & Gene Symbol & Gene Name \\
\hline $\mathbf{2}$ & 16514 & 8113073 & ARRDC3 & arrestin domain containing 3 \\
\hline $\mathbf{2}$ & 903 & 7908376 & RGS18 & regulator of G-protein signaling 18 \\
\hline $\mathbf{2}$ & 905 & 7908388 & RGS1 & regulator of G-protein signaling 1 \\
\hline $\mathbf{2}$ & 908 & 7908409 & RGS2 & regulator of G-protein signaling 2, 24kDa \\
\hline $\mathbf{2}$ & 1897 & 7921625 & SLAMF6 & SLAM family member 6 \\
\hline $\mathbf{2}$ & 2985 & 7936884 & FAM53B & family with sequence similarity 53, member B \\
\hline $\mathbf{2}$ & 4441 & 7953532 & ENO2 & enolase 2 (gamma, neuronal) \\
\hline $\mathbf{2}$ & 4545 & 7954926 & PDZRN4 & PDZ domain containing ring finger 4 \\
\hline $\mathbf{2}$ & 4992 & 7961075 & CD69 & CD69 molecule \\
\hline $\mathbf{2}$ & 5363 & 7966183 & ALKBH2 & alkB, alkylation repair homolog 2 (E. coli) \\
\hline $\mathbf{2}$ & 8674 & 8010139 & SEC14L1 & SEC14-like 1 (S. cerevisiae) \\
\hline $\mathbf{2}$ & 9011 & 8014706 & PCGF2 & polycomb group ring finger 2 \\
\hline $\mathbf{2}$ & 12671 & 8061564 & ID1 & inhibitor of DNA binding 1, dominant negative heli \\
\hline $\mathbf{2}$ & 13494 & 8071691 & BCR & breakpoint cluster region \\
\hline $\mathbf{2}$ & 14598 & 8086330 & CSRNP1 & cysteine-serine-rich nuclear protein 1 \\
\hline $\mathbf{2}$ & 15035 & 8092691 & BCL6 & B-cell CLL/lymphoma 6 \\
\hline $\mathbf{2}$ & 15296 & 8096070 & BMP3 & bone morphogenetic protein 3 \\
\hline $\mathbf{2}$ & 15668 & 8101322 & MOP-1 & MOP-1 \\
\hline $\mathbf{2}$ & 15726 & 8102135 & CXXC4 & CXXC finger 4 \\
\hline & & & & \\
\hline
\end{tabular}




\begin{tabular}{|c|c|c|c|c|}
\hline 2 & 15806 & 8103226 & TMEM154 & transmembrane protein 154 \\
\hline 2 & 16475 & 8112615 & ENC1 & ectodermal-neural cortex 1 (with BTB-like domain) \\
\hline 2 & 16684 & 8115490 & ADAM19 & ADAM metallopeptidase domain 19 \\
\hline 2 & 16818 & 8117120 & ID4 & inhibitor of DNA binding 4, dominant negative heli \\
\hline 2 & 17371 & 8124380 & HIST1H1A & histone cluster $1, \mathrm{H} 1 \mathrm{a}$ \\
\hline 2 & 17776 & 8129666 & SLC2A12 & solute carrier family 2 (facilitated glucose trans \\
\hline 2 & 19303 & 8149324 & FAM167A & family with sequence similarity 167 , member $A$ \\
\hline 2 & 19666 & 8154100 & VLDLR & very low density lipoprotein receptor \\
\hline 2 & 20566 & 8165711 & PLCXD1 & phosphatidylinositol-specific phospholipase $\mathrm{C}, \mathrm{X} \mathrm{d}$ \\
\hline 2 & 21237 & 8173999 & XKRX & XK, Kell blood group complex subunit-related, X-li \\
\hline 2 & 21450 & 8176286 & PLCXD1 & phosphatidylinositol-specific phospholipase $\mathrm{C}, \mathrm{X} \mathrm{d}$ \\
\hline 4 & 9890 & 8025601 & ICAM1 & "intercellular adhesion molecule 1 \\
\hline 4 & 287 & 7900146 & ZC3H12A & zinc finger $\mathrm{CCCH}$-type containing $12 \mathrm{~A}$ \\
\hline 4 & 606 & 7904702 & NOTCH2NL & notch $2 \mathrm{~N}$-terminal like \\
\hline 4 & 1348 & 7914212 & SNORA61 & small nucleolar RNA, H/ACA box 61 \\
\hline 4 & 1601 & 7917754 & BCAR3 & breast cancer anti-estrogen resistance 3 \\
\hline 4 & 1697 & 7919193 & NUDT4P1 & nudix (nucleoside diphosphate linked moiety X)-typ \\
\hline 4 & 2287 & 7926900 & MAP3K8 & mitogen-activated protein kinase kinase kinase 8 \\
\hline 4 & 2450 & 7929032 & FAS & Fas (TNF receptor superfamily, member 6) \\
\hline 4 & 4745 & 7957478 & TMTC3 & transmembrane and tetratricopeptide repeat contain \\
\hline 4 & 5448 & 7967456 & RILPL2 & Rab interacting lysosomal protein-like 2 \\
\hline 4 & 5664 & 7970696 & USP12 & ubiquitin specific peptidase 12 \\
\hline 4 & 7434 & 7994292 & IL21R & interleukin 21 receptor \\
\hline 4 & 7833 & 7999496 & ZC3H7A & zinc finger $\mathrm{CCCH}$-type containing 7A \\
\hline 4 & 9034 & 8015031 & CCR7 & chemokine ( $\mathrm{C}-\mathrm{C}$ motif) receptor 7 \\
\hline 4 & 9790 & 8024485 & GADD45B & growth arrest and DNA-damage-inducible, beta \\
\hline 4 & 11145 & 8039545 & NLRP11 & NLR family, pyrin domain containing 11 \\
\hline 4 & 13173 & 8068022 & MIR155HG & MIR155 host gene (non-protein coding) \\
\hline 4 & 13294 & 8069541 & SAMSN1 & SAM domain, SH3 domain and nuclear localization si \\
\hline 4 & 15333 & 8096635 & NFKB1 & nuclear factor of kappa light polypeptide gene enh \\
\hline 4 & 15907 & 8104580 & FAM105B & family with sequence similarity 105 , member $B$ \\
\hline 4 & 15908 & 8104590 & LOC100130744 & hypothetical LOC100130744 \\
\hline 4 & 15939 & 8105067 & PTGER4 & prostaglandin E receptor 4 (subtype EP4) \\
\hline 4 & 16365 & 8110886 & MED10 & mediator complex subunit 10 \\
\hline 4 & 16741 & 8116227 & CLK4 & CDC-like kinase 4 \\
\hline 4 & 17221 & 8122202 & MYB & v-myb myeloblastosis viral oncogene homolog (avian \\
\hline 4 & 17353 & 8124059 & NUP153 & nucleoporin $153 \mathrm{kDa}$ \\
\hline 4 & 17583 & 8126666 & NFKBIE & nuclear factor of kappa light polypeptide gene enh \\
\hline 4 & 17901 & 8131292 & RBAK & RB-associated KRAB zinc finger \\
\hline 4 & 18882 & 8143957 & RHEB & Ras homolog enriched in brain \\
\hline 4 & 21908 & 8180323 & USP12 & ubiquitin specific peptidase 12 \\
\hline$\overline{25}$ & 16808 & 8116983 & CD83 & CD83 molecule \\
\hline 5 & 191 & 7899018 & TMEM57 & transmembrane protein 57 \\
\hline 5 & 571 & 7904086 & LRIG2 & leucine-rich repeats and immunoglobulin-like domai \\
\hline 5 & 3578 & 7943424 & $\mathrm{BIRC2}$ & baculoviral IAP repeat-containing 2 \\
\hline
\end{tabular}




\begin{tabular}{|c|c|c|c|c|}
\hline 5 & 4515 & 7954436 & LRMP & lymphoid-restricted membrane protein \\
\hline 5 & 6751 & 7984364 & SMAD3 & SMAD family member 3 \\
\hline 5 & 6825 & 7985259 & ZFAND6 & zinc finger, AN1-type domain 6 \\
\hline 5 & 7846 & 7999642 & KIAA0430 & KIAA0430 \\
\hline 5 & 9656 & 8022817 & KLHL14 & kelch-like 14 (Drosophila) \\
\hline 5 & 10766 & 8035703 & LPAR2 & lysophosphatidic acid receptor 2 \\
\hline 5 & 10812 & 8036207 & NFKBID & nuclear factor of kappa light polypeptide gene enh \\
\hline 5 & 11925 & 8050190 & ADAM17 & ADAM metallopeptidase domain 17 \\
\hline 5 & 12050 & 8052269 & CCDC88A & coiled-coil domain containing $88 \mathrm{~A}$ \\
\hline 5 & 12074 & 8052689 & SPRED2 & sprouty-related, EVH1 domain containing 2 \\
\hline 5 & 12786 & 8063156 & CD40 & CD40 molecule, TNF receptor superfamily member 5 \\
\hline 5 & 12796 & 8063351 & SLC9A8 & solute carrier family 9 (sodium/hydrogen exchanger \\
\hline 5 & 14291 & 8082035 & CD86 & CD86 molecule \\
\hline 5 & 14449 & 8084219 & KLHL24 & kelch-like 24 (Drosophila) \\
\hline 5 & 14752 & 8088550 & PRICKLE2 & prickle homolog 2 (Drosophila) \\
\hline 5 & 14763 & 8088776 & FOXP1 & forkhead box P1 \\
\hline 5 & 16637 & 8114814 & NR3C1 & nuclear receptor subfamily 3 , group $\mathrm{C}$, member 1 (g \\
\hline 5 & 17209 & 8122071 & ENPP3 & ectonucleotide pyrophosphatase/phosphodiesterase 3 \\
\hline 5 & 17347 & 8123981 & C6orf114 & chromosome 6 open reading frame 114 \\
\hline 5 & 18983 & 8145136 & PPP3CC & protein phosphatase 3 , catalytic subunit, gamma is \\
\hline 5 & 19342 & 8149720 & EGR3 & early growth response 3 \\
\hline 5 & 19514 & 8151890 & TP53INP1 & tumor protein p53 inducible nuclear protein 1 \\
\hline 5 & 19876 & 8156848 & NR4A3 & nuclear receptor subfamily 4, group A, member 3 \\
\hline 5 & 20354 & 8162827 & ALG2 & asparagine-linked glycosylation 2, alpha-1,3-manno \\
\hline 5 & 21127 & 8172471 & PIM2 & pim-2 oncogene \\
\hline 5 & 21458 & 8176384 & ZFY & zinc finger protein, $Y$-linked \\
\hline 7 & 14277 & 8081838 & ARHGAP31 & Rho GTPase activating protein 31 \\
\hline 7 & 65 & 7897482 & PIK3CD & phosphoinositide-3-kinase, catalytic, delta polype \\
\hline 7 & 533 & 7903592 & KIAA1324 & KIAA1324 \\
\hline 7 & 785 & 7906564 & PEA15 & phosphoprotein enriched in astrocytes 15 \\
\hline 7 & 1956 & 7922343 & TNFSF4 & tumor necrosis factor (ligand) superfamily, member \\
\hline 7 & 2819 & 7934367 & ANXA7 & annexin A7 \\
\hline 7 & 4468 & 7953873 & OVOS & ovostatin \\
\hline 7 & 5122 & 7962884 & RND1 & Rho family GTPase 1 \\
\hline 7 & 5147 & 7963235 & CSRNP2 & cysteine-serine-rich nuclear protein 2 \\
\hline 7 & 5377 & 7966356 & HVCN1 & hydrogen voltage-gated channel 1 \\
\hline 7 & 6099 & 7976556 & C14orf132 & chromosome 14 open reading frame 132 \\
\hline 7 & 8934 & 8013633 & UNC119 & unc-119 homolog (C. elegans) \\
\hline 7 & 9798 & 8024572 & GNA15 & guanine nucleotide binding protein (G protein), al \\
\hline 7 & 10628 & 8033996 & TYK2 & tyrosine kinase 2 \\
\hline 7 & 11728 & 8047381 & CFLAR & CASP8 and FADD-like apoptosis regulator \\
\hline 7 & 12201 & 8054192 & MITD1 & MIT, microtubule interacting and transport, domain \\
\hline 7 & 12503 & 8059413 & DOCK10 & dedicator of cytokinesis 10 \\
\hline 7 & 12914 & 8064790 & RASSF2 & Ras association (RaIGDS/AF-6) domain family member \\
\hline 7 & 12942 & 8065230 & RBBP9 & retinoblastoma binding protein 9 \\
\hline
\end{tabular}




\begin{tabular}{|c|c|c|c|c|}
\hline 7 & 13575 & 8072757 & CSF2RB & colony stimulating factor 2 receptor, beta, low-af \\
\hline 7 & 14725 & 8088180 & WNT5A & wingless-type MMTV integration site family, member \\
\hline 7 & 15291 & 8096004 & BMP2K & BMP2 inducible kinase \\
\hline 7 & 16002 & 8105949 & SERF1A & small EDRK-rich factor $1 \mathrm{~A}$ (telomeric) \\
\hline 7 & 16007 & 8105997 & SERF1A & small EDRK-rich factor 1A (telomeric) \\
\hline 7 & 18070 & 8133459 & CLIP2 & CAP-GLY domain containing linker protein 2 \\
\hline 7 & 19590 & 8153043 & ZFAT & zinc finger and AT hook domain containing \\
\hline 7 & 20513 & 8164967 & VAV2 & vav 2 guanine nucleotide exchange factor \\
\hline 7 & 21565 & 8177658 & SERF1A & small EDRK-rich factor $1 \mathrm{~A}$ (telomeric) \\
\hline 7 & 21689 & 8178826 & NA & NA \\
\hline 7 & 21810 & 8180022 & NA & NA \\
\hline 8 & 15667 & 8101304 & RASGEF1B & RasGEF domain family, member 1B \\
\hline 8 & 430 & 7902227 & GADD45A & growth arrest and DNA-damage-inducible, alpha \\
\hline 8 & 974 & 7909332 & CD55 & CD55 molecule, decay accelerating factor for compl \\
\hline 8 & 1992 & 7922717 & RGS16 & regulator of G-protein signaling 16 \\
\hline 8 & 3740 & 7945652 & KRTAP5-3 & keratin associated protein 5-3 \\
\hline 8 & 5000 & 7961151 & KLRK1 & killer cell lectin-like receptor subfamily $\mathrm{K}$, memb \\
\hline 8 & 5101 & 7962537 & SLC38A2 & solute carrier family 38 , member 2 \\
\hline 8 & 5313 & 7965423 & BTG1 & B-cell translocation gene 1 , anti-proliferative \\
\hline 8 & 6135 & 7976844 & NA & NA \\
\hline 8 & 6366 & 7979813 & ZFP36L1 & zinc finger protein $36, \mathrm{C} 3 \mathrm{H}$ type-like 1 \\
\hline 8 & 9540 & 8021183 & SCARNA17 & small Cajal body-specific RNA 17 \\
\hline 8 & 9935 & 8026163 & IER2 & immediate early response 2 \\
\hline 8 & 11377 & 8042503 & MXD1 & MAX dimerization protein 1 \\
\hline 8 & 11866 & 8049530 & LRRFIP1 & leucine rich repeat (in FLII) interacting protein \\
\hline 8 & 12150 & 8053576 & RNF103 & ring finger protein 103 \\
\hline 8 & 12159 & 8053668 & EIF2AK3 & eukaryotic translation initiation factor 2 -alpha $\mathrm{k}$ \\
\hline 8 & 12343 & 8056359 & COBLL1 & COBL-like 1 \\
\hline 8 & 15560 & 8099834 & TLR1 & toll-like receptor 1 \\
\hline 8 & 15741 & 8102362 & TIFA & TRAF-interacting protein with forkhead-associated \\
\hline 8 & 16292 & 8110032 & C5orf41 & chromosome 5 open reading frame 41 \\
\hline 8 & 17751 & 8129317 & SERINC1 & serine incorporator 1 \\
\hline 8 & 17832 & 8130539 & TAGAP & T-cell activation RhoGTPase activating protein \\
\hline 8 & 18203 & 8135392 & HBP1 & HMG-box transcription factor 1 \\
\hline 8 & 18750 & 8142307 & PNPLA8 & patatin-like phospholipase domain containing 8 \\
\hline 8 & 19111 & 8147040 & ZBTB10 & zinc finger and BTB domain containing 10 \\
\hline 8 & 19147 & 8147573 & OSR2 & odd-skipped related 2 (Drosophila) \\
\hline 8 & 19380 & 8150186 & RNF122 & ring finger protein 122 \\
\hline 8 & 20215 & 8161224 & ZBTB5 & zinc finger and BTB domain containing 5 \\
\hline 8 & 20368 & 8162940 & ABCA1 & ATP-binding cassette, sub-family A ( $A B C 1)$, member \\
\hline 8 & 21032 & 8171205 & NLGN4X & neuroligin 4, X-linked \\
\hline
\end{tabular}


Table A-5 Testing for stationarity reveals 15 distinct and disjoint groups of genes in BCR-resolved time series analysis. Each group contains 30 genes, which are listed below. Each group of genes displays a unique expression course.

\begin{tabular}{|c|c|c|c|c|}
\hline & Gene ID & Probe ID & Gene Symbol & Gene Name \\
\hline 1 & 790 & 7906613 & SLAMF7 & SLAM family member 7 \\
\hline 1 & 587 & 7904361 & FAM46C & family with sequence similarity 46 , member $C$ \\
\hline 1 & 762 & 7906339 & CD1A & CD1a molecule \\
\hline 1 & 763 & 7906348 & CD1C & CD1c molecule \\
\hline 1 & 1056 & 7910427 & GALNT2 & UDP-N-acetyl-alpha-D-galactosamine:polypeptide $\mathrm{N}$-a \\
\hline 1 & 1169 & 7911754 & TNFRSF14 & tumor necrosis factor receptor superfamily, member \\
\hline 1 & 3735 & 7945620 & TOLLIP & toll interacting protein \\
\hline 1 & 3995 & 7948332 & LPXN & leupaxin \\
\hline 1 & 5286 & 7965040 & PHLDA1 & pleckstrin homology-like domain, family A, member \\
\hline 1 & 6630 & 7982564 & SPRED1 & sprouty-related, EVH1 domain containing 1 \\
\hline 1 & 6730 & 7984112 & RAB8B & RAB8B, member RAS oncogene family \\
\hline 1 & 7381 & 7993433 & PDXDC1 & pyridoxal-dependent decarboxylase domain containin \\
\hline 1 & 8319 & 8005765 & WSB1 & WD repeat and SOCS box-containing 1 \\
\hline 1 & 8623 & 8009476 & MAP2K6 & mitogen-activated protein kinase kinase 6 \\
\hline 1 & 9716 & 8023646 & $\mathrm{BCL} 2$ & B-cell CLL/lymphoma 2 \\
\hline 1 & 12007 & 8051547 & PRKD3 & protein kinase D3 \\
\hline 1 & 12017 & 8051707 & MAP4K3 & mitogen-activated protein kinase kinase kinase kin \\
\hline 1 & 12534 & 8059854 & ARL4C & ADP-ribosylation factor-like $4 \mathrm{C}$ \\
\hline 1 & 14295 & 8082075 & DTX3L & deltex 3-like (Drosophila) \\
\hline 1 & 14296 & 8082086 & PARP15 & poly (ADP-ribose) polymerase family, member 15 \\
\hline 1 & 14768 & 8088848 & PDZRN3 & PDZ domain containing ring finger 3 \\
\hline 1 & 15587 & 8100231 & TEC & tec protein tyrosine kinase \\
\hline 1 & 17286 & 8123181 & IGF2R & insulin-like growth factor 2 receptor \\
\hline 1 & 17428 & 8124742 & GNL1 & guanine nucleotide binding protein-like 1 \\
\hline 1 & 18005 & 8132725 & UPP1 & uridine phosphorylase 1 \\
\hline 1 & 18360 & 8137404 & CHPF2 & chondroitin polymerizing factor 2 \\
\hline 1 & 18952 & 8144658 & NEIL2 & nei endonuclease VIII-like 2 (E. coli) \\
\hline 1 & 19304 & 8149330 & CTSB & cathepsin B \\
\hline 1 & 19510 & 8151816 & GEM & GTP binding protein overexpressed in skeletal musc \\
\hline 1 & 20495 & 8164701 & SETX & senataxin \\
\hline 2 & 9034 & 8015031 & CCR7 & chemokine (C-C motif) receptor 7 \\
\hline 2 & 791 & 7906622 & LY9 & lymphocyte antigen 9 \\
\hline 2 & 2110 & 7924450 & DUSP10 & dual specificity phosphatase 10 \\
\hline 2 & 2548 & 7930413 & DUSP5 & dual specificity phosphatase 5 \\
\hline 2 & 4668 & 7956287 & NAB2 & NGFI-A binding protein 2 (EGR1 binding protein 2) \\
\hline 2 & 5377 & 7966356 & HVCN1 & hydrogen voltage-gated channel 1 \\
\hline 2 & 5409 & 7966929 & RAB35 & RAB35, member RAS oncogene family \\
\hline 2 & 7405 & 7993825 & RUNDC2B & RUN domain containing 2B \\
\hline 2 & 7452 & 7994576 & RUNDC2C & RUN domain containing $2 \mathrm{C}$ \\
\hline 2 & 8886 & 8013071 & FLCN & folliculin \\
\hline 2 & 9379 & 8019308 & MAFG & v-maf musculoaponeurotic fibrosarcoma oncogene hom \\
\hline
\end{tabular}




\begin{tabular}{|c|c|c|c|c|}
\hline 2 & 9440 & 8019796 & MAFG & v-maf musculoaponeurotic fibrosarcoma oncogene hom \\
\hline 2 & 9947 & 8026300 & CD97 & CD97 molecule \\
\hline 2 & 11228 & 8040340 & LPIN1 & lipin 1 \\
\hline 2 & 12770 & 8062964 & SYS1 & SYS1 Golgi-localized integral membrane protein hom \\
\hline 2 & 13394 & 8070720 & ICOSLG & inducible T-cell co-stimulator ligand \\
\hline 2 & 13964 & 8077441 & BHLHE40 & basic helix-loop-helix family, member e40 \\
\hline 2 & 14505 & 8084951 & LRRC33 & leucine rich repeat containing 33 \\
\hline 2 & 15939 & 8105067 & PTGER4 & prostaglandin E receptor 4 (subtype EP4) \\
\hline 2 & 16336 & 8110569 & SQSTM1 & sequestosome 1 \\
\hline 2 & 16569 & 8113914 & FNIP1 & folliculin interacting protein 1 \\
\hline 2 & 16572 & 8114010 & IRF1 & interferon regulatory factor 1 \\
\hline 2 & 16748 & 8116316 & MGAT4B & mannosyl (alpha-1,3-)-glycoprotein beta-1,4-N-acet \\
\hline 2 & 17583 & 8126666 & NFKBIE & nuclear factor of kappa light polypeptide gene enh \\
\hline 2 & 17677 & 8128043 & CNR1 & cannabinoid receptor 1 (brain) \\
\hline 2 & 18369 & 8137526 & INSIG1 & insulin induced gene 1 \\
\hline 2 & 19031 & 8145954 & TACC1 & transforming, acidic coiled-coil containing protei \\
\hline 2 & 19589 & 8153021 & ST3GAL1 & ST3 beta-galactoside alpha-2,3-sialyltransferase 1 \\
\hline 2 & 19677 & 8154233 & CD274 & CD274 molecule \\
\hline 2 & 19905 & 8157216 & UGCG & UDP-glucose ceramide glucosyltransferase \\
\hline 3 & 18760 & 8142452 & TFEC & "transcription factor EC \\
\hline 3 & 716 & 7905789 & IL6R & interleukin 6 receptor \\
\hline 3 & 1601 & 7917754 & BCAR3 & breast cancer anti-estrogen resistance 3 \\
\hline 3 & 1864 & 7921259 & FCRL4 & Fc receptor-like 4 \\
\hline 3 & 4755 & 7957570 & PLXNC1 & plexin C1 \\
\hline 3 & 5544 & 7968976 & LRCH1 & leucine-rich repeats and calponin homology $(\mathrm{CH})$ do \\
\hline 3 & 5549 & 7969060 & FNDC3A & fibronectin type III domain containing $3 \mathrm{~A}$ \\
\hline 3 & 7869 & 8000028 & DCUN1D3 & DCN1, defective in cullin neddylation 1 , domain co \\
\hline 3 & 9930 & 8026106 & CALR & calreticulin \\
\hline 3 & 10652 & 8034304 & ACP5 & acid phosphatase 5 , tartrate resistant \\
\hline 3 & 11686 & 8046726 & SSFA2 & sperm specific antigen 2 \\
\hline 3 & 12500 & 8059361 & WDFY1 & WD repeat and FYVE domain containing 1 \\
\hline 3 & 13383 & 8070584 & TMPRSS3 & transmembrane protease, serine 3 \\
\hline 3 & 14155 & 8080084 & MANF & mesencephalic astrocyte-derived neurotrophic facto \\
\hline 3 & 14471 & 8084634 & DNAJB11 & DnaJ (Hsp40) homolog, subfamily B, member 11 \\
\hline 3 & 14874 & 8090490 & RPN1 & ribophorin I \\
\hline 3 & 15291 & 8096004 & BMP2K & BMP2 inducible kinase \\
\hline 3 & 15358 & 8097017 & UGT8 & UDP glycosyltransferase 8 \\
\hline 3 & 15561 & 8099841 & TLR6 & toll-like receptor 6 \\
\hline 3 & 16022 & 8106252 & HEXB & hexosaminidase B (beta polypeptide) \\
\hline 3 & 16095 & 8107474 & DMXL1 & Dmx-like 1 \\
\hline 3 & 16160 & 8108378 & CTNNA1 & catenin (cadherin-associated protein), alpha 1,10 \\
\hline 3 & 17551 & 8126303 & TREM1 & triggering receptor expressed on myeloid cells 1 \\
\hline 3 & 17683 & 8128123 & RRAGD & Ras-related GTP binding D \\
\hline 3 & 17784 & 8129804 & MAP3K5 & mitogen-activated protein kinase kinase kinase 5 \\
\hline 3 & 17936 & 8131844 & GPNMB & glycoprotein (transmembrane) nmb \\
\hline
\end{tabular}




\begin{tabular}{|c|c|c|c|c|}
\hline 3 & 18217 & 8135576 & TES & testis derived transcript (3 LIM domains) \\
\hline 3 & 19933 & 8157650 & PTGS1 & prostaglandin-endoperoxide synthase 1 (prostagland \\
\hline 3 & 20270 & 8161701 & TMEM2 & transmembrane protein 2 \\
\hline 3 & 20448 & 8164165 & HSPA5 & heat shock $70 \mathrm{kDa}$ protein 5 (glucose-regulated prot \\
\hline 4 & 20135 & 8160332 & MLLT3 & "myeloid/lymphoid or mixed-lineage leukemia (tritho \\
\hline 4 & 771 & 7906386 & PYHIN1 & pyrin and HIN domain family, member 1 \\
\hline 4 & 943 & 7908924 & PRELP & proline/arginine-rich end leucine-rich repeat prot \\
\hline 4 & 2243 & 7926170 & DHTKD1 & dehydrogenase E1 and transketolase domain containi \\
\hline 4 & 2655 & 7931930 & PRKCQ & protein kinase $\mathrm{C}$, theta \\
\hline 4 & 4208 & 7950810 & SYTL2 & synaptotagmin-like 2 \\
\hline 4 & 4478 & 7953981 & ETV6 & ets variant 6 \\
\hline 4 & 4720 & 7957126 & KCNMB4 & potassium large conductance calcium-activated chan \\
\hline 4 & 4843 & 7959025 & RNFT2 & ring finger protein, transmembrane 2 \\
\hline 4 & 6735 & 7984174 & SNX22 & sorting nexin 22 \\
\hline 4 & 6747 & 7984298 & DIS3L & DIS3 mitotic control homolog (S. cerevisiae)-like \\
\hline 4 & 7012 & 7988212 & ELL3 & elongation factor RNA polymerase II-like 3 \\
\hline 4 & 10640 & 8034130 & KANK2 & KN motif and ankyrin repeat domains 2 \\
\hline 4 & 10909 & 8037205 & CEACAM 1 & carcinoembryonic antigen-related cell adhesion mol \\
\hline 4 & 11456 & 8043465 & IGKC & immunoglobulin kappa constant \\
\hline 4 & 12914 & 8064790 & RASSF2 & Ras association (RalGDS/AF-6) domain family member \\
\hline 4 & 13737 & 8074780 & YPEL1 & yippee-like 1 (Drosophila) \\
\hline 4 & 13820 & 8075673 & RBM9 & RNA binding motif protein 9 \\
\hline 4 & 14179 & 8080562 & IL17RB & interleukin 17 receptor $B$ \\
\hline 4 & 14721 & 8088142 & $\mathrm{CHDH}$ & choline dehydrogenase \\
\hline 4 & 16031 & 8106411 & S100Z & S100 calcium binding protein Z \\
\hline 4 & 16829 & 8117243 & LRRC16A & leucine rich repeat containing $16 \mathrm{~A}$ \\
\hline 4 & 18441 & 8138489 & CDCA7L & cell division cycle associated 7-like \\
\hline 4 & 18647 & 8140955 & CDK6 & cyclin-dependent kinase 6 \\
\hline 4 & 18719 & 8141768 & RASA4 & RAS p21 protein activator 4 \\
\hline 4 & 18722 & 8141803 & RASA4 & RAS p21 protein activator 4 \\
\hline 4 & 19845 & 8156393 & SUSD3 & sushi domain containing 3 \\
\hline 4 & 19953 & 8157804 & OLFML2A & olfactomedin-like $2 \mathrm{~A}$ \\
\hline 4 & 20348 & 8162719 & HEMGN & hemogen \\
\hline 4 & 20526 & 8165156 & SDCCAG3 & serologically defined colon cancer antigen 3 \\
\hline 5 & 19876 & 8156848 & NR4A3 & "nuclear receptor subfamily 4, group A, member 3 \\
\hline 5 & 109 & 7898070 & PRDM2 & PR domain containing 2 , with ZNF domain \\
\hline 5 & 300 & 7900365 & MFSD2A & major facilitator superfamily domain containing $2 \mathrm{~A}$ \\
\hline 5 & 1047 & 7910261 & C1orf69 & chromosome 1 open reading frame 69 \\
\hline 5 & 1992 & 7922717 & RGS16 & regulator of G-protein signaling 16 \\
\hline 5 & 2398 & 7928429 & PLAU & plasminogen activator, urokinase \\
\hline 5 & 2643 & 7931810 & KLF6 & Kruppel-like factor 6 \\
\hline 5 & 5789 & 7972548 & GPR18 & G protein-coupled receptor 18 \\
\hline 5 & 6366 & 7979813 & ZFP36L1 & zinc finger protein $36, \mathrm{C} 3 \mathrm{H}$ type-like 1 \\
\hline 5 & 6386 & 7980051 & C14orf43 & chromosome 14 open reading frame 43 \\
\hline 5 & 8665 & 8010050 & FAM100B & family with sequence similarity 100 , member $B$ \\
\hline
\end{tabular}




\begin{tabular}{|c|c|c|c|c|}
\hline 5 & 9935 & 8026163 & IER2 & immediate early response 2 \\
\hline 5 & 10003 & 8026915 & ARRDC2 & arrestin domain containing 2 \\
\hline 5 & 10277 & 8030128 & PPP1R15A & protein phosphatase 1, regulatory (inhibitor) subu \\
\hline 5 & 10744 & 8035445 & JUND & jun $\mathrm{D}$ proto-oncogene \\
\hline 5 & 10812 & 8036207 & NFKBID & nuclear factor of kappa light polypeptide gene enh \\
\hline 5 & 11834 & 8049075 & B3GNT7 & UDP-GIcNAc:betaGal beta-1,3-N-acetylglucosaminyltr \\
\hline 5 & 12107 & 8053022 & EGR4 & early growth response 4 \\
\hline 5 & 12179 & 8053882 & DUSP2 & dual specificity phosphatase 2 \\
\hline 5 & 13671 & 8073960 & PIM3 & pim-3 oncogene \\
\hline 5 & 14495 & 8084880 & HES1 & hairy and enhancer of split 1, (Drosophila) \\
\hline 5 & 14598 & 8086330 & CSRNP1 & cysteine-serine-rich nuclear protein 1 \\
\hline 5 & 15667 & 8101304 & RASGEF1B & RasGEF domain family, member $1 \mathrm{~B}$ \\
\hline 5 & 16808 & 8116983 & CD83 & CD83 molecule \\
\hline 5 & 16819 & 8117128 & $\mathrm{E} 2 \mathrm{~F} 3$ & E2F transcription factor 3 \\
\hline 5 & 17039 & 8119712 & SRF & serum response factor (c-fos serum response elemen \\
\hline 5 & 17226 & 8122265 & TNFAIP3 & tumor necrosis factor, alpha-induced protein 3 \\
\hline 5 & 19535 & 8152215 & KLF10 & Kruppel-like factor 10 \\
\hline 5 & 20194 & 8160912 & C9orf131 & chromosome 9 open reading frame 131 \\
\hline 5 & 21196 & 8173437 & CXorf65 & chromosome $X$ open reading frame 65 \\
\hline 6 & 19176 & 8148059 & DEPDC6 & DEP domain containing 6 \\
\hline 6 & 405 & 7901788 & NFIA & nuclear factor I/A \\
\hline 6 & 533 & 7903592 & KIAA1324 & KIAA1324 \\
\hline 6 & 963 & 7909188 & IKBKE & inhibitor of kappa light polypeptide gene enhancer \\
\hline 6 & 1302 & 7913644 & E2F2 & E2F transcription factor 2 \\
\hline 6 & 1307 & 7913705 & CNR2 & cannabinoid receptor 2 (macrophage) \\
\hline 6 & 1816 & 7920552 & KCNN3 & potassium intermediate/small conductance calcium-a \\
\hline 6 & 1867 & 7921319 & FCRL1 & Fc receptor-like 1 \\
\hline 6 & 1945 & 7922219 & SELL & selectin L \\
\hline 6 & 5046 & 7961540 & RERG & RAS-like, estrogen-regulated, growth inhibitor \\
\hline 6 & 6464 & 7981183 & TCL1A & T-cell leukemia/lymphoma $1 \mathrm{~A}$ \\
\hline 6 & 7058 & 7989146 & MNS1 & meiosis-specific nuclear structural 1 \\
\hline 6 & 8905 & 8013319 & GRAP & GRB2-related adaptor protein \\
\hline 6 & 9993 & 8026787 & FAM129C & family with sequence similarity 129 , member $C$ \\
\hline 6 & 12385 & 8057377 & CCDC141 & coiled-coil domain containing 141 \\
\hline 6 & 12678 & 8061668 & $\mathrm{HCK}$ & hemopoietic cell kinase \\
\hline 6 & 12980 & 8065569 & BCL2L1 & BCL2-like 1 \\
\hline 6 & 13106 & 8067185 & BMP7 & bone morphogenetic protein 7 \\
\hline 6 & 13492 & 8071671 & GNAZ & guanine nucleotide binding protein (G protein), al \\
\hline 6 & 14419 & 8083749 & PPM1L & protein phosphatase, $\mathrm{Mg} 2+/ \mathrm{Mn} 2+$ dependent, $1 \mathrm{~L}$ \\
\hline 6 & 14487 & 8084766 & TP63 & tumor protein p63 \\
\hline 6 & 14576 & 8085984 & OSBPL10 & oxysterol binding protein-like 10 \\
\hline 6 & 16081 & 8107307 & CAMK4 & calcium/calmodulin-dependent protein kinase IV \\
\hline 6 & 16139 & 8108080 & PHF15 & PHD finger protein 15 \\
\hline 6 & 16542 & 8113504 & C5orf13 & chromosome 5 open reading frame 13 \\
\hline 6 & 17209 & 8122071 & ENPP3 & ectonucleotide pyrophosphatase/phosphodiesterase 3 \\
\hline
\end{tabular}




\begin{tabular}{|c|c|c|c|c|}
\hline 6 & 17721 & 8128818 & WASF1 & WAS protein family, member 1 \\
\hline 6 & 19841 & 8156321 & SYK & spleen tyrosine kinase \\
\hline 6 & 21437 & 8176174 & MPP1 & membrane protein, palmitoylated $1,55 \mathrm{kDa}$ \\
\hline 6 & 21884 & 8180299 & PSIP1 & PC4 and SFRS1 interacting protein 1 \\
\hline 7 & 17196 & 8121850 & HEY2 & hairy/enhancer-of-split related with YRPW motif 2 \\
\hline 7 & 661 & 7905299 & PRUNE & prune homolog (Drosophila) \\
\hline 7 & 1633 & 7918300 & PSRC1 & proline/serine-rich coiled-coil 1 \\
\hline 7 & 1646 & 7918457 & KCNA3 & potassium voltage-gated channel, shaker-related su \\
\hline 7 & 1973 & 7922474 & KIAA0040 & KIAA0040 \\
\hline 7 & 2529 & 7930139 & TRIM8 & tripartite motif-containing 8 \\
\hline 7 & 3627 & 7944152 & IL10RA & interleukin 10 receptor, alpha \\
\hline 7 & 4536 & 7954717 & $\mathrm{BICD} 1$ & bicaudal D homolog 1 (Drosophila) \\
\hline 7 & 5723 & 7971486 & C13orf18 & chromosome 13 open reading frame 18 \\
\hline 7 & 6012 & 7975361 & KIAA0247 & KIAA0247 \\
\hline 7 & 6968 & 7987385 & MEIS2 & Meis homeobox 2 \\
\hline 7 & 7736 & 7998336 & NARFL & nuclear prelamin A recognition factor-like \\
\hline 7 & 8540 & 8008297 & XYLT2 & xylosyltransferase II \\
\hline 7 & 8947 & 8013804 & DHRS13 & dehydrogenase/reductase (SDR family) member 13 \\
\hline 7 & 9001 & 8014551 & SYNRG & synergin, gamma \\
\hline 7 & 9103 & 8015655 & FAM134C & family with sequence similarity 134 , member $C$ \\
\hline 7 & 11798 & 8048489 & ANKZF1 & ankyrin repeat and zinc finger domain containing 1 \\
\hline 7 & 12072 & 8052669 & SERTAD2 & SERTA domain containing 2 \\
\hline 7 & 12622 & 8060977 & C20orf94 & chromosome 20 open reading frame 94 \\
\hline 7 & 14725 & 8088180 & WNT5A & wingless-type MMTV integration site family, member \\
\hline 7 & 15296 & 8096070 & BMP3 & bone morphogenetic protein 3 \\
\hline 7 & 15382 & 8097417 & PHF17 & PHD finger protein 17 \\
\hline 7 & 15454 & 8098423 & NEIL3 & nei endonuclease VIII-like 3 (E. coli) \\
\hline 7 & 15806 & 8103226 & TMEM154 & transmembrane protein 154 \\
\hline 7 & 15931 & 8104901 & IL7R & interleukin 7 receptor \\
\hline 7 & 16155 & 8108301 & KIF20A & kinesin family member $20 \mathrm{~A}$ \\
\hline 7 & 16602 & 8114365 & BRD8 & bromodomain containing 8 \\
\hline 7 & 16817 & 8117106 & RNF144B & ring finger protein $144 \mathrm{~B}$ \\
\hline 7 & 16821 & 8117165 & SOX4 & SRY (sex determining region Y)-box 4 \\
\hline 7 & 20457 & 8164252 & $\mathrm{SH} 2 \mathrm{D} 3 \mathrm{C}$ & $\mathrm{SH} 2$ domain containing $3 \mathrm{C}$ \\
\hline 9 & 11444 & 8043363 & NCRNA00152 & non-protein coding RNA 152 \\
\hline 9 & 540 & 7903703 & GNAI3 & guanine nucleotide binding protein (G protein), al \\
\hline 9 & 555 & 7903893 & CD53 & CD53 molecule \\
\hline 9 & 838 & 7907171 & BLZF1 & basic leucine zipper nuclear factor 1 \\
\hline 9 & 4515 & 7954436 & LRMP & lymphoid-restricted membrane protein \\
\hline 9 & 5501 & 7968297 & POMP & proteasome maturation protein \\
\hline 9 & 6023 & 7975545 & PSEN1 & presenilin 1 \\
\hline 9 & 6324 & 7979260 & GMFB & glia maturation factor, beta \\
\hline 9 & 6712 & 7983828 & TEX9 & testis expressed 9 \\
\hline 9 & 6954 & 7987172 & C15orf24 & chromosome 15 open reading frame 24 \\
\hline 9 & 7650 & 7997272 & GABARAPL2 & GABA(A) receptor-associated protein-like 2 \\
\hline
\end{tabular}




\begin{tabular}{|c|c|c|c|c|}
\hline 9 & 8087 & 8002878 & TMEM170A & transmembrane protein $170 \mathrm{~A}$ \\
\hline 9 & 8583 & 8008870 & TMEM49 & transmembrane protein 49 \\
\hline 9 & 13298 & 8069574 & C21orf91 & chromosome 21 open reading frame 91 \\
\hline 9 & 14333 & 8082607 & ATP2C1 & ATPase, Ca++ transporting, type $2 \mathrm{C}$, member 1 \\
\hline 9 & 14439 & 8084045 & MFN1 & mitofusin 1 \\
\hline 9 & 16532 & 8113358 & ST8SIA4 & ST8 alpha-N-acetyl-neuraminide alpha-2,8-sialyltra \\
\hline 9 & 16600 & 8114326 & FAM13B & family with sequence similarity 13 , member $B$ \\
\hline 9 & 17231 & 8122336 & C6orf115 & chromosome 6 open reading frame 115 \\
\hline 9 & 17345 & 8123961 & TBC1D7 & TBC1 domain family, member 7 \\
\hline 9 & 17484 & 8125463 & HLA-DQB2 & major histocompatibility complex, class II, DQ bet \\
\hline 9 & 17714 & 8128716 & CD164 & CD164 molecule, sialomucin \\
\hline 9 & 19076 & 8146550 & SDCBP & syndecan binding protein (syntenin) \\
\hline 9 & 20355 & 8162833 & ERP44 & endoplasmic reticulum protein 44 \\
\hline 9 & 20623 & 8166469 & SAT1 & spermidine/spermine N1-acetyltransferase 1 \\
\hline 9 & 21243 & 8174076 & GLA & galactosidase, alpha \\
\hline 9 & 21688 & 8178811 & NA & NA \\
\hline 9 & 21809 & 8180003 & NA & NA \\
\hline 9 & 21811 & 8180029 & HLA-DQB2 & major histocompatibility complex, class II, DQ bet \\
\hline 9 & 21856 & 8180268 & CYP51A1 & cytochrome $\mathrm{P} 450$, family 51 , subfamily A, polypepti \\
\hline 10 & 10233 & 8029693 & FOSB & FBJ murine osteosarcoma viral oncogene homolog B \\
\hline 10 & 357 & 7901140 & MAST2 & microtubule associated serine/threonine kinase 2 \\
\hline 10 & 535 & 7903632 & CELSR2 & cadherin, EGF LAG seven-pass G-type receptor 2 (fl \\
\hline 10 & 1511 & 7916403 & SSBP3 & single stranded DNA binding protein 3 \\
\hline 10 & 2234 & 7926037 & PFKFB3 & 6-phosphofructo-2-kinase/fructose-2,6-biphosphatas \\
\hline 10 & 3064 & 7937823 & KCNQ1 & potassium voltage-gated channel, KQT-like subfamil \\
\hline 10 & 6045 & 7975779 & FOS & FBJ murine osteosarcoma viral oncogene homolog \\
\hline 10 & 6175 & 7977344 & PACS2 & phosphofurin acidic cluster sorting protein 2 \\
\hline 10 & 6280 & 7978644 & NFKBIA & nuclear factor of kappa light polypeptide gene enh \\
\hline 10 & 6494 & 7981538 & JAG2 & jagged 2 \\
\hline 10 & 7111 & 7990033 & TLE3 & transducin-like enhancer of split 3 (E(sp1) homolo \\
\hline 10 & 8751 & 8011262 & MNT & MAX binding protein \\
\hline 10 & 8771 & 8011516 & ATP2A3 & ATPase, $\mathrm{Ca}++$ transporting, ubiquitous \\
\hline 10 & 9094 & 8015526 & KAT2A & $\mathrm{K}$ (lysine) acetyltransferase $2 \mathrm{~A}$ \\
\hline 10 & 9790 & 8024485 & GADD45B & growth arrest and DNA-damage-inducible, beta \\
\hline 10 & 9926 & 8026047 & JUNB & jun B proto-oncogene \\
\hline 10 & 10696 & 8034783 & LPHN1 & latrophilin 1 \\
\hline 10 & 11238 & 8040473 & RHOB & ras homolog gene family, member B \\
\hline 10 & 11322 & 8041763 & PRKCE & protein kinase $C$, epsilon \\
\hline 10 & 11474 & 8043657 & CNNM4 & cyclin M4 \\
\hline 10 & 12024 & 8051814 & ZFP36L2 & zinc finger protein $36, \mathrm{C} 3 \mathrm{H}$ type-like 2 \\
\hline 10 & 12269 & 8055130 & HS6ST1 & heparan sulfate 6-O-sulfotransferase 1 \\
\hline 10 & 16719 & 8115927 & RNF44 & ring finger protein 44 \\
\hline 10 & 17003 & 8119161 & PIM1 & pim-1 oncogene \\
\hline 10 & 17131 & 8121076 & PNRC1 & proline-rich nuclear receptor coactivator 1 \\
\hline 10 & 17886 & 8131091 & MAFK & v-maf musculoaponeurotic fibrosarcoma oncogene hom \\
\hline
\end{tabular}




\begin{tabular}{|c|c|c|c|c|}
\hline 10 & 19111 & 8147040 & ZBTB10 & zinc finger and BTB domain containing 10 \\
\hline 10 & 19342 & 8149720 & EGR3 & early growth response 3 \\
\hline 10 & 19848 & 8156452 & FAM120A & family with sequence similarity $120 \mathrm{~A}$ \\
\hline 10 & 20985 & 8170704 & $A B C D 1$ & ATP-binding cassette, sub-family D (ALD), member 1 \\
\hline 11 & 6845 & 7985493 & TM6SF1 & transmembrane 6 superfamily member 1 \\
\hline 11 & 1624 & 7918157 & VAV3 & vav 3 guanine nucleotide exchange factor \\
\hline 11 & 1660 & 7918634 & PHTF1 & putative homeodomain transcription factor 1 \\
\hline 11 & 2388 & 7928308 & DDIT4 & DNA-damage-inducible transcript 4 \\
\hline 11 & 2531 & 7930162 & C10orf26 & chromosome 10 open reading frame 26 \\
\hline 11 & 3218 & 7939465 & HSD17B12 & hydroxysteroid (17-beta) dehydrogenase 12 \\
\hline 11 & 3840 & 7946504 & TMEM41B & transmembrane protein 41B \\
\hline 11 & 4255 & 7951351 & PDGFD & platelet derived growth factor $\mathrm{D}$ \\
\hline 11 & 6422 & 7980547 & SEL1L & sel-1 suppressor of lin-12-like (C. elegans) \\
\hline 11 & 6474 & 7981290 & WARS & tryptophanyl-tRNA synthetase \\
\hline 11 & 7051 & 7989037 & CCPG1 & cell cycle progression 1 \\
\hline 11 & 7362 & 7993167 & ATF7IP2 & activating transcription factor 7 interacting prot \\
\hline 11 & 8563 & 8008646 & SCPEP1 & serine carboxypeptidase 1 \\
\hline 11 & 9189 & 8016578 & SLC35B1 & solute carrier family 35 , member B1 \\
\hline 11 & 9560 & 8021453 & SEC11C & SEC11 homolog C (S. cerevisiae) \\
\hline 11 & 12435 & 8058295 & ALS2 & amyotrophic lateral sclerosis 2 (juvenile) \\
\hline 11 & 13293 & 8069532 & HSPA13 & heat shock protein $70 \mathrm{kDa}$ family, member 13 \\
\hline 11 & 13346 & 8070083 & TMEM50B & transmembrane protein $50 \mathrm{~B}$ \\
\hline 11 & 13604 & 8073088 & APOBEC3G & apolipoprotein B mRNA editing enzyme, catalytic po \\
\hline 11 & 13688 & 8074204 & XKR3 & XK, Kell blood group complex subunit-related famil \\
\hline 11 & 14339 & 8082745 & CCRL1 & chemokine (C-C motif) receptor-like 1 \\
\hline 11 & 14893 & 8090737 & NPHP3 & nephronophthisis 3 (adolescent) \\
\hline 11 & 15565 & 8099897 & UGDH & UDP-glucose 6-dehydrogenase \\
\hline 11 & 15853 & 8103834 & AGA & aspartylglucosaminidase \\
\hline 11 & 15924 & 8104760 & TARS & threonyl-tRNA synthetase \\
\hline 11 & 17787 & 8129861 & IFNGR1 & interferon gamma receptor 1 \\
\hline 11 & 18115 & 8134091 & CLDN12 & claudin 12 \\
\hline 11 & 18474 & 8138789 & JAZF1 & JAZF zinc finger 1 \\
\hline 11 & 19697 & 8154531 & DENND4C & DENN/MADD domain containing $4 C$ \\
\hline 11 & 20881 & 8169541 & DOCK11 & dedicator of cytokinesis 11 \\
\hline 12 & 1897 & 7921625 & SLAMF6 & SLAM family member 6 \\
\hline 12 & 903 & 7908376 & RGS18 & regulator of G-protein signaling 18 \\
\hline 12 & 964 & 7909214 & RASSF5 & Ras association (RalGDS/AF-6) domain family member \\
\hline 12 & 2384 & 7928208 & SLC29A3 & solute carrier family 29 (nucleoside transporters) \\
\hline 12 & 3120 & 7938183 & ZNF215 & zinc finger protein 215 \\
\hline 12 & 3698 & 7945132 & FLI1 & Friend leukemia virus integration 1 \\
\hline 12 & 3762 & 7945875 & FAM86C & family with sequence similarity 86 , member $C$ \\
\hline 12 & 4451 & 7953651 & PEX5 & peroxisomal biogenesis factor 5 \\
\hline 12 & 4807 & 7958439 & USP30 & ubiquitin specific peptidase 30 \\
\hline 12 & 4873 & 7959354 & $\mathrm{BCL7A}$ & B-cell CLL/lymphoma 7A \\
\hline 12 & 5277 & 7964852 & BEST3 & bestrophin 3 \\
\hline
\end{tabular}




\begin{tabular}{|c|c|c|c|c|}
\hline 12 & 6046 & 7975787 & JDP2 & Jun dimerization protein 2 \\
\hline 12 & 6055 & 7975926 & KIAA1737 & KIAA1737 \\
\hline 12 & 6224 & 7977868 & C14orf93 & chromosome 14 open reading frame 93 \\
\hline 12 & 7059 & 7989159 & ZNF280D & zinc finger protein $280 \mathrm{D}$ \\
\hline 12 & 8674 & 8010139 & SEC14L1 & SEC14-like 1 (S. cerevisiae) \\
\hline 12 & 9654 & 8022803 & FAM59A & family with sequence similarity 59 , member $A$ \\
\hline 12 & 10907 & 8037186 & LIPE & lipase, hormone-sensitive \\
\hline 12 & 12455 & 8058570 & C2orf67 & chromosome 2 open reading frame 67 \\
\hline 12 & 14123 & 8079598 & ZNF589 & zinc finger protein 589 \\
\hline 12 & 15401 & 8097657 & SMAD1 & SMAD family member 1 \\
\hline 12 & 15518 & 8099200 & JAKMIP1 & janus kinase and microtubule interacting protein 1 \\
\hline 12 & 16165 & 8108447 & CXXC5 & CXXC finger 5 \\
\hline 12 & 16850 & 8117435 & BTN3A2 & butyrophilin, subfamily 3 , member $A 2$ \\
\hline 12 & 16854 & 8117476 & BTN3A3 & butyrophilin, subfamily 3 , member $A 3$ \\
\hline 12 & 17528 & 8126018 & STK38 & serine/threonine kinase 38 \\
\hline 12 & 18011 & 8132819 & IKZF1 & IKAROS family zinc finger 1 (Ikaros) \\
\hline 12 & 19193 & 8148317 & MYC & v-myc myelocytomatosis viral oncogene homolog (avi \\
\hline 12 & 20566 & 8165711 & PLCXD1 & phosphatidylinositol-specific phospholipase $C, X d$ \\
\hline 12 & 21450 & 8176286 & PLCXD1 & phosphatidylinositol-specific phospholipase $\mathrm{C}, \mathrm{X} \mathrm{d}$ \\
\hline 13 & 16874 & 8117630 & ZNF165 & zinc finger protein 165 \\
\hline 13 & 53 & 7897322 & PHF13 & PHD finger protein 13 \\
\hline 13 & 306 & 7900438 & ZNF643 & zinc finger protein 643 \\
\hline 13 & 869 & 7907690 & TOR3A & torsin family 3, member $A$ \\
\hline 13 & 1899 & 7921652 & SLAMF1 & signaling lymphocytic activation molecule family $\mathrm{m}$ \\
\hline 13 & 2654 & 7931914 & IL2RA & interleukin 2 receptor, alpha \\
\hline 13 & 3247 & 7939839 & PTPRJ & protein tyrosine phosphatase, receptor type, J \\
\hline 13 & 3306 & 7940118 & ZFP91-CNTF & ZFP91-CNTF readthrough transcript \\
\hline 13 & 4625 & 7955943 & PDE1B & phosphodiesterase 1B, calmodulin-dependent \\
\hline 13 & 7053 & 7989069 & PYGO1 & pygopus homolog 1 (Drosophila) \\
\hline 13 & 7145 & 7990555 & NRG4 & neuregulin 4 \\
\hline 13 & 7417 & 7994058 & SCNN1G & sodium channel, nonvoltage-gated 1 , gamma \\
\hline 13 & 8147 & 8003611 & FAM57A & family with sequence similarity 57 , member $A$ \\
\hline 13 & 8977 & 8014233 & SLFN11 & schlafen family member 11 \\
\hline 13 & 9135 & 8016088 & CCDC43 & coiled-coil domain containing 43 \\
\hline 13 & 9269 & 8017711 & GNA13 & guanine nucleotide binding protein (G protein), al \\
\hline 13 & 9951 & 8026350 & CLEC17A & C-type lectin domain family 17 , member $A$ \\
\hline 13 & 10765 & 8035694 & PBX4 & pre-B-cell leukemia homeobox 4 \\
\hline 13 & 10766 & 8035703 & LPAR2 & lysophosphatidic acid receptor 2 \\
\hline 13 & 11708 & 8047086 & NAB1 & NGFI-A binding protein 1 (EGR1 binding protein 1) \\
\hline 13 & 12581 & 8060418 & SIRPA & signal-regulatory protein alpha \\
\hline 13 & 15333 & 8096635 & NFKB1 & nuclear factor of kappa light polypeptide gene enh \\
\hline 13 & 15555 & 8099760 & ARAP2 & ArfGAP with RhoGAP domain, ankyrin repeat and PH d \\
\hline 13 & 16707 & 8115806 & UBTD2 & ubiquitin domain containing 2 \\
\hline 13 & 16929 & 8118137 & LTA & lymphotoxin alpha (TNF superfamily, member 1) \\
\hline 13 & 17232 & 8122343 & HECA & headcase homolog (Drosophila) \\
\hline
\end{tabular}




\begin{tabular}{|c|c|c|c|c|}
\hline 13 & 17259 & 8122756 & PLEKHG1 & pleckstrin homology domain containing, family G (w \\
\hline 13 & 18983 & 8145136 & PPP3CC & protein phosphatase 3 , catalytic subunit, gamma is \\
\hline 13 & 21600 & 8177976 & LTA & lymphotoxin alpha (TNF superfamily, member 1) \\
\hline 13 & 21736 & 8179258 & LTA & lymphotoxin alpha (TNF superfamily, member 1) \\
\hline 14 & 453 & 7902512 & DNAJB4 & DnaJ (Hsp40) homolog, subfamily B, member 4 \\
\hline 14 & 197 & 7899087 & PDIK1L & PDLIM1 interacting kinase 1 like \\
\hline 14 & 612 & 7904755 & PEX11B & peroxisomal biogenesis factor 11 beta \\
\hline 14 & 1322 & 7913883 & PAFAH2 & platelet-activating factor acetylhydrolase $2,40 \mathrm{kD}$ \\
\hline 14 & 1617 & 7918008 & DBT & dihydrolipoamide branched chain transacylase E2 \\
\hline 14 & 1745 & 7919780 & GOLPH3L & golgi phosphoprotein 3-like \\
\hline 14 & 1991 & 7922707 & RNASEL & ribonuclease $L(2$ ',5'-oligoisoadenylate synthetase \\
\hline 14 & 2064 & 7923812 & RAB7L1 & RAB7, member RAS oncogene family-like 1 \\
\hline 14 & 2720 & 7933092 & ZNF248 & zinc finger protein 248 \\
\hline 14 & 2770 & 7933638 & FLJ31958 & hypothetical LOC143153 \\
\hline 14 & 2860 & 7934945 & PANK1 & pantothenate kinase 1 \\
\hline 14 & 4463 & 7953765 & RIMKLB & ribosomal modification protein rimk-like family me \\
\hline 14 & 5288 & 7965060 & BBS10 & Bardet-Biedl syndrome 10 \\
\hline 14 & 5577 & 7969374 & C13orf34 & chromosome 13 open reading frame 34 \\
\hline 14 & 6768 & 7984626 & SENP8 & SUMO/sentrin specific peptidase family member 8 \\
\hline 14 & 7373 & 7993298 & ERCC4 & excision repair cross-complementing rodent repair \\
\hline 14 & 10902 & 8037144 & DEDD2 & death effector domain containing 2 \\
\hline 14 & 11666 & 8046502 & SCRN3 & secernin 3 \\
\hline 14 & 12185 & 8053975 & LMAN2L & lectin, mannose-binding 2-like \\
\hline 14 & 12454 & 8058552 & IDH1 & isocitrate dehydrogenase 1 (NADP+), soluble \\
\hline 14 & 12927 & 8064967 & MKKS & McKusick-Kaufman syndrome \\
\hline 14 & 15551 & 8099696 & SEPSECS & Sep (O-phosphoserine) tRNA:Sec (selenocysteine) tR \\
\hline 14 & 15741 & 8102362 & TIFA & TRAF-interacting protein with forkhead-associated \\
\hline 14 & 16759 & 8116494 & ZFP62 & zinc finger protein 62 homolog (mouse) \\
\hline 14 & 16881 & 8117685 & ZKSCAN3 & zinc finger with KRAB and SCAN domains 3 \\
\hline 14 & 16991 & 8119000 & MAPK14 & mitogen-activated protein kinase 14 \\
\hline 14 & 17767 & 8129590 & STX7 & syntaxin 7 \\
\hline 14 & 20547 & 8165486 & TMEM203 & transmembrane protein 203 \\
\hline 14 & 20561 & 8165642 & TMEM203 & transmembrane protein 203 \\
\hline 14 & 21087 & 8171896 & CXorf21 & chromosome $\mathrm{X}$ open reading frame 21 \\
\hline 17 & 20331 & 8162533 & PTCH1 & patched 1 \\
\hline 17 & 265 & 7899870 & ZNF362 & zinc finger protein 362 \\
\hline 17 & 2924 & 7935968 & LDB1 & LIM domain binding 1 \\
\hline 17 & 3452 & 7941797 & ADRBK1 & adrenergic, beta, receptor kinase 1 \\
\hline 17 & 3466 & 7942007 & LRP5 & low density lipoprotein receptor-related protein 5 \\
\hline 17 & 4073 & 7949206 & MEN1 & multiple endocrine neoplasia I \\
\hline 17 & 4075 & 7949264 & EHD1 & EH-domain containing 1 \\
\hline 17 & 5108 & 7962659 & HDAC7 & histone deacetylase 7 \\
\hline 17 & 6899 & 7986359 & IGF1R & insulin-like growth factor 1 receptor \\
\hline 17 & 7421 & 7994109 & PLK1 & polo-like kinase 1 \\
\hline 17 & 7680 & 7997680 & KIAA0182 & KIAA0182 \\
\hline
\end{tabular}




\begin{tabular}{|c|c|c|c|c|}
\hline 17 & 8452 & 8007302 & TUBG1 & tubulin, gamma 1 \\
\hline 17 & 9329 & 8018708 & UBE2O & ubiquitin-conjugating enzyme E2O \\
\hline 17 & 9330 & 8018731 & RHBDF2 & rhomboid 5 homolog 2 (Drosophila) \\
\hline 17 & 9439 & 8019778 & PCYT2 & phosphate cytidylyltransferase 2 , ethanolamine \\
\hline 17 & 9973 & 8026533 & $\mathrm{HSH} 2 \mathrm{D}$ & hematopoietic SH2 domain containing \\
\hline 17 & 10166 & 8028930 & RAB4B & RAB4B, member RAS oncogene family \\
\hline 17 & 10582 & 8033362 & INSR & insulin receptor \\
\hline 17 & 11854 & 8049317 & DGKD & diacylglycerol kinase, delta $130 \mathrm{kDa}$ \\
\hline 17 & 13486 & 8071566 & PPIL2 & peptidylprolyl isomerase (cyclophilin)-like 2 \\
\hline 17 & 13800 & 8075430 & MORC2 & MORC family CW-type zinc finger 2 \\
\hline 17 & 14078 & 8079037 & TRAK1 & trafficking protein, kinesin binding 1 \\
\hline 17 & 14177 & 8080487 & PRKCD & protein kinase $\mathrm{C}$, delta \\
\hline 17 & 15732 & 8102232 & LEF1 & lymphoid enhancer-binding factor 1 \\
\hline 17 & 17465 & 8125172 & EHMT2 & euchromatic histone-lysine $\mathrm{N}$-methyltransferase 2 \\
\hline 17 & 18184 & 8135149 & $\mathrm{SH} 2 \mathrm{~B} 2$ & SH2B adaptor protein 2 \\
\hline 17 & 19665 & 8154059 & SMARCA2 & SWI/SNF related, matrix associated, actin dependen \\
\hline 17 & 20529 & 8165217 & NOTCH1 & notch 1 \\
\hline 17 & 21198 & 8173457 & ZMYM3 & zinc finger, MYM-type 3 \\
\hline 17 & 21800 & 8179884 & EHMT2 & euchromatic histone-lysine $\mathrm{N}$-methyltransferase 2 \\
\hline 19 & 4234 & 7951077 & SESN3 & sestrin 3 \\
\hline 19 & 525 & 7903507 & FAM102B & family with sequence similarity 102 , member $B$ \\
\hline 19 & 889 & 7908041 & LAMC1 & laminin, gamma 1 (formerly LAMB2) \\
\hline 19 & 920 & 7908543 & NEK7 & NIMA (never in mitosis gene a)-related kinase 7 \\
\hline 19 & 1629 & 7918255 & CLCC1 & chloride channel CLIC-like 1 \\
\hline 19 & 2217 & 7925792 & ZMYND11 & zinc finger, MYND domain containing 11 \\
\hline 19 & 2465 & 7929282 & HHEX & hematopoietically expressed homeobox \\
\hline 19 & 2674 & 7932227 & NMT2 & $\mathrm{N}$-myristoyltransferase 2 \\
\hline 19 & 2689 & 7932512 & DNAJC1 & DnaJ (Hsp40) homolog, subfamily C, member 1 \\
\hline 19 & 2901 & 7935627 & GOT1 & glutamic-oxaloacetic transaminase 1 , soluble (aspa \\
\hline 19 & 5095 & 7962455 & NELL2 & NEL-like 2 (chicken) \\
\hline 19 & 7055 & 7989094 & NEDD4 & neural precursor cell expressed, developmentally $d$ \\
\hline 19 & 8525 & 8008113 & CALCOCO2 & calcium binding and coiled-coil domain 2 \\
\hline 19 & 9621 & 8022356 & SPIRE1 & spire homolog 1 (Drosophila) \\
\hline 19 & 11271 & 8040927 & NRBP1 & nuclear receptor binding protein 1 \\
\hline 19 & 11833 & 8049044 & ARMC9 & armadillo repeat containing 9 \\
\hline 19 & 13074 & 8066716 & ELMO2 & engulfment and cell motility 2 \\
\hline 19 & 14105 & 8079334 & LIMD1 & LIM domains containing 1 \\
\hline 19 & 14422 & 8083779 & SERPINI1 & serpin peptidase inhibitor, clade I (neuroserpin), \\
\hline 19 & 14427 & 8083839 & GPR160 & G protein-coupled receptor 160 \\
\hline 19 & 16557 & 8113691 & DTWD2 & DTW domain containing 2 \\
\hline 19 & 16945 & 8118310 & HSPA1A & heat shock $70 \mathrm{kDa}$ protein $1 \mathrm{~A}$ \\
\hline 19 & 17352 & 8124040 & ATXN1 & ataxin 1 \\
\hline 19 & 17729 & 8128956 & FYN & FYN oncogene related to SRC, FGR, YES \\
\hline 19 & 18711 & 8141688 & PLOD3 & procollagen-lysine, 2-oxoglutarate 5-dioxygenase 3 \\
\hline 19 & 18883 & 8143961 & PRKAG2 & protein kinase, AMP-activated, gamma 2 non-catalyt \\
\hline
\end{tabular}




\begin{tabular}{|l|l|l|l|l|}
\hline 19 & 19050 & 8146243 & HOOK3 & hook homolog 3 (Drosophila) \\
\hline $\mathbf{1 9}$ & 19356 & 8149877 & PNMA2 & paraneoplastic antigen MA2 \\
\hline $\mathbf{1 9}$ & 19675 & 8154178 & JAK2 & Janus kinase 2 \\
\hline $\mathbf{1 9}$ & $\mathbf{2 1 7 4 4}$ & 8179322 & HSPA1A & heat shock 70kDa protein 1A \\
\hline
\end{tabular}




\title{
Curriculum vitae
}

\author{
Particulars \\ \begin{tabular}{c|l} 
Name & Elisabeth Hand \\
Date of birth & $14-06-1985$ in Berlin - Pankow
\end{tabular} \\ Nationality \\ German \\ Marital status \\ Married \\ Tertiary and \\ secondary \\ education \\ Higher education \\ 2009 Ph.D. candidate in Molecular Medicine, Department of \\ Haematology and Oncology under the supervision of Prof. Dr. \\ Dieter Kube, University Medicine Göttingen, \\ 2007 - 2009 Molecular Medicine, M.Sc., Georg-August \\ University, Göttingen \\ Master thesis: The influence of Ephrin B3 and Semaphorin 3A \\ on CNS remyelination, Max Planck Institute for Experimental \\ Medicine, Department for Neurogenetics, Göttingen, \\ 2004 - 2007 Molecular Life Science, B.Sc., University of Lübeck \\ Bachelor thesis: Optimization of expression and purification of \\ a Maltose-Binding-Protein-Falcipain2-fusionprotein, Institute \\ for Biochemistry, University of Lübeck \\ Secondary school \\ 2002 - 2004 Abitur, German School Istanbul, Turkey, \\ 2001 - 2002 Exchange student, Collège Notre-Dame-de- \\ I'Assomption, Nicolet, Québec, Canada, \\ 1997 - 2001 Gerhart-Hauptmann-Gymnasium, Berlin \\ Other skills and \\ experiences \\ Languages \\ German - native speaker \\ English - fluent \\ French - fluent
}




\section{Publications}

Global gene expression changes of in vitro stimulated human transformed germinal centre $B$ cells as surrogate for oncogenic pathway activation in individual aggressive B cell lymphomas.

A. Schrader, K. Meyer, F. von Bonin, M. Vockerodt, N. Walther, E. Hand, A. Ulrich, K. Matulewicz, D. Lenze, M. Hummel, A. Kieser, M. Engelke, L. Trümper, D. Kube

Cell Communication and Signaling: CCS 2012 Dec 20. doi: 10.1186/1478-811X-10-43 PMID: 23253402

Inhibition of CNS remyelination by the presence of semaphorin 3A.

Y.A. Syed, E. Hand, W. Möbius, C. Zhao, M. Hofer, K.A. Nave, M.R. Kotter

The Journal of Neuroscience 2011 Mar 9; doi: 10.1523/JNEUROSCI.4930-10.2011 PMID: 21389227

Multikinase inhibitor sorafenib exerts cytocidal efficacy against Non-Hodgkin lymphomas associated with inhibition of MAPK14 and AKT phosphorylation.

B. Chapuy, N. Schuelper, M. Panse, A. Dohm, E. Hand, R. Schroers, L. Truemper, G.G. Wulf

British Journal of Haematology 2011 Feb; doi: 10.1111/j.1365-2141.2010.08526.x PMID: 21689083 


\section{Danksagung}

Zunächst möchte ich Prof. Dr. Dieter Kube danken, der diese Arbeit ermöglicht hat. Er hat mir in den letzten 4 Jahren stets mit wertvollen Ratschlägen und Hinweisen zur Seite gestanden und mit seinen Ideen und seiner Diskussionsbereitschaft entscheidend diese Arbeit geprägt. Außerdem bin ich ihm sehr dankbar, dass er immer vollstes Verständnis für meine familiäre Situation hatte. Ich bin mir dessen bewußt, dass das keine Selbstverständlichkeit ist und danke ihm für sein Vertrauen, sein Entgegenkommen und seine moralische Unterstützung.

Ich danke auch den Mitgliedern meines Thesis Committees: Prof. Dr. Mika Simons für die durchgehende Unterstützung schon seit der Anfertigung meiner Masterarbeit, Prof. Dr. Steven Johnson für die immer wieder positiven Anmerkungen und Aufmunterungen, sowie Prof. Dr. Peter Burfeind für seine kurzfristige Einwilligung als neues drittes Mitglied im Thesis Committee mitzuwirken.

Großer Dank geht auch an den Verbund HaematoSys (gefördert durch das Bundesministerium für Bildung und Forschung (BMBF) und geleitet von Prof. Dr. Markus Löffler), in dessen Rahmen diese Arbeit durchgeführt wurde und dessen Verbundtreffen in Leipzig immer ein Erlebnis, sowohl wissenschaftlicher als auch kultureller Art waren. Besonderer Dank gilt an dieser Stelle auch den Verbundmitgliedern in Leipzig und Regensburg, die die statistische und bioinformatische Aufarbeitung der hier generierten Daten und Fragestellungen übernommen haben. Dr. Bernd Klaus und Prof. Dr. Korbinian Strimmer danke ich für die geduldigen Erklärungen zu den Netzwerkanalysen und die kurzweiligen Diskussionen in Leipzig. Prof. Dr. Jürgen Läuter danke ich für das beherzte „Drauf-Los-Rechnen“ bei den Zeitreihendaten und das stete Bemühen unsere Analysen voranzutreiben. Herzlicher Dank geht an Prof. Dr. Rainer Spang und seine Arbeitsgruppe computational diagnostics in Regensburg, ohne deren bioinformatische Expertise die vorliegenden Analysen nicht möglich gewesen wären, die aber auch immer ausreichend biologisches Verständnis gezeigt haben um eine anregende Diskussion zu ermöglichen. Besonderer Dank geht hierbei an Dr. Katharina Meyer und Martin Pirkl, die immer fleißig alle Berechnungen angefertigt haben, die ich angefordert habe und trotzdem nie müde wurden, mir ihre Vorgehensweisen zu erläutern.

Des Weiteren bedanke ich mich sehr bei Prof. Dr. Heidi Hahn und Dr. Anja Uhman aus dem Institut für Humangenetik für die ersten Einblicke ins Hedgehog-Signaling sowie die großzügige Bereitstellung aller Zelllinien, Plasmide, Assays und Primer, die die Bearbeitung des HedgehogKapitels überhaupt erst ermöglichten. 
Bei Prof. Dr. Arnd Kieser und seiner AG möchte ich mich für die Unterstützung bei dem Tak1Projekt und die großzügige Bereitstellung aller Informationen, Plasmide und Antikörper zu TNIK bedanken, auch wenn diese Studien nicht den Eingang in diese Arbeit geschafft haben.

Herzlicher Dank geht an die Kollegen aus der AG Kube (auch und besonders an die Ehemaligen) für die unglaublich tolle Arbeitsatmosphäre, die stete Unterstützung moralischer wie auch technischer Natur sowie die regelmäßige Versorgung mit Kuchen. Besonders erwähnen möchte ich an dieser Stelle Frederike von Bonin, Susanne Hengst und Neele Walther, die mir nicht nur technisch im Labor durch erstaunliche Fähigkeiten geholfen. Sie haben mir immer wieder Arbeit abgenommen, wenn mal wieder die KiTa angerufen hat und waren stets eine große moralische Unterstützung. An Neele geht mein Dank auch für die unzähligen Stunden der Kinderbetreuung. An dieser Stelle möchte ich mich bei allen fleißigen Korrekturleserinnen bedanken: Franziska Linke, Dr. Sonja Eberth, Juliane Lippert, Natalie Klytta, Dr. Christina Heemann, Dr. Alexandra Schrader und Dr. Julia Bode.

Mein ganz besonderer Dank gilt Alexandra, die mich zu Beginn meiner Arbeit unter ihre Fittiche genommen hat und mir nicht nur eine sehr gute Kollegin war, sondern auch eine super Freundin geworden ist. Ihre Arbeiten waren nicht nur die Grundlage für die vorliegende Studie, die Diskussionen im Büro und gemeinsamen Stunden in der Zellkultur haben auch wesentlich zur Weiterentwicklung des Projektes beigetragen. Ich danke ihr und Julia besonders herzlich für das Korrekturlesen dieser Arbeit auch zu späten Nachtstunden und die vielen, unbezahlbaren Momente im Büro und in der Freizeit. Es ist schön, dass aus Kolleginnen auch Freundinnen werden können! Den Mädels vom ehemaligen Ost-Büro danke ich für die schöne Zeit und die vielen Anregungen aus den Kaffee-Pausen.

Meinen Eltern danke ich für ihren Glauben an mich und dass sie mir immer geholfen haben, meine Ziele zu erreichen. Außerdem danke ich ihnen, genauso wie meinen Schwiegereltern, für die unsagbar große Hilfe in den letzten Wochen vor der Abgabe dieser Arbeit. Meinem Ehemann Thomas und meinem Sohn Johann danke ich von ganzem Herzen für die schöne Ablenkung in Familie nach der Arbeitszeit und das große Verständnis und die Unterstützung wenn „Mama arbeiten muss“. 